\title{
Use of 3D Seismic Azimuthal Iso-Frequency Volumes for the Detection and Characterization of High Porosity/Permeability Zones in Carbonate Reservoirs
}

\author{
Brian E. Toelle \\ West Virginia University
}

Follow this and additional works at: https://researchrepository.wvu.edu/etd

\author{
Recommended Citation \\ Toelle, Brian E., "Use of 3D Seismic Azimuthal Iso-Frequency Volumes for the Detection and \\ Characterization of High Porosity/Permeability Zones in Carbonate Reservoirs" (2012). Graduate Theses, \\ Dissertations, and Problem Reports. 222. \\ https://researchrepository.wvu.edu/etd/222
}

This Dissertation is protected by copyright and/or related rights. It has been brought to you by the The Research Repository @ WVU with permission from the rights-holder(s). You are free to use this Dissertation in any way that is permitted by the copyright and related rights legislation that applies to your use. For other uses you must obtain permission from the rights-holder(s) directly, unless additional rights are indicated by a Creative Commons license in the record and/ or on the work itself. This Dissertation has been accepted for inclusion in WVU Graduate Theses, Dissertations, and Problem Reports collection by an authorized administrator of The Research Repository @ WVU.

For more information, please contact researchrepository@mail.wvu.edu. 


\title{
Use of 3D Seismic Azimuthal Iso-Frequency Volumes for the Detection and Characterization of High Porosity/Permeability Zones in Carbonate Reservoirs
}

\author{
Brian E. Toelle \\ Thesis submitted to the \\ Eberly College of Arts and Sciences at West Virginia University \\ in partial fulfillment of the requirement for the degree of \\ Ph.D. in Geology
}

\section{Committee}

Dr. Tom Wilson, Ph.D., Dept. of Geology and Geography (Chair)

Dr. Jaime Toro, Ph.D., Dept. of Geology and Geography

Dr. Tim Carr, Ph.D., Dept. of Geology and Geography

Dr. Dengliang Gao, Ph.D., Dept. of Geology and Geography

Dr. Michael Grammer, Ph.D., Dept. of Geology, Oklahoma State University

Department of Geology and Geography

Morgantown, West Virginia

2012

Keywords: 3d seismic, Azimuthal Seismic, Spectral Decomposition, Iso-frequency volumes, reservoir characterization, carbonate reservoirs 


\section{ABSTRACT \\ Use of 3D Seismic Azimuthal Iso-Frequency Volumes for the Detection and Characterization of High Porosity/Permeability Zones in Carbonates}

Brian E. Toelle

Among the most important properties controlling the production from conventional oil and gas reservoirs is the distribution of porosity and permeability within the producing geologic formation. The geometry of the pore space within these reservoirs, and the permeability associated with this pore space geometry, impacts not only where production can occur and at what flow rates but can also have significant influence on many other rock properties. Zones of high matrix porosity can result in an isotropic response for certain reservoir properties whereas aligned porosity/permeability, such as open, natural fracture trends, have been shown to result in reservoirs being anisotropic in many properties.

The ability to identify zones within a subsurface reservoir where porosity/permeability is significantly higher and to characterize them according to their geometries would be of great significance when planning where new boreholes, particularly horizontal boreholes, should be drilled. The detection and characterization of these high porosity/permeability zones using their isotropic and anisotropic responses may be possible through the analysis of azimuthal (also referred to as azimuth-limited) 3D seismic volumes.

During this study the porosity/permeability systems of a carbonate, pinnacle reef within the northern Michigan Basin undergoing enhanced oil recovery were investigated using selected seismic attributes extracted from azimuthal 3D seismic volumes. Based on the response of these seismic attributes an interpretation of the geometry of the porosity/permeability system within the reef was made. This interpretation was supported by well data that had been obtained during the primary production phase of the field. Additionally, 4D seismic data, obtained as part of the $\mathrm{CO}_{2}$ based EOR project, supported reservoir simulation results that were based on the porosity/permeability interpretation. 


\section{ACKNOWLEDGEMENT}

I would first like to acknowledge and extend my grateful appreciation to all members of my committee. Dr. Jamie Toro, Dr. Timothy R. Carr and Dr. Dengliang Gao from the department here at West Virginia University, as well as Dr. G. Michael Grammer of Oklahoma State University, have all been quite supportive of this research and have offered many insightful comments on this research that have been extremely helpful.

Undoubtedly the greatest amount of guidance that I have received through this effort has been from Dr. Tom Wilson, my committee chairman. I wish to recognize his steadfast direction and leadership during my involvement with West Virginia University without which I am certain this effort would have taken significantly longer. I am grateful to him for his support and guidance throughout this undertaking.

I would also like to thank the United States Department of Energy for the initial funding of some of this research and Schlumberger for allowing me the opportunity to continue my education with in my chosen field.

But most importantly I have to thank my family and acknowledge their unwavering support during the many years that I have pursued this research. Both of my sons, Ryan and Patrick, have continually inspired me in this effort and have been extremely understanding when I have had to miss various family events in order to work on this degree. Additionally, and without a doubt, I would not have been able to complete this effort without the constant support of my wife, Carole. Without her assistance, in all aspects of our lives, I could not have even considered this endeavor. During our 33 years of marriage she has always been my greatest inspiration. 


\section{TABLE OF CONTENTS}

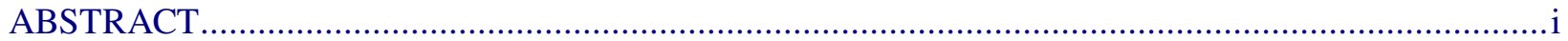

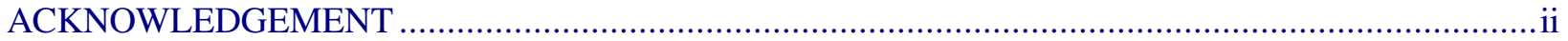

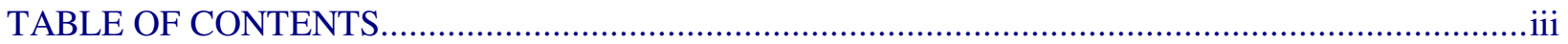

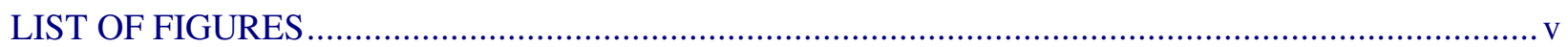

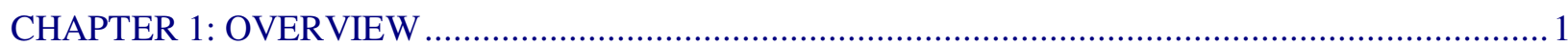

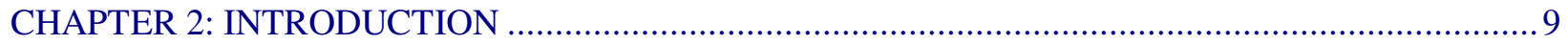

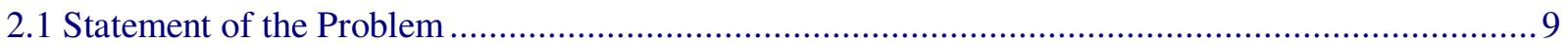

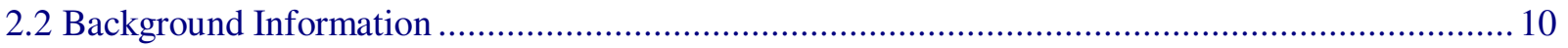

2.2.1 Previous Work - High Frequency Attenuation as an indicator of Porosity / Permeability ....... 10

2.2.2 Previous Work - Spectral Decomposition............................................................... 22

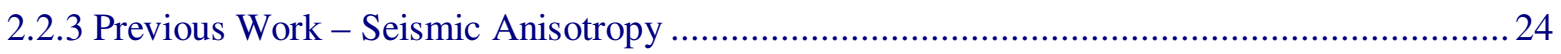

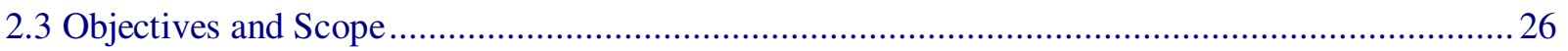

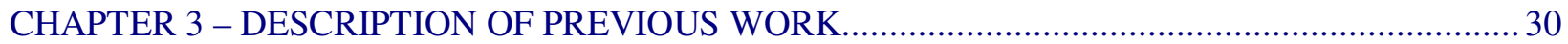

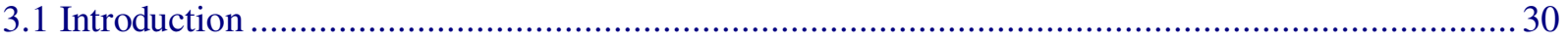

3.1.1 The Northern Silurian Reef Trend of the Michigan Basin .............................................. 30

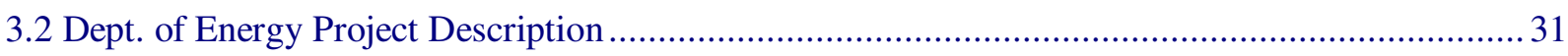

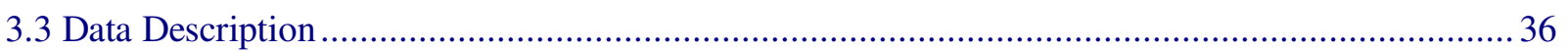

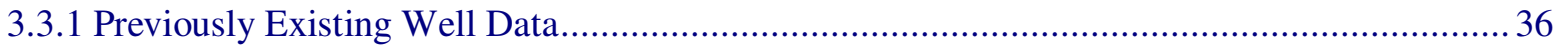

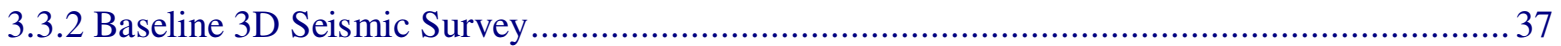

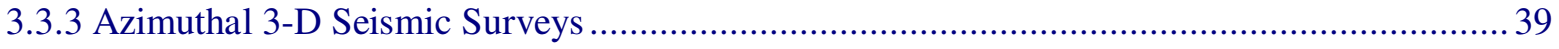

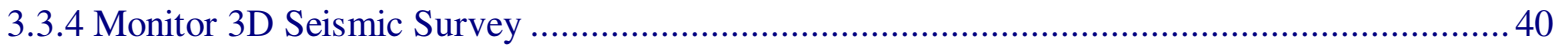

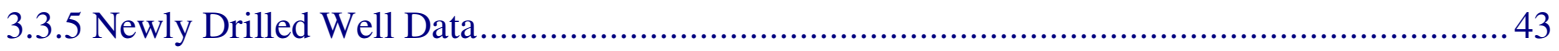

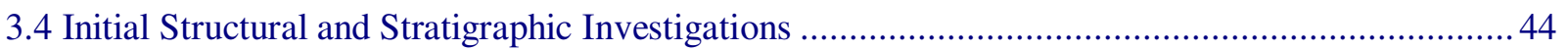

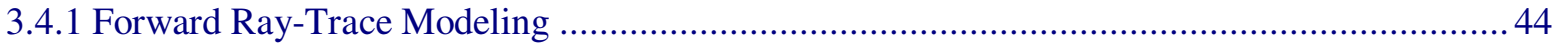

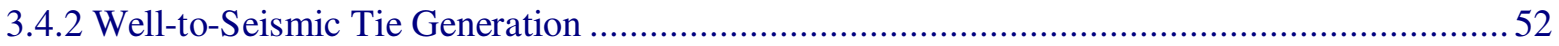

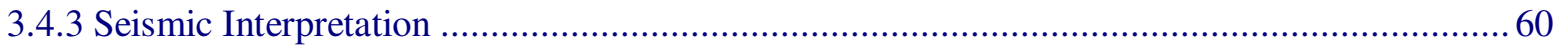

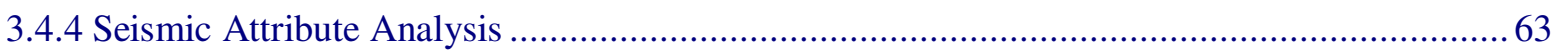

CHAPTER 4 - FREQUENCY ATTENUATION AND ITS RELATIONSHIP TO DRAINED, HIGH

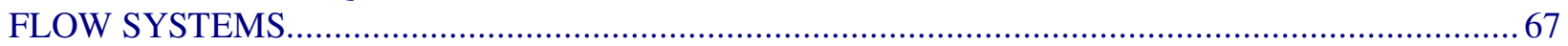

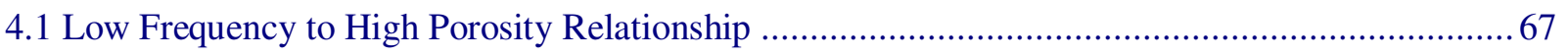

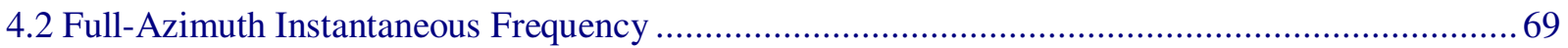

4.3 Porosity Distribution and Reservoir Characterization .............................................................. 74

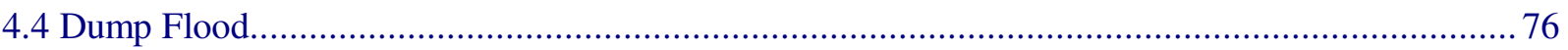

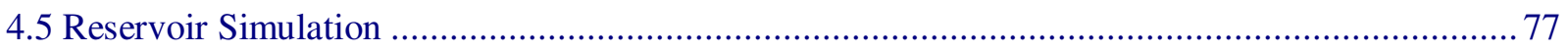

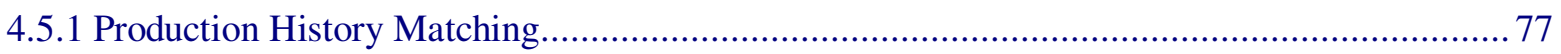




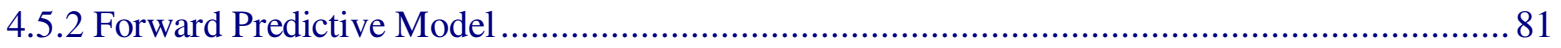

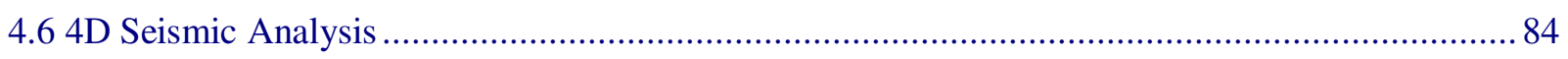

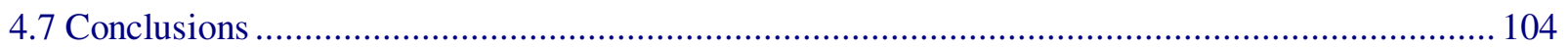

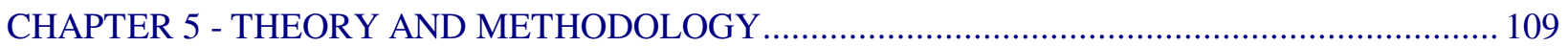

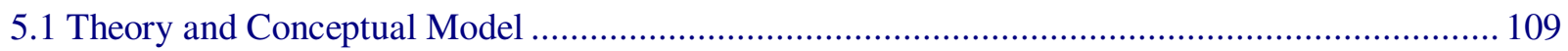

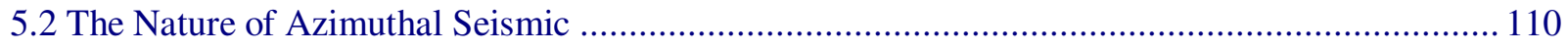

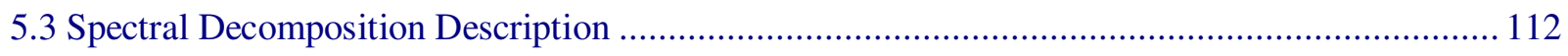

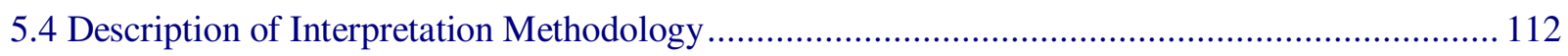

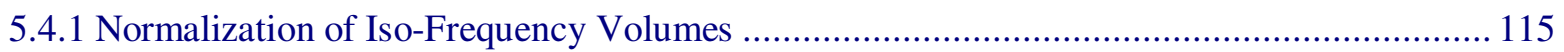

CHAPTER 6 - AZIMUTHAL ISO-FREQUENCY INTERPRETATION ….................................... 120

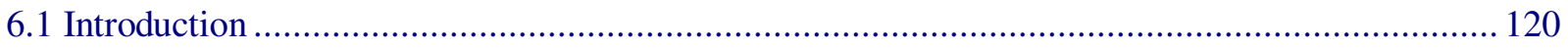

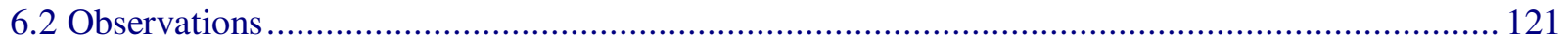

6.2.1 Comparison of All Azimuth Fraction (AAF) and Azimuthal Iso-Frequency Volumes........... 123

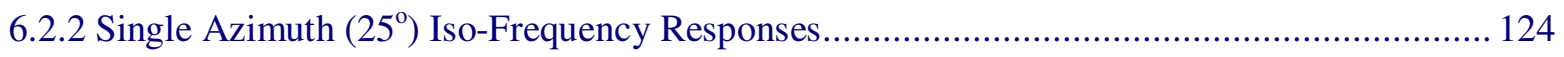

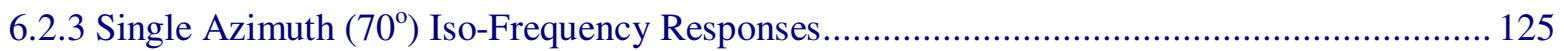

6.2.4 Iso-Frequency comparisons along the $115^{\circ}$ and $160^{\circ}$ Azimuths ....................................... 127

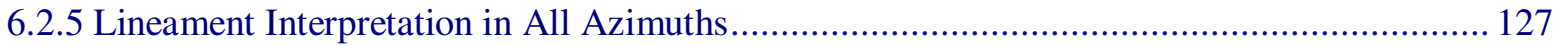

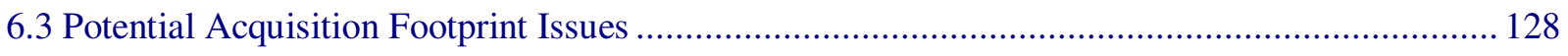

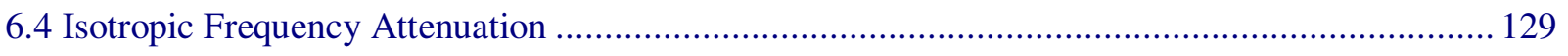

6.5 Isotropic Frequency Attenuation and Correlation with Previous Porosity Analyses ................... 132

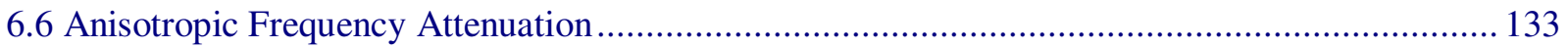

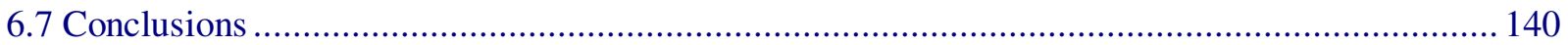

CHAPTER 7 - DRAINED, HIGH FLOW SYSTEM CHARACTERIZATION AND COMPARISON

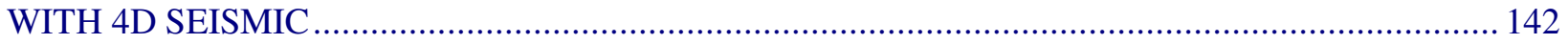

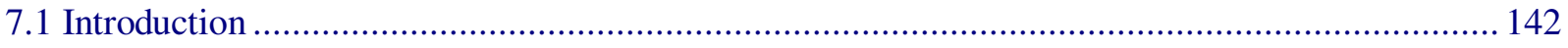

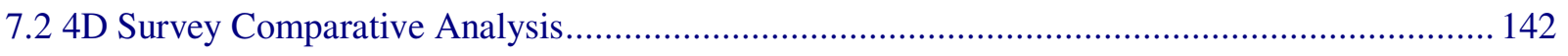

7.3 Iso-frequency Normalized Comparative Analysis ............................................................. 143

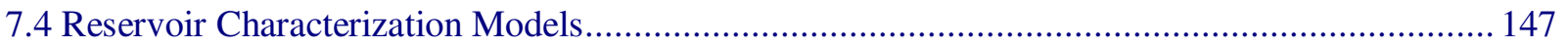

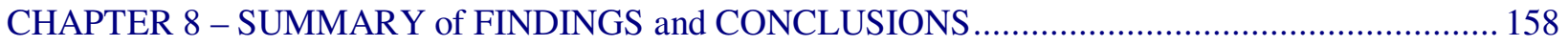

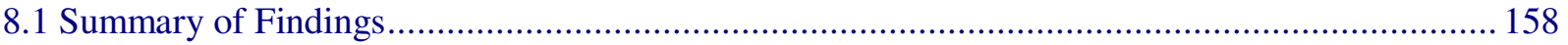

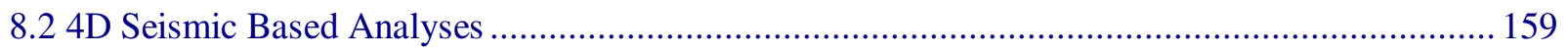

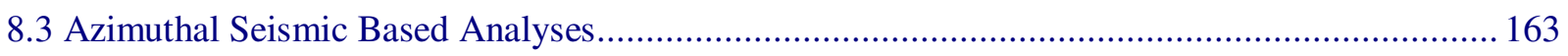

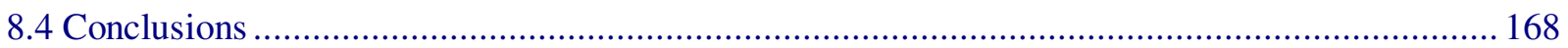

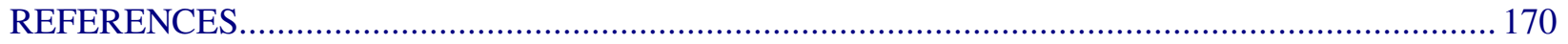




\section{LIST OF FIGURES}

Figure 1: MULLER'S FiguRE \#2 ILLUSTRATING THE COMPRESSIONAL AND EXTENSIONAL PHASES OF A WAVELET PASSAGE THROUGH A POROUS MEDIA.

FIGURE 2: AZIMUTHAL CHANGES IN VELOCITY AND ATTENUATION WITH RESPECT TO A SINGLE MESOSCOPIC FRACTURE SET FOR FREQUENCIES $20 \mathrm{~Hz}$ (BLACK), $45 \mathrm{~Hz}$ (bLUE), $60 \mathrm{~Hz}$ (RED) AND $90 \mathrm{~Hz}$ (GREEN). ModifIEd FROM Ali (2011) FOR AN ANGLE OF INCIDENCE OF $40^{\circ}$ WITH RESPECT TO THE FRACTURE SET.

\section{5}

FIGURE 3: ATTENUATION TRENDS AS A FUNCTION OF POROSITY TAKEN FROM BRADLEY AND FORT (1966). ............................... 11

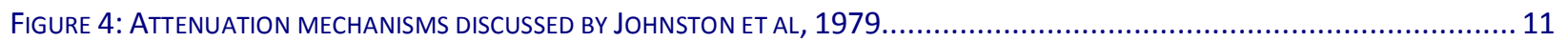

FIGURE 5: JOHNSTON ET AL, 1979 FIGURE \#14 SHOWING P-WAVE ATTENUATION COEFFICIENTS AT SURFACE PRESSURE AS A FUNCTION OF

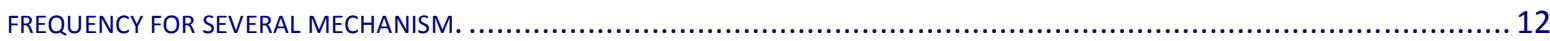

FIGURE 6: MAVKO'S FIGURE 4 SHOWING THE FREQUENCY DEPENDENCY OF $Q^{-1}$ FOR THE PARALLEL WALLED PORE IN COMPRESSION. ....... 13

FIGURE 7: WHITE'S FIGURE \#2 SHOWING THE RELATIONSHIP BETWEEN FREQUENCY, PHASE ANGLE AND VELOCITY. ......................... 13

FIGURE 8: ILLUSTRATIONS FROM WINKLER, 1983 SHOWING THAT ATTENUATION FOR DRY (DASHED) AND BRINE SATURATED (SOLID) FUSED GLASS BEADS (ON LEFT) AND ONE OF THE FORMATIONS TEST AT VARIOUS CONFINING PRESSURES................................... 14

FIGURE 9: SHEAR WAVE ATTENUATION VERSUS LOG FREQUENCY X VISCOSITY ....................................................... 14

FIGURE 10: Q VERSUS PERMEABILITY FOR DVORKIN AND NUR'S UNIFIED DYNAMIC POROELASTICITY MODEL (BIOT-SQUIRT FLOW) AND

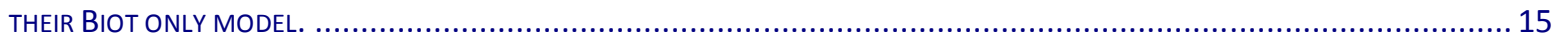

FIGURE 11: ATTENUATION VERSUS FREQUENCY WITH REGARD TO PORE ORIENTATION. ...................................................

FIGURE 12: TSVANKIN'S FIGURE 1 SHOWING HIS "SYMMETRY-AXIS" AND “ISOTROPY” PLANES IN A HTI SYSTEM............................. 17

FIGURE 13: BRAJANOVSKI'S FIGURE 2 SHOWING P-WAVE VELOCITY DISPERSION AND ATTENUATION FOR A FRACTURE SYSTEM OF VARYING

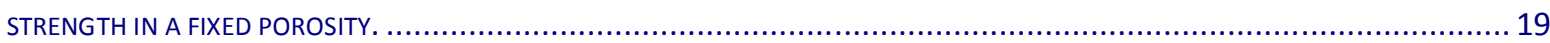

FIGURE 14: ChAKRABORTY'S FIGURE 5 SHOWING A COMPARISON OF THE STFT (B), THE CWT (C), AND THE MPD (D) METHODS. ....... 22

Figure 15: Location of the study area, the Charlton 30/31 Field in Northern Michigan Silurian Reef Trend. The STRUCTURE MAP SHOWN IS ON THE TOP OF THE SILURIAN NIAGARAN BROWN FORMATION, WHICH CONTAINS THE GUELPH FORMATION REEFS.

FIGURE 16: ISOTROPIC FREQUENCY ATTENUATION AS A RESULT OF A DRAINED, HIGH FLOW SYSTEM WITHIN THE MATRIX OF THE RESERVOIR.

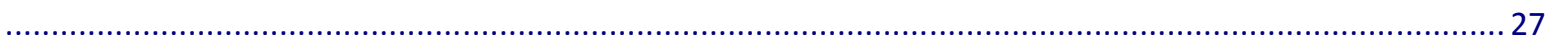

FIGURE 17: ANISOTROPIC FREQUENCY ATTENUATION AS A RESULT OF A LINEAR, DRAINED, HIGH FLOW SYSTEM RESULTING FROM OPEN, NATURAL FRACTURES.

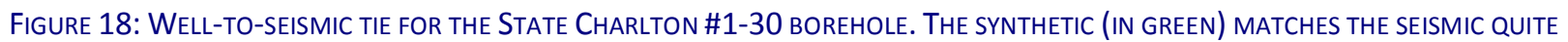

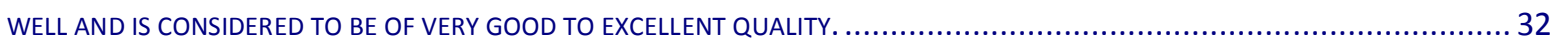

Figure 19: Structural Map of the Charlton 30/31 field on the top of the Guelph Formation................................... 35

FIGURE 20: ACQUISITION EXCLUSION ZONES FOR THE BASELINE 3D SEISMIC SURVEY. EXCLUSIONS OR LOW FOLD ZONES ARE HIGHLIGHTED

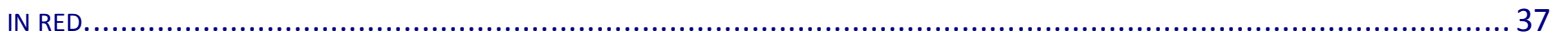

FIGURE 21: COMPARISON OF IN-LINE 1060 FROM THE 4D SEISMIC SURVEY. ....................................................42

Figure 22: ORIGINAL STRUCTURE MAP OF THE TOP OF THE GUELPH FORMATION BASED ON THE RESULTS OF DRILLING THAT TOOK PLACE

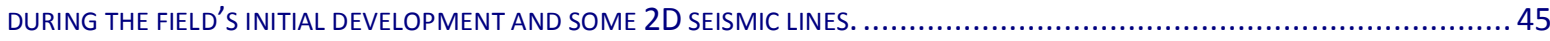

FIGURE 23: ORIGINAL STRUCTURE OF THE GUELPH REEF BASED ON EXISTING 2D SEISMIC AND WELL DATA AT THE START OF THE PROJECT. THIS WAS DIGITIZED INTO THE GEOFRAME PROJECT. ......................................................................... 46

Figure 24: Porosity log correlation SECTION THROUgh the CHARLTON 30/31 REEF. ..................................................46

FIGURE 25: AN ON-REEF / OfF-REEF COMPARISON OF ROCK PROPERTIES IN AND NEAR THE CHARLTON 30/31 FIELD..................... 47

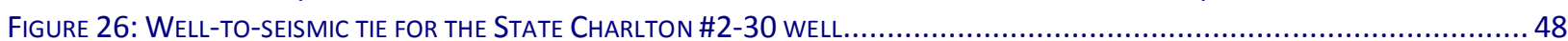

FIGURE 27: EXPLODED VIEW OF THE 12 LAYER FORWARD MODEL IN GEMINI CONSTRUCTED USING THE ROCK PROPERTIES SHOWN IN

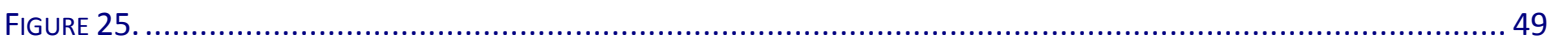

FIGURE 28: DISPLAY FROM ONE FORWARD 3D SEISMIC RAY-TRACE MODEL FOR MULTIPLE SOURCES FOR A SINGLE RECEIVER................ 50

FIGURE 29: FORWARD 3D SEISMIC RAY-TRACE MODEL FOR GATHER 91. ...........................................................

FIGURE 30: 3D RAY TRACE MODELING RESULTS SHOWING PREDICTED SEISMIC RESPONSE AT TOP OF REEF. WHILE MULTIPLE MODELING RUNS WERE PERFORMED ONLY THE RESPONSE OF THE LOWER UNITS ARE SHOWN IN THESE FIGURES. ................................ 51

FIgURE 31: TOPOGRAPHY AND FINAL ACQUISITION GEOMETRY OF THE BASELINE 3D SURVEY FOR THE CHARLTON 30/31 FIELD AREA. OPEN CIRCLES REPRESENT RECEIVER LOCATIONS AND THE PLUS SIGNS ARE ENERGY SOURCE POINT LOCATIONS. ..............................5 52

Figure 32: SCREEN CAPtURE OF PLATE 1 ILLUSTRATING THE RESUltS OF THE SHORT WINDOWEd WAVELET EXTRACTION. .................. 53 
FIGURE 33: THE SHORT WINDOWED WAVELET EXTRACTED FROM THE VICINITY OF THE STATE CHARLTON 2-30 WELL. 54

FiguRE 34: WELL TO SEISMIC TIE DEVELOPED WITH THE SHORT WINDOWED EXTRACTED WAVELET FOR THE STATE CHARLTON \#2-30 WELL

54

FIGURE 35: THE SHORT WINDOWED WAVELET EXTRACTED FROM THE VICINITY OF THE STATE CHARLTON 2-30 WELL AND THE SYNTHETIC

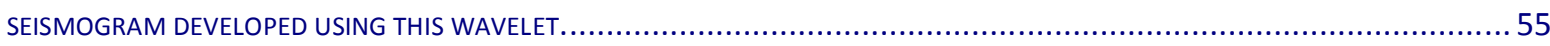

FiguRE 36: WELL TO SEISMIC TIE DEVELOPED WITH THE SHORT WINDOWED EXTRACTED WAVELET FOR THE STATE CHARLTON \#1-30 WELL.

FIGURE 37: THE SHORT WINDOWED WAVELET EXTRACTED FROM THE VICINITY OF THE STATE CHARLTON 2-31 WELL (OFF-REEF WELL) AND THE SYNTHETIC SEISMOGRAM DEVELOPED USING THIS WAVELET ...............................................................

FIGURE 38: WELL-TO-SEISMIC TIE ALONG A RANDOM LINE DEVELOPED WITH THE SHORT WINDOWED EXTRACTED WAVELET FOR THE STATE CHARLTON \#2-31 WELL AND THE SALLING HANSON \#1-31 WELL............................................................ 56

FIGURE 39: CORRELATION SECTION SHOWING ALL WELLS WITH SONIC LOGS. THESE WELLS WERE USE IT THE CONSTRUCTION ................ 57

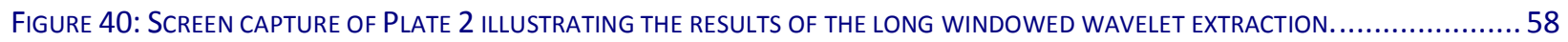

FIGURE 41: WAVELETS EXTRACTED FROM THE BASELINE 3D SURVEY USING A LONG WINDOWED EXTRACTION AND THEIR LOCATION WITHIN

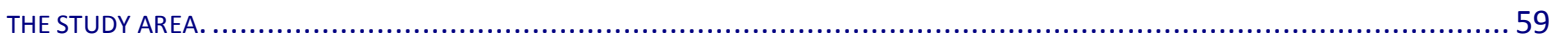

FIGURE 42: FREQUENCY POWER SPECTRUM FOR EACH WELL LOCATION EXTRACTED FROM THE VICINITY OF THE RESERVOIR. ................ 60

FIGURE 43: CROSSLINE THROUGH THE SEISMIC VOLUME AT THE REEF LEVEL SHOWING IMPORTANT SEISMIC HORIZONS INTERPRETED DURING

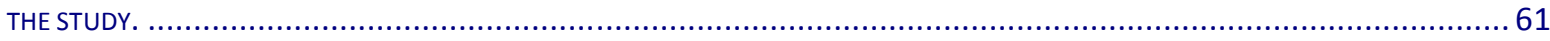

FiguRE 44: TIME AND DEPTH MAP FOR THE TOP OF THE GUELPH FORMATION. TIME CONTOURS ARE IN MILLISECONDS AND SHOWN IN

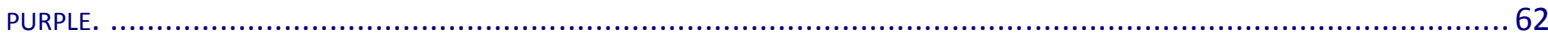

FIGURE 45: ISOPACH MAP Of THE SiLURIAN GUELPH REEF AT THE CHARLTON 30/31 FIELD. ................................................ 63

FIGURE 46: BLENDED SEISMIC ATTRIBUTE TIME SLAB 855 TO 860 MSEC. - HIGH VARIANCE (IN BLUE) AND HIGH AMPLITUDE (RED-

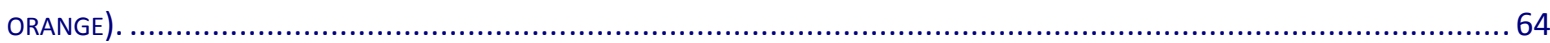

FIGURE 47: ILLUSTRATION OF METHOD USED FOR CONVERTING THE 2 MS TIME SLICES TO DEPTH USING THE TIME TO DEPTH RELATIONSHIP

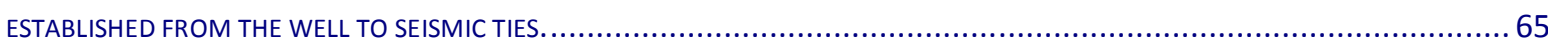

FIGURE 48: GAMMA RAY, POROSITY LOGS AND AVERAGE POROSITY VALUES BETWEEN DEPTHED 2 MS TIME SLICES FOR THE SALLING HANSON \#1-31 AND STATE CHARLTON "C"1-30 WELLS. ..................................................................... 66

FIGURE 49: ORIGINAL POROSITY LOGS (TRACK 1 (LEFT)) ARE PLOTTED FOR COMPARISON TO THE AVERAGE POROSITY COMPUTED OVER INTERVALS OF DEPTH CORRESPONDING TO 2 MSEC TIME INTERVALS (TRACK 2 9RIGHT) IN EACH WELL LOG STRIP.....................67

FIGURE 50: BLOCKED LOG POROSITY VALUES USED TO COMPUTE AN AVERAGE POROSITY WITHIN REEF FOR ALL WELLS IS PLOTTED VERSUS

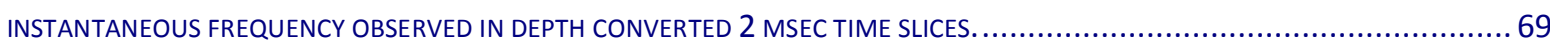

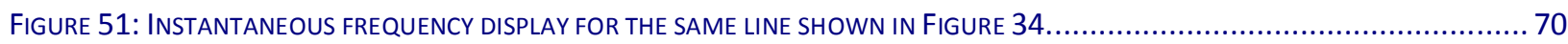

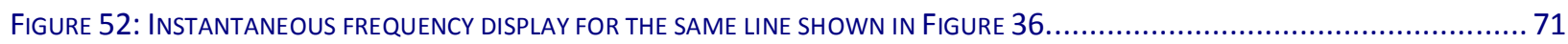

FIGURE 53: INSTANTANEOUS FREQUENCY DISPLAY FOR THE SAME LINE SHOWN IN FIGURE 38.......................................... 71

FiguRE 54: Porosity DISTRIBUTION MAP FOR TIME SLICE 889 (LEFT) AND THE MAP OF INSTANTANEOUS FREQUENCIES (RIGHT) ON WHICH

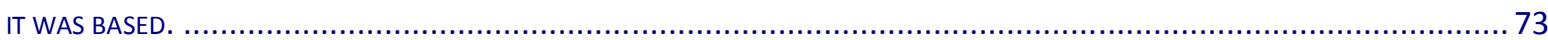

FiguRE 55: INSTANTANEOUS FREQUENCY DISPLAY FOR IN-LINE 80 THROUGH THE STATE CHARLTON 1-30 WELL USING THE UPDATED INSTANTANEOUS FREQUENCY EXTRACTION PARAMETERS (ON LEFT) AND THE INITIAL SECTION (ON RIGHT). NOTE THAT THESE SCREEN

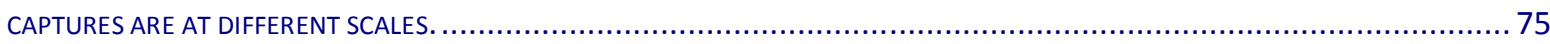

FIGURE 56: CROSS SECTION OF SIMULATION SHOWING THE DISTRIBUTION OF RESERVOIR GRID CELLS IN THE MODEL AND SEISMIC BASED

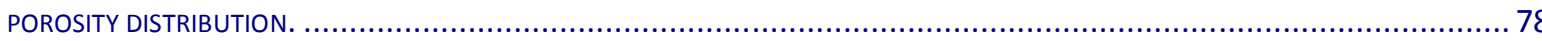

FIGURE 57: HISTORY MATCH SHOWING 18 YEARS OF FIELD GOR (GAS TO OIL RATIO) HISTORY, DASHED LINE, AND SIMULATED GOR, SOLID LINE.

FIGURE 58: POROSITY CONFIGURATION SHOWN AT LEFT HAS ITS OVERALL BULK POROSITY DECREASED IN THE DISPLAY ON RIGHT BUT THE DISTRIBUTION OF THE POROSITY ZONES REMAINS THE SAME.

Figure 59: Predicted oll distribution Within the Charlton 30/31 ReEf for K Slice 9 at time Step September 1, 2007 from the PREDICTIVE, FORWARD-LOOKING RESERVOIR SIMULATION.

Figure 60: Predicted CO2 saturation distribution Within the Charlton 30/31 ReEF for K slice 9 at times Step September 1, 2007 FROM THE PREDICTIVE, FORWARD-LOOKING RESERVOIR SIMULATION. ................................................... 83

Figure 61: Percent amplitude difference for A2-CARbonate BetWeen Monitor ANd Baseline SurVeys.......................... 85 FIGURE 62: 4D AMPLITUdE DIFFERENCE FOR THE A2-CARBONATE. DifFERENCES ARE PLOTTED AS A PERCENT AMPLITUDE DIFFERENCE MAP (Monitor-BASELINE) WITH RESPECT TO 100\% DIFFERENCE. DARK GRAY AREAS EXCEED 100\% DifFERENCE. Colored AREAS HAVE DifFERENCE OF LESS THAN 100\%.DIfFERENCE FOR A2-CARBONATE BETWEEN MONITOR AND BASE SURVEYS. 
Figure 63: Crossline 5045 (Baseline survey above, Monitor survey below) flattened on the top of the A2 Carbonate. LOCATION OF THE CROSSLINE IS SHOWN AS A BLUE LINE ON THE PERCENT AMPLITUDE DIFFERENCE MAP FOR THE FLATTENED A2 CARBONATE EVENT AT RIGHT. DARK GRAY COLOR INDICATES PERCENTAGES BEYOND 100\%

Figure 64: CRossline 5045 (BASEline SURVEy ABOVE, Monitor SURVEY BELOW) FLATTENEd on the tOP OF THE A2-CARBONATE WITH SINGLE SAMPLE AMPLITUDE ANNOTATED.

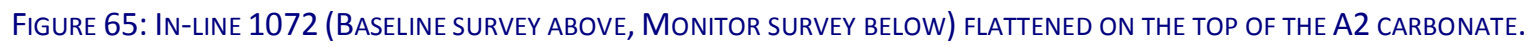
LOCATION OF THE CROSSLINE IS SHOWN AS A BLUE LINE ON THE PERCENT AMPLITUDE DIFFERENCE MAP AT RIGHT. DARK GRAY COLOR INDICATES PERCENTAGES BEYOND 100\%.

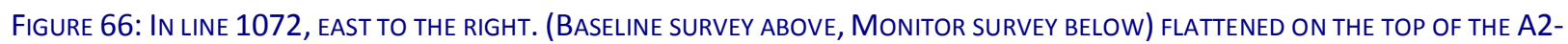

CARBONATE WITH SINGLE SAMPLE AMPLITUDE ANNOTATED.

Figure 67: TIME SLICE 15 MS FROM the A2-CARBONATE FLATTENEd MONITOR SURVEY SHOWING A HIGH AMPLITUDE ANOMALY" JUST EAST OF THE INJECTION POINT "JETTING" TOWARD THE ENHANCED OIL RECOVERY PRODUCTION WELL................................96

FIgURE 68: COMPARISON BETWEEN TIME SLICE 15 MS FROM THE A2 CARBONATE ON BOTH BASELINE AND MONITOR SURVEYS. .......... 97

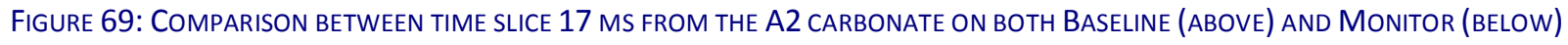
SURVEYS.

Figure 70: TIME SLICE 20 MS From the A2-CARBonAte Flattened MONITOR SURVEY SHOWING that THE HIGH AMPLITUDE ANOMALY APPEARS TO BE WEAKENING IN STRENGTH BUT STILL SUGGESTING A HORSESHOE SHAPED ORIENTATION AROUND THE AREA IMMEDIATELY WEST OF THE STATE CHARLTON \#1 - 30 WELL. 100

Figure 71: TOP - tIME SLICE 15 MS BELOW THE FLATTENEd A2-CARBONATE. BOtTOM - LAYER 9 fROM THE FINAL, PREDICTIVE RESERVOIR SIMULATION (TIME STEP SEPTEMBER 2007) WHICH CORRESPONDS TO THE APPROXIMATE DEPTH OF THE TIME SLICE. 102

Figure 72: TOP - tiME SLICE 16 MS BeloW tHE FLATTENEd A2-CARBONATE. BotTOM - LAYER 10 FROM THE FINAL, PREDICTIVE RESERVOIR SIMULATION (TIME STEP SEPTEMBER 2007) WHICH CORRESPONDS TO THE APPROXIMATE DEPTH OF THE TIME SLICE. 103

FIGURE 73: SAME DISPLAY SHOWN IN FIGURE 71 EXCEPT IMAGE ADDED INTO THE UPPER RIGHT TO SHOW THE TIME SLICE OVERLAIN WITH GRID CELLS FROM THE PREDICTED RESERVOIR SIMULATION. SEE TEXT FOR FURTHER EXPLANATION..... 105

FIGURE 74: ILLUSTRATION OF MATRIX AND FRACTURE POROSITY / PERMEABILITY ZONES WITHIN THE REEF. 109

FIGURE 75: AZIMUTHAL VOLUMES ARE DEVELOPED BY BINNING ALL OF THE SOURCE / RECEIVERS WITHIN A SPECIFIC ANGULAR SWATH.. 110

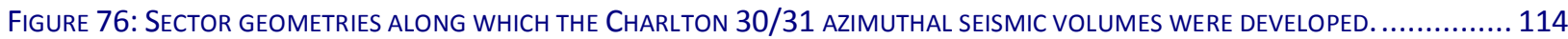
FIGURE 77: NORMALIZATION OF THE ISO-FREQUENCY VOLUMES IN SPECIFIC AZIMUTHS TO PRODUCE THE SELECTED FREQUENCY FRACTION (SFF) VOLUMES. 116

FIGURE 78: NORMALIZATION OF THE ISO-FREQUENCY VOLUMES OF A SPECIFIC FREQUENCY TO PRODUCE THE ALL AZIMUTH FRACTION (AAF) VOLUMES.

FIGURE 79: NORMAL AND FLATTENED IN-LINE 1073 FOR THE $160^{\circ}$ AZIMUTHAL SEISMIC VOLUMES. 121

FIGURE 80: IN-LINE 1072 THROUGH FLATTENED A2 CARBONATE VOLUME FROM THE MONITOR SURVEY. THE AMPLITUDE ANOMALY WITHIN THE RESERVOIR INTERVAL IS LOCATED ALONG THE TOP OF THE REEF, CIRCLED IN WHITE. THE ANOMALY IS INTERPRETED TO RESULT FROM CHANGES OF IMPEDANCE RELATED TO CO2 FLOODING OF THIS INTERVAL. THE TOP AND BASE OF THE REEF ARE SHOWN AS BLUE LINES. 122

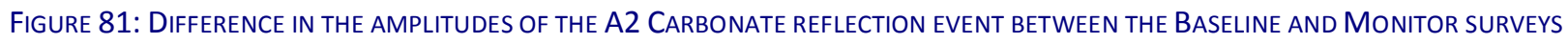
(BASELINE -MONITOR). LOCATION OF AZIMUTHAL SEISMIC STUDY IS CIRCLED IN RED. 123

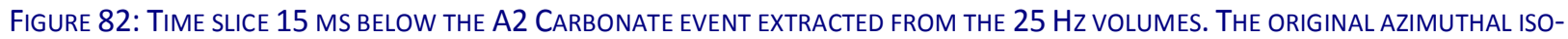
FREQUENCY VOLUME IS SHOWN ON THE LEFT AND THE ALL AZIMUTH FRACTION (AAF) VOLUME IS SHOWN ON THE RIGHT. ........ 124

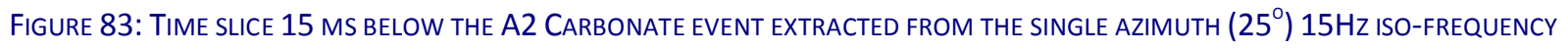
(IF15A25) ON LEFT AND THE 25Hz ISO-FREQUENCY (IF25A25) ON RIGHT. .............................................. 125

Figure 84: A comparison of the Selected Frequency Fraction iso-frequency Volumes 65 Hz (SFF65A70) to 15 Hz (SFF15A70) IN $10 \mathrm{HZ}$. INTERVALS FOR THE $70^{\circ}$ AZIMUTH,

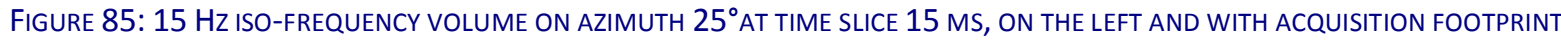
LINEATIONS INTERPRETED ON THE RIGHT IN BLUE.

Figure 86: All Azimuth Fraction Sum Volumes deVeloped during the construction of the All Azimuth Fraction (AAF) VOLUMES FOR 55 HZ ON LEFT (AAF55SUM) AND 15 HZ ON RIGHT (AAF15SUM).

FIGURE 87: $15 \mathrm{HZ}$ AND 30Hz ISO-FREQUENCY SECTIONS USED BY ODEBEATU (2006) TO DETECT FREQUENCY ATTENUATION ASSOCIATED WITH A RESERVOIR IN A GAS FIELD IN THE NORTH SEA.

Figure 88: 15 to 55 Hz All Azimuth Fraction Sum Difference Volume 15 mS Below the A2 Carbonate.... 
FIGURE 89: ISO-FREQUENCY SUM DIFFERENCE VOLUME FOR 15 HZ MINUS 55 HZ AT TIME SLICE 15 MS IN THE NORTHERN PORTION OF THE CHARLTON 30/31 OIL FIELD SUBTRACTED FROM the ISO-FREQUENCY SUM DifFERENCE VolUME FOR 55 Hz (SEE FIGURE 88)

OVERLAYING WITH THE CELLS PREDICTED TO BE $\mathrm{CO}_{2}$ FILLED BY THE FORWARD-LOOKING RESERVOIR SIMULATION.

FIGURE 90: 15 HZ ISO-FREQUENCY VOLUME FROM AZIMUTH 25 (IF15A25) AT TIME SLICE 15 MS..................................... 135

FIGURE 91: ISO-FREQUENCY 15 HZ RATIO VOLUME, AZIMUTH 70 , (IF15A70) TIME SLICE 15 MS. ..................................... 136

FIGURE 92: ISO-FREQUENCY 15 HZ RATIO VOLUME, AZIMUTH 115 , (IF15A115) TIME SLICE 15 MS. ................................... 137

FIGURE 93: ISO-FREQUENCY 15 HZ RATIO VOLUME, AZIMUTH 160 , (IF15A160) TIME SLICE 15 MS..................................... 138

Figure 94: AZIMUTH 25 $5^{\circ}$, ISO-FREQUENCY 15 Hz, TIME SLICE 15 MS (LEFT) AND AZIMUTH 115 , ISO-FREQUENCY 15 Hz, TIME SLICE 15 MS (RIGHT). SEE TEXT FOR ANNOTATION EXPLANATION.................................................................... 139

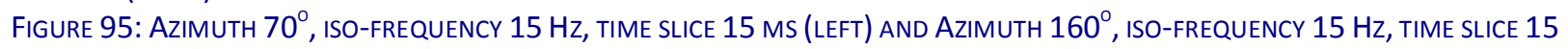

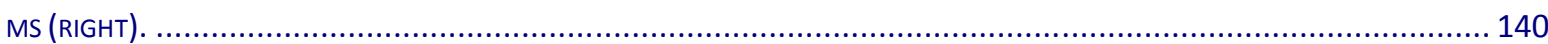

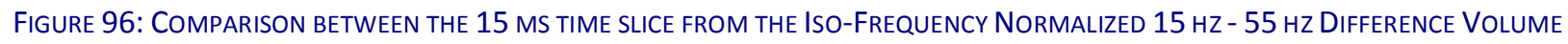
of the Baseline Azimuthal SurVeys And the 15 mS time SLICE from the flattened 4D Monitor SuRVey Amplitude

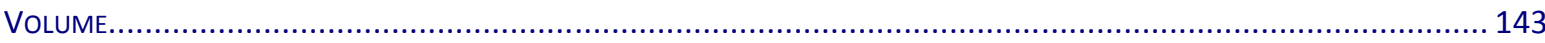

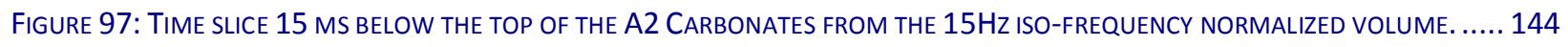

FIGURE 98: COMPARISON BETWEEN ISO-FREQUENCY NORMALIZED 15 HZ VOLUME FOR AZIMUTH 70 (A) AND MONITOR SURVEY

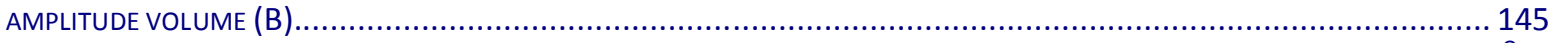

FIGURE 99: 15MS TIME SLICE FROM THE NORMALIZED $15 \mathrm{HZ}$ ISO-FREQUENCY VOLUME. THE TIME SLICE WAS EXTRACTED FROM THE $25^{\circ}$ AZIMUTH (A) AND $160^{\circ}$ (B). FIGURES A2 AND B2 SHOW INTERPRETED FEATURES (GREEN) EXTENDING NE FROM THE END OF THE State Charlton "C" 2-30 lateral.

FIGURE 100: COMPARISON OF IF15A70, ISO-FREQUENCY 15 HZ, AZIMUTH 70 FOR TIME SLICE 15 MS (LEFT) AND IF15A160, ISOFREQUENCY $15 \mathrm{HZ}$, AZIMUTH $160^{\circ}$ FOR TIME SLICE $15 \mathrm{MS}$ (RIGHT).

Figure 101: Fracture Characterization Model \#1 (on Right) DEVELOPED by COMPARING FEATURES OBSERVED ON IF15A160, (15 HZ ISO-FREQUENCY, AZIMUTH 160 VOLUME) AT TIME SLICE 15 MS (SHOWN AT LEFT) WITH AZIMUTHALLY NORMALIZED 15 HZ ISOFREQUENCY, AZIMUTH 70 VOLUME. SEE TEXT FOR EXPLANATION.

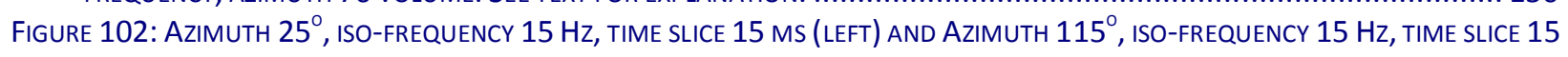

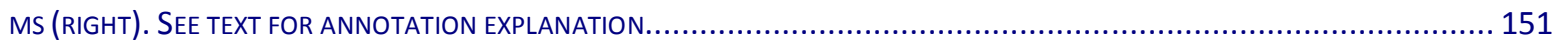

FIgURE 103: Fracture ChARACTERIZATION MOdEL \#2 DEVELOPED BY COMPARING FEATURES OBSERVED ON AZIMUTHALLY NORMALIZED 15 HZ ISO-FREQUENCY, AZIMUTH 25 VOLUME AT TIME SLICE 15 MS WITH AZIMUTHALLY NORMALIZED 15 HZ ISO-FREQUENCY,

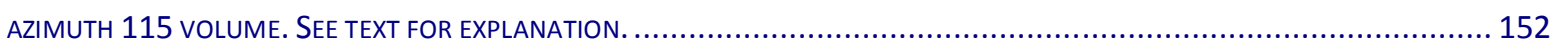

Figure 104: Combined Fracture Model Resulting from the Merging of Fracture Characterizations \#1 and \#2........... 153 Figure 105: Comparison of the Baseline (A) ANd Monitor (B) SURVEYS at time SLICE 15 MS BeloW flatten A2CARBonate AND THE LOCATION OF AREAS WITH HIGH REFLECTION STRENGTH FROM ALL FOUR AZIMUTHAL ISO-FREQUENCY VOLUMES (C).......... 155 


\section{CHAPTER 1: OVERVIEW}

Prior to the drilling a borehole into the subsurface and measuring various reservoir parameters directly using well logs and/or cores, the only methods available to obtain information on potential reservoirs are geophysical in nature. Of these methods 3D surface seismic surveys are often preferred as their resolution and overall accuracy are typically better than other methods, such as gravity and magnetic surveys. For many years 3D surface seismic data have provided accurate information concerning the structural and stratigraphic framework of the subsurface. However, modern seismic interpretation software packages, which were first developed in the 1980s and are currently widely available on powerful workstations, now allow for the extraction of significant amounts of stratigraphic information from the reservoir utilizing these data sets.

Subsurface reservoirs often display changes in many of their characteristics. Among the more important of these reservoir characteristics are porosity and permeability. For porosity the geometry of the pore space can be an important factor on how the reservoir responds to various stimuli. Within carbonate reservoirs zones of high matrix porosity, such as vugs, can elicit responses different than zones of open natural fractures. The ability to identify zones within a subsurface reservoir that have higher porosity/permeability and to characterize them according to their geometries before the drilling of an expensive borehole would be of great significance when planning various types of projects. Projects, such as those regularly performed in oil and gas field development, enhanced oil recovery projects and $\mathrm{CO}_{2}$ sequestration projects, would benefit greatly from this type of capability.

The detection and characterization of these high porosity/permeability zones may be possible utilizing selected seismic attributes and seismic data sets limited by their source-receiver orientation. Previous work has indicated that high porosity/permeability zones within a reservoir can have a direct effect on seismic frequencies and velocities. It may be possible to utilize these attributes to identify these zones. Additionally, certain porosity/permeability geometries, such as open natural 
fractures, have been shown to cause anisotropy for some reservoir parameters and produce observable effects in some seismic attributes. Analyzing the response of these seismic attributes within azimuthally-limited seismic data would reveal the nature of the anisotropy within a porosity/permeability system. Processing of normal 3D seismic surveys occur by summing (or "binning") multiple traces obtained from all of the available shot-receiver pairs in the survey. A resulting stacked trace on any single "Common Mid Point (or CMP) then contains information assembled from all directions (or azimuths) in the "full fold" portion of the survey. Such 3D surveys are usually referred to as normal, "Rich-Azimuth" or "Full-Azimuth" surveys.

In addition to obtaining structural information about the reservoir some stratigraphic features are often observable with 3D seismic data. Lithologies and depositional environments can often be interpreted within conventional 3D seismic volumes, however, locating and mapping important reservoir parameters, such as high porosity/permeability zones and open natural fractures is an area of current research interest. Previous investigators have demonstrated mathematically and experimentally that zones of high porosity are generally slower (depending on fluid content) and result in lowered frequency response through the attenuation of higher frequencies in the transmitted wavefield. It should be noted that the vast majority of this mathematical and experimental work has been focused on frequencies well outside those normally encountered while working with traditional surface seismic (generally between 6 to $120 \mathrm{~Hz}$ ). These theoretical studies typically investigate frequencies in the kilohertz range or higher. In addition to these theoretical studies some field studies have been published that demonstrate these effects with regard to velocity and shear-wave splitting. However, while numerous theoretical and experimental publications exist only a very few studies have been published that demonstrate the use of frequency analysis for porosity/permeability characterization using actual field data. Personal communication from various individuals in the geophysical sciences have indicated to me that within the oil and gas industry Wavelet Induced Fluid Flow (WIFF) has been accepted as a main cause of low frequency zones associated with high 
porosity/permeability zones. These low frequency zones are produced due to the attenuation of high frequencies. Muller, in his 2010 paper in Geophysics entitled "Seismic wave attenuation and dispersion resulting from wave-induced flow in porous rocks - a review", summarized the main theoretical and experimental works of the past few decades in the area. In his abstract Muller states "It is believed that for frequencies below $1 \mathrm{kHz}$, the most important cause (of elastic wave attenuation in heterogeneous porous media) is the wave-induced flow between mesoscopic inhomogeneties, which are large compared with the typical individual pore size but small compared to the wavelength." Attenuation occurs due to the friction generated heat produced by the fluid flow. This is sometimes referred to as "Intrinsic Attenuation" and is frequency dependant due to the variation in the timing between the compression and extension phases of a wavelet passage through the reservoir. Muller's figure \#2, shown here as this paper's Figure 1, illustrates this effect. Here T/2 and T are directly related to wavelength and, thereby, frequency. For high frequencies the amount of time between 0 and $\mathrm{T}$ will be fairly
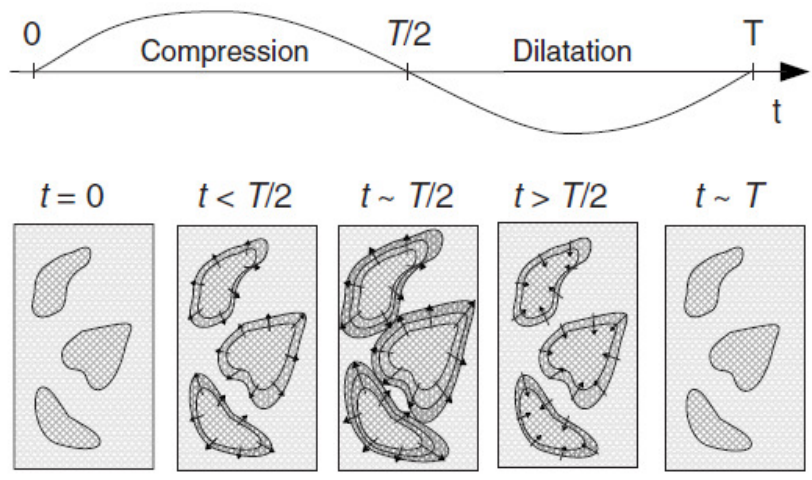

Figure 1: Muller's figure \#2 illustrating the compressional and extensional phases of a wavelet passage through a porous media.

short, whereas, for lower frequencies 0 to $\mathrm{T}$ will be longer. Therefore, for higher frequencies more compressional / extensional cycles will occur during the same time period than for lower frequencies. The amount of energy lost due to heat generation is greater for the higher frequency component of the seismic signal than the low frequency component. Therefore, in zones of high porosity / permeability the lower frequency component of the seismic signal is preserved whereas the higher frequency component is removed due to attenuation. Frequency attenuation is believed to be particularly significant in partially fluid saturated rocks where small amounts of gas provide the high compressibility needed to accommodate fluid flow during these compressional / extensional cycles. 
Muller states that "The frequency dependence of wave velocity and attenuation in a partially saturated medium is controlled by the size, shape, and spatial distribution of the fluid pockets; the permeability and elastic moduli of the solid matrix; and the properties of the two fluids."

In addition to Wave Induced Fluid Flow, fracture compliance has also been shown to be a cause of frequency attenuation. Chapman (2003) reported that "frequency-dependent anisotropy can occur in the seismic frequency band when larger fractures are present." and that "Strong anisotropic attenuation can occur in the seismic frequency band." Characteristics that contribute to the response of a fractured reservoir to a passing seismic wave include the number of fractures and their special distribution (density), their aperture, the porosity / permeability of the matrix that line the walls of the fractures, the viscosity of the fluid or gas in the fractures and matrix porosity as well as the angle of incidence of the seismic signal with respect to the fracture. Frequency changes in fractured reservoirs have been theorized to range from low seismic frequencies to high ultrasonic frequencies. Seismic attenuation within fractured reservoirs is the result of "Intrinsic Attenuation" and Scattering or “Apparent Attenuation.”

Ali (2011) modeled the effects of a single, mesoscopic fracture set on p-wave velocity and attenuation azimuthally for a single angle of incidence with respect to the fracture set. Figure 2 shows his results (modified from Ali's figure \#7) for an angle of incidence of $40^{\circ}$ with respect to a single fracture set. The azimuthal orientation of the mesoscopic fracture set is also $40^{\circ}$. The response in different seismic frequencies is shown at various azimuths in colors corresponding to black for 20 $\mathrm{Hz}$, blue for $45 \mathrm{~Hz}$, red for $65 \mathrm{~Hz}$ and green for $90 \mathrm{~Hz}$. As can be seen the maximum attenuation occurs at $130^{\circ}$ azimuth, which is perpendicular to the fracture trend. This is also the same azimuth where the minimum velocity occurs. The velocity minimums and attenuation maximums occur at the same azimuth for all frequencies; however, the amount of attenuation is greater for the higher frequencies. For the $65 \mathrm{~Hz}$ frequency the difference of attenuation between orientations that are parallel and perpendicular to the fracture set trend is .0425 whereas, for the $20 \mathrm{~Hz}$ frequency this 
difference is only .025. Therefore, Ali demonstrates that greater attenuation occurs in the higher frequencies and that the greatest amount of attenuation occurs perpendicular to the fracture trend.
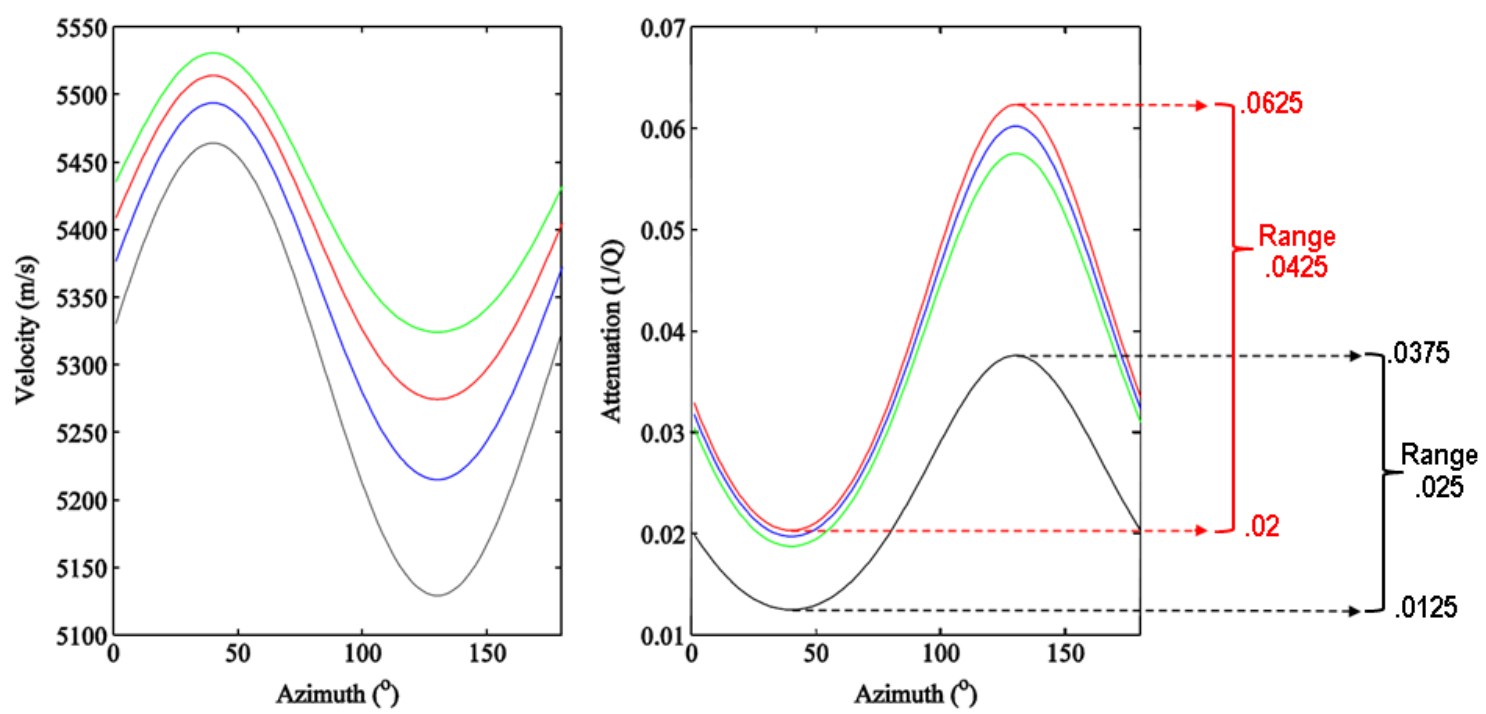

Figure 2: Azimuthal changes in velocity and attenuation with respect to a single mesoscopic fracture set for frequencies $20 \mathrm{~Hz}$ (black), $45 \mathrm{~Hz}$ (blue), $60 \mathrm{~Hz}$ (red) and $90 \mathrm{~Hz}$ (green). Modified from Ali (2011) for an angle of incidence of $40^{\circ}$ with respect to the fracture set.

Given the anisotropic nature of attenuation related to fracture compliance due to scattering (or "Apparent Attenuation") and the isotropic nature of attenuation related to WIFF (or "Intrinsic Attenuation") in high matrix porosity/permeability it should be possible to identify zones within a reservoir using azimuthally-binned seismic volumes where the geometry of the attenuation is used to distinguish between these two porosity / permeability systems. Using spectral decomposition isofrequency volumes can be developed from azimuthal seismic volumes, normalized and then evaluated to determine where strong low frequencies exist. If these strong low frequency zones correspond to other indicators of high porosity / permeability then a high probability would exist that they result from attenuation.

As part of a recently completed project which was fully funded by the US Department of Energy (DOE Grant No.: DE-FC26-04NT15425, Brian Toelle, Principal Investigator), I acquired 4D surface seismic for the purpose of monitoring a $\mathrm{CO}_{2}$ enhanced oil recovery effort in a northern 
Michigan oil field. This field, the Charlton 30/31 field, which produces from a pinnacle reef located in the Northern Silurian Reef Trend of the Michigan Basin, ceased its primary production of oil in 1997. The acquisition of the initial "Baseline" 3D of the 4D survey occurred in March of 2004 in preparation for the enhanced oil production operations. In addition to the normal seismic processing sequence performed following acquisition, specialized processing was performed on this Baseline 3D survey in order to develop multi-azimuthal 3D volumes.

Multi-azimuthal 3D volumes (also known as "azimuthal" 3D volumes) are constructed by binning shot-receiver pairs sharing a narrow range of azimuths. These common azimuth volumes provide information about variability in acoustic properties as a function of the raypath's azimuth. For instance, if zones of high matrix porosity/permeability are elongated in one direction, such as with an aligned fracture trend, then one would expect the seismic signal's low frequency content reflected from the reservoir to be greater for source receiver azimuths oriented perpendicular to the length of the fracture rather than parallel it. Zones of lower frequency response are mapped using spectral decomposition of the 3D volumes. Spectral decomposition of the Charlton 30/31 oil field baseline (pre-injection) 3D survey was performed on the azimuthally processed 3D volumes in order to develop several iso-frequency volumes. These were used for interpretation.

The intensity of certain seismic responses have also been shown to vary dependent upon whether the porosity/permeability system is "drained" or "undrained". Undrained porosity/permeability systems, or pore systems where pore fluid movement is prevented when a compressive seismic wave passes, are considered "stiffer" (having a higher bulk modulus) than drained porosity/permeability systems. Drained porosity/permeability systems allow greater amounts of fluid movement due to the presence some amounts of gas within the system or a connection to pore systems that are uncompressed at that time.

High matrix porosity/permeability zones, identified in this Silurian Reef using well logs, are azimuthally isotropic in nature and observable on all azimuthal ray paths through the reservoir. Open, 
natural fracture systems represent zones of high porosity/permeability. Unlike matrix porosity individual fracture sets are generally linear and trend along a mean azimuth with small standard deviation. In this case, one expects P-wave attenuation in the direction perpendicular to an open fracture trend. These linear features should produce anisotropy in the azimuthal spectral response.

During the primary production from the Charlton 30/31 oil field a number of the wells began to produce gas as the reservoir pressure decreased. Therefore, during the time this research was conducted the reef contained gas which would have provided accommodation space for fluid movement induced by the compressional seismic energy. Therefore, the reef's porosity/permeability system is considered to be a drained porosity/permeability system that permits fluid flow with the passing of compressional seismic waves. For the remainder of this document this system within the reef will be referred to as the "drained flow system".

During the examination of the Charlton 30/31 Baseline 3D survey, iso-frequency volumes, developed from the azimuth-limited 3D seismic volumes were used to identify and locate zones of strong lower frequencies. Those found on all of the azimuth-limited volumes are interpreted to be associated isotropic zones of high matrix porosity and, therefore, permeability. Those identified only along certain azimuths reveal anisotropy in the wavefield interpreted to result from open natural fracture trends. These observations were used to map their location and trend. This approach to characterizing drained flow systems within reservoirs is completely new and unique.

Overall, this research resulted in three separate but related studies that are represented in this dissertation in chapters 4, 6 and 7. In Chapter 4, "Frequency Attenuation and Its Relationship to Drained, High Flow Systems" I present my theory that frequency attenuation occurs in zones with high matrix porosity (drained, high flow systems), which are indicated by the presence of stronger low frequencies. I support this with data from the full-azimuth baseline survey and well log data. 
In Chapter 6, "Baseline Survey Azimuthal Iso-Frequency Interpretation”, I extend this concept to include aligned, drained, high flow systems (open fracture trends) through the interpretation of azimuth-limited iso-frequency seismic volumes produced through spectral decomposition. This interpretation methodology has not been previously described in the literature to date and offers a unique approach for discriminating between high matrix and fracture related drained high flow systems. This also allows the reef's drained, high flow system to be characterized to a far greater degree than previously possible.

In Chapter 7, "Drained, High Flow System Characterization and Comparison with 4D Seismic", I compare the results of the analysis described in Chapter 6 with the results from the 4D seismic analysis performed for the Department of Energy study. The results of this comparison are based on amplitude difference mapping and reservoir simulation predictions. These findings support the characterization developed in Chapter 6.

The main consequence from the positive outcome of this research is the development of a seismic interpretation methodology that can be used to identify potential zones of high rate oil or gas production in reservoirs using conventional p-wave seismic data alone. The identification of open fracture trends and zones of high matrix porosity/permeability before drilling is of significant advancement in borehole placement and will lead to greater production rates and better economics in the oil and gas industry due to the increased production from the reservoir. Additionally, environmentally related efforts, such as $\mathrm{CO}_{2}$ sequestration projects, will also benefit through the identification of zones that would permit higher injection rates. It should be noted that this study is not a theoretical, experimental or mathematical based study but rather one of the first studies to attempt to demonstrate these concepts using actual field (surface seismic) data. 


\section{CHAPTER 2: INTRODUCTION}

\subsection{Statement of the Problem}

Numerous human activities currently exploit the Earth's subsurface as part of their normal routine. These include oil and gas exploration and field development, enhanced oil recovery, water resource management, underground gas storage as well as some forms of waste and wastewater disposal. With growing concerns over greenhouse gas emissions and global warming the sequestration of large amounts of $\mathrm{CO}_{2}$ produced by power generation facilities and ethanol production plants will soon be added to this list. All of these activities require locating and exploiting drained flow systems within the reservoir rocks, which have high enough capacity to support these activities. The ability to accurately detect and characterize high flow systems within the reservoir prior to the drilling of expensive boreholes would represent a significant advancement of the current technology and could save these industries many millions of dollars in the cost of dry holes or poor performing wells.

The seismic interpretation methodologies presented in this study are specifically designed to address this characterization issue. In order to demonstrate these methodologies, I have identified, interpreted and mapped zones of low frequency on azimuthal seismic volumes. This was performed utilizing instantaneous frequency and iso-frequency volumes developed through spectral decomposition. As mentioned in the previous chapter, zones of low frequency have been shown to correlate with zones of increased attenuation due to high porosity/permeability that exist in the rock matrix. Additionally, open fractures cause the attenuation of higher frequencies due to fracture compliance. Comparison of these zones on the different azimuthal volumes determined where the attenuation was isotropic or anisotropic in nature. Zones of isotropic frequency attenuation are shown to be attributed to increased porosity/permeability and rock matrix. Zones of anisotropic frequency attenuation are believed attributed to the presence of open natural fractures. These are identified by differences associated with the predicted reservoir simulation (which is based on the interpretation of 
matrix attributes) and the results of the 4D amplitude difference mapping. The orientation of these fracture trends have been determined based on the geometry of the observed attenuation.

The results of this mapping of the drained flow systems were then compared with the results of the $4 \mathrm{D}$ difference mapping. Zones of major amplitude differences within the reservoir that were observed between the "Baseline 3-D" and the "Monitor 3-D" were compared to this azimuthal, isofrequency based drained flow system characterization. The strong correspondence of amplitude difference with the zones interpreted by the above described method is interpreted as confirmation of this methodology. The weak correspondence between time-lapse amplitude differences with the interpreted zones of increased porosity suggests that the relationship does not accurately predict areas of increased oil recovery and $\mathrm{CO}_{2}$ flooding. The geometry of the observed amplitude anomalies will provide information about the geometry of reservoir features associated with zones of increased matrix porosity/permeability or open natural fractures.

\subsection{Background Information}

2.2.1 Previous Work - High Frequency Attenuation as an indicator of Porosity / Permeability

A significant amount of work has been done on the subject of attenuation as a porosity / permeability indicator. The vast majority of this has been theoretical, based on mathematical model simulations. Additionally, most of this work has focused on attenuation as inferred from changes in velocity or reflection amplitude. Only a very few publications have dealt with the actual application of frequency attenuation using real surface seismic data for the purpose of identifying porosity / permeability.

The initial work was published by M. A. Biot in his papers, "Theory of Elasticity and Consolidation for a Porous Anisotropic Solid" (1955) and "Theory of Deformation of a Porous Viscoelastic Anisotropic Solid" (1956), both in the Journal of Applied Physics. These papers have repeatedly been cited by many workers since. 
An early effort to apply this work to the geosciences was performed by H. C. Misra in 1965 with the publication of a thesis entitled "Permeability of porous media to transient flow". In this work Misra theorized that "the permeability of the porous medium, as it occurs in the equations of motion, is frequencydependent." During this investigation Misra found that permeability "approach(es) the static or Darcy value at low frequencies". Gregory (1977) in his paper titled "Aspects of Rock Physics From Laboratory and Log Data that are Important to Seismic Interpretation" investigated the relationship between rock physical

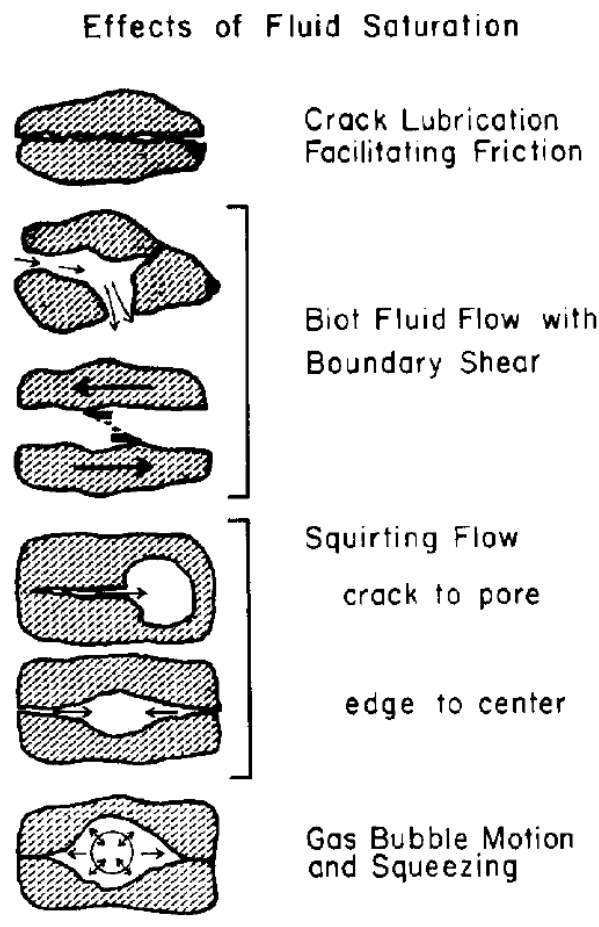

Figure 3: Attenuation mechanisms discussed by Johnston et al, 1979. properties and the influence of the subsurface environment as they related to typical seismic stratigraphy problems. Gregory concluded that "The magnitude of viscous losses predicted at higher frequencies for rocks with relatively high permeability may be large enough to measure with suitable experimental techniques". Johnston, Toksoz, and Timur (1979) discuss various attenuation mechanisms, shown in Figure 3, in their paper "Attenuation of seismic waves in dry and saturated rocks: II. Mechanisms". They obtained data through various experimental methods,

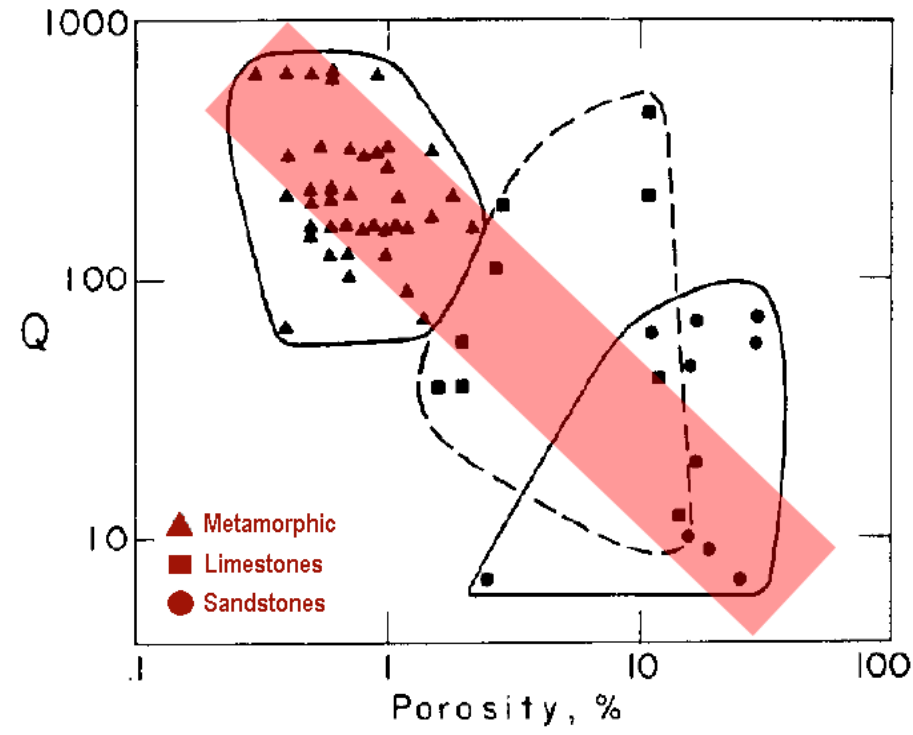

Figure 4: Attenuation trends as a function of porosity taken from Bradley and Fort (1966). 
discussed in volume 1 (Toksoz et al, 1979), including pulse transmission of several types, resonant bars, and slow stress cycles, as well as data published by other workers. In their Figure \#1 which was based on work performed by Bradley and Fort, 1966, (shown here in this paper's Figure 4 slightly modified to illustrate the data trend), "a general trend of Q inversely proportional to porosity" can be observed in various lithologies.

Based on their review they concluded that in saturated rocks under normal conditions found within the earth, friction on thin cracks and grain boundaries is the dominant attenuation mechanism at ultrasonic frequencies. At lower frequencies "Squirting" was suggested as a mechanism in saturated porous mediums. Figure 5 shows a comparison of various attenuation mechanisms that they investigated.

Palmer and Traviolia in their 1980 paper "Attenuation by Squirt Flow in

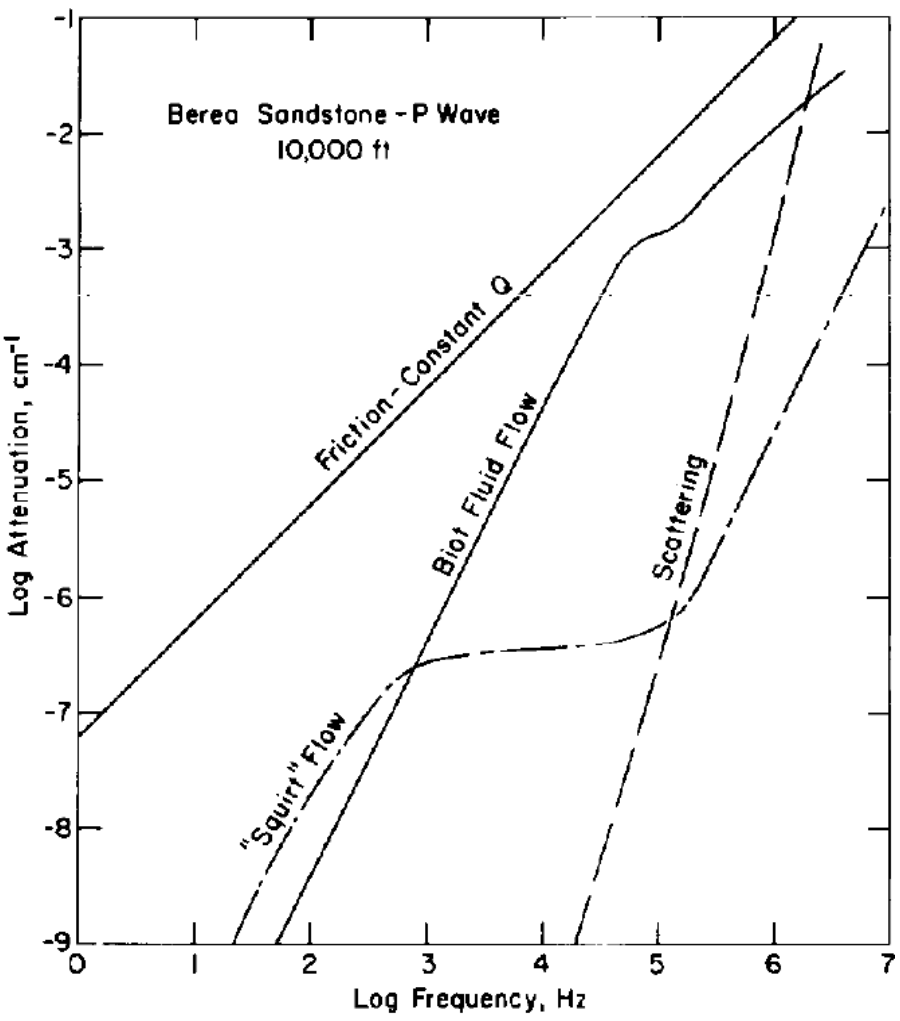

Figure 5: Johnston et al, 1979 figure \#14 showing P-wave attenuation coefficients at surface pressure as a function of frequency for several mechanism.

Undersaturated Gas Sands" discussed the effects of various pore geometries on frequency attenuation in low saturation environments. They concluded that "The squirt flow induced by wave excitation results in viscous dissipation and wave attenuation". Further they stated that "At seismic and ultrasonic frequencies, the important mechanisms of wave attenuation in unconsolidated sand and porous rocks include friction between grain boundaries, bulk fluid (Biot) flow, and squirt flow (including viscous relaxation)". These mechanisms were found to occur in multiple lithologies, "Observations on sandstone, slate, limestone, and alundum (fused aluminum oxide) containing water 
show without exception a rapid increase of attenuation as saturation increases from 0 to 20 percent (summary by Johnston et al, 1979).”

"By assuming that each individual pore is in an undersaturated state (even those with smallest aspect ratio“, Mavko and Nur (1979) calculated the viscous dissipation as the pore liquid flowed in response to the squeezing of the pores by a passing wave. This squirt flow, and the accompanying attenuation, was "more pronounced in the flatter pores (cracks)." Figure 6, at left, which is Mavko and Nur's (1979) figure \#4, shows

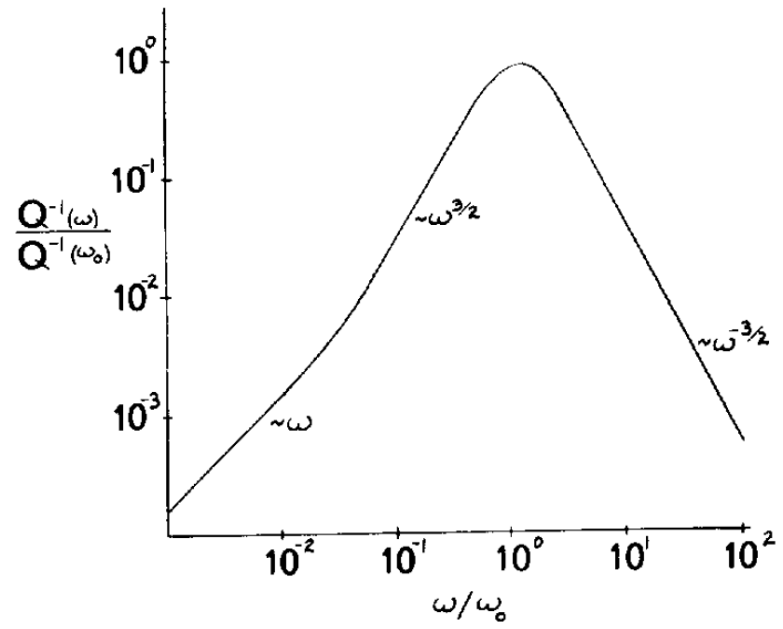

Figure 6: Mavko's figure 4 showing the frequency dependency of $Q^{-1}$ for the parallel walled pore in compression.

the results of their attenuation $\left(\mathrm{Q}^{-1}\right)$ is found to be extremely sensitive to the aspect ratios of the pores and the liquid droplets occupying the pores, with flatter pores and drops resulting in higher attenuation. This work is among the first to suggest that the shape of the permeability effects attenuation.

In another theoretical study, the results of which were published by White in 1975 , it was shown that a large amount of attenuation could result when a seismic wave passes through a region that contains macroscopic pockets of gas but was otherwise saturated. White's figure \#2,

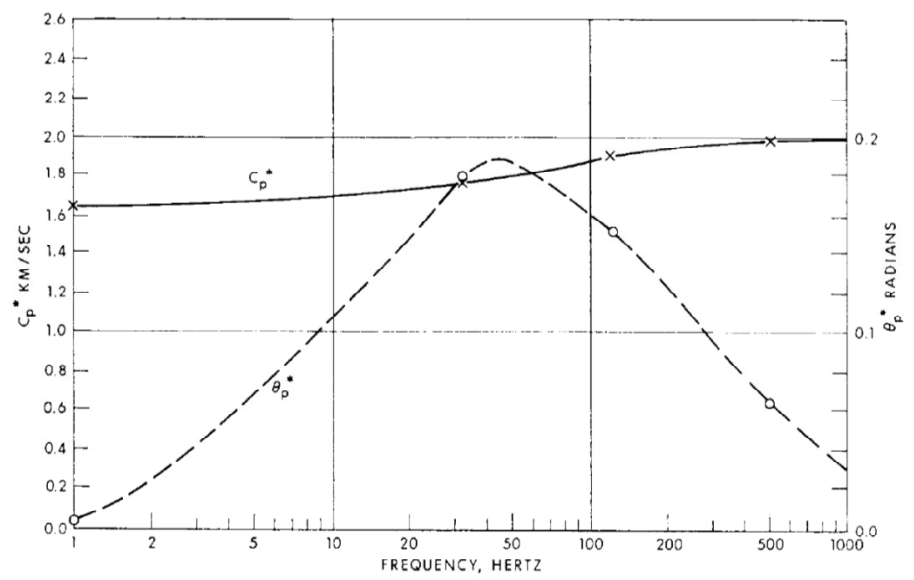

Figure 7: White's figure \#2 showing the relationship between frequency, phase angle and velocity.

shown here in this paper's Figure 7, shows the relationship between phase angle, velocity and frequency. 
K. W. Winkler in 1983 experimentally confirmed Palmer and Traviolia's observations that in an idealized system of glass beads frequency attenuation is negligible but in real rock environments, such as brine saturated sandstones, it does occur. Additionally, the attenuation was greater for higher frequencies. This trend was observed at all confining pressures, which are shown in Figure 8 for the Berea Sandstone at 2.5, 5, 20, and $40 \mathrm{MPa}$.
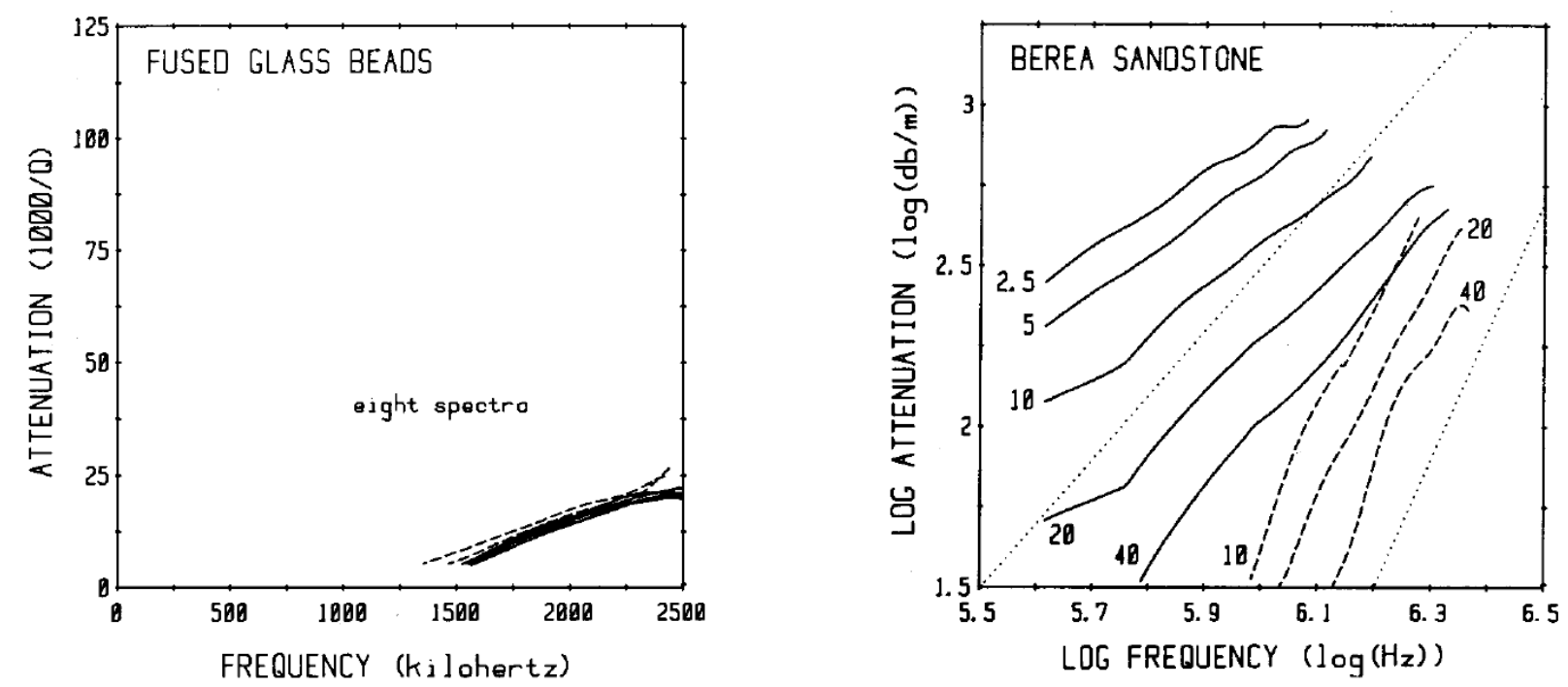

Figure 8: Illustrations from Winkler, 1983 showing that attenuation for dry (dashed) and brine saturated (solid) Fused Glass Beads (on left) and one of the formations test at various confining pressures.

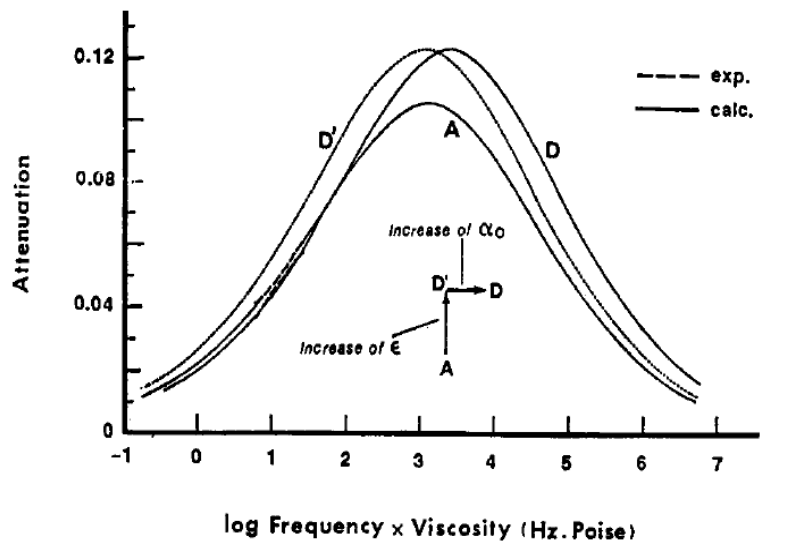

Figure 9: Shear wave attenuation versus $\log$ frequency $\mathbf{x}$ viscosity
Somerstein et al, (1984) investigated frequency attenuation in radio geotomography studies of an oil shale retort. They were able to show that frequency attenuation occurred in zones of high temperature and zones where "the penetration of moisture into the semifractured region" had occurred in a portion of the retort extending out from the rubble border. Nichols, 
Muir and Schoenberg (1989) discuss a method for modeling elastic properties of rocks and the effect of fracture distributions but without reference to the effects on frequency.

Vo-Thank, 1990 concluded from experiments that fluid viscosity was a main factor in the shear-wave attenuation and velocity in saturated sandstones. Attenuation was observed in glycerolsaturated sandstones related to squirt flow mechanisms. Figure 9 shows their calculations and experimental results.

An important observation into this phenomenon was made by J. Dvorkin and A. Nur in 1991. They stated that permeability "is related to attenuation through pore geometry". They concluded that in the case of linear fracture systems "a satisfactory agreement is found between predicted attenuation-permeability curves and experimental data obtained on sandstone samples of approx. constant porosity".

Dvorkin and Nur's paper in 1993 again related attenuation to permeability and showed that great attenuation occurs in higher frequencies. They introduced what they termed a unified Biot -
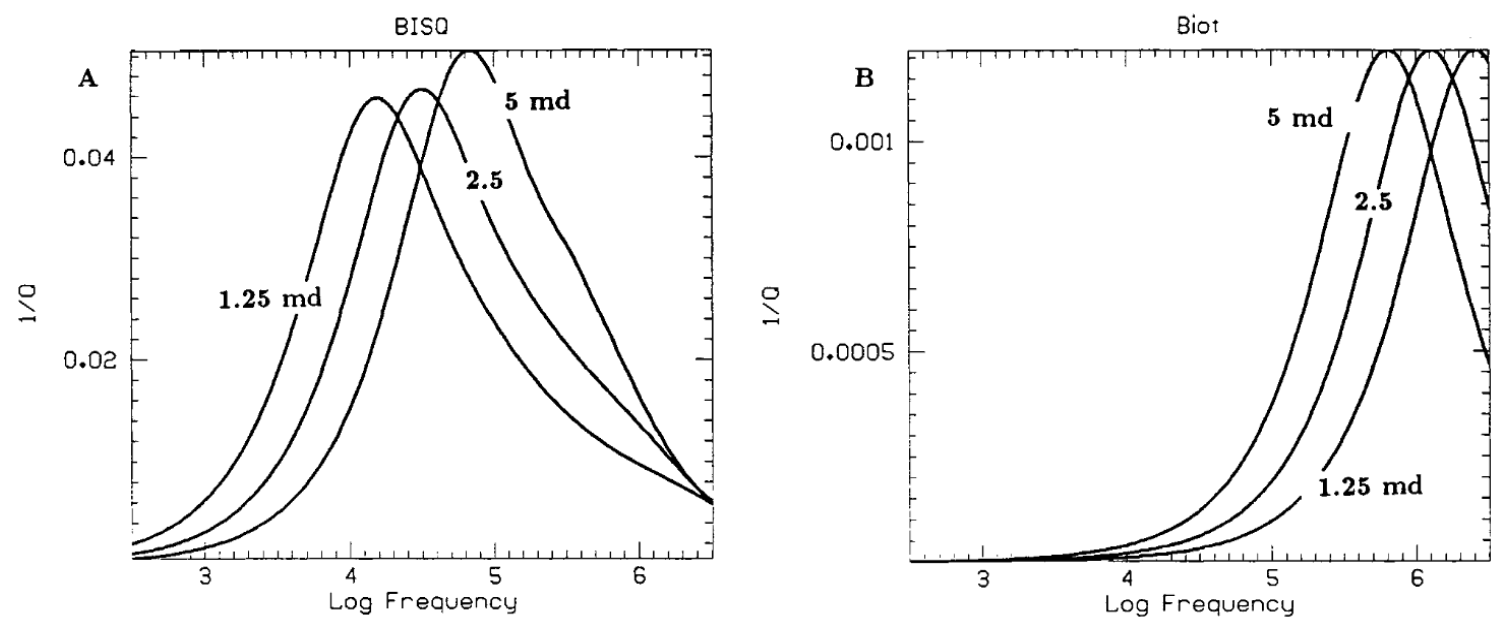

Figure 10: Q versus permeability for Dvorkin and Nur's unified dynamic poroelasticity model (Biot-Squirt flow) and their Biot only model.

Squirt flow model (BISQ). "The BISQ theory allows us to realistically calculate attenuation and velocity dispersion in saturated rocks as functions of such important parameters as frequency, fluid viscosity, fluid compressibility, porosity, and permeability." They suggested that an immediate 
application of the BISQ theory was in monitoring EOR processes where both the compressibility and the viscosity of pore fluids may change over time (Ito et al., 1979; Wang and Nur, 1990). Observations made during this study support the calculations for their Biot-Squirt flow model. Figure 10 shows that attenuation is greater at higher permeabilities and that attenuation is greater at higher frequencies.

Nabil Akbar, Jack Dvorkin and Amos Nur, in 1993 published more results from theoretical modeling and related attenuation to the direction of wave propagation. They conclude from their calculations, which were based on a theoretical model of a cylindrical pore filled with viscous fluid and embedded in an infinite isotropic elastic medium that "when a plane P-wave propagates perpendicular to the pore orientation attenuation is always higher than when a wave propagates parallel to this orientation." This work is among the first to recognize that the direction in which the wave encounters the porosity/permeability has effect on the amount

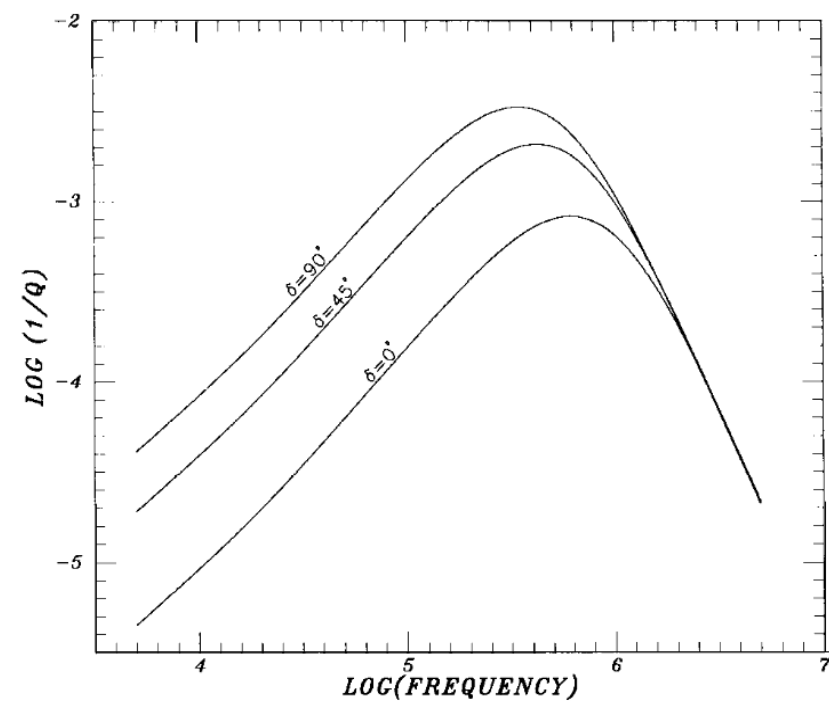

Figure 11: Attenuation versus frequency with regard to pore orientation.

of attenuation. Additionally, they concluded that attenuation of a low-frequency wave decreases with increasing permeability. This would result in lower frequencies being preserved in higher porosity / permeability zones.

Dilay and Eastwood in 1995 performed a 4D seismic analysis using spectral analysis of a steam flood and found that it showed attenuation of higher frequencies in higher porosity zones. They related this attenuation to the mixed phase gas / fluids in porosity. 
Batzle in 1996 concluded that in "a constant pore fluid type, permeability will control the motion and dissipation, thus making attenuation a permeability indicator." They also recognized that the amount of pressure in the pore space influenced the amount of attenuation. "Under vacuum dry conditions, little attenuation is observed, confirming the necessity for pore fluids to produce measurable attenuation. At $95 \%$ brine saturation at low pressure $(0.7$ attenuation becomes substantial and increases with frequency. At higher pressure $(8.6 \mathrm{Mpa})$, attenuation is decreased significantly although the frequency dependence is approximately constant."

Tsvankin in 1997 related Horizontal Transverse Isotropy (HTI) to parallel vertical penny-shaped cracks (fractures) that were contained within an isotropic medium. He found that these fractures affected the velocity of the passing wave. Velocity decreases along his "Symmetry-axis plane", which is shown in Figure 12.

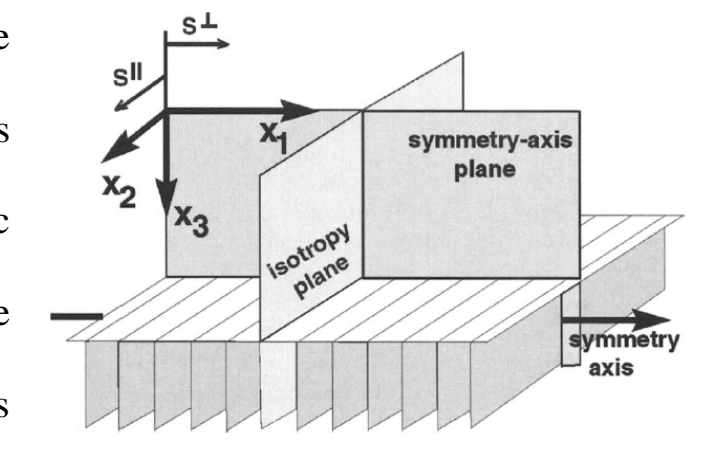
Endres and Knight in 1997 continued the Figure 12: Tsvankin's figure 1 showing his "Symmetry-axis" and "Isotropy" planes in a HTI system. investigations into the effects of fluid pressure communication and found that it was influenced by pore shape, size and orientation.

In 1998 Schoenberg found that large fractures effected low frequency waves but small fractures would not. They also found that very high frequency waves were strongly reflected by a fracture when the wavelength is large compared to the physical opening of the fracture.

Yang in 1998 investigated the influence of viscous coupling on the reflection and transmission at the boundary between saturated porous media and ordinary elastic media. He concluded that viscous coupling is strongly dependant on the hydraulic boundary condition (shape), frequency and the angle of incidence. This effect is negligible for impermeable interfaces, but for permeable interfaces it is noticeable. Additionally, frequency attenuation is not observable to any great degree for an impermeable interface but is for a permeable one. 
Pan in his 1999 thesis investigated methods to stimulate oil production through "Frequency Excitation." During his investigations he also found that the effective porosity variation representing solid motion in porous media is also a function of frequency.

Haugen and Schoenberg in 2000 discussed the results from modeling scattering attenuation due to the presence of fractures. This they relate to the compliance of the fracture with linear slip theory and showed that the reflectivity, and therefore the transmissivity, of a fracture is dependent on slowness along the fracture and on frequency. They also indicated that the "most useful information will be extracted from knowledge of amplitude as a function of incident angle (i.e., as a function of slowness along the fracture) and frequency."

Gurevich in 2002 mathematically investigated viscoelastic, poroelastic and scattering attenuation mechanisms. His results indicated that for low fluid viscosities poroelastic effects will dominate whereas for high fluid viscosities viscoelastic attenuation dominates. These may be of importance when analyzing reservoirs that have been flooded with $\mathrm{CO}_{2}$, a low viscosity fluid when injected at sufficient pressures.

Brajanovski reported in two papers published in 2003 significant differences in attenuation between dry and saturated pores and fractures. Greater attenuation (and effects on velocity) occurred in the liquid saturated materials. The characteristic frequency of the attenuation and dispersion depends on the background permeability, fluid viscosity, fracture density, and spacing. He indicates that "When both pores and fractures are dry, such material is equivalent to a transversely isotropic elastic porous material with linear-slip interfaces. When saturated with a liquid this material exhibits significant attenuation and velocity dispersion due to wave induced fluid flow between pores and fractures. At low frequencies the material properties are equal to those obtained by anisotropic Gassmann theory applied to a porous material with linear-slip interfaces. At high frequencies, the results are equivalent to those for fractures in a solid (nonporous) background." 
In his second paper during 2003 entitled "Attenuation and dispersion of compressional waves in porous rocks with aligned fractures", Brajanovski modeled fractures as very thin and high porous layers in a porous background. He found that when fractures were liquid saturated the system caused significant attenuation and velocity dispersion as a result of wave induced fluid flow between matrix pore space and the fractures. The characteristic frequency of this attenuation and dispersion depended upon the matrix permeability, fluid viscosity, the fracture density and spacing. His theoretical results agreed with his mathematical simulations using the reflectivity algorithm generalized to poroelasticity. His work also showed that fracture compliance (which he referred to as "fracture weakness") also caused significant attenuation and velocity dispersion. Figure 13 shows Brajanovski's figure 2 for a constant porosity system but for a fracture of varying weakness. This clearly shows increasing attenuation for more compliant fracture systems.

In 2003 Vlastos published work
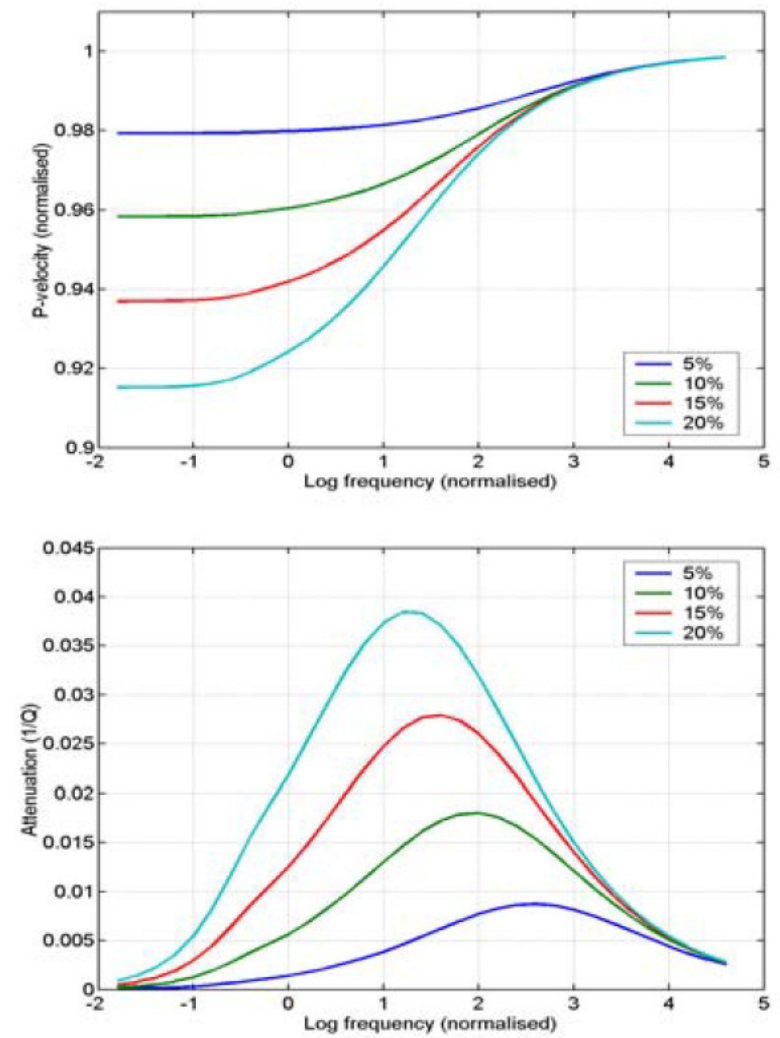

Figure 13: Brajanovski's figure 2 showing Pwave velocity dispersion and attenuation for a fracture system of varying strength in a fixed porosity.

showing that an increase in pore pressure causes

a shift of the energy towards lower frequencies. He went on to conclude also that effects on the wavefield by the pore fluid can vary significantly with the source-receiver direction.

Yamamoto in 2003 published work that showed the attenuation predicted by a super-k model for a carbonate was four times greater than that predicted by the Biot theory. The higher attenuation was related to pore fluid displacement. The enhanced loss mechanism is the reduction of the effective 
stiffness of pore water produced by the relaxed state of the squirt-flow mechanism. He concluded that the permeability imaging technique "can be applied to the attenuation and velocity data measured by other seismic methods."

In 2004 Kozlov concluded that when low-frequency waves propagate across a pore, the pore pressure has time to equilibrate due to fluid flow; however, this does not occur for moderately high frequencies.

Thompson, 2004 - Mark Chapman (Geophysical Prospecting, 2002) addresses fractures in porous media for two scales: microscopic pore/cracks and mesoscopic aligned fractures. The model predicts frequency-dependent anisotropy and forms the basis of fracture size estimation from seismic anisotropy.

Thompson (2004) noted that seismic waves are slowed and attenuated when they cross a fracture boundary. The fractures make the rock more compressible and compliant in the direction normal to the fractures. The effect appears to be more pronounced if the fracture is gas-filled or filled with a compressible fluid. A side product of attenuation is that the effect is also frequency-dependent, with higher frequencies preferentially attenuated across the fracture. On the microscopic-scale, current thinking is that the fractures weaken the rock for both $\mathrm{P}$ and $\mathrm{S}$ waves, absorbing the higher frequencies as the fluids 'slosh about' in the fracture pore space.

In 2004 Sharma discussed "hydraulic anisotropy" and concluded that it may lead to anisotropic propagation in an isotropic porous solid. Such an anisotropic propagation is small and restricted to high-frequency regime.

Batzle in 2006 discussed fluid mobility with respect to permeability and fluid viscosity and their effects on velocity and attenuation. He concluded that in low fluid mobility environments, such as shales or heavy oil reservoirs, pore-pressure remains unrelaxed at a wide range of frequencies, including seismic frequencies, and velocity will, therefore, be unaffected. However, in reservoirs 
with high fluid mobility, such as porous and permeable carbonates, attenuation and velocity dispersion can occur. These same relationships were also investigated by Carcione (2006).

In 2006 Dvorkin's investigations concluded that Biot and squirt flow attenuation mechanisms were not likely to occur at the pore scale for frequencies normally associated with seismic energies. However, viscoelastic attenuation could arise due to crossflow between fully liquid-saturated patches and the surrounding rock with partial gas saturation since these patches are larger than the regular pore spaces.

Schoenberg in 2006 discussed what he referred to as membrane permeability. He shows that this is the controlling factor in scattering amplitudes of slow $\mathrm{P}$ waves for very low-permeability fractures. He relates this to attenuation of these waves.

Recently (2007) Liu et al., published the results of a study that used the azimuthal information from VSP data. In this study they determined that amplitude attenuation does not coincide with velocity attenuation in fractured systems.

As mentioned in chapter 1 of this paper Muller (2010) provides a major review of studies published during the decades since Biot's original work. He organized these studies into three groupings; those based on Biot's poroelasticity theory, those based on viscoelasticity and those based on elastodynamic theory where local fluid flow is involved. He also indicates that in recent years some theoretical models have shown support for Biot's poroelasticity approach. He concludes that the application of these theoretical models to real seismic data required further studies. Muller's review is a significant synthesis of the current state of knowledge within this field. Many of these studies indicate that attenuation is expected in zones of higher matrix porosity /permeability while others deal with attenuation associated with fracture systems. Some indicate the directionality that should be expected with regard to fracture systems. These theoretical and mathematically based studies provide support for the field based studies described in this paper. 


\subsubsection{Previous Work - Spectral Decomposition}

Various methods of spectral decomposition have been discussed in the literature for approximately the past 20 years. As with the concept of frequency attenuation due to high porosity/permeability, discussed above, most of these works have been entirely theoretical.

Chakraborty (1995) - investigated three different methods of spectral decomposition during the analysis of synthetic seismograms and individual traces within shot point gathers from Sweden. These were the matching pursuit decomposition (MPD) method, the continuous wavelet transform (CWT) method and the short time Fourier transform (STFT) method. Chakraborty concluded that of these three methods, the MPD method provided the best localization in both time and frequency as can be
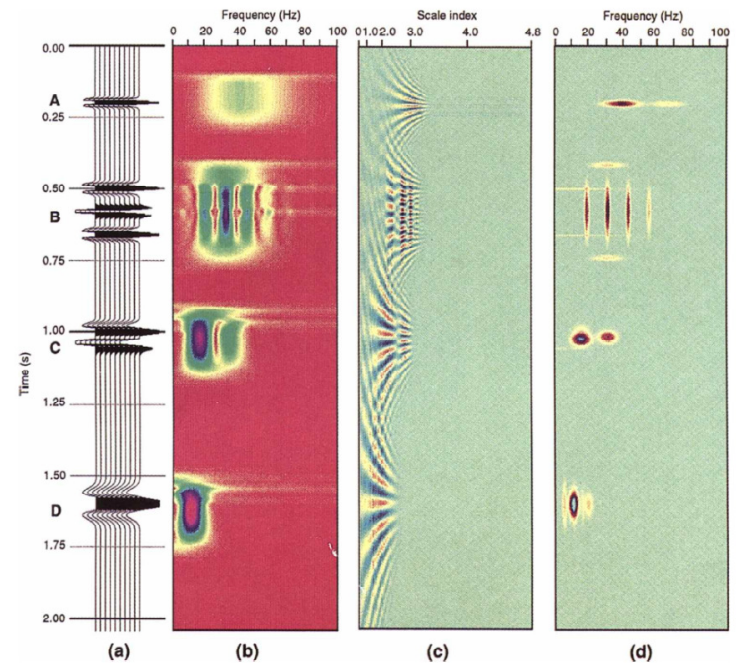

Figure 14: Chakraborty's figure 5 showing a comparison of the STFT (b), the CWT (c), and the MPD (d) methods.

seen in Figure 14.

James Gridley and Greg Partyka (1997) define Spectral Decomposition as the "separation of a time series into its amplitude and phase components via the Fourier transform" in their paper entitled "Processing and interpretational aspects of spectral decomposition". In the paper they demonstrate uses of this method during the interpretation of subtle stratigraphic features within a 3D volume from the Gulf of Mexico. They concluded that spectral decomposition was quite powerful for the interpretation and mapping of seismic features "spatial position, traveltime, and frequency amplitude and phase".

Partyka presented in 1999 essentially the same work as in his 1997 paper mentioned above.

Montoya in 2005 published the results of a study of a 3D seismic survey in the Gulf of Mexico using spectral decomposition. She used the technique to characterize various depositional 
environments including turbidite fans and distributary channel and channel-lobe complexes. This analysis was performed in the depth domain with wavenumber rather than frequency and showed that this analysis was significant for imaging different environments of deposition.

During 2006 Odebeatu, Chapman Zhang, Liu and Li presented at the EAGE's 68th annual meeting in Vienna and then later published in The Leading Edge case studies in which they demononstrated the analysis of iso-frequency volumes for AVO analysis. In their North Sea example they show a bright spot in $15 \mathrm{~Hz}$ data that is not observed in the $30 \mathrm{~Hz}$ in their Figure 1 (from The Leading Edge publication. They indicate that this effect was observed for other frequencies and that the reservoir was bright at low frequency, but not for higher frequencies. They related these amplitude and AVO anomalies to frequency- dependent phenomena related to gas saturation.

In another of their case studies, taken from the Ordos Basin in China, they demonstrate that difference mapping of the amplitudes between the $30 \mathrm{~Hz}$ and $15 \mathrm{~Hz}$ iso-frequency data illustrated the significantly stronger amplitudes of the lower frequency in a porous, gas filled reservoir. I recognized that this iso-frequency based analysis, which they employed for investigating the AVO response of gas filled reservoirs, could also be applied for identifying frequency attenuation caused by zones of high porosity.

Hall in his 2006 Leading Edge paper entitled "Predicting bed thickness with cepstral decomposition" described the use of a specialized derivation of spectral decomposition, Cepstral decomposition and quefrency analysis on a synthetic data to demonstrate how the technique could be used in the estimation of bed thickness.

Henderson in 2007 applies the use of visualization techniques (RGB) to spectral decomposed volumes for the purpose of geobody delineation. He describes a workflow designed to use various RGB visualization and opacity settings to evaluate which frequency volumes contain useful information. This paper is the first to describe using this technique to evaluate spectral decomposition volumes. 
Zeng's 2007 paper discusses the use of stratal slices through spectral decomposition volumes for seismic geomorphology analysis. Similar to Gridley, Partyka and Montoya's previous studies for geobody analysis, Zeng's paper describes the classification of a meandering fluvial system in the Gulf of Mexico using stratal slicing through spectrally decomposed volumes.

Nearly all of these studies were performed on seismic data sets from the Gulf of Mexico, where rock velocities are slower than in other, more compacted and mature areas. Henderson's 2007 workflow paper is apparently based on the examination of a number of seismic volumes, however, their location is not discussed. Chakraborty's 1995 paper was based shot point gathers from Sweden and not 3D volumes. None of these studies discuss the use of spectral decomposition for the identification of zones of attenuation.

\subsubsection{Previous Work - Seismic Anisotropy}

It has been recognized for some time that various rock units transmit compressional energy at speeds that vary in different directions. These have been designated as VTI environments (Vertical Transverse Isotropy - where the vertical rock velocity varies from the horizontal rock velocity in all directions) and HTI environments (Horizontal Tranverse Isotropy - where the rock velocity varies in all directions vertically and horizontally). Early recognition of the HTI environment was published by McCollum and Snell in 1932 based on work they had performed on shale obtained from outcrop.

In 1955 Dix published what is now recognized as a major work on the estimation of rock velocity using seismic data. Levin in 1978 investigated the variation of p-wave velocity in an anisotropic medium and in his 1979 paper also discussed SH-wave and SV-wave variations. Seriff in 1991 confirmed Levin's ray tracing results. Sena in 1991 showed that using p-wave velocity data an estimate of the orientation of the horizontal axis of symmetry for a given layer at depth could be obtained.

Leon Thomsen, in his 1986 publication "Weak Elastic Anisotropy", presented new parameterization and equations related to the level of anisotropy generally of concern to exploration 
geophysicists. Sayers and Kachanov in their 1991 paper "A Simple Technique for Finding Effective Elastic Constants of Cracked Solids for Arbitary Crack Orientations Statistics" describe a method for evaluating the elastic stiffness tensor for fractured (cracked) formations. In their 1995 paper they use fracture (crack) density tensors to determine the amount of elastic anisotropy in brittle rocks as measured at ultrasonic frequencies.

The vast majority of the work in this field has dealt the detection and analysis of anisotropy in subsurface formations using velocity analysis. During roughly the past decade some studies have attempted to apply these theoretical and experimental findings to field data. Lynn in 1999 published two papers that dealt with the investigation of velocity anisotropy in HTI environments for two different fields in the Rocky Mountains. In both studies she demonstrated that horizontal velocity variation existed within these fields due to the presence of open natural fracture systems. In 2004 Lynn summarized various azimuthal velocity techniques that can be used for fracture detection. 


\subsection{Objectives and Scope}

The objective of my research was to investigate the potential use of iso-frequency volumes extracted from variable azimuth seismic volumes (azimuthal iso-frequency volumes) in order to interpret the location of zones of drained, high flow systems within a carbonate reservoir and characterize these zones according to their geometries.

To achieve this objective, iso-frequency volumes were derived through spectral decomposition and then the spectrally decomposed data were examined to identify zones of increased low frequency content. Based on correlations I made during the portion of this research performed for the Department of Energy (2009) I demonstrate a relationship between these zones of low frequency and drained, high flow zones within the matrix of the reservoir. Based on the significant amount of theoretical research, which I have summarized in the previous section of this chapter, strong low frequencies that are associated with drained, high flow systems should be due to the attenuation of higher frequencies in the seismic signal. The attenuation of the higher frequency component of the seismic spectrum would leave the lower frequency component to dominate. I interpreted azimuthal iso-frequency volumes in order to characterize porosity type and distribution

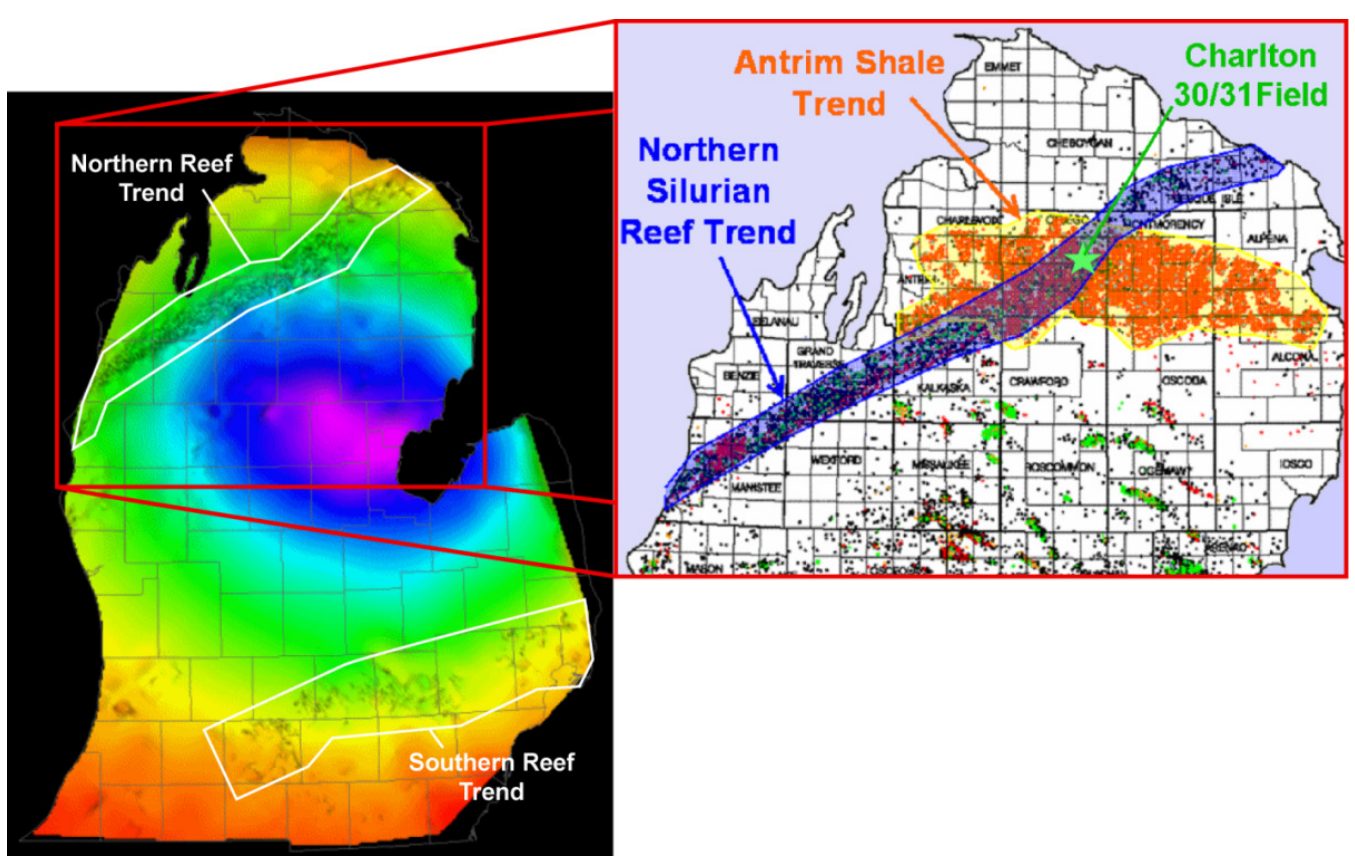

Figure 15: Location of the study area, the Charlton 30/31 Field in Northern Michigan Silurian Reef Trend. The structure map shown is on the top of the Silurian Niagaran Brown formation, which contains the Guelph formation reefs. 
within a carbonate reservoir, the Charlton 30/31 Silurian age reef in the Northern Michigan Basin (see Figure 15 below). I tested this theory using well data and 4D (or time lapse 3D) compressional (P-wave) seismic data acquired over this field during the Department of Energy research project. The DOE project was originally designed to monitor a $\mathrm{CO}_{2}$ flood undertaken by Core Energy to enhanced oil recovery from the reservoir.

Azimuthal seismic volumes developed from the baseline 3D seismic survey were used to create the azimuthal iso-frequency volumes. My examination of these iso-frequency volumes revealed zones of dominant low frequencies which I showed were coincident with either high matrix porosity or the presence of open, natural fractures. The zones observed at the same location in all azimuthal volumes indicated that the frequency attenuation at that location was isotropic in nature and attributable to uniform distribution of high matrix porosity. Figure 16 provides a simplified illustration of a high matrix porosity feature, such as a zone containing vuggy porosity that would not lead to wavefield polarization but would produce attenuation in all directions.

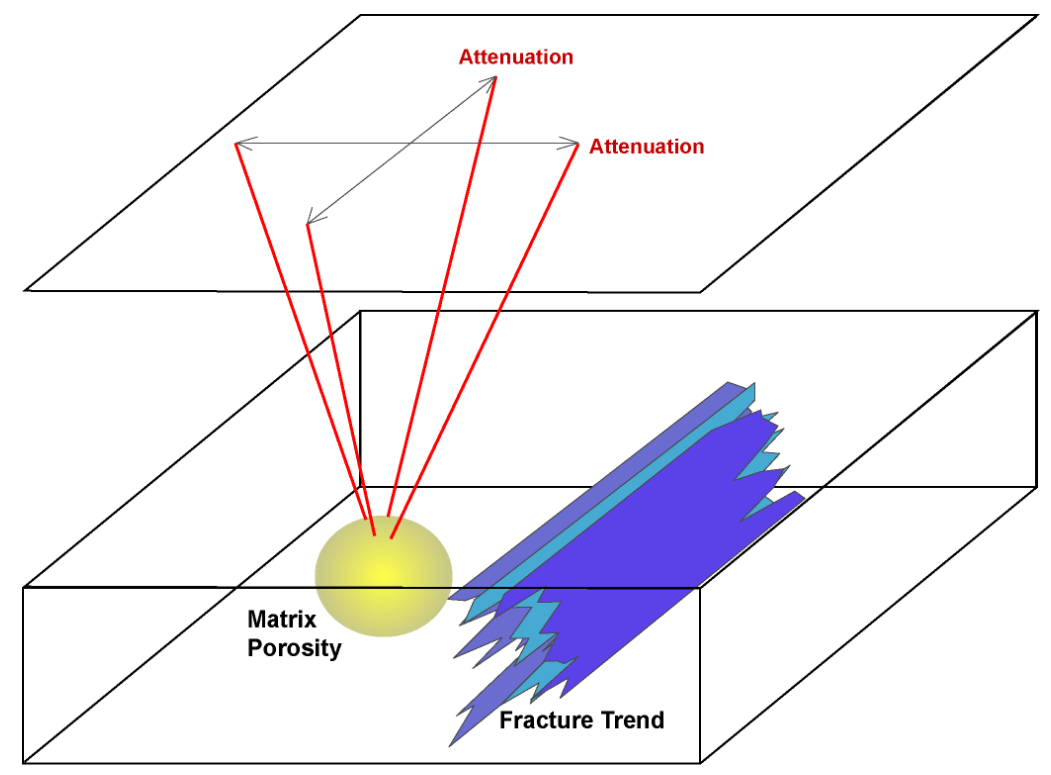

Figure 16: Isotropic frequency attenuation as a result of a drained, high flow system within the matrix of the reservoir. 
Zones of attenuation occurring only on certain azimuthal volumes indicate that the frequency content at that point in the reservoir is anisotropic in nature. These were interpreted as zones of open, natural fractures. In this later case the directionality allowed the orientation of the fracture trends to be determined. Figure 17 illustrates this case. Note that an elongate zone of high porosity might have a similar influence on frequency distribution since CMP reflection events occurring along the axis of higher porosity (inferred lower velocity zone) may have increased travel path length within the feature depending on its width and length. For example, along the axis of the feature, its dimension might be much larger than the Fresnel zone radius, while in the direction across the axis of the feature its influence may be restricted in size to a region much smaller than the Fresnel zone radius.

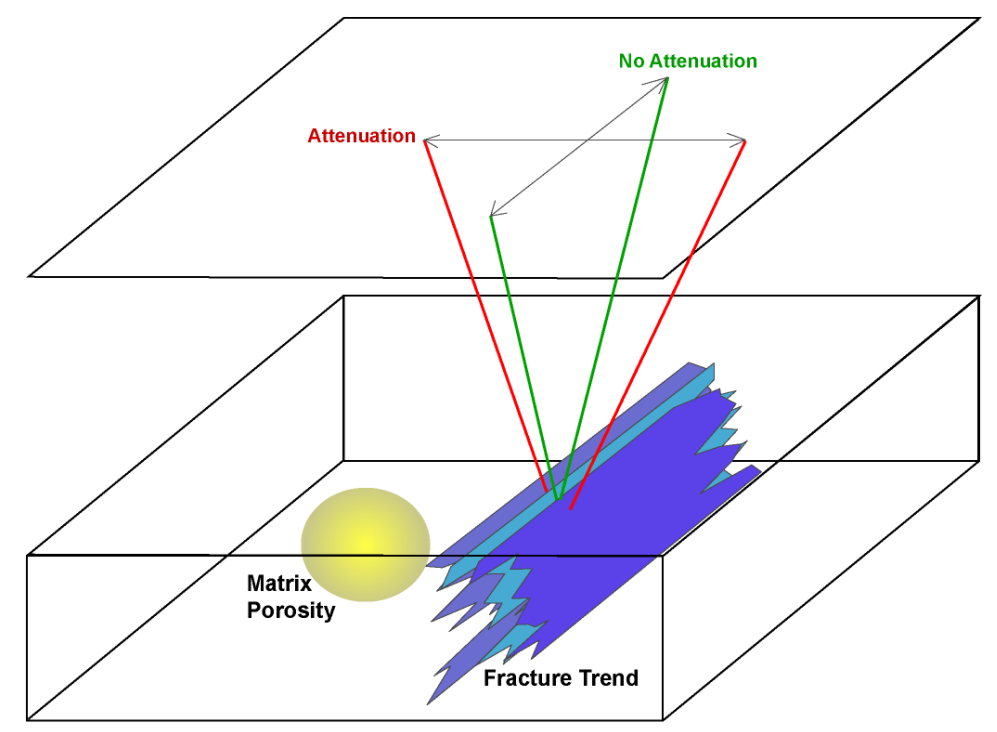

Figure 17: Anisotropic frequency attenuation as a result of a linear, drained, high flow system resulting from open, natural fractures.

Using this interpretation methodology the porosity distribution within the Charlton 30/31 Reef was interpreted. This porosity distribution was tested in two locations by the drilling of two new wells within the reef after the interpretation was performed. Additionally, a reservoir simulation developed on this porosity distribution was successful in matching 25 years of production history. 
Additional validations of this method were performed during later stages of this research and are reported in later chapters. 


\section{CHAPTER 3 - DESCRIPTION OF PREVIOUS WORK}

\subsection{Introduction}

\subsubsection{The Northern Silurian Reef Trend of the Michigan Basin}

Within the Michigan Basin two well-defined reef trends exist, one in the northern portion of the basin and the other in the southern, shown previously in Figure 15. These are pinnacle reefs of Silurian age (Niagaran) and occur in the Guelph Formation (formally known as the Niagaran Brown). Although first discovered in 1885 (Shaver, 1977), the majority of these pinnacle reefs were not located until the 1970s and 1980s. 2D seismic data proved to be instrumental in locating individual reefs within the trends. To date, over 1100 reefs have been identified within the northern and southern trends. These range in depth from 915 to 2135 meters. These reefs average in height 107 meters, however, some reach up to 210 meters high. These taller reefs are located in the northern trend (Figure 15). The diameters of these reefs are highly variable, with smaller diameter (but taller) reefs in the north and shorter, more broadly developed reefs found in the southern trend. The aerial distribution of reefs within the northern trend typically ranges between 40 and 150 acres, however, as typical with most oil reservoirs, some achieve larger sizes. The Charlton 30/31 oil field (location shown in Figure 15) is approximately 300 acres in size.

Although these reservoirs are all carbonate in nature they do exhibit some variation in lithology. This ranges from completely dolomitized to mixed limestone/dolomite reservoirs which have a low porosity, low permeability limestone matrix with irregularly dolomitized intervals to entirely limestone throughout the reservoir interval. These dolomitized zones provide a network of higher porosity / permeability which controls fluid flow throughout the reservoir. Gravity segregation/gravity drainage effects are apparent as a depletion mechanism in many of these fields.

Methane gas production occurs from the stratigraphically shallower Antrim shale formation. The aerial extent of this formation at the onset of this study is also shown in Figure 15. Since that time development of this trend has continued, mainly to the west. $\mathrm{CO}_{2}$ is produced as an associated 
byproduct from this production. The Antrim is a desorption-controlled reservoir and produces with a slowly increasing $\mathrm{CO}_{2}$ cut. During processing the $\mathrm{CO}_{2}$ is removed in order to meet pipeline quality specifications. The vast majority of the excess $\mathrm{CO}_{2}$ is rejected to the atmosphere. These two producing trends, the Guelph and the Antrim shale coexist in the area of the Northern Reef Trend shown in Figure 15.

\subsection{Dept. of Energy Project Description}

I performed a number of geophysical analyses on the Baseline seismic survey during the research I conducted for the Department of Energy. I conducted wavelet analysis in a number of locations within the volume in the vicinity of wells that had sonic logs. Since the reflectivity series was known at these well locations, wavelets were estimated using a deterministic wavelet extraction procedure. These extracted wavelets were combined with the well log data to produce good quality well-to-seismic ties. Figure 18 shows the resulting well-to-seismic tie obtained for the Charlton 1-30 well, which is the most northern well in the field. This well was used during the project as the enhanced oil recovery producer. Additional wavelet analysis and well to seismic ties were performed at other locations within the baseline seismic survey. These will be discussed in greater detail later in this chapter under "Initial Investigations".

Well-to-seismic ties confirmed the location of the reservoir within the seismic volume and established a time to depth relationship. Following this task seismic horizon interpretation was performed through the 3D volume. Seismic time maps were developed for each of the major horizons within the seismic survey. Velocity maps were also developed based on the time to depth relationships established with the well-to-seismic ties. Next the time and velocity maps were combined to produce depth maps for the top and base of the reservoir and the overlaying A1 Carbonate, see Figure 18. 


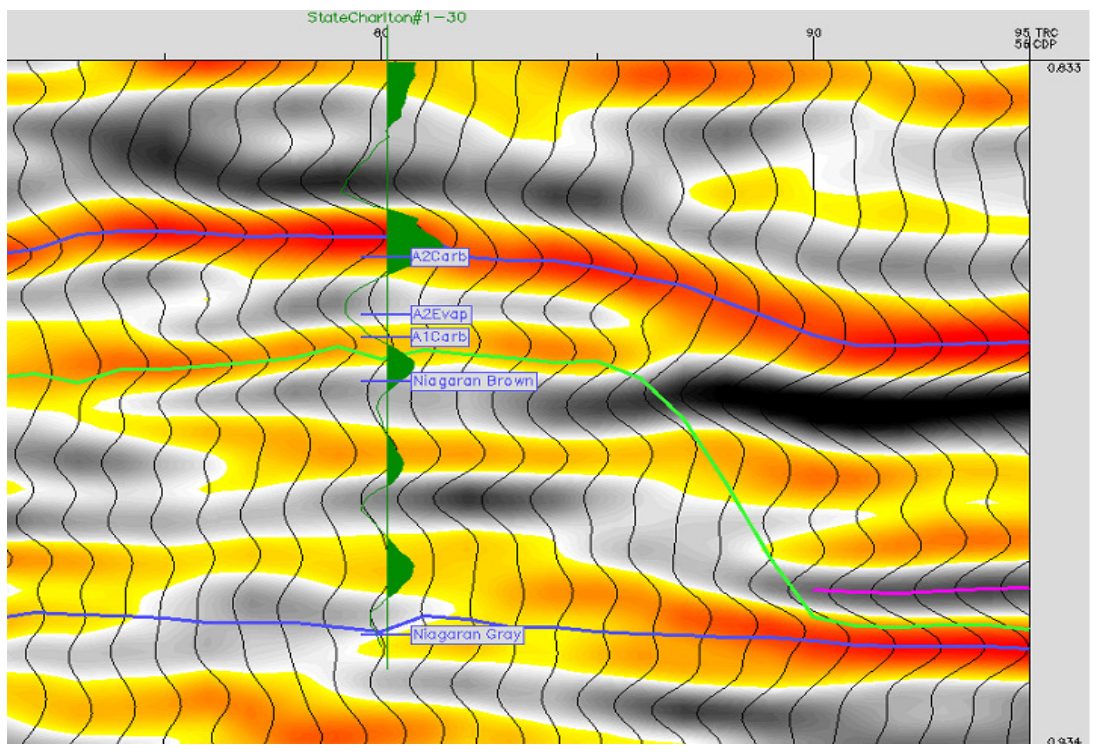

Figure 18: Well-to-seismic tie for the State Charlton \#1-30 borehole. The synthetic (in green) matches the seismic quite well and is considered to be of very good to excellent quality.

Various seismic attributes were extracted from the Baseline 3-D volume. The well-to-seismic ties allowed a well constrained time to depth relationship to be established and I was then able to compare instantaneous frequency and reflection magnitude seismic attributes to the porosity measurements obtained with the well log data. I accomplished this by using time slices through the seismic volume at $2 \mathrm{~ms}$ intervals. These time slices were converted to depth in order to allow the comparison with well log data. Log porosity values were averaged through the interval bounded by seismic slice data. This average log value within this window was then compared to the seismic attribute value at the center of that same window. The comparison of the average log porosity values with the seismic attributes revealed a relationship that could be used to map high porosity values between the wells using the seismic data. The details of this relationship are discussed in Chapter 4, "Frequency Attenuation and Its Relationship to Drained, High Flow Systems ".

With the porosity volume established for the reservoir using well and seismic data a "static model" (also referred to as a "geologic model" within the oil and gas industry) was constructed for the reservoir using the Petrel modeling software package. The term "static model" is often used as it pertains to a model of a reservoir's properties that do not typically change during the production life 
of the field. These generally include characteristics such as lithology, porosity and permeability. This static model was then used as the initial input into the field's reservoir simulation, also known as a "dynamic model". The term "dynamic model" is often used in reservoir engineering to indicate a model of reservoir characteristics that can change (and is, therefore, dynamic) during the field's production cycle. These characteristics include pressure, as well as the percentages of oil, gas and water within the reservoirs pore space.

A reservoir simulation of this field was then performed using this dynamic model. The simulation's initial state was set using reservoir characteristics established at the time of the field's discovery in 1974. The reservoir simulation was then brought forward at regular time steps (intervals) until the field's abandonment in 1997, with the field's development wells being added to the model at the time they were actually drilled. The simulation estimated the distribution of various reservoir constituents (amounts of oil, gas and water) within the reservoir at these regular time steps. It also estimated the production that should have been obtained by each of the field's wellbores.

These estimates of oil, gas and water production at the various well locations within the field were then compared at the regular time intervals with the recorded production figures that were obtained by the actual wells. This comparison is referred to in the industry as "production history matching". A good quality production history match can only occur if the distribution of the reservoir's static and dynamic properties were modeled accurately. A poor quality production history match indicates problems with the static and/or dynamic models used for the simulation.

The reservoir simulation performed using the final reservoir static model was judged to have a good quality production history match of the Charlton 30/31 field's primary production history, although reservoir engineering analysis indicated that some minor adjustment was initially needed between the injector and EOR producer. Additionally, well work-overs indicated that a water "dump flood" had occurred between the time primary production had ceased in 1997 and the beginning of the well workovers in 2004. Both of these will be discussed later in this thesis. 
The good history matched simulation validated the distribution of porosity and permeability within the Charlton 30/31 reservoir and, therefore, the seismic attribute analysis and methodology used to determine the porosity distribution.

The production history match to production from 1974 to 1997 obtained through simulation indicates that the reservoir model developed in this study provided a valid representation of reservoir properties. Using this model as a starting point, a number of new, predictive, forward-looking reservoir simulations were made in order to estimate where, within the reservoir, injected $\mathrm{CO}_{2}$ would move. These predictive simulations were made based on varying injection rates, sweep strategies and various $\mathrm{CO}_{2}$ injector and EOR producer configurations. The goal of these simulations was to identify the most effective methodology that maximized the recovery of oil. All of these predictive simulations were made prior to the acquisition of the second 3D survey.

A decision was made by the operator, Core Energy, to inject $\mathrm{CO}_{2}$ into the basal reservoir strata using the State Charlton "C" 2-30 borehole, which was the only deviated borehole within the field, see Figure 19. Additionally, the State Charlton 1-30 borehole was selected for enhanced oil recovery well. $\mathrm{CO}_{2}$ injection commenced in August of 2005 and was maintained at varying rates until September 2006 with first oil being produced in the State Charlton 1-30 borehole in June 2006.

A second (or "Monitor") 3D seismic survey was acquired during September 2007 over most of the field and duplicated the first (or "Baseline") survey, as much as possible. However, as the simulation and new well data available at that time indicated that the $\mathrm{CO}_{2}$ was concentrated in the northern portion of the field, the second seismic survey was not acquired over the extreme southern end of the area covered by the Baseline 3D survey. Basic processing was performed on the second 3D seismic survey and, finally, 4D processing methods were applied to both the Baseline and the Monitor surveys by WesternGeco to produce two 3D volumes that could be compared. This final 
processing produced the $3 \mathrm{D}$ time-lapse data set needed to detect changes in the reservoir that might have been brought about by the injection of the $\mathrm{CO}_{2}$. This will be discussed in the next chapter.

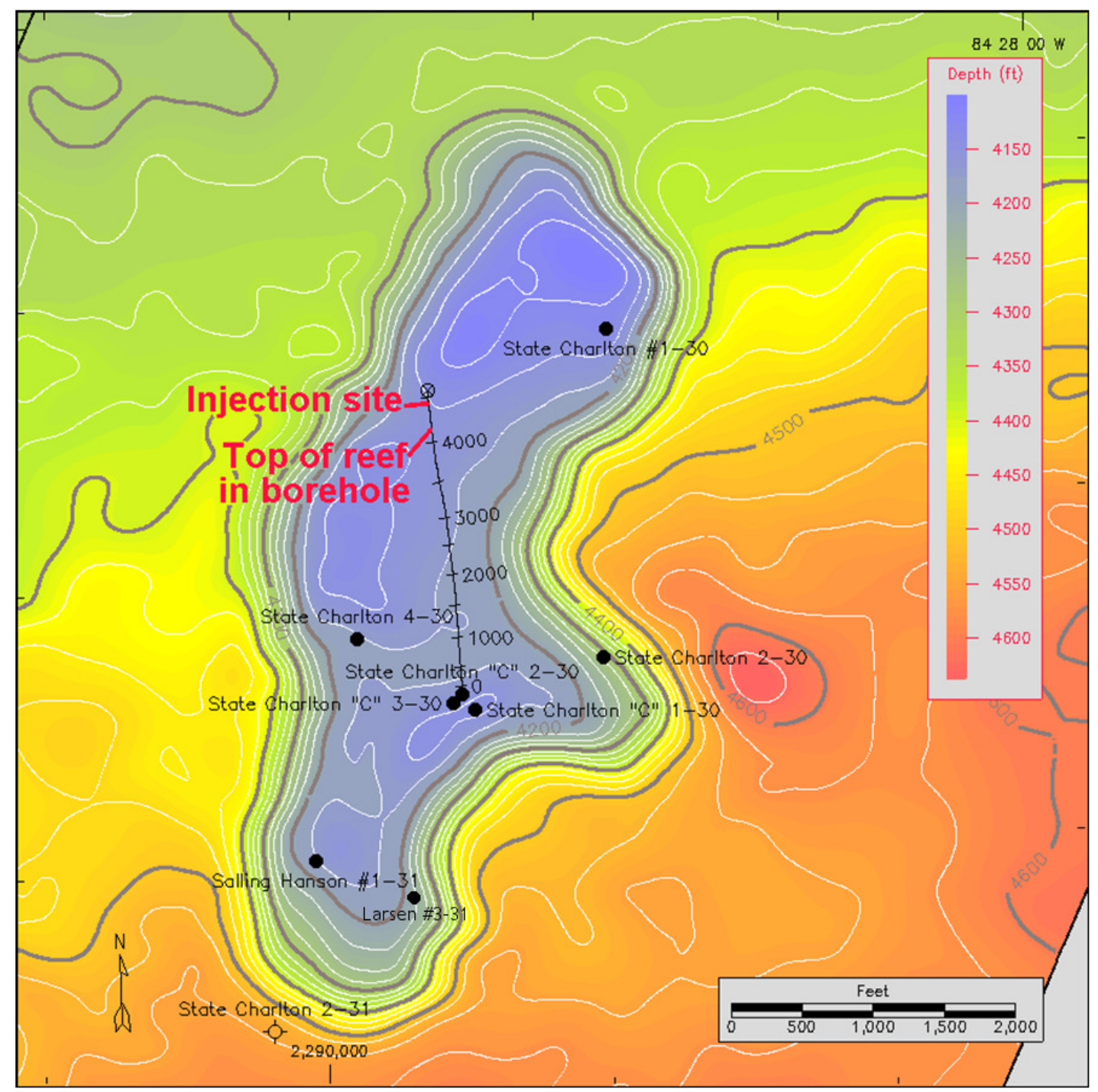

Figure 19: Structural map of the Charlton 30/31 field on the top of the Guelph Formation.

Interpretation of the 4D seismic data indicated that a significant amplitude change, not attributable to differences in acquisition or processing, existed at the locations within the reef predicted by the reservoir simulation. The reservoir simulation discussed above was based on the porosity distribution obtained from seismic attributes calculated using the Baseline 3D survey. Using this validated reservoir simulation the location of remaining oil within the reef at the time the Monitor survey was acquired was estimated. Based on this analysis recommendations were made 
concerning the drilling of additional EOR wells. Additionally, the economic impact of this project has been estimated in terms of both enhanced oil recovery and $\mathrm{CO}_{2}$ sequestration potential. In the northern Michigan Basin alone, the Niagaran reef play is comprised of over 700 Niagaran reefs with reservoirs already depleted by primary production. Potentially there is over 1 billion bbls of oil (original oil in place minus primary recovery) remains in the reefs in Michigan (reference), much of which could be more efficiently mobilized utilizing techniques similar to those employed in this study. This is discussed in the Economic Impact section of my final report to the DOE, Toelle (2009) page 63.

\subsection{Data Description}

\subsubsection{Previously Existing Well Data}

The Charlton 30/31 oil field was discovered in 1972 with the drilling of the Salling Hanson \#1-31 well (API number 29073, Otsego Co., Michigan) in the southern part of the field. This discovery was made by the Shell Oil Company. A number of well logs, which were considered standard for the day, were run on this well. These included a sonic log as well as gamma ray and density logs. Shell next drilled the State Charlton \#1-30 well (API number 29989) during 1974 in the northern end of the field. Again a suite of logs was on during evaluation of this well. During 1975 the Amoco Production Company drilled the State Charlton \#2-30 (API number 30203), which is located on the eastern side of the field. This well did not penetrate the top of the reef, like the wells drilled prior to this, but instead penetrated the reef on the flank. This resulted in a shortened section of the reef being logged. This affected the well-to-seismic tie at this location, as the sides of the reef are very steep and not readily observable. Additionally during this year Amoco drilled the State Charlton "C" \#1-30 well (API number 30195) located at the center of the structure. This well encountered mechanical problems and was not productive. The Amoco State Charlton "C" \#3-30 well (API number 32605) was drilled during 1978 in order to replace the failed State Charlton "C" \#1-30 well.

The State Charlton "C" \#2-30 well has a surface location near the "C" \#1-30 and "C" \#3-30 wells but 
was drilled deviated so the it's borehole entered the reef significantly to the northwest of the surface location, (see Figure \#19).

These wells were primarily located and drilled based on 2-D seismic lines that were acquired over the field during the 1970s. This 2-D data set was not examined during the Department of Energy study due to its vintage and the fact that modern 3-D data have been acquired with the acquisition of the baseline 3-D survey.

\subsubsection{Baseline 3D Seismic Survey}

The "Baseline" 3D survey was obtained in March of 2004 and covered 2.5 square miles. This survey was acquired by Great Lakes Geophysical. The resulting bin spacing was 82.5 by 82.5 feet. Five pounds of dynamite supplied the energy source and the resulting processed volume had a

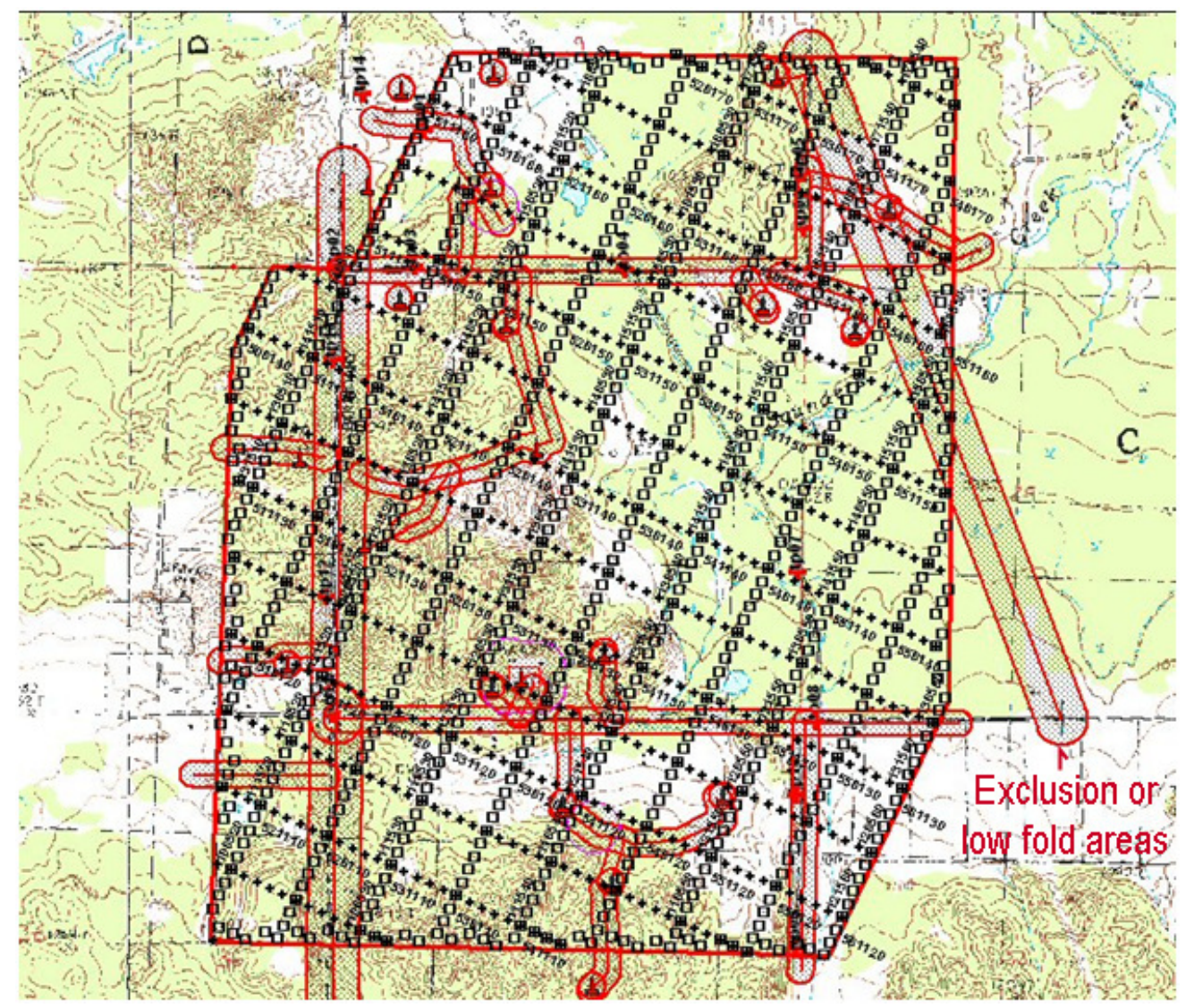

Figure 20: Acquisition exclusion zones for the Baseline 3D seismic survey. Exclusions or low fold zones are highlighted in red. 
sample rate of 1 millisecond. During the acquisition a number of acquisition exclusion zones were encountered. These are not uncommon when acquiring seismic data and are generally compensated for by adding shot/receiver pairs in other locations and through the processing of the data. These exclusion zones are shown in red outlines in Figure 20.

Once the acquisition of the Baseline 3D seismic survey was completed, the raw seismic data was processed by two different processing companies, WesternGeco and Sterling Seismic. This was done to insure that any data artifacts that may have resulted from a particular company's processing sequence would be noted and resolved prior to the interpretation of the volume. Processing induced artifacts such as these might have resulted in false positives being interpreted when examining the 4D survey and incorrectly relating these to movement of the $\mathrm{CO}_{2}$ within the reef.

The general processing sequence preformed on the Baseline 3D survey was as follows;

1. Reformat SEGD to Internal Format

2. Spherical Divergence Correction

3. Trace Edits

4. Refraction Analysis

5. 3D Geometry QC and Correction of Positioning of Source/Receiver

6. Elevation/Refraction Static Application

7. 3D Surface Consistent Deconvolution

8. Zero Phase Spectral Whitening

9. 3D CDP Sort

10. Velocity Analysis

11. 3D Surface Consistent Auto Statics

12. Velocity Analysis

13. 3D Surface Consistent Auto Statics

14. Noise Reduction as necessary 
15. CDP Statics

16. 3D CDP Stack

17. Post Stack Filter/Scaling/Decon or Whitening Application

18. Post Stack 3D Signal Enhancement -FXY Prediction Filter

19. Final Structural output

20. Post Stack 3D Finite Difference Migration

21. Post Migration 3D Signal Enhancement - FXY Prediction Filter (if necessary)

22. Migration output to SEGY

23. Targeted PSTM to Residual Velocity Field

24. Kirchoff Pre-Stack Time Migration (PSTM)

25. Residual Velocites

26. Pre-Stack Time Migration Stack output to SEGY

This 3-D seismic survey became the "Baseline" survey for the 4D seismic data set. A number of seismic interpretation methods were employed on this data set in order to develop an understanding of the reservoir. These included wavelet and frequency analyses as part of the well to seismic ties and are detailed later in this chapter.

\subsubsection{Azimuthal 3-D Seismic Surveys}

In addition to the basic processing described above four azimuthal seismic volumes were developed from the baseline 3-D survey. These volumes were developed using a $45^{\circ}$ angular swath during the binning of the CMP's. Additionally, the central azimuth for these angular swaths were $45^{\circ}$ to one another. The resulting configuration utilized all of the available data but did not allow for an overlap between the angular swaths. The four azimuths on which these volumes were developed were:

- $25^{\circ}$ (which included shot-receiver pairs between $2.5^{\circ}$ and $47.5^{\circ}$ and the reciprocal directions of $182.5^{\circ}$ and $227.5^{\circ}$, central azimuth of $205^{\circ}$ ) 
- $70^{\circ}$ (which included shot-receiver pairs between $47.5^{\circ}$ and $92.5^{\circ}$ and the reciprocal directions of $227.5^{\circ}$ and $272.5^{\circ}$, central azimuth of $250^{\circ}$ )

- $115^{\circ}$ (which included shot-receiver pairs between $92.5^{\circ}$ and $137.5^{\circ}$ and the reciprocal directions of $272.5^{\circ}$ and $317.5^{\circ}$, central azimuth of $295^{\circ}$ )

- $160^{\circ}$ (which included shot-receiver pairs between $137.5^{\circ}$ and $182.5^{\circ}$ and the reciprocal directions of $317.5^{\circ}$ and $2.5^{\circ}$, central azimuth of $340^{\circ}$ )

\subsubsection{Monitor 3D Seismic Survey}

The Monitor survey was acquired using the same acquisition company, Great Lakes

Geophysical, and parameters that were used in the acquisition of the Baseline 3-D survey. Unfortunately, due to the timing of the project it was not possible to acquire the Monitor survey at the same time of year as the Baseline 3-D survey. The Monitor 3-D seismic survey was acquired in September 2007 at the end of the summer and beginning of the fall, whereas, the Baseline survey was acquired in March at the end of the winter and beginning of the spring.

This difference in the time of year when the acquisitions occurred may have had some effect on the two data sets due to the water saturation of the surface formations, however, the normalization of the two data sets that occurred during the 4D processing would have adjusted for this. The study area is extensively covered with significant amount of glacial drift. At the end of the winter/beginning of spring these materials become saturated with snowmelt water. At the end of the summer/beginning of fall this water would not be present to the same degree. The presence or absence of water in these materials would have some effect on the seismic signal. This variation of signal was adjusted for during time-lapse processing of the two surveys. Although processing of the data enhanced the lost signal due to the effects of glacial drift saturation, it still must be taken into account.

The 4D processing sequence that was applied to the data set by WesternGeco is shown here;

- Survey/Seismic Data Merge 
- Resample

- Refraction Tomography

- Time Function Gain

- F-X Coherent Noise Suppression (FXCNS

- Swell Noise Attenuation (SWATT)

- Surface Consistent Deconvolution

- Deconvolution Operator Design

- Window Specification

- Surface Consistent Decomposition

- Time Variant Spectral Whitening

- Residual Statics (Miser 1)

- Preliminary Velocity Analysis

- Residual Statics (Miser 2)

- Preliminary Velocity Analysis

- Surface-Consistent Amplitude Compensation (SCAC)

- Residual Amplitude Analysis/Compensation (RAAC)

- Swell Noise Attenuation (SWATT)

- Surface-Consistent Amplitude Compensation (SCAC)

- 4D Data Matching

- Pre Migration Filter

- Kirchhoff Pre-Stack Time Migration

- Velocity Analysis

- Final Velocity Analysis

- NMO Compensation 
- Outer Trace Mute

- Stack

- Datum Correction

A comparison of the two surveys that make up the 4D seismic survey reveals that the two volumes are fairly similar. Figure 21 shows a comparison of in-line 1060 from both surveys. While subtle differences can be seen important characteristics, such as the amplitude variability along the flattened A2 Carbonate (0 time line), is fairly consistent. This comparison is discussed in greater detail in the next

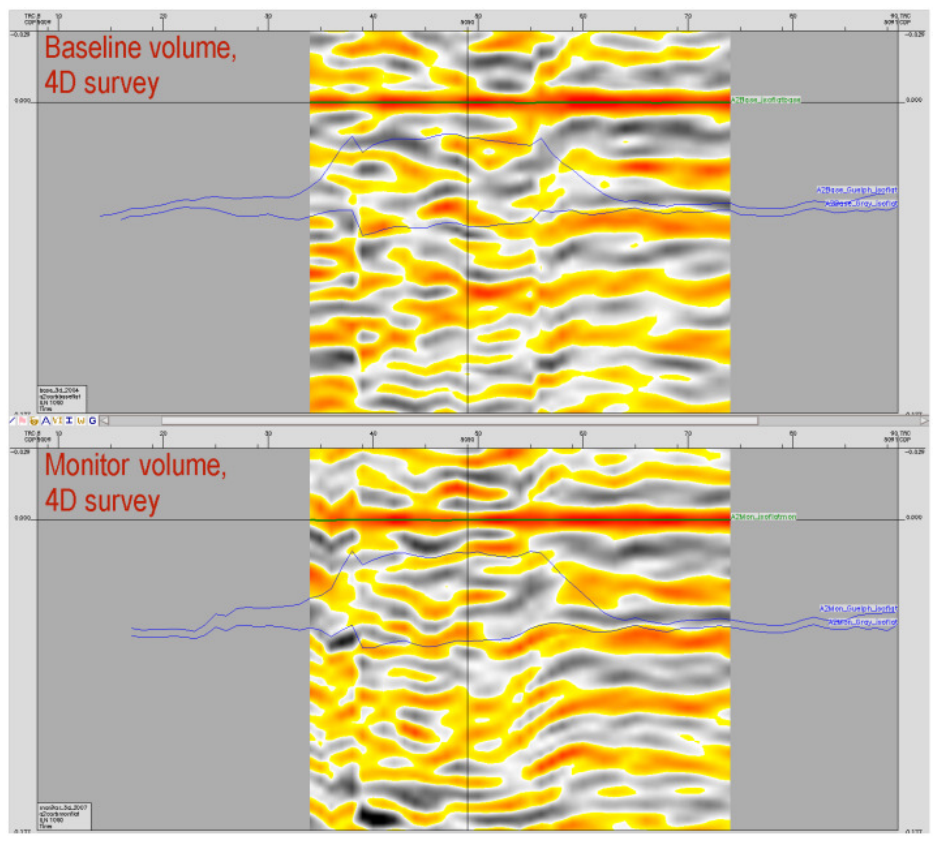

Figure 21: Comparison of In-line 1060 from the 4D seismic survey. chapter.

In addition to the time-lapse p-wave data set an attempt was made to acquire a small, 3-D multicomponent data set at the same time the Monitor P-wave survey was being acquired. A 48 channel Geometrics Strataview system with 24 3-component 40/100Hz Geophones was obtained through the Geoscience Department of Michigan State University and deployed within the center of the Charlton 30/31 field. It was hoped that an electrical signal cable could be run between the doghouse of the P-wave survey and the Geometrics Strataview system in order to synchronize the two systems. This would have allowed the seismic energy from the dynamite shots prepared by the P-wave survey crew to be used as energy sources for the multicomponent acquisition. 
Both the P-wave survey doghouse and the multicomponent system were stationed at the same time at the surface location of the $\mathrm{CO}_{2}$ injector well. A number of attempts were made to synchronize these two acquisition systems in order to ensure that their recording clocks started with the trigger of the dynamite shot. Unfortunately, all attempts to do this in an automatic manner failed due to equipment incompatibility. In order to resolve this issue the multicomponent acquisition crew triggered the recording of each shot manually once the triggering tone was heard by radio.

This method proved to be highly inefficient and inaccurate. As a result of these inaccuracies as well as the project timeline the multicomponent data that was recorded was not analyze during this study.

\subsubsection{Newly Drilled Well Data}

\section{State Charlton \#4 - 30}

During the summer of 2006 plans were made for the drilling of the first new well in the Charlton 30/31 field since the ending of its primary production development phase. This well, the State Charlton \#4 - 30, was being drilled in conjunction with a $\mathrm{CO}_{2}$ sequestration study project that was to take place in a shallower reservoir, the Bois Blanc Formation. This well was to be deepened to test the reef's western edge. Figure 19 shows the location of this well.

The drilling of this new well provided an opportunity to test the porosity distribution model developed using the instantaneous frequency method. This method is described in detail later in this report in Chapter 3. Using this method I predicted that this well would have little to no porosity greater than 5\%. Additionally, the reservoir simulation, which was based on the porosity model that was developed with the initial "Baseline" 3-D survey, predicted that no $\mathrm{CO}_{2}$ would be encountered in the reef at this location and time.

The Charlton 4-30 well was drilled at the end of 2006. Well logging and sidewall cores were taken throughout the reef section. Results from the analysis of this data are discussed in Chapter 3. 


\section{Larsen 3-31 well}

The Larsen 3-31 well was drilled in May of 2008 on the eastern edge of the reef in the southern portion of the field. As with the State Charlton \#4-30 well location I examined the instantaneous frequency volume, extracted from the Baseline 3D survey, in the area where the Larsen \#3-31 well was to be drilled and I made a prediction concerning the amount of porosity that would be encountered at this location. I predicted that a zone of porosity greater than $5 \%$ would be encountered half way through the reef at this location. This well was drilled as planned and logged, however, no cores were obtained in this borehole.

\subsection{Initial Structural and Stratigraphic Investigations}

\subsubsection{Forward Ray-Trace Modeling}

In preparation for the acquisition of the Baseline 3D seismic survey geologic information obtained during the initial development of the field in the 1970s was obtained and loaded into a geophysical workstation. This information was used to develop a preliminary understanding of the field's geology. Figure 22 shows the original structure map based on the results of drilling that took place during the field's initial development. 


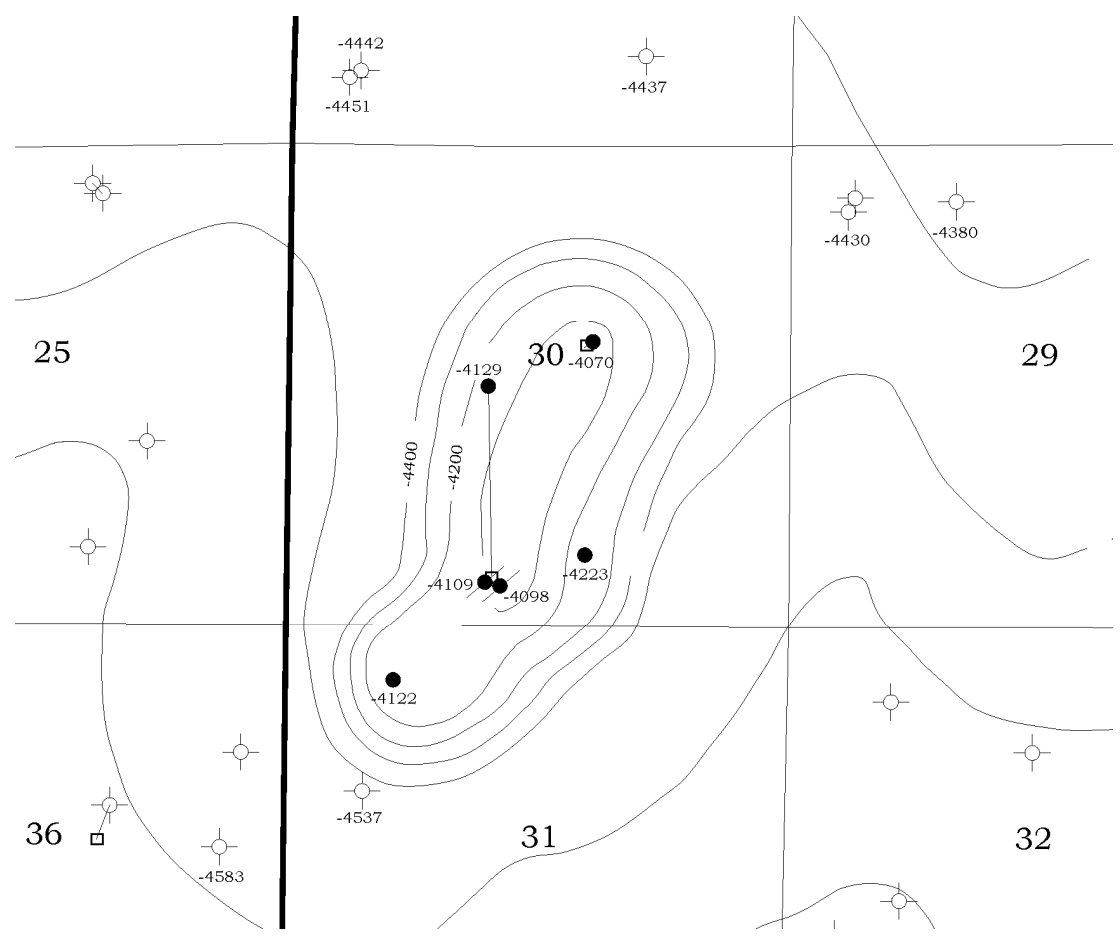

Figure 22: Original structure map of the Top of the Guelph formation based on the results of drilling that took place during the field's initial development and some 2D seismic lines.

This map was digitized and loaded into a GeoFrame, a geophysical interpretation program that is available for a UNIX-based workstation. As a result the structure of the Guelph reef was duplicated in the GeoFrame project, see Figure 23. The well logs from the six original wells drilled in the reef during the 1970s were transmitted to the Schlumberger office in Oklahoma City where they were analyzed and interpreted by a petrophysicist. The results of this analysis were also loaded into the GeoFrame project. These logs were interpreted and used to create a number of correlation sections and cross sections through the reef. One of these, constructed with the porosity logs, is shown in Figure 24. These logs were also analyzed to determine the petrophysical / geophysical properties of the various rock units from the shallow, near surface to the Niagaran Gray, the stratigraphic unit directly beneath the productive interval in the Guelph. Figure 25 shows the results of this analysis, which includes the shear-wave velocity which was calculated in AVOLOG a proprietary rock physics program available within Schlumberger. 


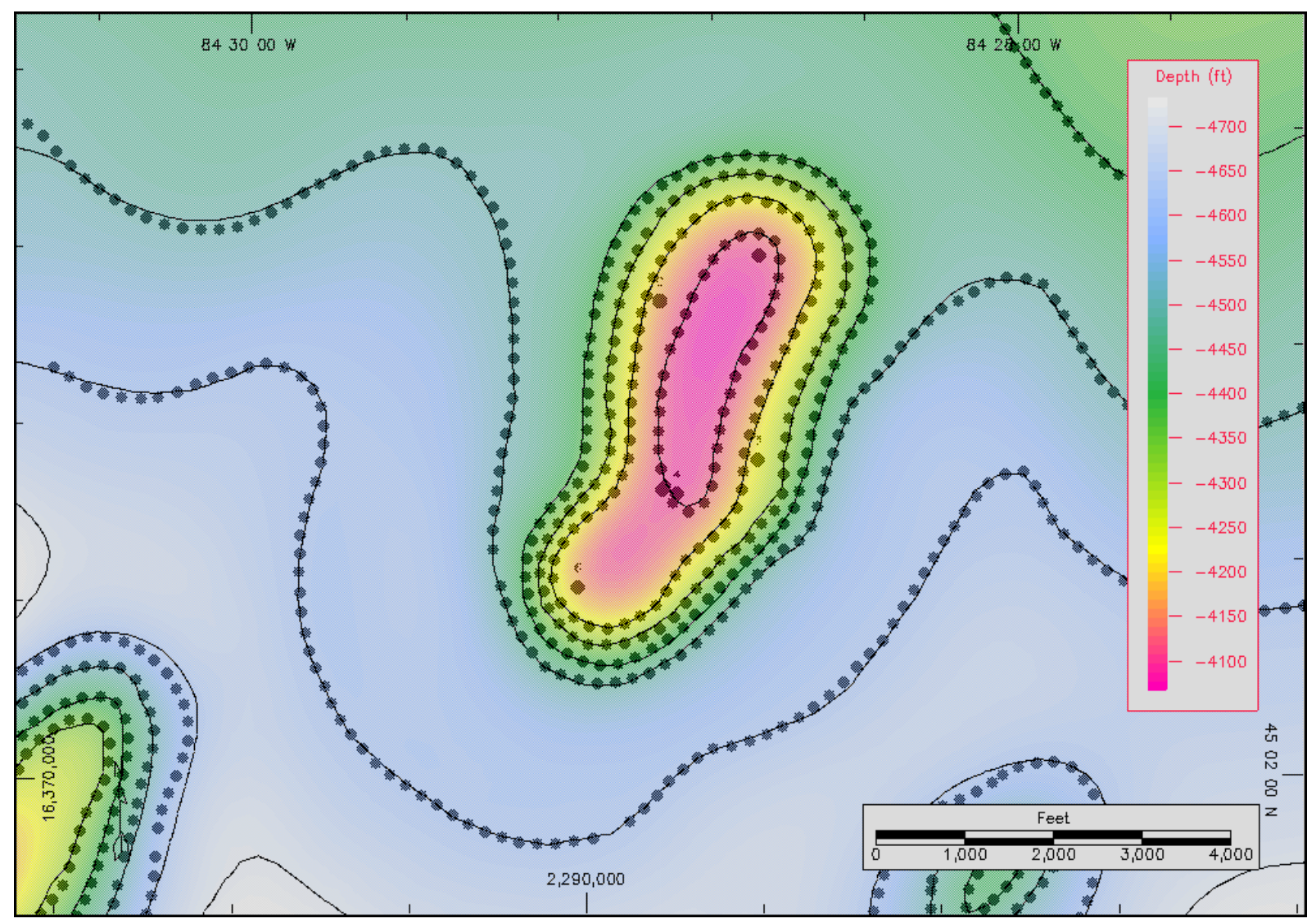

Figure 23: Original structure of the Guelph reef based on existing 2D seismic and well data at the start of the project. This was digitized into the GeoFrame project.

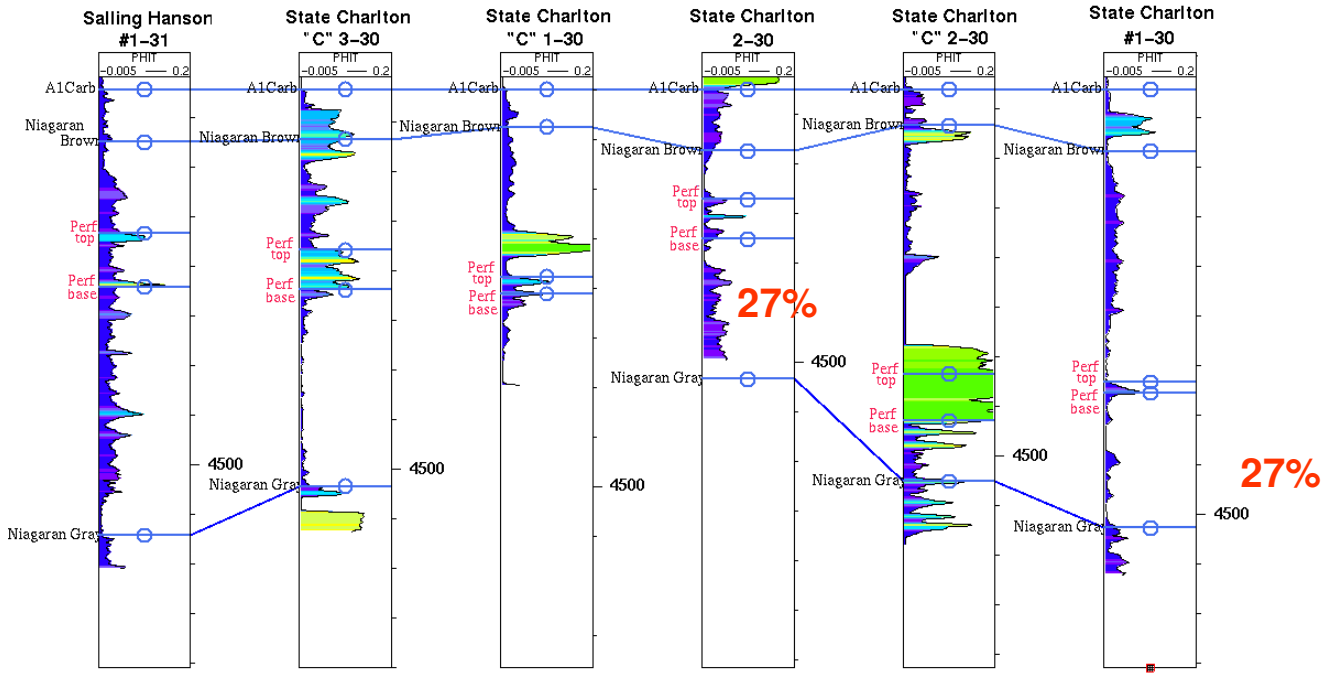

\section{Porosity scale $0-20 \%$}

Figure 24: Porosity log correlation section through the Charlton 30/31 reef. 


\begin{tabular}{|c|c|c|c|c|c|c|c|}
\hline \multicolumn{7}{|c|}{$\begin{array}{l}\text { Off Reef / On Reef Rock Property Combination } \\
\text { (On Reef Salling Hanson for deep units - Off Reef Black \#1-11 Gr and Dtc } \\
\text { and Weigandt Rhob for shallow units.) }\end{array}$} & \\
\hline & $\begin{array}{l}\text { Off / On Reef } \\
\text { Avg. GR. }\end{array}$ & $\begin{array}{c}\text { Off / On Reef } \\
\text { Avg. P-Vel. }\end{array}$ & \multicolumn{2}{|c|}{$\begin{array}{c}\text { Off / On Reef } \\
\text { Avg. S-Vel. }\end{array}$} & \multicolumn{2}{|c|}{$\begin{array}{l}\text { Off / On Reef } \\
\text { Avg. rhob }\end{array}$} & \\
\hline Antrim Shale & 121.6698827 & 12971.54761 & \multirow{9}{*}{ 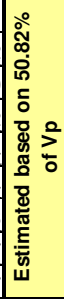 } & 6592 & \multirow{9}{*}{ 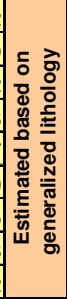 } & 2.5 & \\
\hline Traverse Fm & 54.22604839 & 16055.54182 & & 8159 & & 2.7 & \\
\hline Traverse Ls & 44.50418227 & 18697.56389 & & 9502 & & 2.65 & \\
\hline Bell Sh. & 110.5512097 & 10085.87022 & & 5126 & & 2.5 & \\
\hline Dundee & 25.20380309 & 17074.7634 & & 8677 & & 2.7 & \\
\hline Detroit River & 14.01371025 & 17294.4864 & & 8789 & & 2.3 & \\
\hline Base DR Evap & 22.83129652 & 19509.59483 & & 9915 & & 2.8 & \\
\hline Amherstburg Fm & 25.07425824 & 17941.4607 & & 9118 & & 2.8 & \\
\hline Bois Blanc & 23.9013271 & 17063.70587 & & 8672 & & 2.76 & \\
\hline Bass Is. & 25.8161321 & 19876.64786 & & 10112 & & 2.81 & \\
\hline Salina G & 47.54819475 & 17851.37263 & & 8586 & & 2.67 & \\
\hline Salina F & 27.6428653 & 18938.85858 & & 10946 & & 2.02 & \\
\hline Salina F Salt & 27.93511549 & 14547.30427 & & 7849 & & 2.21 & \\
\hline Salina E & 24.16752535 & 14124.51691 & & 7743 & & 2.22 & \\
\hline Salina D & 34.52321141 & 14605.79662 & & 7588 & & 2.44 & \\
\hline Salina C & 68.3775549 & 12257.22044 & & 5329 & & 2.47 & \\
\hline Salina B & & 1190.00000 & & 6047 & & 2.45 & \\
\hline Salina B Salt & 16.69636357 & 14896.94055 & & 8676 & & 2.14 & \\
\hline A2Carb & 24.62978261 & 20047.98441 & & 8817 & & 2.75 & \multirow{3}{*}{$\begin{array}{l}\text { On-Reef } \\
\text { Section }\end{array}$} \\
\hline A2Evap & 20.4262963 & 20118.85024 & & 9663 & & 2.87 & \\
\hline A1Carb & 25.29349057 & 20090.8257 & & 8735 & & 2.70 & \\
\hline A1Evap & 9.7566 / NA & 14921.16 / NA & \multicolumn{2}{|c|}{9110.04 /NA } & \multicolumn{2}{|c|}{$2.06 / \mathrm{NA}$} & \multirow{3}{*}{$\begin{array}{l}\text { On-Reef } \\
\text { Section }\end{array}$} \\
\hline Niagaran Brown & 14.385 & 20020.36071 & & 11525 & & 2.69 & \\
\hline Niagaran Gray & 26.45027523 & 19478.54679 & & 9448 & & 2.73 & \\
\hline
\end{tabular}

Figure 25: An On-Reef / Off-Reef comparison of rock properties in and near the Charlton 30/31 field.

It should be noted that the A1Evaporite is shown to be in the "Off-Reef" portion of Table 1 because it pinches out on the side of the reef and does not overly it. This is a significant observation as its absence from the crest of the reef places the A1Carbonate with its P-wave velocity of 20,090 feet per second directly on top of the Guelph (Niagaran Brown) with its 20,020 feet per second Pwave velocity. Additionally, the variation in rock density for these two units is very small. This indicates that virtually no acoustic impedance contrast should be expected at the top of the reef form compressional seismic energy. Figure \#26 displays a synthetic created during the well-to-seismic tie process which supports this. Very little acoustic impedance difference is noted at the top of the reef, the Niagaran Brown, compared to the top of the A2 Carbonate. 


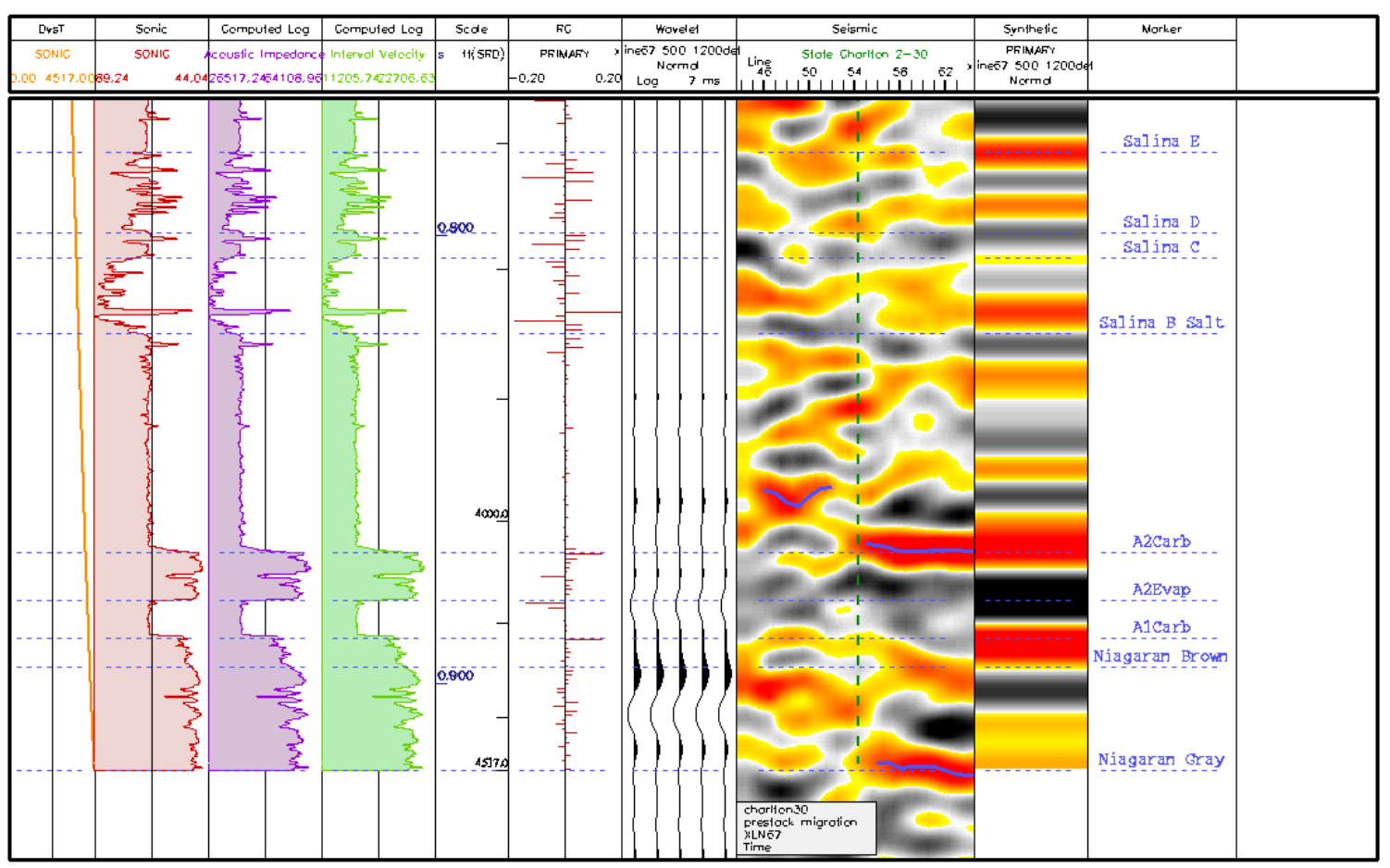

Figure 26: Well-to-seismic tie for the State Charlton \#2-30 well.

However, a strong acoustic impedance difference should be seen for S-wave (Shear-wave) energy at this same boundary. The implications are that the top of the Guelph reef should not be visible to the compression seismic energy (A1Carb p-wave velocity of 20,090 ft/sec over Niagaran Brown p-wave velocity of 20,020 ft/sec) but should be very visible in shear energy (A1Carb p-wave velocity of $8,735 \mathrm{ft} / \mathrm{sec}$ over Niagaran Brown p-wave velocity of 11,525 ft/sec), see Figure 25 .

Using the values shown in Figure 27a 12 layer rock property model was constructed in Gemini, a forward seismic modeling program. An exploded view of this model is shown in Figure 27.

The 12 layer acoustic model developed during the forward modeling process was used to test a number of 3D seismic acquisition geometries. During this process various 3D shot / receiver pairs were ray traced in order to predict various seismic responses from the reef. Figure 28 through Figure 30 show some of the model runs that were tested and the results. As this model (shown in Figure 27) 
was made prior to the acquisition of any actual surface seismic data an idealized, zero-phase wavelet was used for the forward modeling.

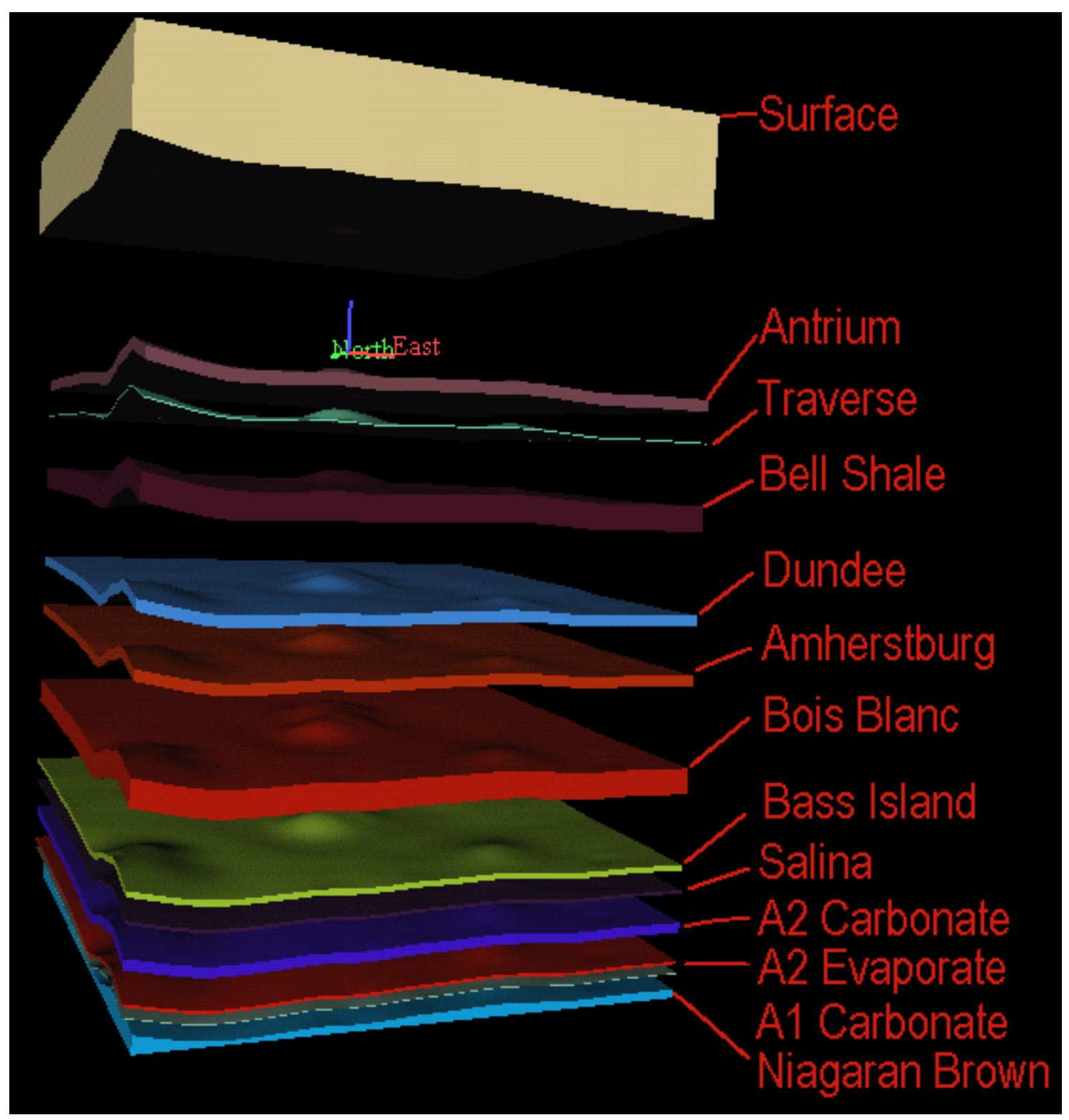

Figure 27: Exploded view of the 12 layer forward model in Gemini constructed using the rock properties shown in Figure 25. 


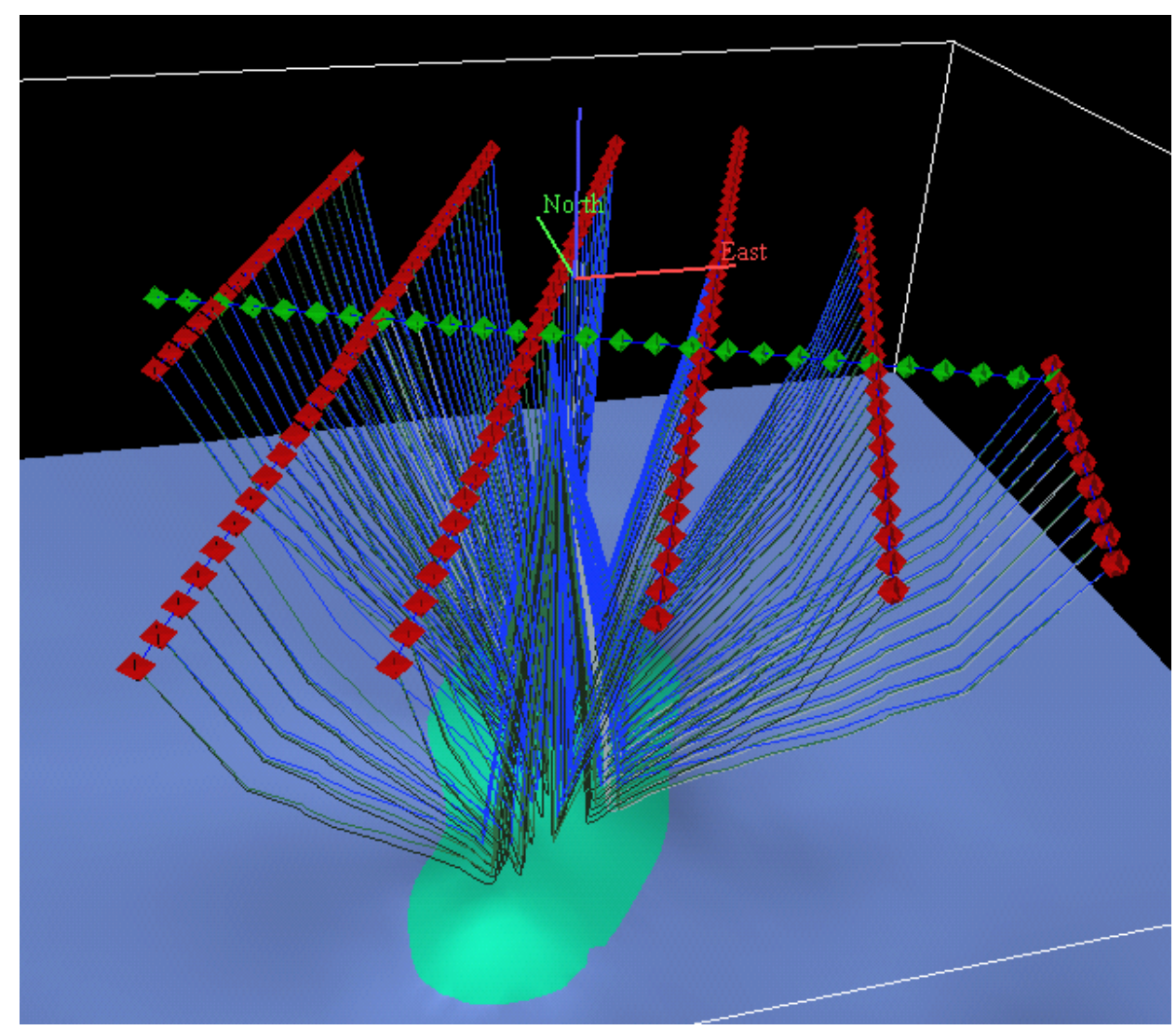

Figure 28: Display from one forward 3D seismic ray-trace model for multiple sources for a single receiver.

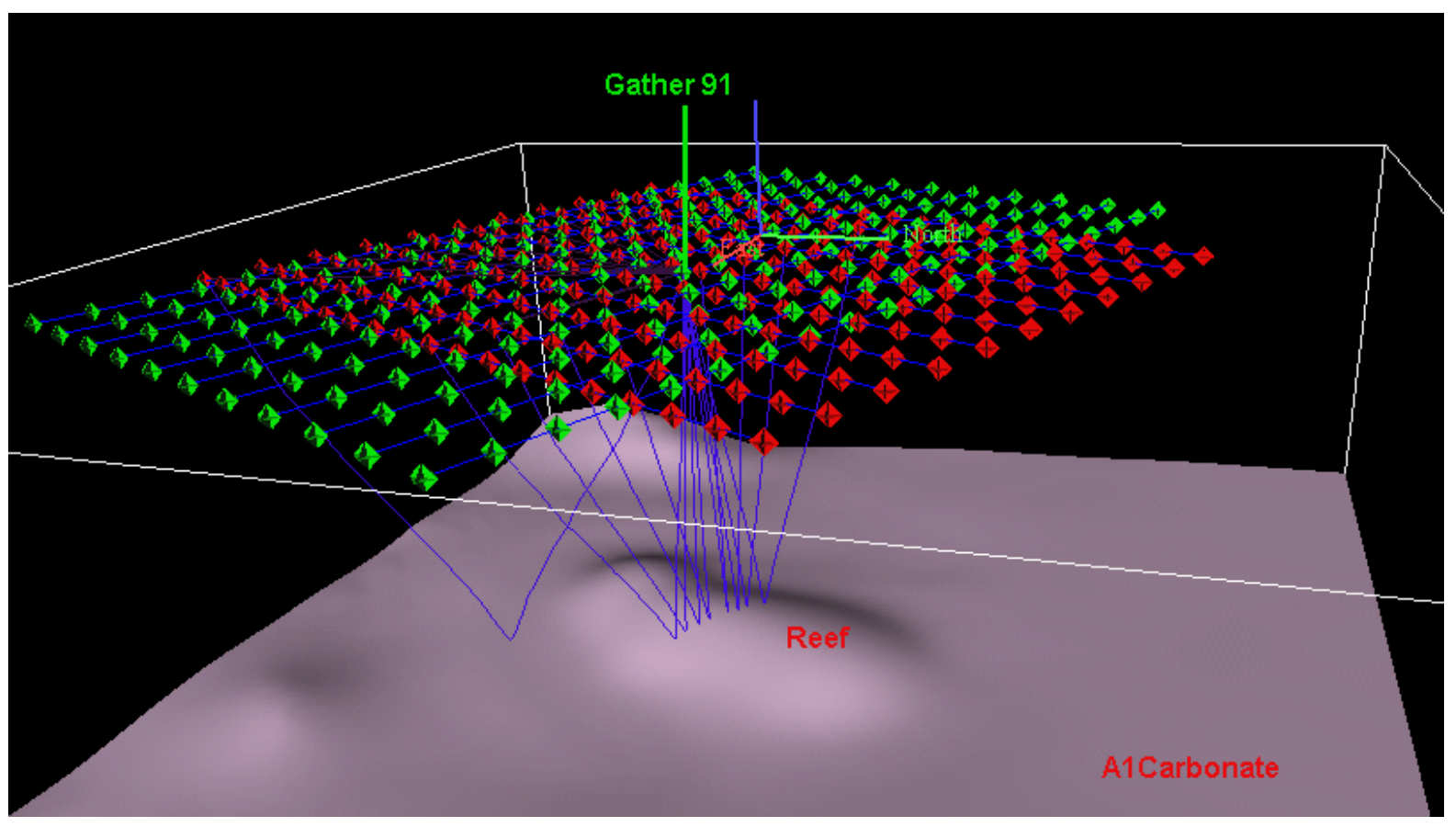

Figure 29: Forward 3D seismic ray-trace model for Gather 91. 

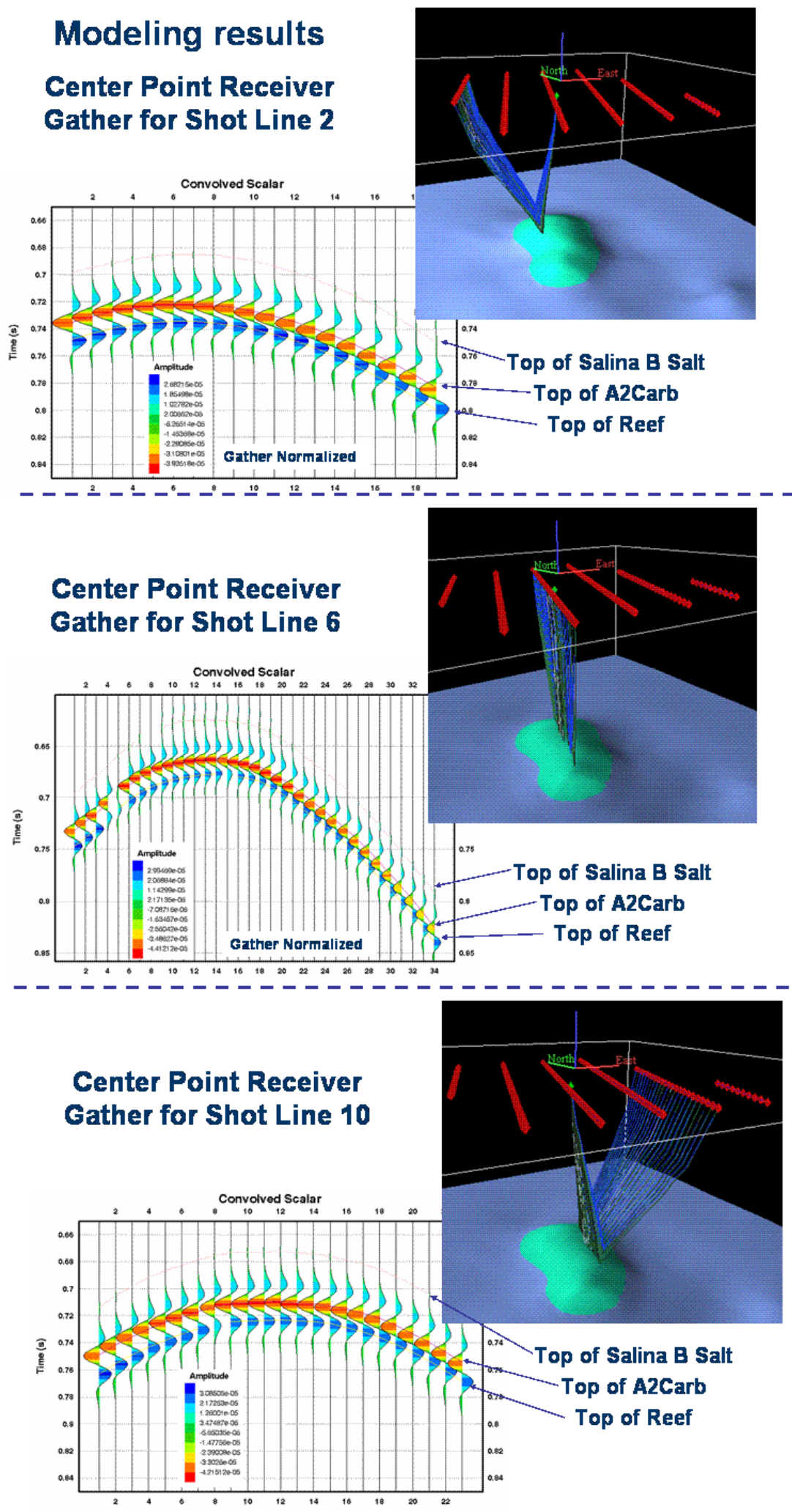

Figure 30: 3D ray trace modeling results showing predicted seismic response at top of reef. While multiple modeling runs were performed only the response of the lower units are shown in these figures. 
As a result of the modeling, the seismic acquisition parameters, which had been originally developed prior to the onset of the project, were altered to ensure the optimum imaging of the reservoir. Primarily a change was made in the orientation of the shot and receiver lines in order to obtain angles of incidence more perpendicular to the structure (in map view). The topography of the study area and the final acquisition geometry is shown in Figure 31.

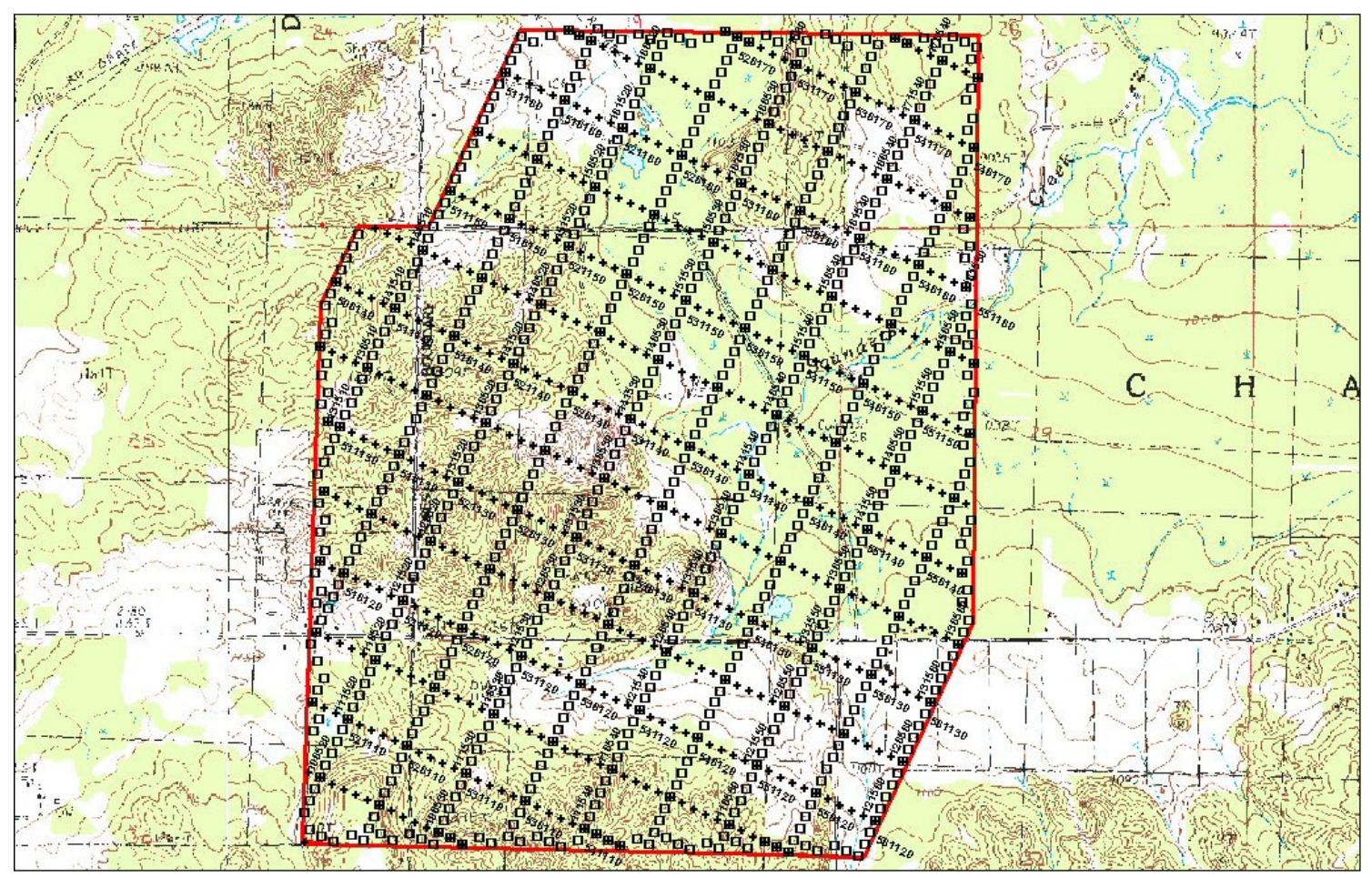

Figure 31: Topography and Final acquisition geometry of the Baseline 3D survey for the Charlton 30/31 field area. Open circles represent receiver locations and the plus signs are energy source point locations.

\subsubsection{Well-to-Seismic Tie Generation}

Once the baseline survey had been acquired and processed a number of geophysical analyses were performed on the resulting seismic data set. Wavelet analysis was conducted in a number of locations within the volume in the vicinity of those wells that had acquired sonic logs. A deterministic method was used for this wavelet extraction which includes the reflection coefficients (RC) calculated from the well log data. The extracted wavelets were combined with the log data to produce good quality well to seismic ties. For this analysis both a long and short seismic time 
window were used for the extraction of the wavelet. Figure 32 shows a screen capture of the Plate 1 montage created for the Department of Energy report, Toelle (2009) that documents the results of the short windowed wavelet extraction. Components of the plate are shown in later figures.

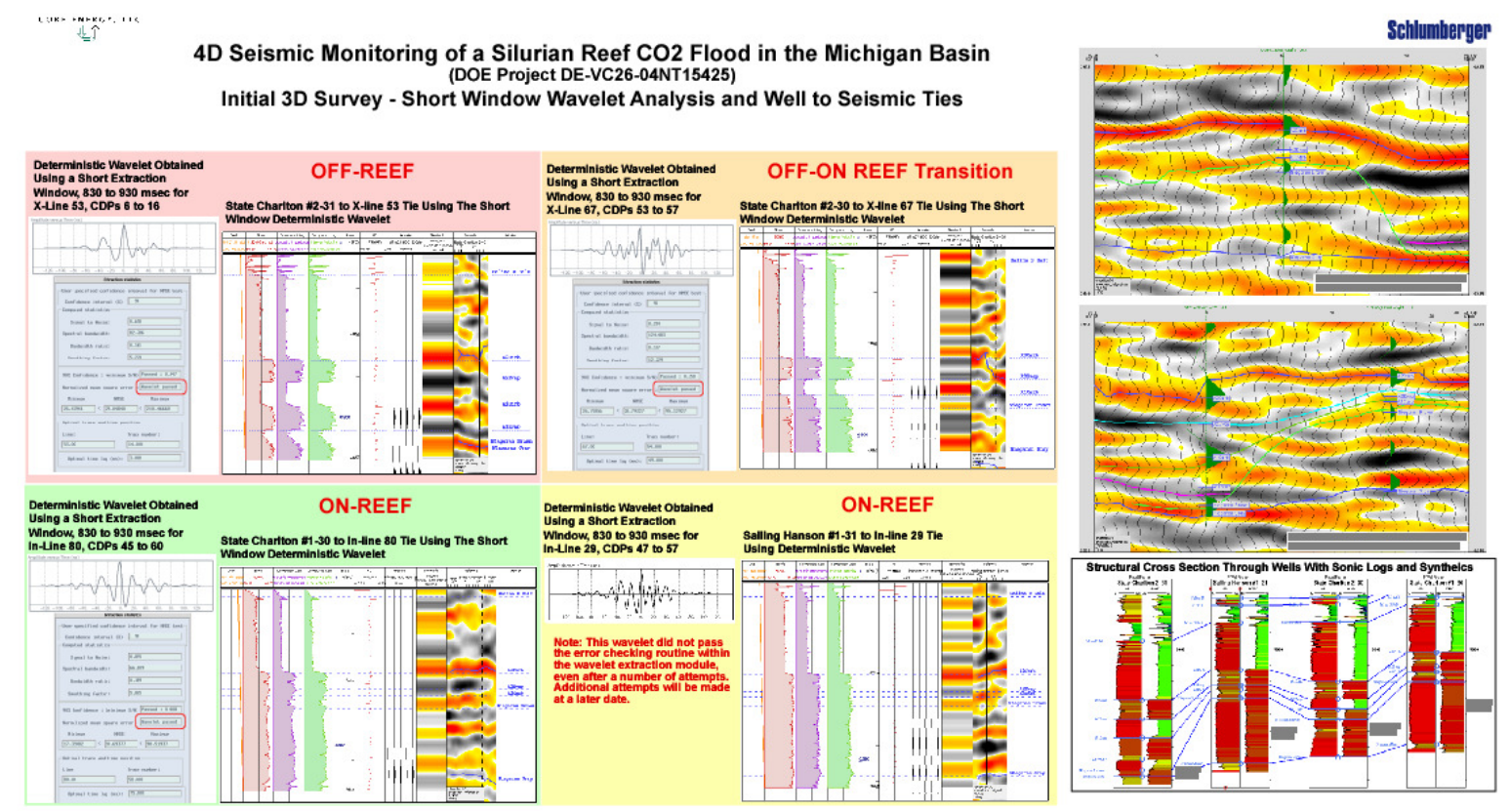

Figure 32: Screen capture of Plate 1 illustrating the results of the short windowed wavelet extraction.

In this display two wavelets were extracted from the baseline seismic volume and used in the development of synthetic seismograms which, in turn, were used for the well-to-seismic ties. Figure 33 shows the short windowed wavelet extracted from cross line 67 over a window from 830 to 930 msec in the vicinity of the State Charlton 2-30 well. This wavelet displays many characteristics of a minimum phase wavelet, as expected for the type of survey acquired. The synthetic seismogram that was generated with this wavelet is also shown in this same figure.

Figures 33 and 34 show the well to seismic tie developed with this synthetic. In this display the seismic is displayed in the red-yellow-white-gray-black color map where red is a strong positive reflection event (or peak) value and the black is a strong negative (or trough) value. The synthetic is shown in green as a traditional wiggle trace. This tie was judged to be "fair" in quality. 


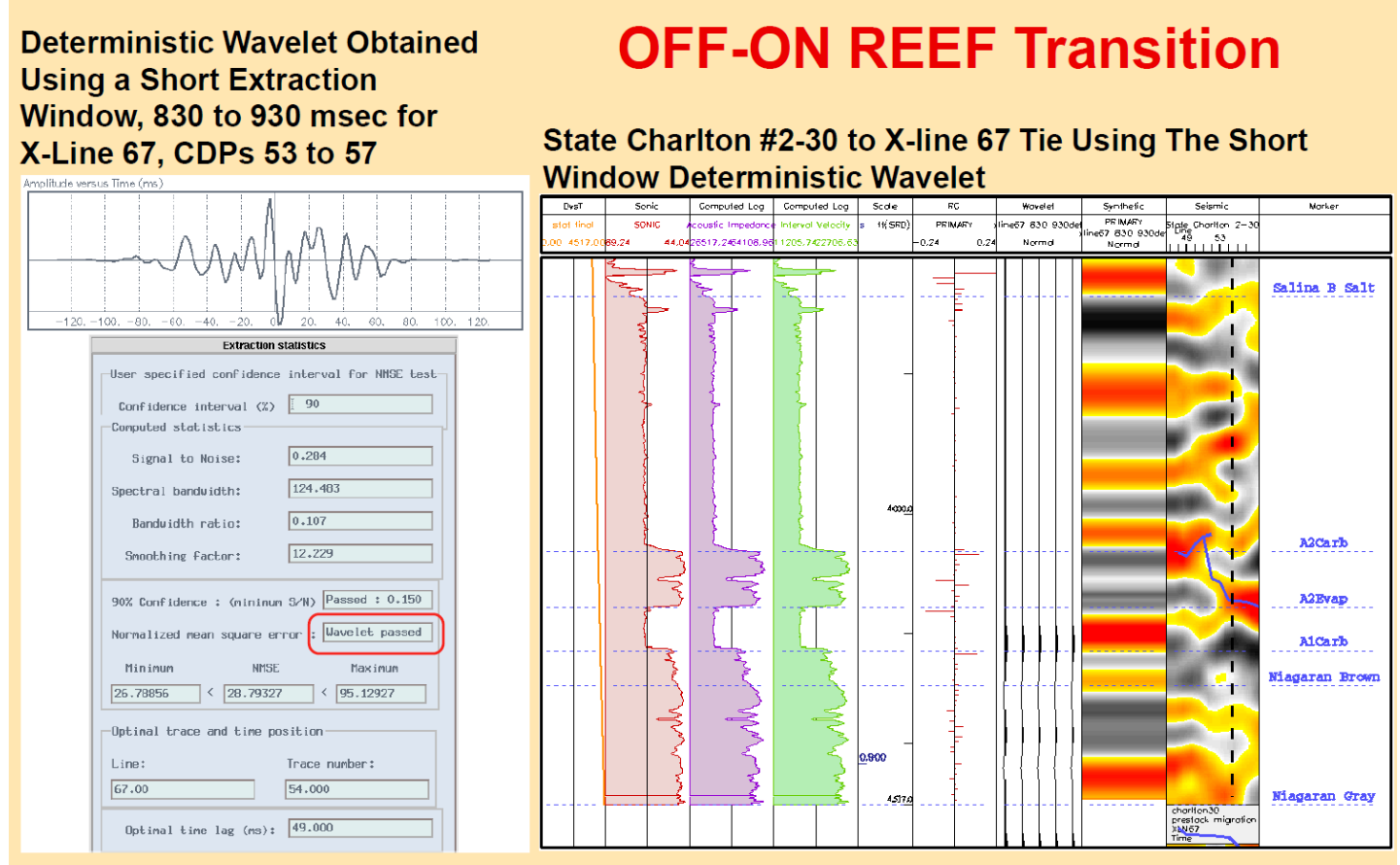

Figure 33: The short windowed wavelet extracted from the vicinity of the State Charlton 2-30 well.

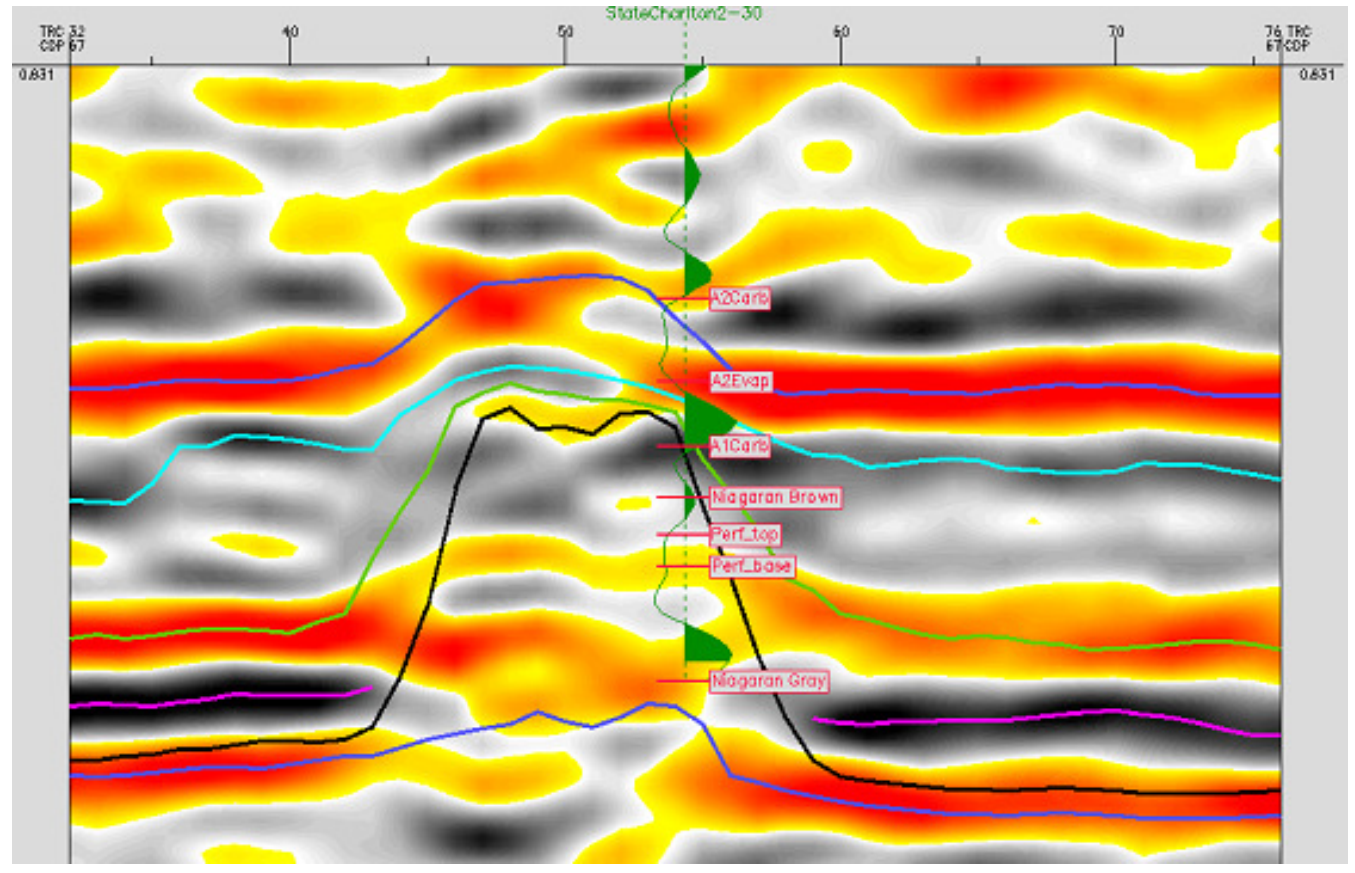

Figure 34: Well to seismic tie developed with the short windowed extracted wavelet for the State Charlton \#2-30 well 
Figure 35 and Figure 36 show the same information as in the previous figures, but for the State Charlton 1-30 well and its well-to-seismic tie on in-line 80. This tie was judged by me to be of "excellent" quality due to the its character tie with the seismic trace data.

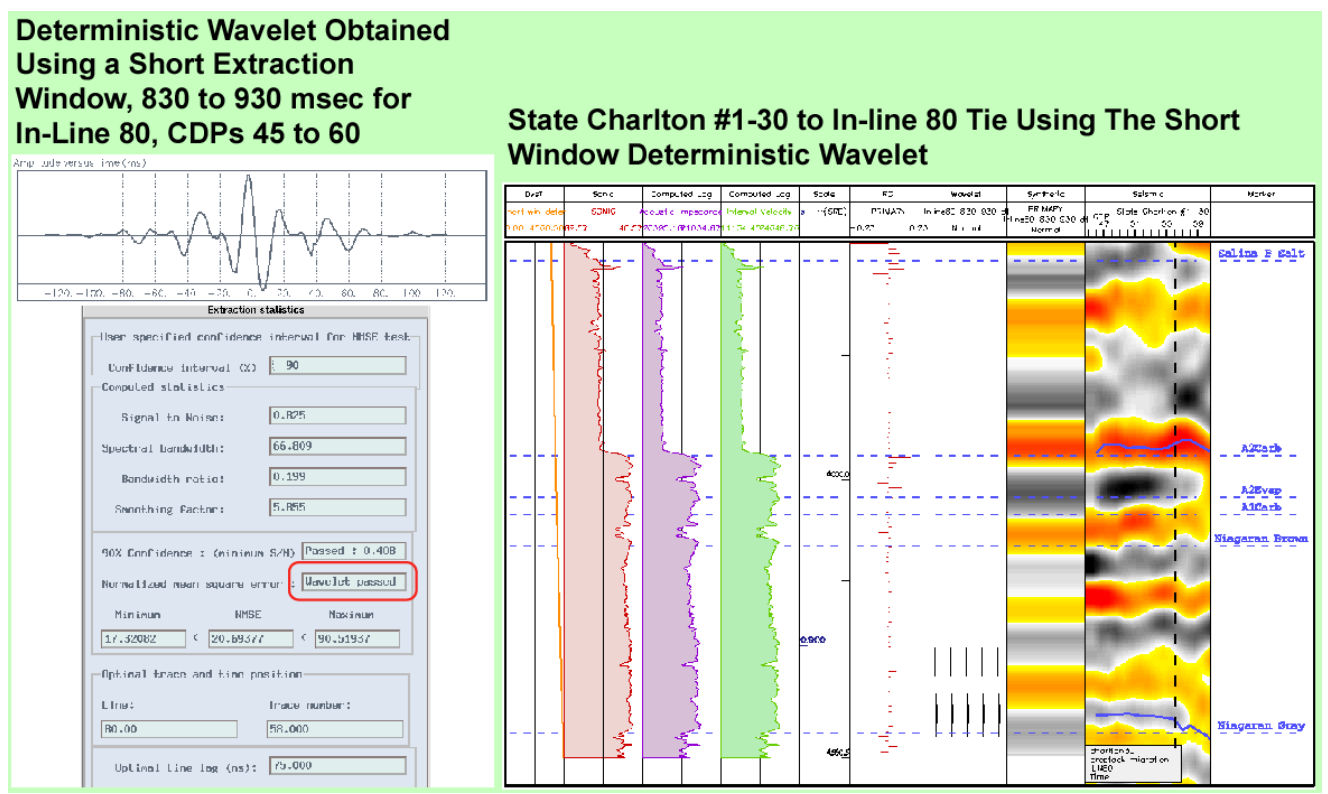

Figure 35: The short windowed wavelet extracted from the vicinity of the State Charlton 2-30 well and the synthetic seismogram developed using this wavelet.

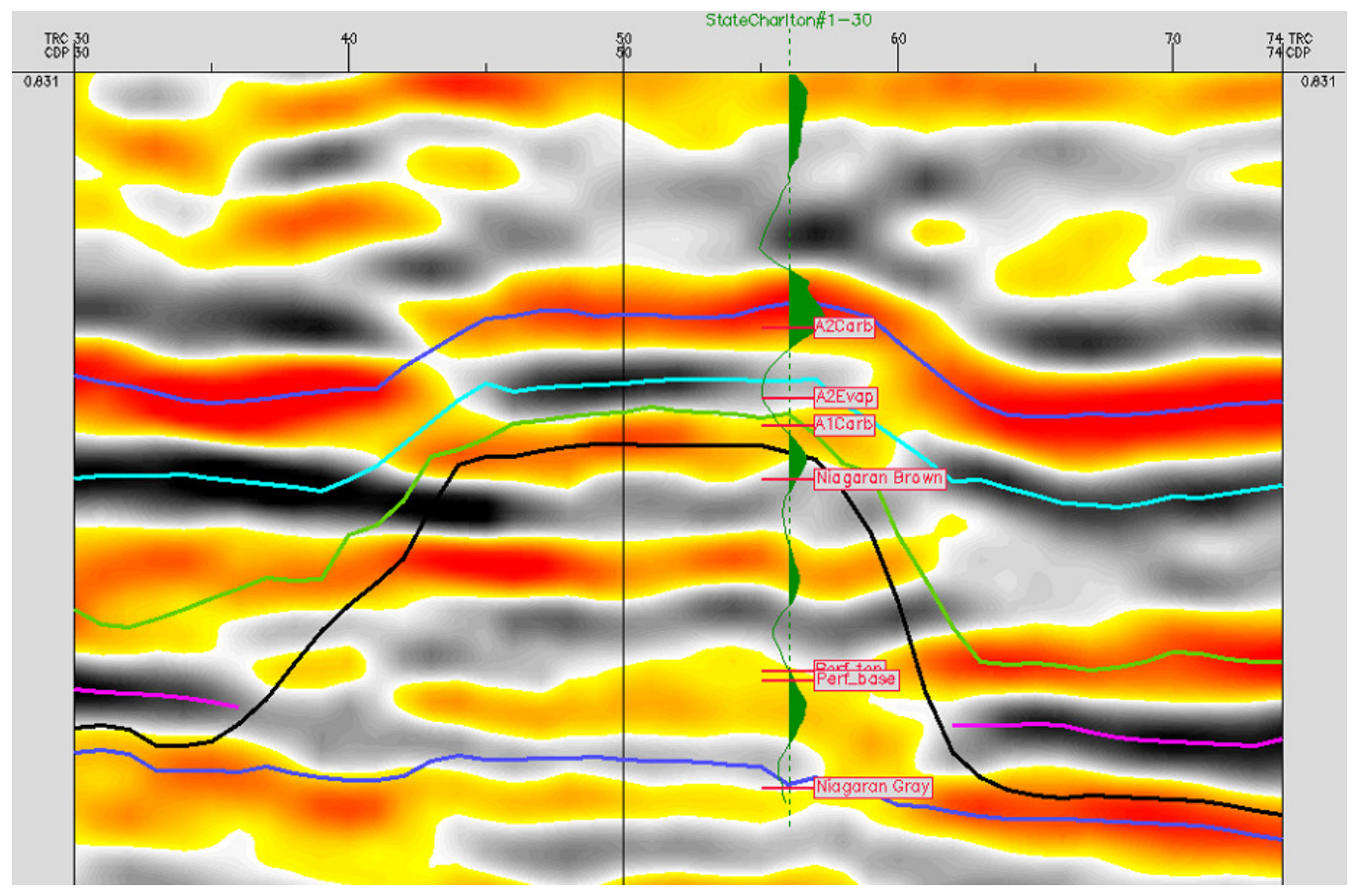

Figure 36: Well to seismic tie developed with the short windowed extracted wavelet for the State Charlton \#1-30 well. 


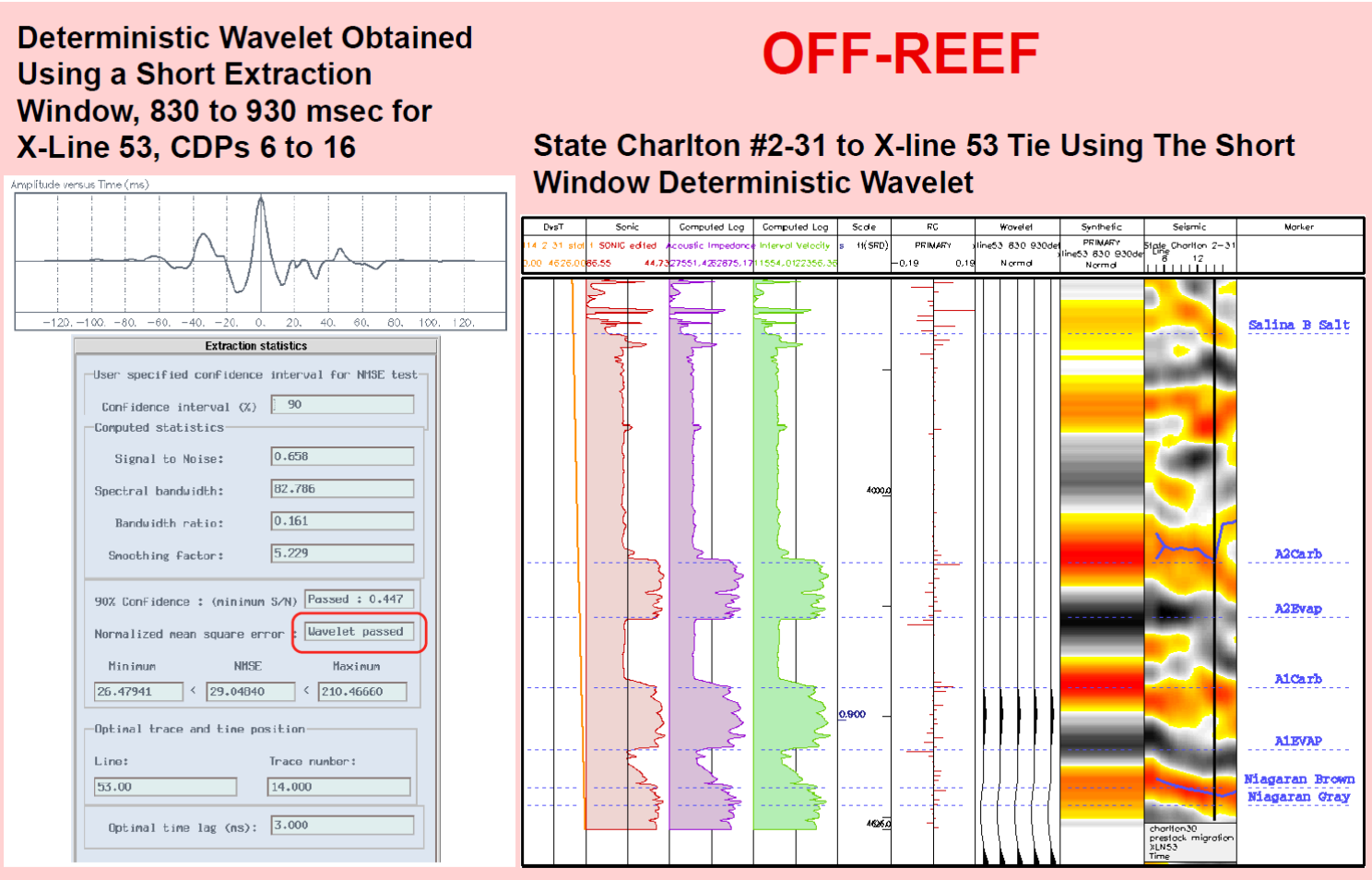

Figure 37: The short windowed wavelet extracted from the vicinity of the State Charlton 2-31 well (off-reef well) and the synthetic seismogram developed using this wavelet.

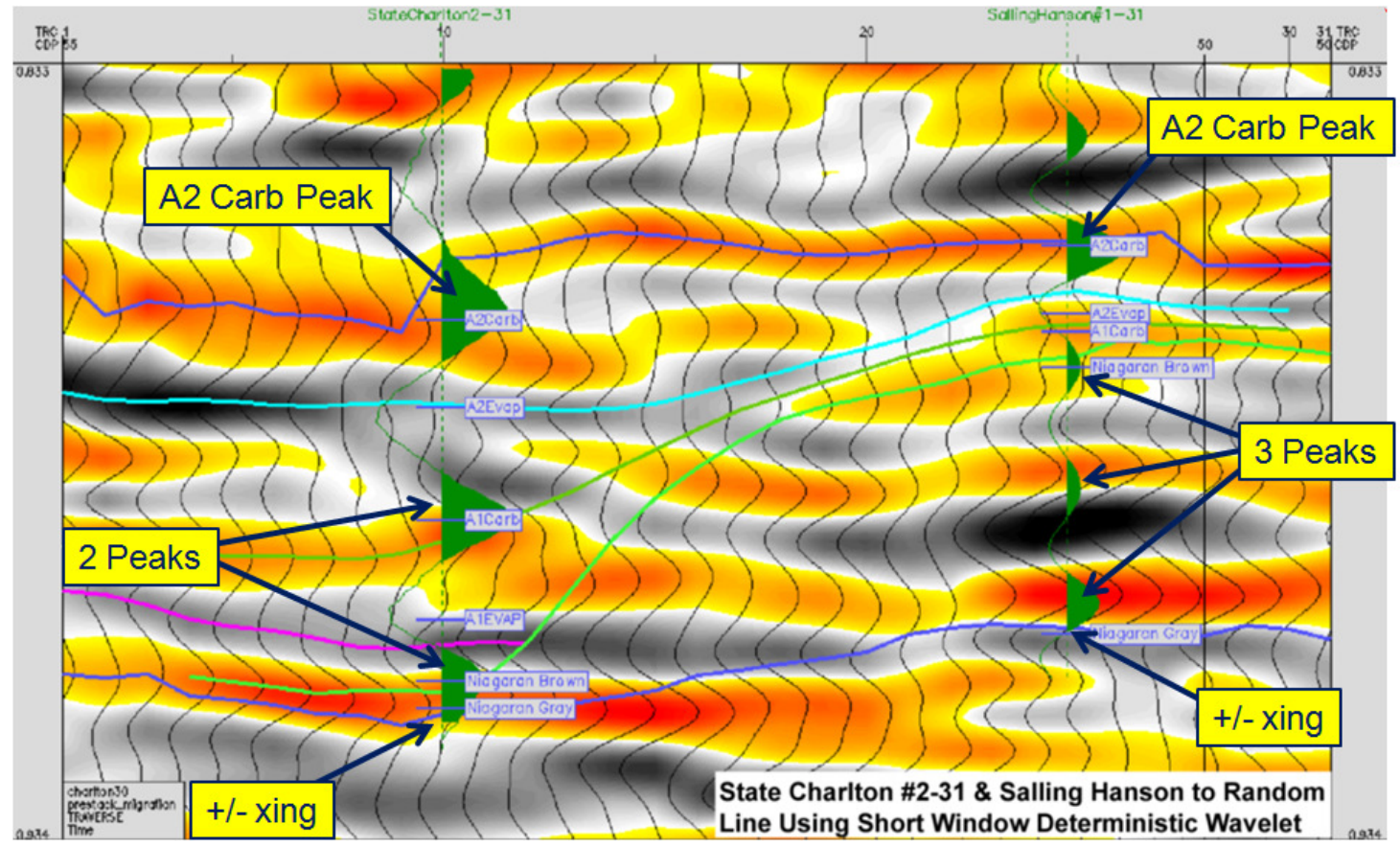

Figure 38: Well-to-seismic tie along a random line developed with the short windowed extracted wavelet for the State Charlton \#2-31 well and the Salling Hanson \#1-31 well. 


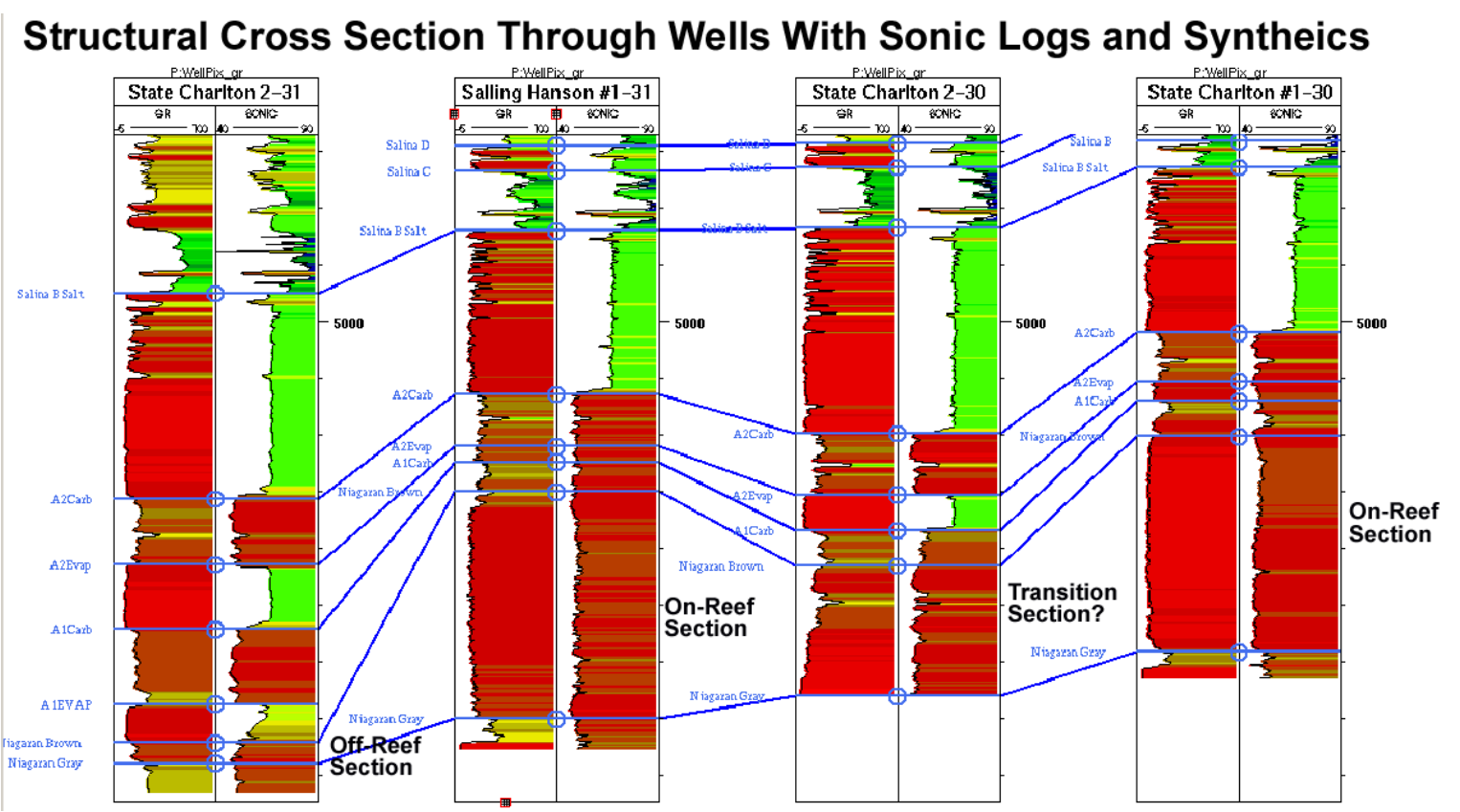

Figure 39: Correlation section showing all wells with sonic logs. These wells were use it the construction

Two well-to-seismic ties were generated, one for the State Charlton \#2-31 well (an off-reef well) and another for the Salling Hansen \#1-31 well (an on-reef well). Both of these wells are located in the southern part of the seismic survey (see Figure 19). Figure 38 shows a random line that runs through both wells, and the resulting well-to-seismic ties. Both of these ties are judged to be "good" to "very good" in quality. However, it should be noted that while the wavelet extracted for the State Charlton 2-31 well passed the software's quality control check, the one extracted for the Salling Hanson \#1-31 well did not pass, even though the resulting synthetic had a good character tie with the seismic. This display helps to illustrate the change in seismic character that was used to identify and map the reef.

In nearly all of the synthetics the top of the A2-Carbonate is the strongest peak event immediately above the top of the reef. This observation was to prove useful later in the study when the 4D analysis was conducted. In the Salling Hansen \#1-31 well three peak seismic events occur between the A2-Carbonate peak event and the reef's base, the Niagaran Gray, which is a positive to 
negative zero-crossing at this location (shown in Figure 38). In the State Charlton \#2-31 well, the only off-reef well with a sonic log and hence the only off-reef well for which a synthetic could be developed, only two peak events occur between the A2-Carbonate and the Niagaran Gray, also a positive to negative zero-crossing (also shown in Figure 38).

This change in seismic character, from three peaks on-reef to two peaks off-reef, proved to be consistent across the study area. The well-to-seismic tie for the State Charlton \#1-30 in the northern portion of the field also exhibits this same on-reef seismic character. This change was used to distinguish between the on and off-reef portions of the Niagaran Brown (Guelph) reef. This change was identified and mapped within the Baseline 3D survey, see Figures \#34, \#38 and \#42.

This same well-to-seismic tie analysis was performed using wavelets extracted over a much longer time window, 700 msec. Figure 40 shows a screen capture of Plate 2 from the Dept. of Energy study which documents the results of the long windowed wavelet extraction. The well-to-seismic ties that were generated from this analysis did not tie as well to the seismic data as those generated using the wavelets extracted over the shorter time window. Figure \#26 is taken from this illustration.

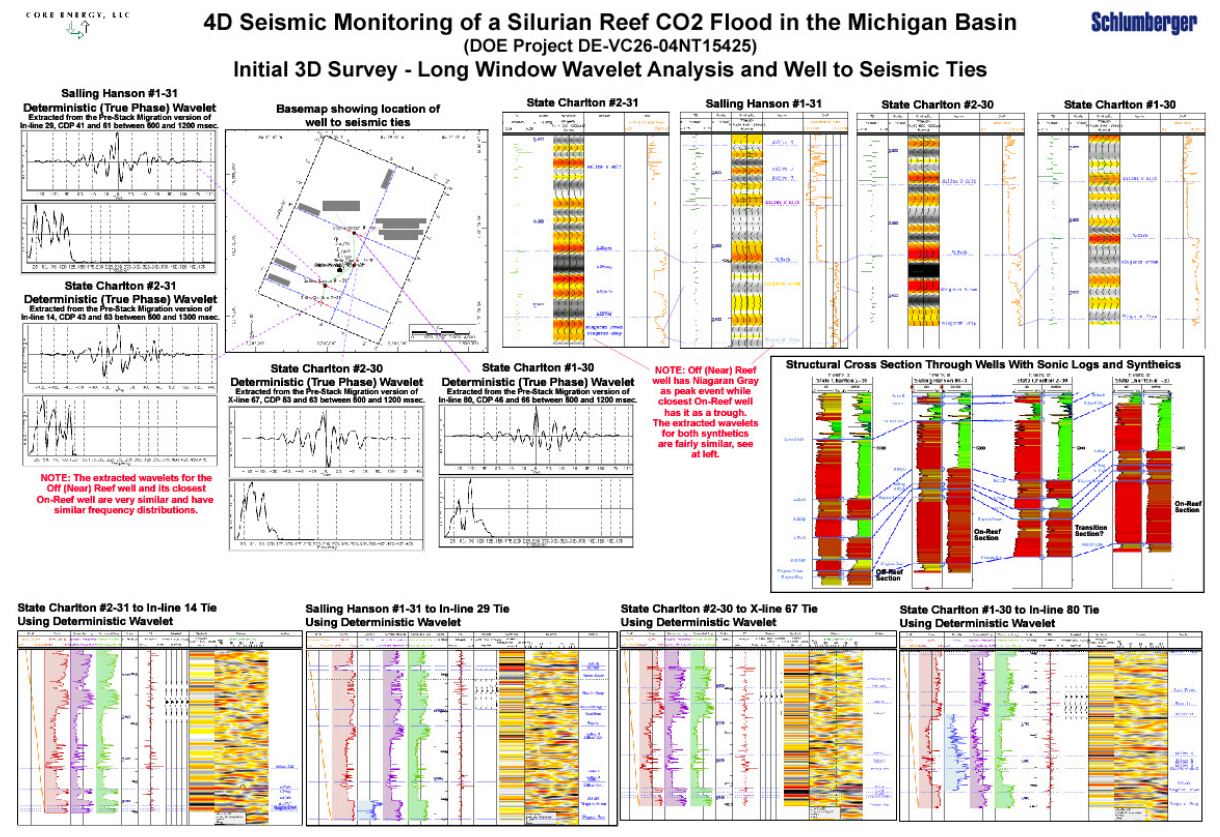

Figure 40: Screen capture of Plate 2 illustrating the results of the long windowed wavelet extraction. 
Figure \#41 shows that portion of Plate \#2 that illustrates the extracted wavelets using the long windowed extraction approach and the location where they were extracted in the study area.

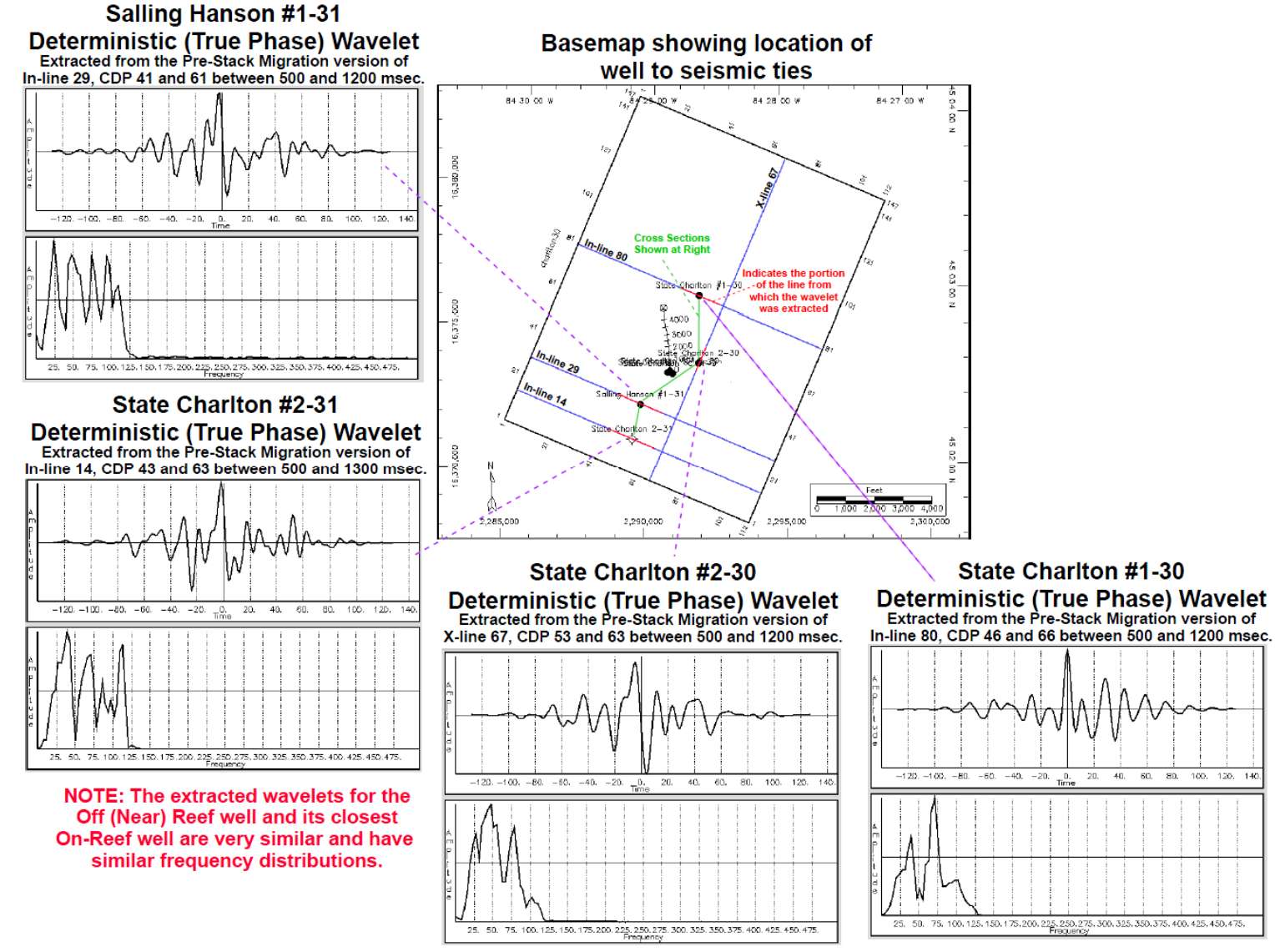

Figure 41: Wavelets extracted from the Baseline 3D survey using a long windowed extraction and their location within the study area.

In addition to the wavelet analysis a frequency analysis was also conducted. Figure 42 shows the frequency content of the seismic in the vicinity of each well-to-seismic tie as extracted during the long window wavelet analysis. The range of frequencies in the data shown in Figure 42 is fairly consistent at approximately 10 to $120 \mathrm{~Hz}$. 

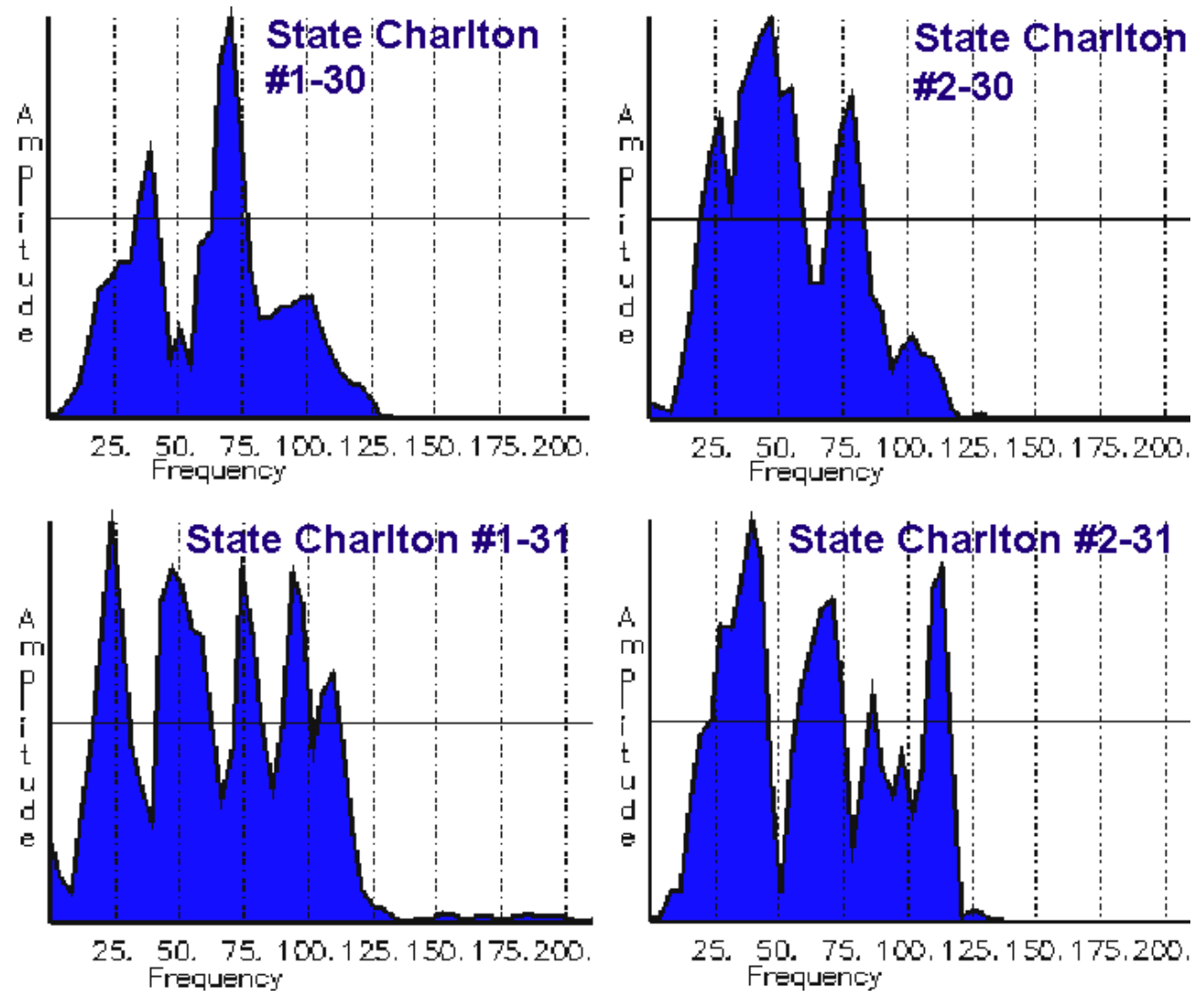

Figure 42: Frequency power spectrum for each well location extracted from the vicinity of the reservoir.

\subsubsection{Seismic Interpretation}

The synthetic seismic tie, while not excellent, identifies the major reflection events associated with reservoir intervals and provides the basis for seismic interpretation of the various horizons within the study area. The top and base of the Guelph reef (Niagaran Brown), as well as shallower horizons which help to establish the character tie at that location were interpreted on the seismic data. Figure 43 shows these horizons along one of the survey's crosslines, which is oriented approximately north northeast to south southwest. Travel time maps were developed for each of the interpreted horizons. Figure 44 shows the time map for the top of the Guelph reef. This figure displays both the time and depth contours in a single map. The time contours are in milliseconds and are shown with the purple contours. Depth contours are displayed in black. 
A velocity field was developed by combining information obtained during the well for seismic ties and comparing the horizon interpretation in time with the log data obtained in wells without sonic logs. This was used to convert the horizon time maps to depth, the black contours in Figure 44 for the top of the reef.

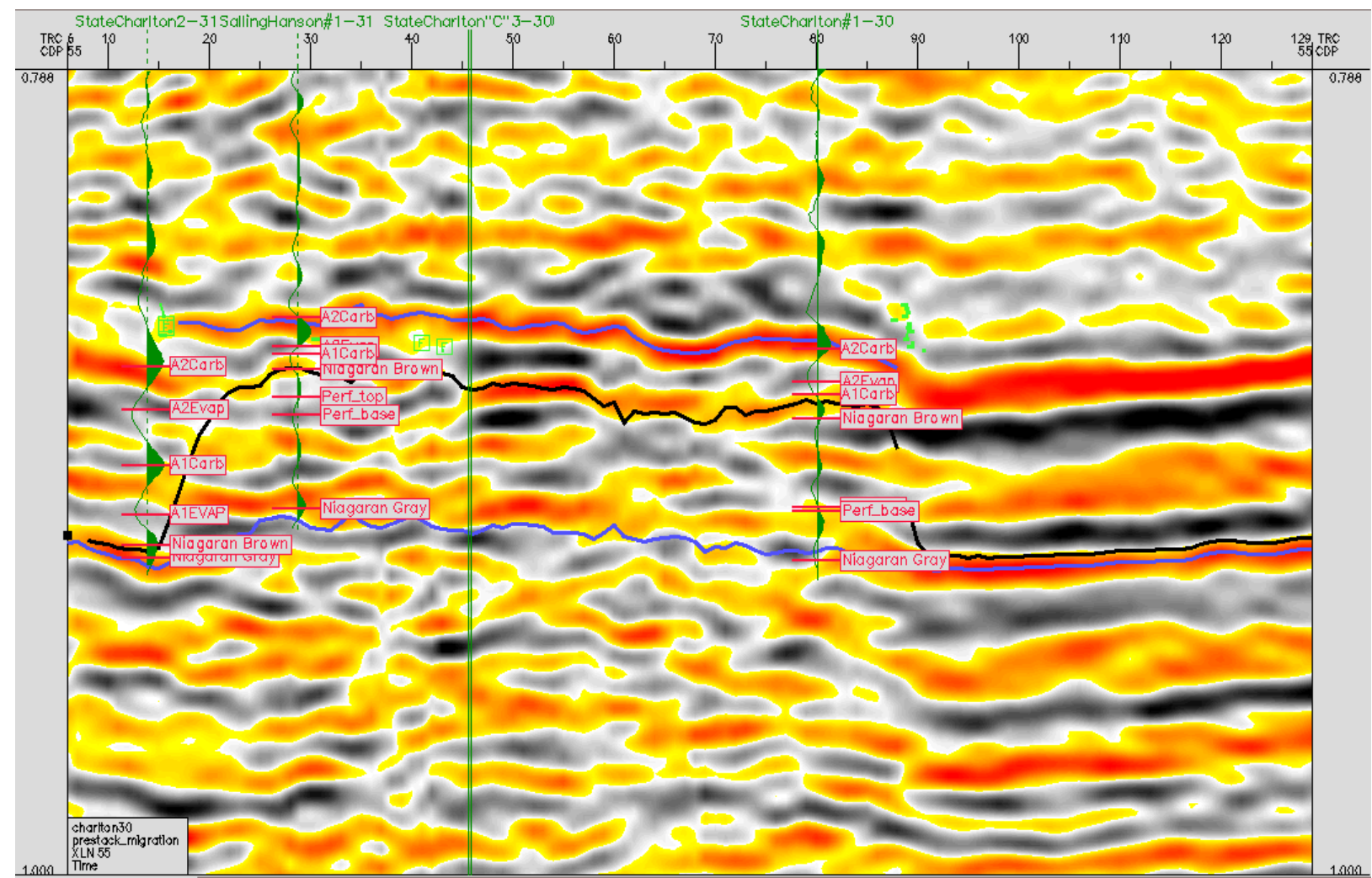

Figure 43: Crossline through the seismic volume at the reef level showing important seismic horizons interpreted during the study. 


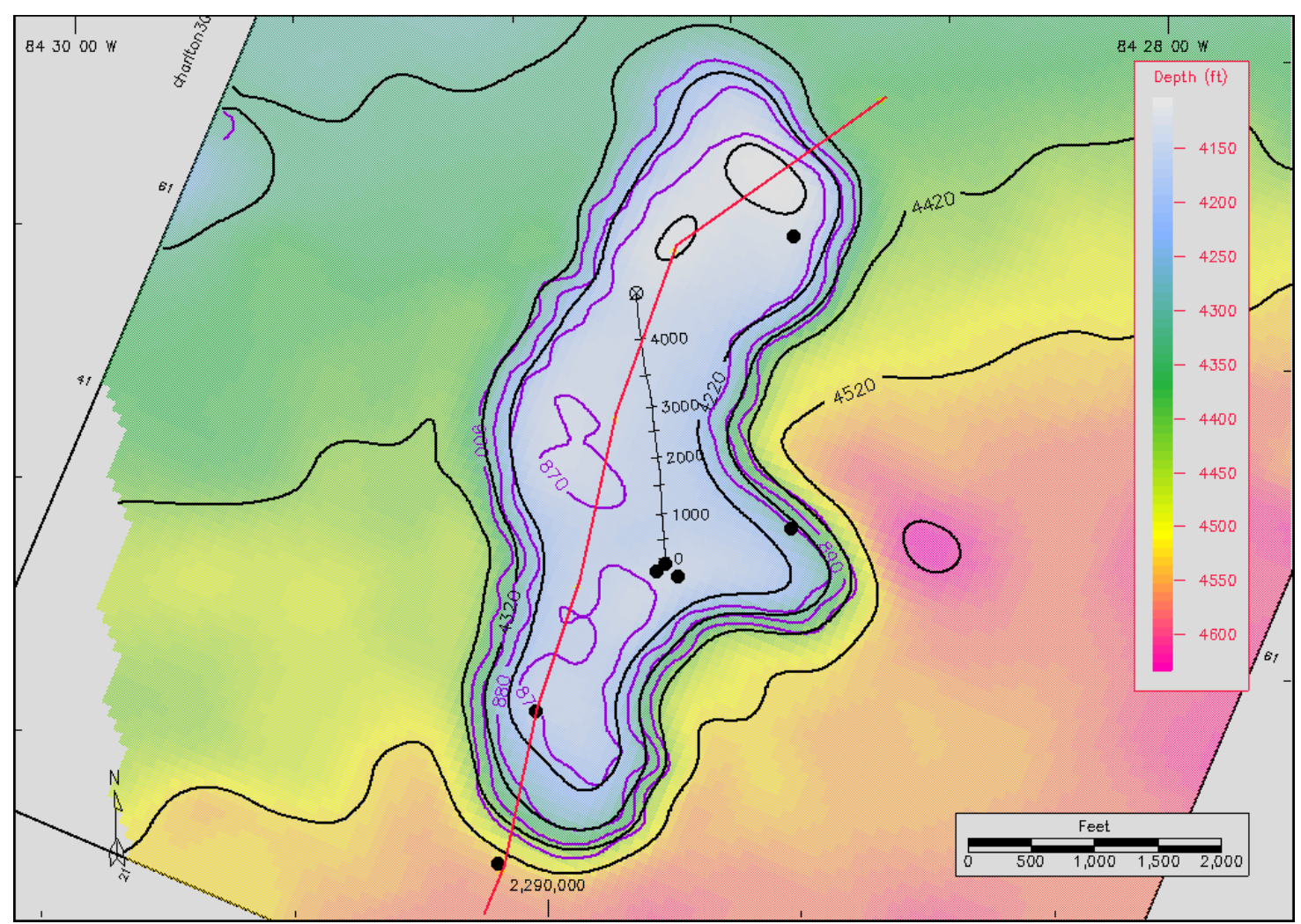

Figure 44: Time and depth map for the top of the Guelph formation. Time contours are in milliseconds and shown in purple.

The process of horizon interpretation (in the time domain), velocity analysis and then conversion to depth, described above for the top of the Guelph formation, was also performed for the other important horizons, such as the Niagaran Grey formation, needed to establish the reef thickness. This simple process of multiplying the horizon time with the velocity to convert the horizon to depth is readily applicable in this area due to the small size of the reef (only 300 acres) and the amount of well control within that small area (6 wells). With multiple horizons converted to depth the next step was the development of an isopach map derived from combined well and seismic data. This isopach map of the reef itself, from the top of the Guelph to the top of the Niagaran Grey formation, is shown in Figure 45 with the reef thickness values obtained from the logs. 


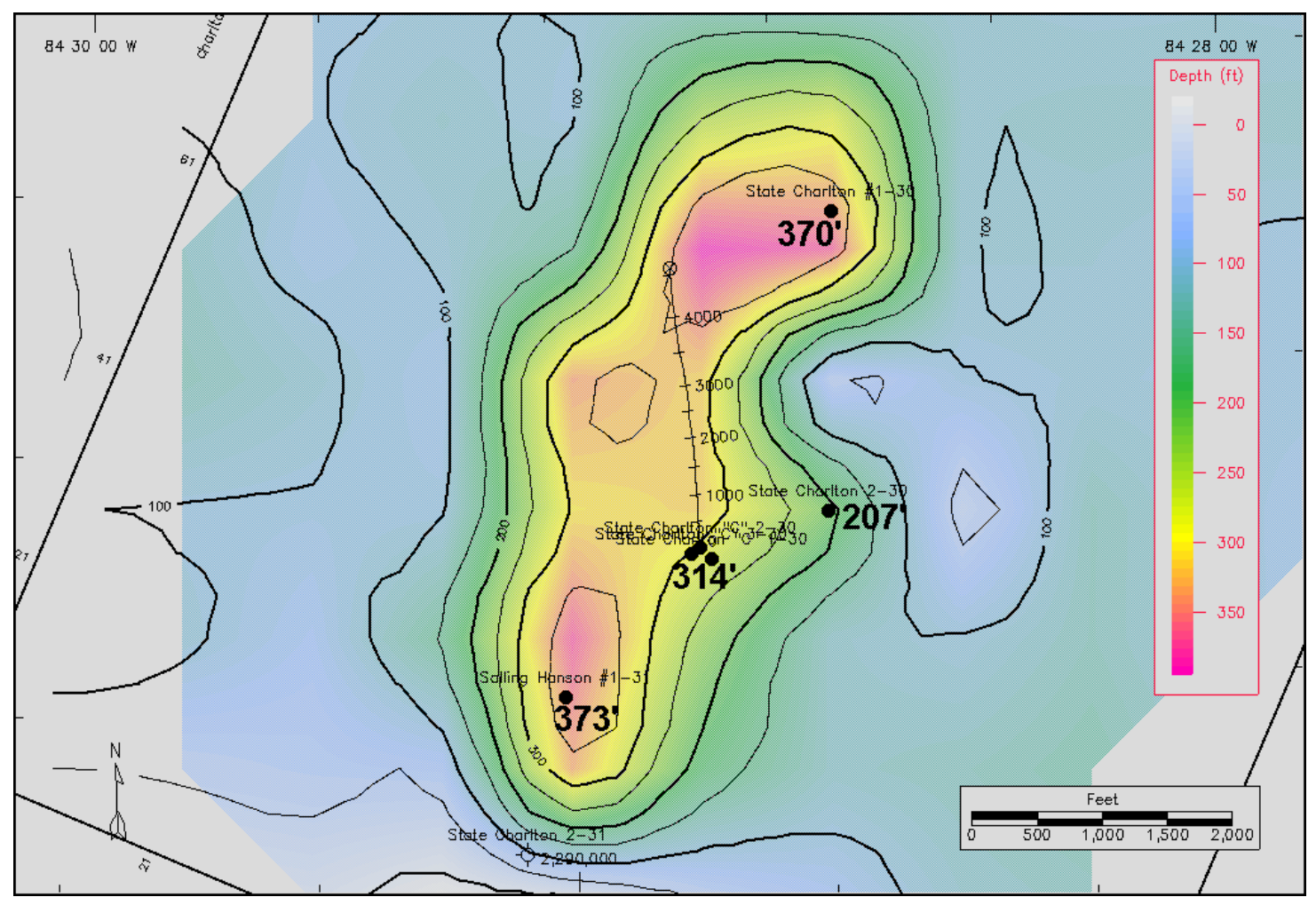

Figure 45: Isopach map of the Silurian Guelph reef at the Charlton 30/31 field.

\subsubsection{Seismic Attribute Analysis}

In addition to the well-to-seismic ties blended seismic attributes were developed in order to aid with this interpretation. Specifically, seismic variance and amplitude were found to be of value with this task. Seismic variance is a measurement of how rapidly the seismic data is changing. The higher the variance attribute that higher the rate of change. This attribute is often employed in order to identify locations in 3D seismic volumes where large spatial variations of reflection amplitude are located. Large signal variance is often associated with signal discontinuity that can have stratigraphic and structural origins, such as at a fault or a channel edge.

Seismic variance was extracted from the Baseline 3D survey using a $100 \mathrm{msec}$. time window and a 3 by 3 trace setting. Areas associated with high variance were visually blended (combined) with the high peak amplitude values to develop a composite display using the visualization program GeoViz. GeoViz was then used for the interpretation of the reef's edge. The edges on the reef were 
clearly visible with this method where high variance values bounded high amplitude values. Figure 46 shows a time slab from 855 to 860 milliseconds. The edge of the reef is clearly visible in the high variance shown in blue. High peak amplitudes are shown red-orange. Using these blended attribute displays greatly aided the identification and interpretation of the reef and led to a more detailed interpretation in a shorter amount of time.

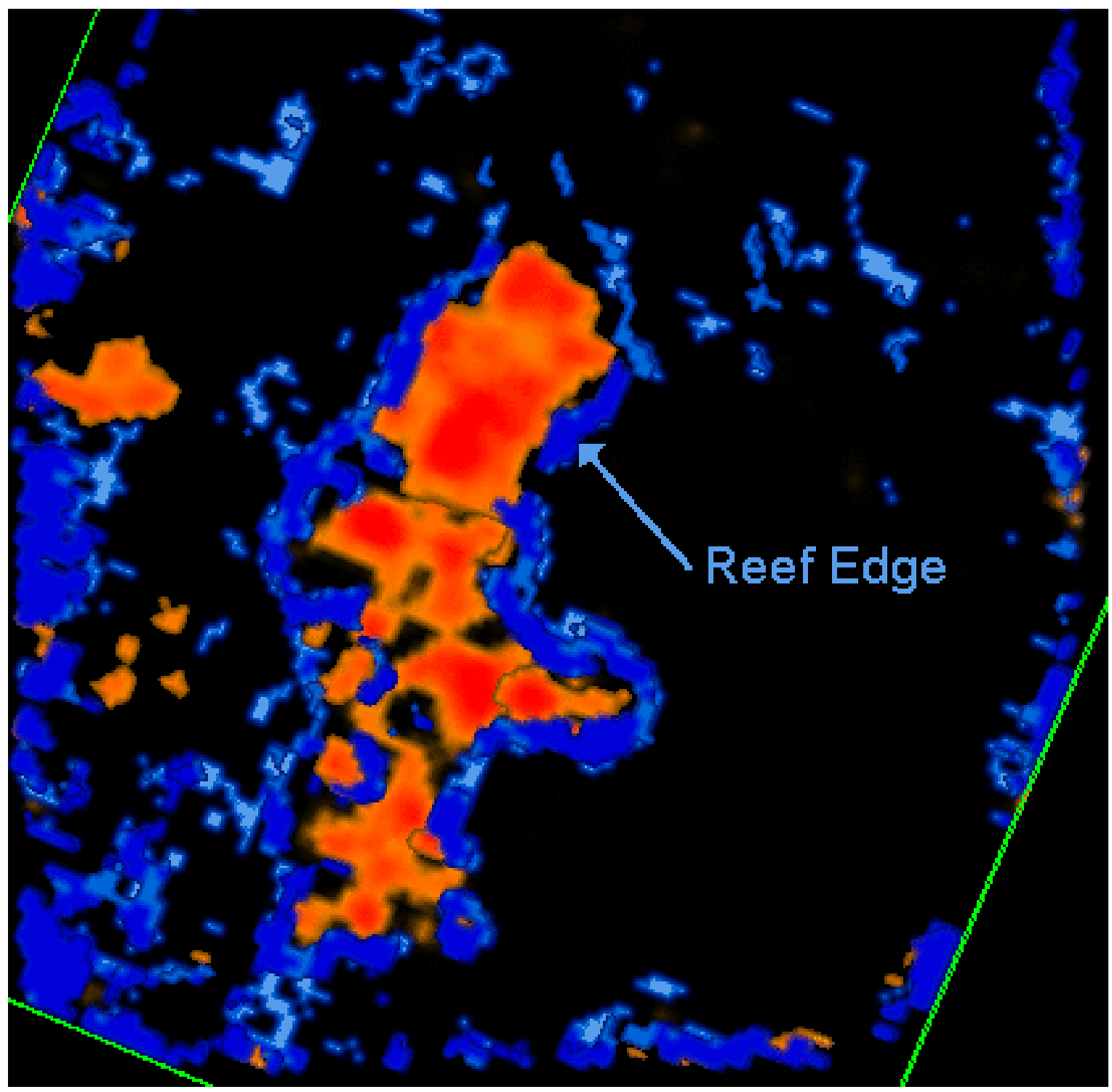

Figure 46: Blended seismic attribute time slab 855 to 860 msec. - high variance (in blue) and high amplitude (red-orange).

In addition to the seismic variance analysis an attempt was made to relate other seismic attributes to the porosity within the reef as measured with well logging data. Reflection magnitude 
and instantaneous frequency volumes were generated from the full azimuth seismic volume. In order to relate these attributes to the log derived porosity the seismic volume was examined in $2 \mathrm{~ms}$ intervals. Using the top and the base of the Guelph reef as a reference, which were constrained by the well to seismic ties, $2 \mathrm{~ms}$ time slices were taken through the attribute volumes. These time slices were then converted to depth using the time to depth relationships established during the well-toseismic ties, (see the first column in Figure 26). Figure 47 illustrates this technique.

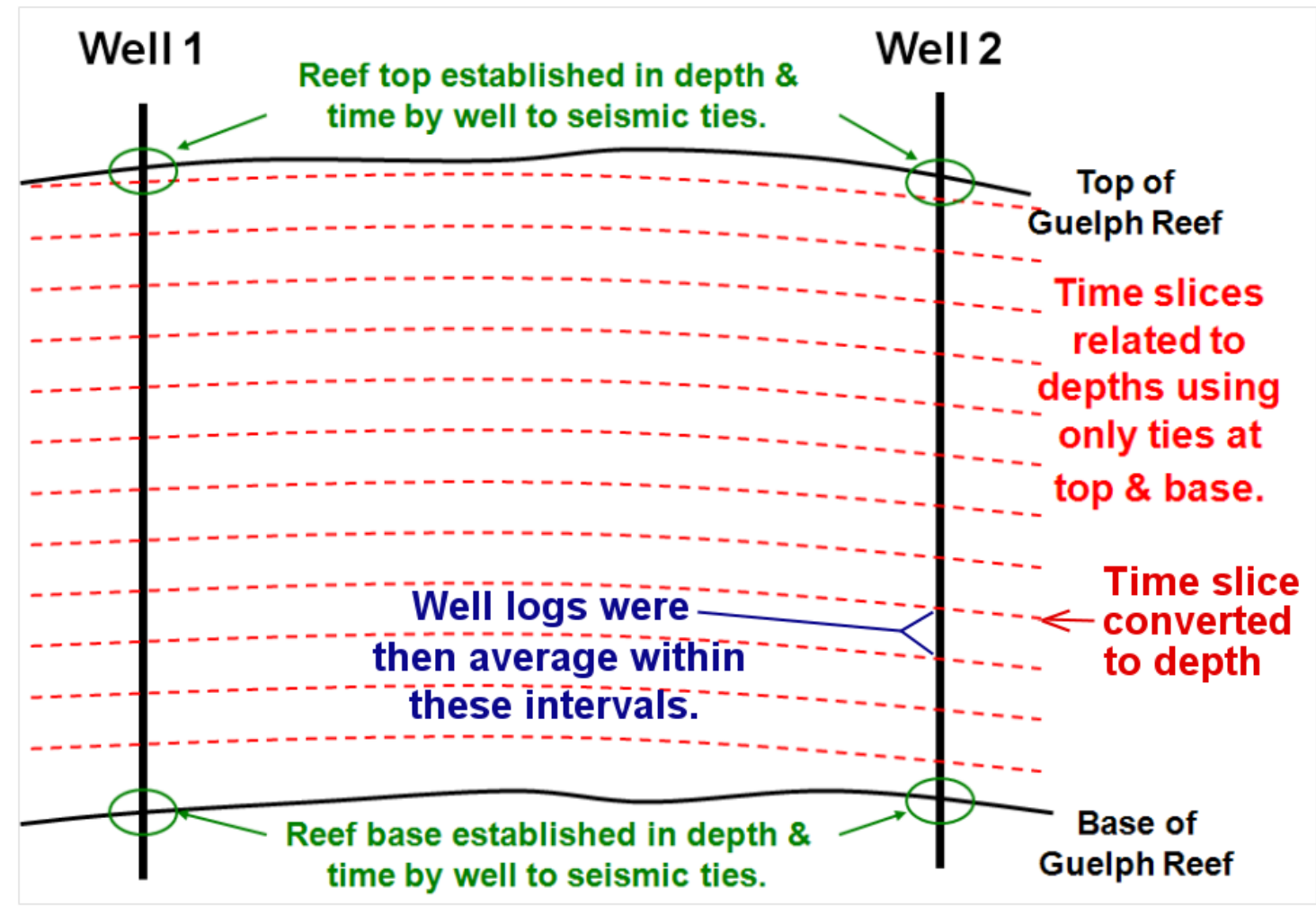

Figure 47: Illustration of method used for converting the $2 \mathrm{~ms}$ time slices to depth using the time to depth relationship established from the well to seismic ties.

This method allowed the determination of where in depth each time slice would intersect a specific borehole within the field. With this information I was able to determine the depths on each of the wells' neutron porosity logs that corresponded with each time slice. Neutron porosity logs for each well were then averaged between these depthed time slice intervals. Figure 48 shows the results of this process for two of the wells in the study area. The seismic attribute at the center of each zone was used in a comparison with the average log porosity value within the zone. As a result of this 
analysis it was determined that at best only a poor relationship was observable between reflection magnitude and the average porosity values.

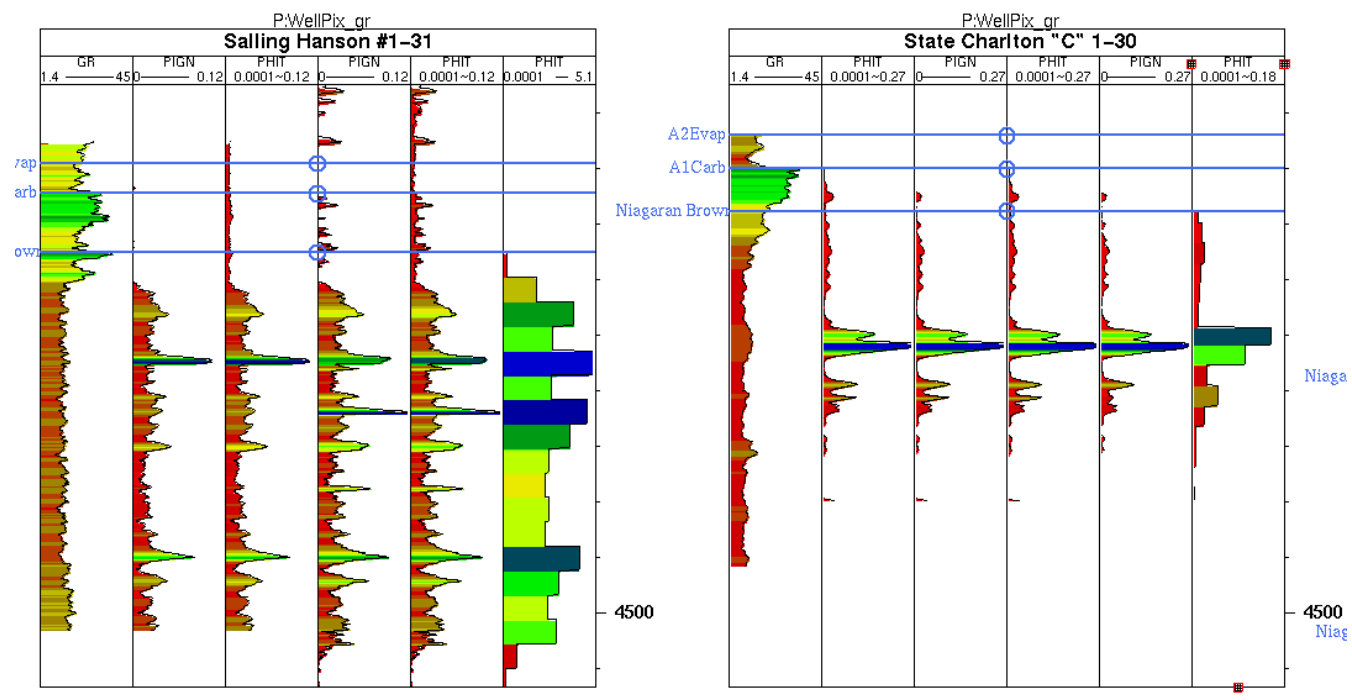

Figure 48: Gamma Ray, porosity logs and average porosity values between depthed 2 ms time slices for the Salling Hanson \#1-31 and state Charlton "C"1-30 wells.

However, a relatively good relationship was observed for the higher porosity values when compared with instantaneous frequency. This relationship was instrumental in helping to determine the porosity distribution within the reef and was instrumental in the development of the reservoir static model. This will be discussed in Chapter 4. 


\section{CHAPTER 4 - FREQUENCY ATTENUATION AND ITS RELATIONSHIP TO DRAINED, HIGH FLOW SYSTEMS}

\subsection{Low Frequency to High Porosity Relationship}

Once the basic structure of the reef was identified and mapped the investigation into the internal stratigraphy of the reef could begin. Seismic attribute analyses were again performed on the baseline 3-D volume to help identify internal stratigraphy. As mentioned in the previous section, Chapter 2, a number of different attributes were extracted from the volumes and examined. These attributes were compared to the porosity measurements obtained with the well log data.

Initially, time slices through the seismic volume at $2 \mathrm{~ms}$ intervals were converted to depth using velocities established during the well-to-seismic ties. Log porosity values were averaged within intervals of depth bounded by each $2 \mathrm{~ms}$ time slice. Figure 49 shows a correlation section through various wells in the field and displays the porosity $\log$ as recorded in track 1 and then blocked on the 2 msec intervals in track 2 .

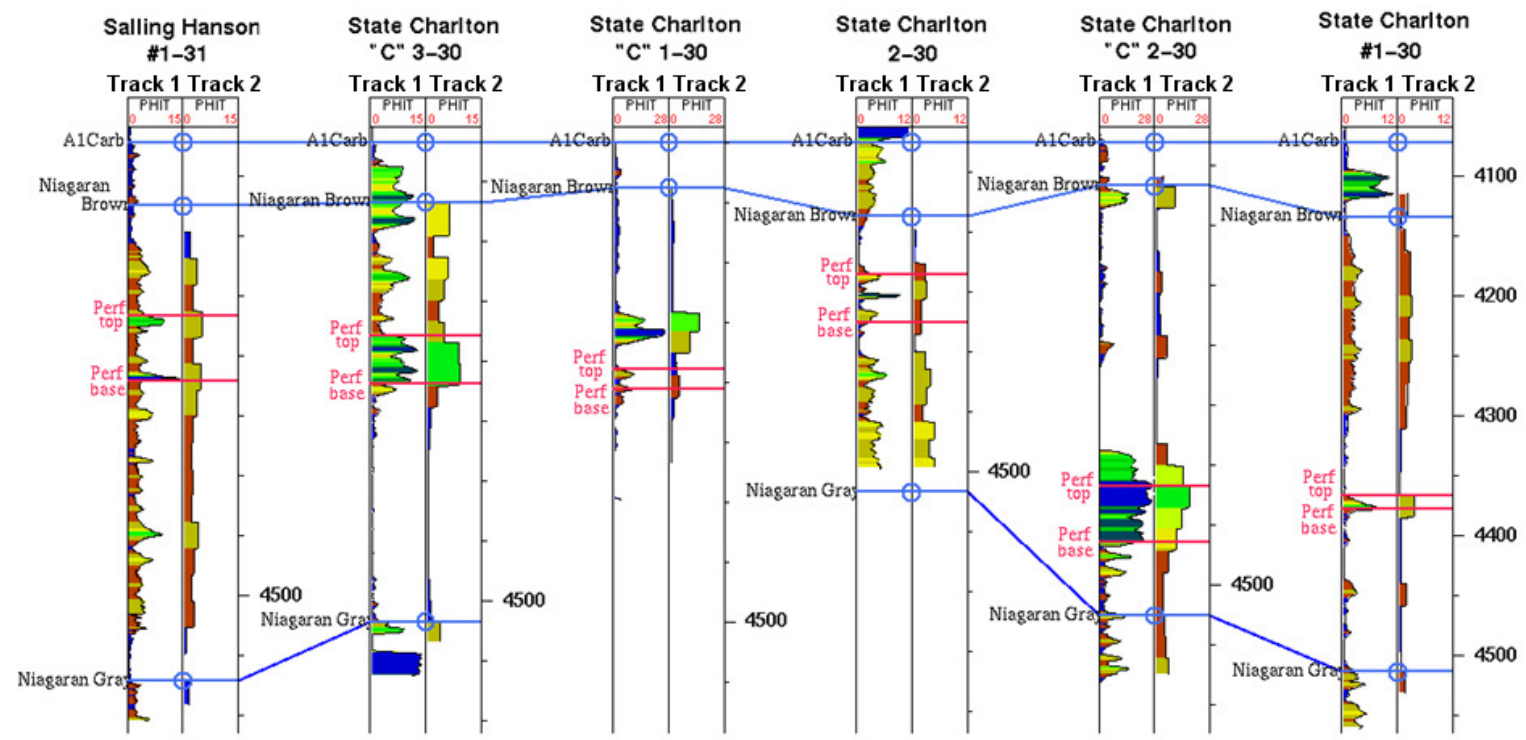

Figure 49: Original porosity logs (track 1 (left)) are plotted for comparison to the average porosity computed over intervals of depth corresponding to $2 \mathrm{msec}$ time intervals (track 2 9right) in each well $\log$ strip.

These averaged porosity log values were then compared with each of the seismic attributes that had been extracted from the seismic volume along the well bore locations. This analysis 
suggested a correlation between instantaneous frequency and porosity values greater than $5 \%$. This relationship is not well-defined but thought to be usable when characterizing the reef. Figure 50 illustrates this relationship for all data pairs within the reef. A "shotgun pattern" exists for all porosity values less than $5 \%$, indicating that all frequencies, high and low, are being returned by the lower porosity portion of the reservoir. However, a potential correlation can be seen for values above $5 \%$. The porosity / frequency pairs suggesting this negative relationship have been designated in red on Figure 50. The relationship indicated by the porosity / instantaneous frequency pair for porosity greater than $5 \%$ has a coefficient of determination of 0.3464 and correlation coefficient of -0.59 . The negative correlation is not a strong one, but is significantly negative. The $95 \%$ confidence limits on the slope fall between slopes of -0.47 to -3.92 about the mean of -2.194 . The low correlation is believed to be due in part to errors in the velocity function used to depth convert the data. Times of seismic events within the interior of the reef, when converted to depth, may not correspond precisely with the log values, see Figure 47.

The statistically significant negative relationship between increases of porosity and decreases in instantaneous frequency could have a significant impact on the characterization of this reef and, potentially many others in this trend. Additionally, this relationship might be extendable to other carbonate reservoirs around the world. To further this investigation a number of instantaneous frequency volumes were generated from the baseline 3D survey using various parameter settings. These 3D volumes were then compared to the well data. The results of this analysis are discussed in the next section 


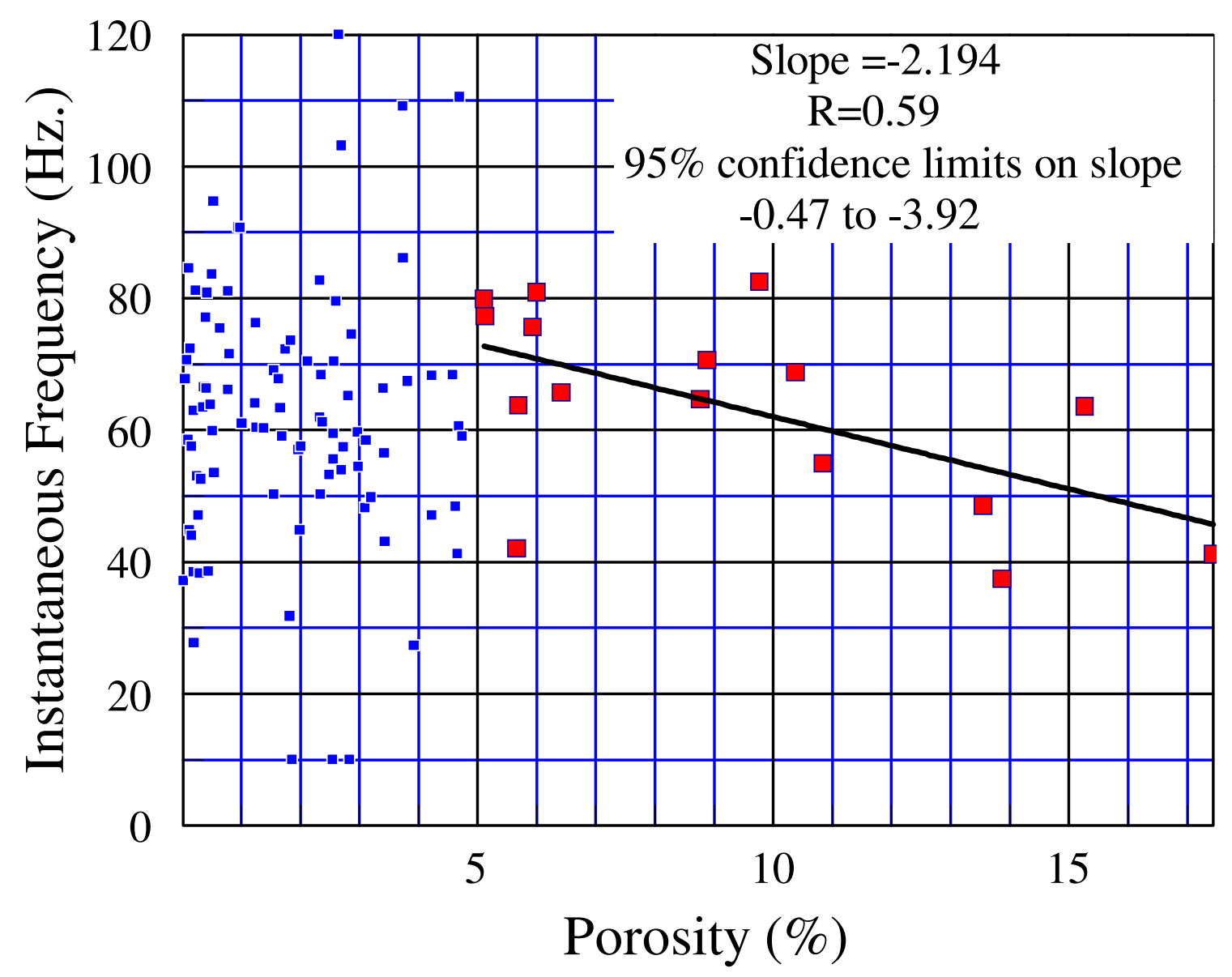

Figure 50: Blocked log porosity values used to compute an average porosity within reef for all wells is plotted versus instantaneous frequency observed in depth converted 2 msec time slices.

\subsection{Full-Azimuth Instantaneous Frequency}

An examination was conducted of the various instantaneous frequency volumes generated from the baseline survey. As shown in Figure 42 in Chapter 3 the frequency spectra extracted from various locations within the reef indicate that the low frequencies observed at these locations are between 10 and $18 \mathrm{~Hz}$ while the highest frequencies are between 105 and $115 \mathrm{~Hz}$. Instantaneous frequency volumes were generated using these different upper and lower limits as controlling parameters in the development of these instantaneous frequency volumes. After comparing the instantaneous frequency volumes the best one was selected for the porosity volume mapping based on the low frequency zone alignment with perforation zones. Figures 51, 52 and 53 show lines through one of these instantaneous frequency volumes that was determined to have the best 
correlation with the porosity logs during this comparison. These three instantaneous frequency sections are taken at the same locations as those shown in the previous section "Well-to-Seismic Tie Generation" (see Figures 32 to 38). In the following displays lower frequency corresponds to light blue.

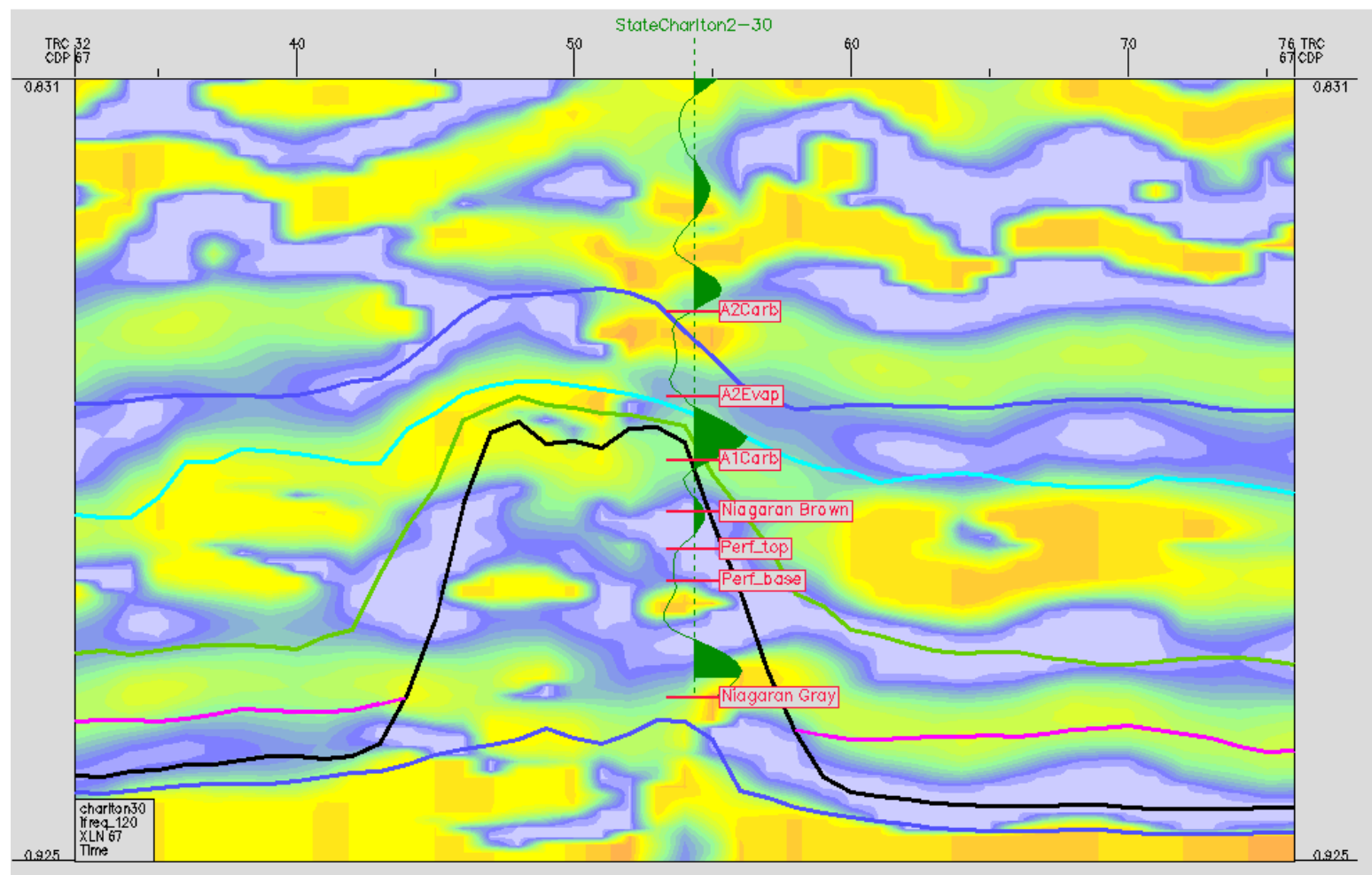

Figure 51: Instantaneous frequency display for the same line shown in Figure 34.

Examination of these instantaneous frequency displays immediately revealed that the intervals perforated during the well's primary production phase, designated "Perf_top" and "Perf_base" in these figures, all aligned with zones of low frequency. Since it is a normal industry practice to perforate in zones of high porosity this was taken as support for the relationship shown in Figure 50, namely that lower frequencies were associated with higher porosity zones identified in well $\log$ and later perforated in the reef. 


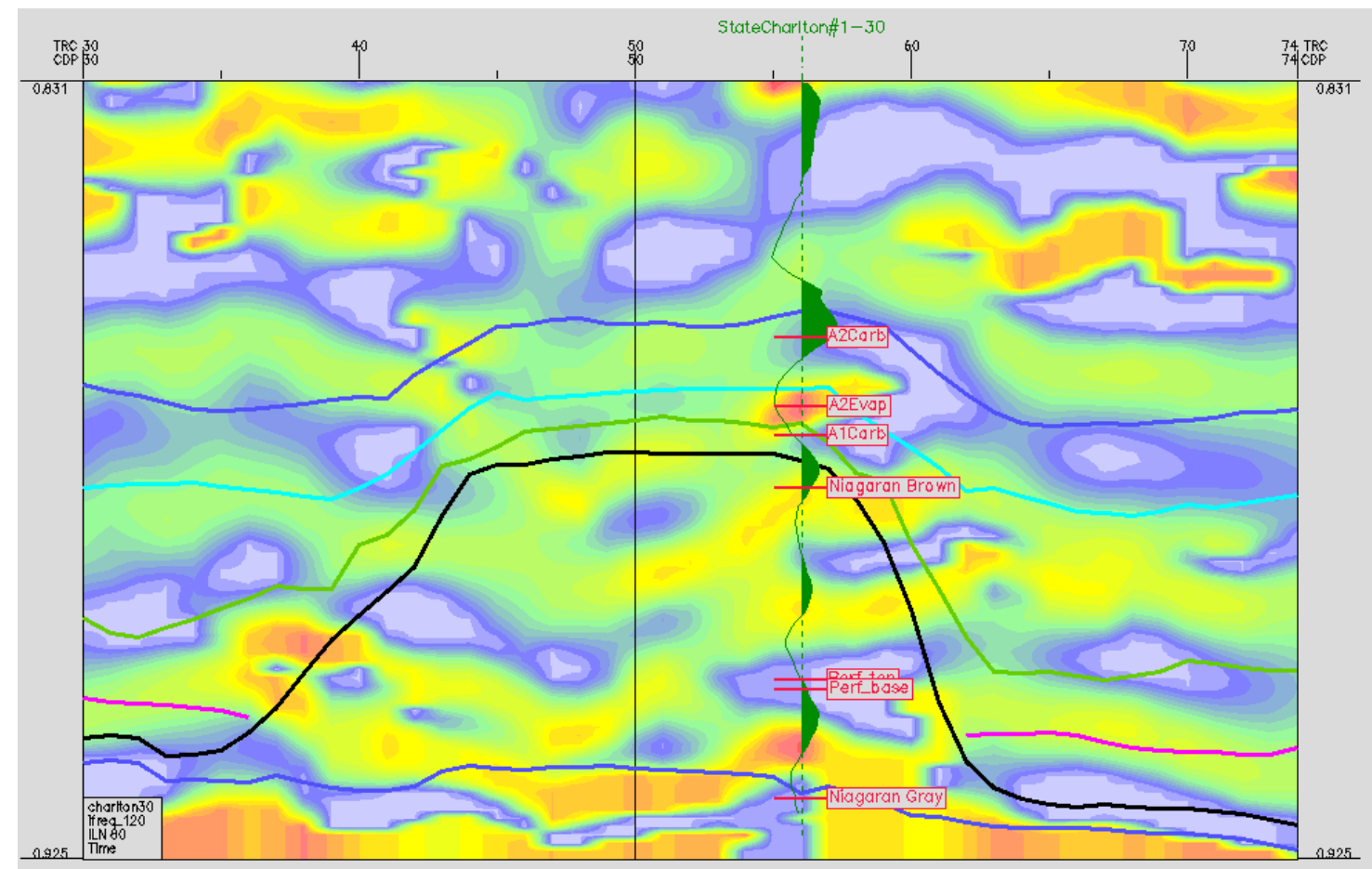

Figure 52: Instantaneous frequency display for the same line shown in Figure 36.

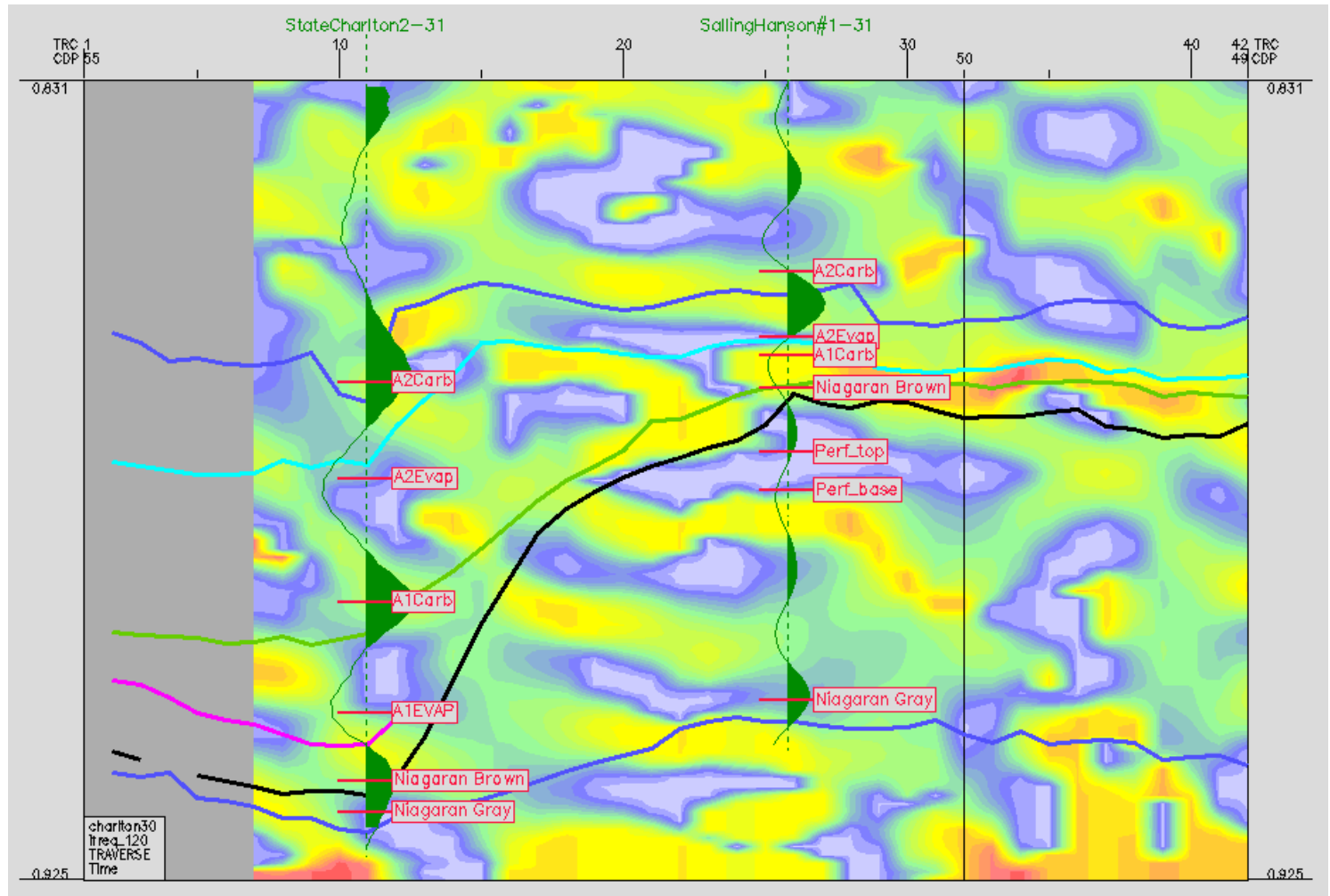

Figure 53: Instantaneous frequency display for the same line shown in Figure 38. 
The literature search reported in the "Background Information" section indicated that there was support in past publications for this relationship, specifically the relative strengthening of the lower frequency component of the signal as a result of the attenuation of higher frequencies due to wavelet induced fluid flow. This research relating frequency attenuation to high porosity / permeability, was initiated by Biot in the 1950s and dealt with the attenuation of higher frequencies due to fluid movement within porous zones. His papers, "Theory of Elasticity and Consolidation for a Porous Anisotropic Solid" (1955) and "Theory of Deformation of a Porous Viscoelastic Anisotropic Solid" (1956), both in the Journal of Applied Physics describe this phenomenon. An early effort to apply this work to the geosciences was performed by H. C. Misra in 1965 with the publication of a thesis entitled "Permeability of porous media to transient flow". In this work Misra theorized that "the permeability of the porous medium, as it occurs in the equations of motion, is frequency-dependent."

An initial attempt to use this relationship to generate a porosity volume for the reef for use in the reservoir characterization was attempted. Instantaneous frequency values from the time slices between the 2 millisecond bounding surfaces were used to influence the gridding of the log porosity values. Figure 54 shows the instantaneous frequency map on the right for one of the $2 \mathrm{msec}$ time slices, $889 \mathrm{msec}$, and the resulting porosity distribution map created using the frequencies as a guide on the left. 


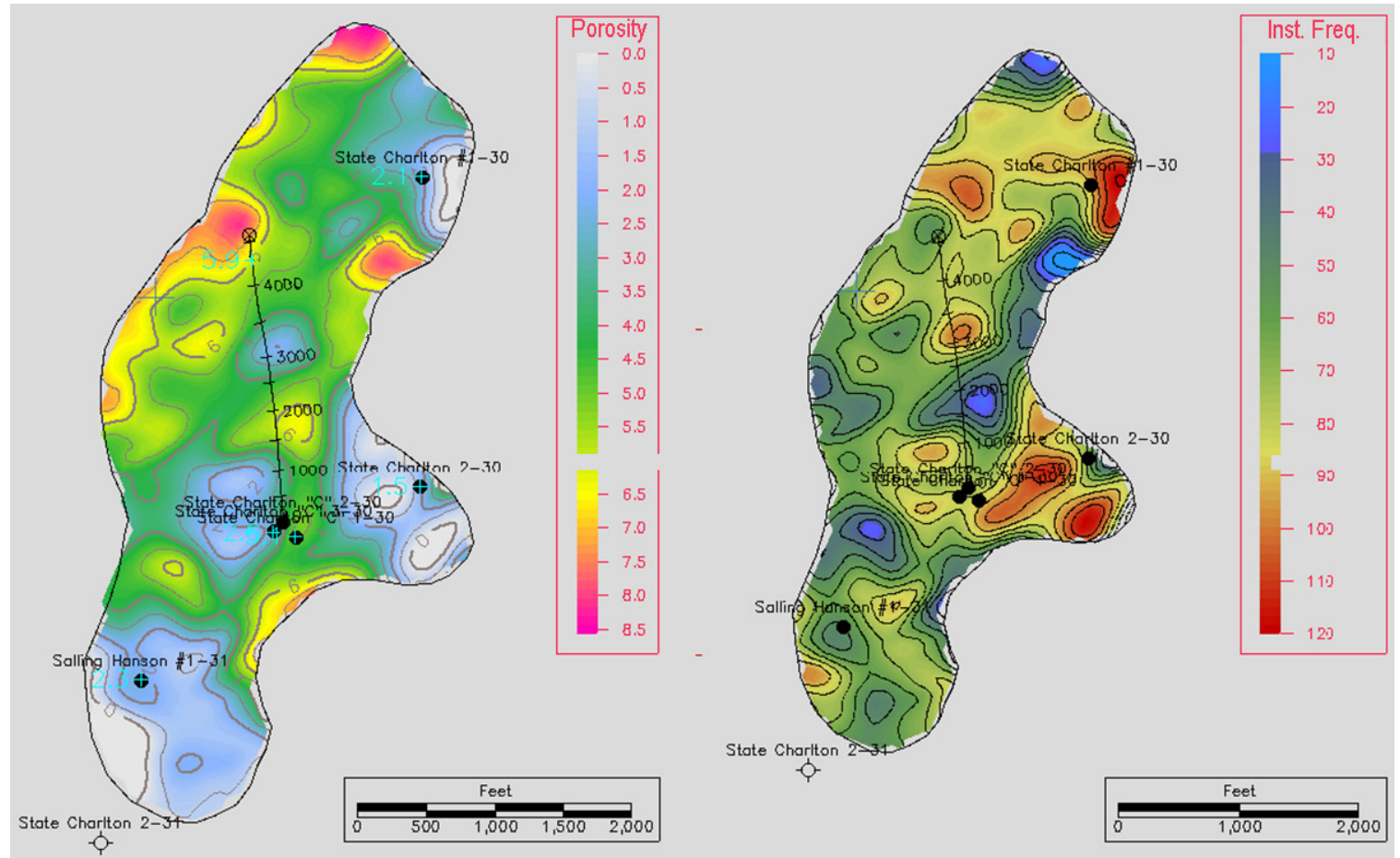

Figure 54: Porosity distribution map for time slice 889 (left) and the map of instantaneous frequencies (right) on which it was based.

Log porosity values for each time slice, shown in light blue on the porosity distribution map (left) of Figure 54 were also used to constrain the resulting surfaces. These 2 msec porosity distribution grids were used to construct a geologic model that was then used in an initial reservoir simulation of the field. The results from this initial simulation, which was based on the initial attempts to use gridded surfaces generated with the frequency - porosity relationship, indicated that the overall pore volume was too high but the porosity distribution was quite reasonable. The initial simulation based on the initial porosity distribution produced more oil than was actually produced from the field during the primary production phase. However, simulation also indicated that the percentage of the production relative to each well within the field was correct. Therefore the porosity distribution was acceptable and the overall porosity of bulk scaled slightly downward for use in subsequent simulations.

It was concluded that low frequency values, which were also being returned from the low porosity portion of the reservoir (see Figure 50), were pulling up the overall porosity in the 
simulation. This accounted for the pore volume being too high. However, the distribution of the porosity favorably corresponded with the well production history thus supporting the relationship.

\subsection{Porosity Distribution and Reservoir Characterization}

A second attempt was made to use this instantaneous frequency to characterize the reservoir's porosity distribution using a slightly different approach. The frequency spectra at additional locations within the seismic volume were investigated and the frequency range of the seismic in the vicinity of the reef was defined to a greater degree. These locations were selected in order to obtain a good distribution throughout the reef. The instantaneous frequency volume was then regenerated using this improved understanding of the reef's frequency signature based on the new frequency spectra to help set the parameters of the frequency extraction software. Figure 55 shows in-line 80 through the State Charlton \#1-30 well from the second instantaneous frequency volume. While this attribute section differs slightly from the same line shown in Figure 52, the frequency distribution is the same and the low frequency zones occur in the same locations. The main low instantaneous frequency zone, which occurs just above the lowest peak on the synthetic, is observed on both sections. Some changes in scale, expected due to the refinement of the frequency extraction parameters, are observable. At the center of the top of the reef a low frequency zone that can be seen in dark blue on the preliminary section (right) appears less intense (light blue green) in the more recent section (left). The frequency values in this area are the same in both volumes but the scale of the frequency range was expanded slightly during the generation of the second volume. 


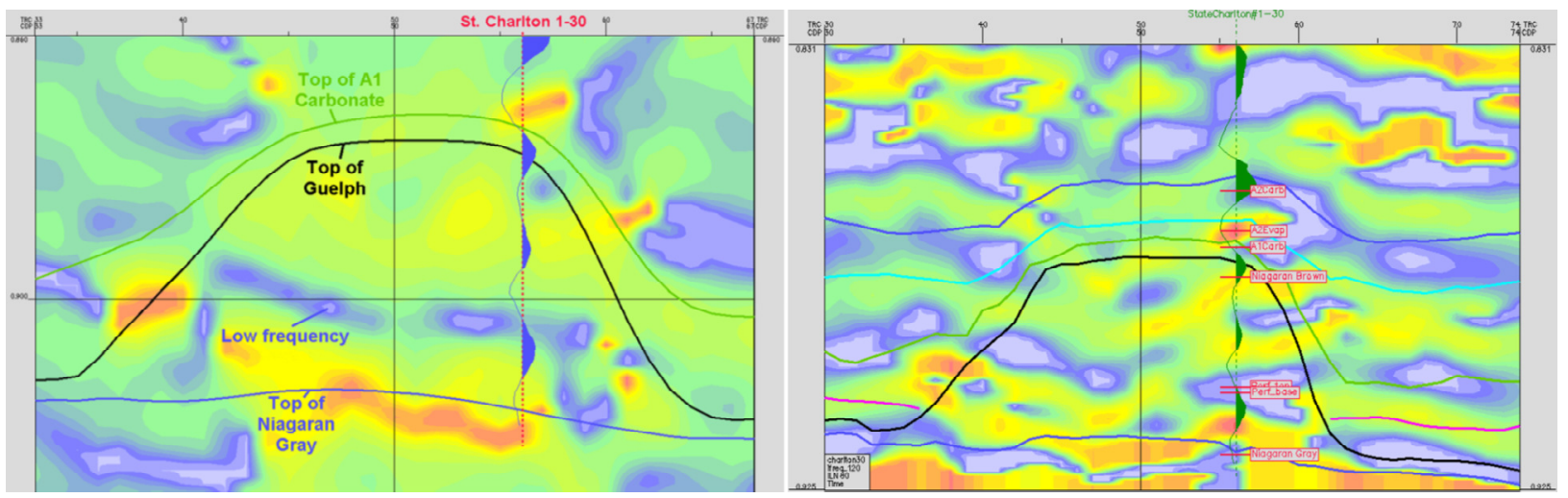

Figure 55: Instantaneous frequency display for In-line 80 through the State Charlton 1-30 well using the updated instantaneous frequency extraction parameters (on left) and the initial section (on right). Note that these screen captures are at different scales.

Using GeoFrame's IESX seismic interpretation application the instantaneous frequency volume was displayed and interpreted. The color map of the application's display was selected to help rapidly identify clusters of lower frequency within the seismic volume. The tops and bases of these low frequency zones were interpreted. This interpretation, along with the instantaneous frequency volume, was then transferred into the static model construction program Petrel. Within this program the porosity to frequency relationship shown in Figure 50 was applied to the volume within the low frequency clusters. Within these zones the porosity distribution was set to greater than $5 \%$ and propagated throughout these subvolumes using the relationship, $y=-2.194 x+83.965$ and guided by the actual instantaneous frequency values. This was a deterministic methodology that was based on the seismic data. Outside of these low instantaneous frequency subvolumes a distribution of 1 to $5 \%$ porosity was propagated throughout the rest of the reef using stochastic methods. The resulting static model was a combined or "blended" deterministic / stochastic static model.

This second porosity volume was believed to be more finely tuned than the original one due to the refinement of the frequency range found by extracting the frequency spectra at additional locations within the seismic volume. This new volume was then used in the creation of a new geologic model and reservoir simulation. This new porosity distribution proved to be a very good 
starting point for the history matching process. Areas around four of the six production wells required little or no adjustment to create a reasonable history match. The remaining two wells, clearly showed production interference effects between them. This was determined due to the rapid response of pressure and production in one well once the other came on line. This required additional history matching efforts within the area of this two wells as they were producing more oil as indicated by the simulation than was actually produced. The main adjustment performed to the model was the regional (bulk) reduction of the porosity array in that area.

\subsection{Dump Flood}

As the principal investigator for the Department of Energy research project I oversaw work performed by other members of my research team. This included work performed by my team's reservoir engineer. During the early phases of the project reservoir engineering data suggested that a dump flood had inadvertently occurred within the field. During the well remediation phase, it became apparent that pore pressure in the reef was much higher than the estimated 1997 abandonment pressure of 500 psia. In December 2004 a bottomhole pressure (BHP) gauge recorded over 1,700 psi. Several other BHP measurements confirmed the anomalous high formation pressure and an explanation was sought.

The well remediation program revealed a serious condition common to all the wells, corroded casing. In the case of the Charlton 1-31 and Charlton 2-30 wells, casing leaks were severe and water was entering the wellbores from shallower formations, particularly the Dundee Formation. Locally, the Dundee (see Figures 25 and 27) is used as a produced water disposal zone for other projects. It is unknown when the inadvertent injection, or "dump flooding" of water began in the reef, how many injection points there were, and what the cumulative volume injected was.

Discovery of the dump flood has helped explain the reported increase of produced water from the Charlton 2-30 well in 1985 and the sudden arrival of 100\% water cut in the "C"2-30 well in 1997, 
which ended primary production from the field. 2004 remediation work on the "C" $2-30$ and Charlton 1-30 wells did not find any casing leaks across the Dundee Formation, but the condition of the casing in the abandoned "C"1-30 and "C"3-30 wells is unknown.

The dump flood, and the limited understanding of it, has been an important complicating factor in forward planning and execution of the $\mathrm{CO}_{2}$ flood. Simulation efforts replicated the repressuring of the reef with dumpflood water. An estimated 2.3 million barrels of dump flood water have been added to the reef system between 1985 and 2005, at which time known casing leaks were repaired.

\subsection{Reservoir Simulation}

\subsubsection{Production History Matching}

The simulation was designed to take maximum advantage of the high resolution 3-D seismic survey. The simulation grid was laid out parallel to the seismic lines and cell size was set at 82.5 foot squares, the same as the bin spacing of the seismic data. Also, grid cell thickness was set at 9 feet, equivalent to the seismic sample rate of 1 millisecond through the reef.

This effectively created one simulation grid cell for each seismic sample point, with the effect of eliminating the need for upscaling of the seismically derived data as the static model grid cells were equal to the distance between the seismic samples and the reservoir simulation grid was created with the same spacing. The resulting simulation grid contained 48 x 87 x 29 cells. Within the reef isopach, there is no easily identifiable layered stratigraphy that might be considered as coherent flow units. Partial dolomitzation of the reef limestone creates much of the storage capacity and most of the permeability in the reef. However, the seismic derived porosity appears to be distributed into horizontally extensive intervals through the reef (Figure 56) and the use of a uniform horizontal layering configuration in the simulator was able to sufficiently represent reef's lithologic variation. The resulting grid structure with the seismically derived porosity distribution is shown in Figure 56. 


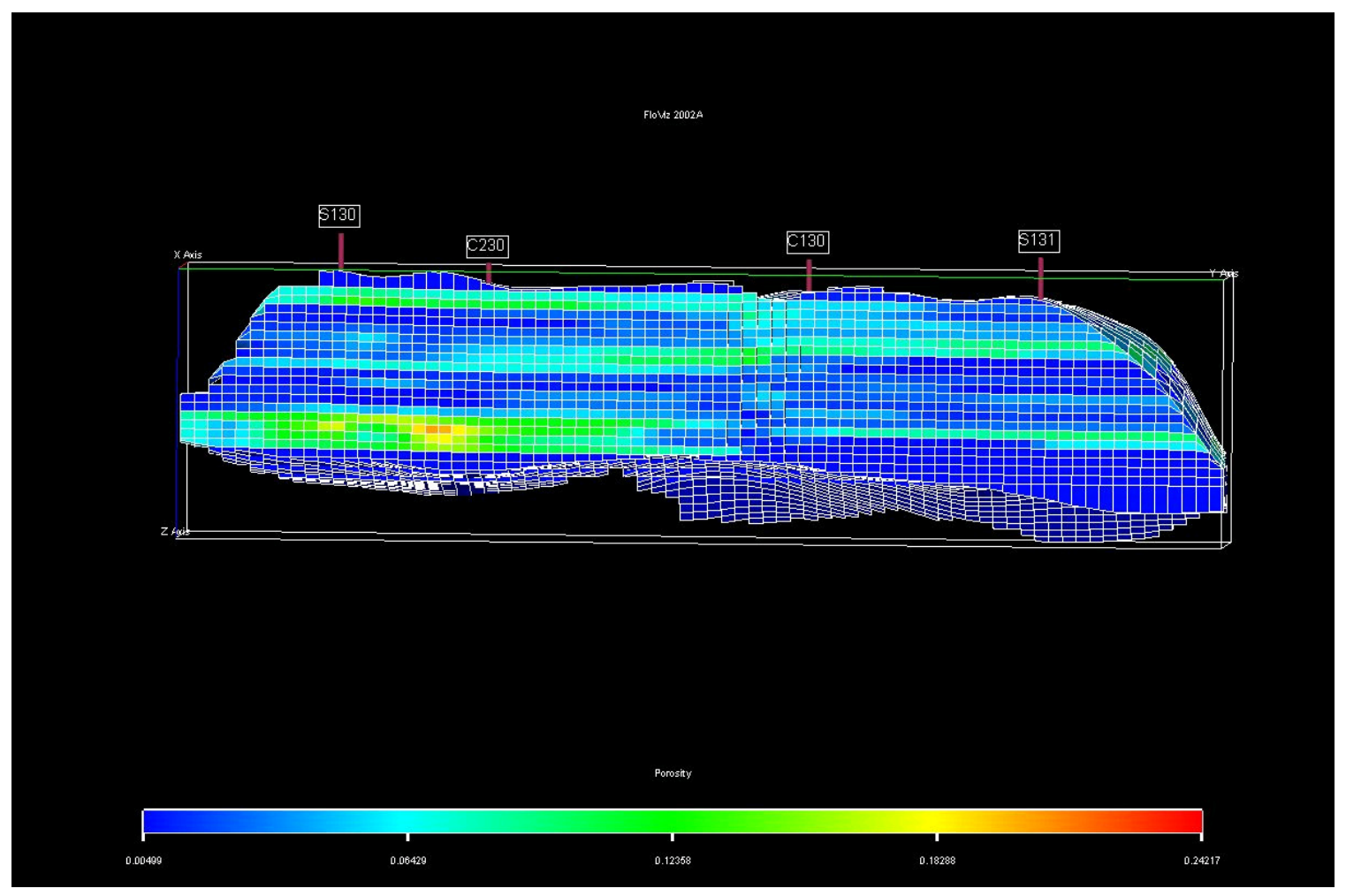

Figure 56: Cross section of simulation showing the distribution of reservoir grid cells in the model and seismic based porosity distribution.

History matching was accomplished using the seismically derived static model developed with the relationship shown in Figure 50 with relatively few modifications. This history match is shown in Figure 57. The overall pore volume from the static model was reduced by about $20 \%$ to achieve the observed field pressure decline, but the field porosity distribution was retained with only one exception. The exception area was between the State Charlton "C"2-30 and State Charlton 1-30 wells which have showed clear interference effects between them during depletion, pre- $\mathrm{CO}_{2}$ flood testing and during early $\mathrm{CO}_{2}$ flood operations. Additional pore volume reduction between these wells was required to match the effects seen between these two wells. This observation was to have significance later in the DOE project and will be discussed in more detail in a later section. 


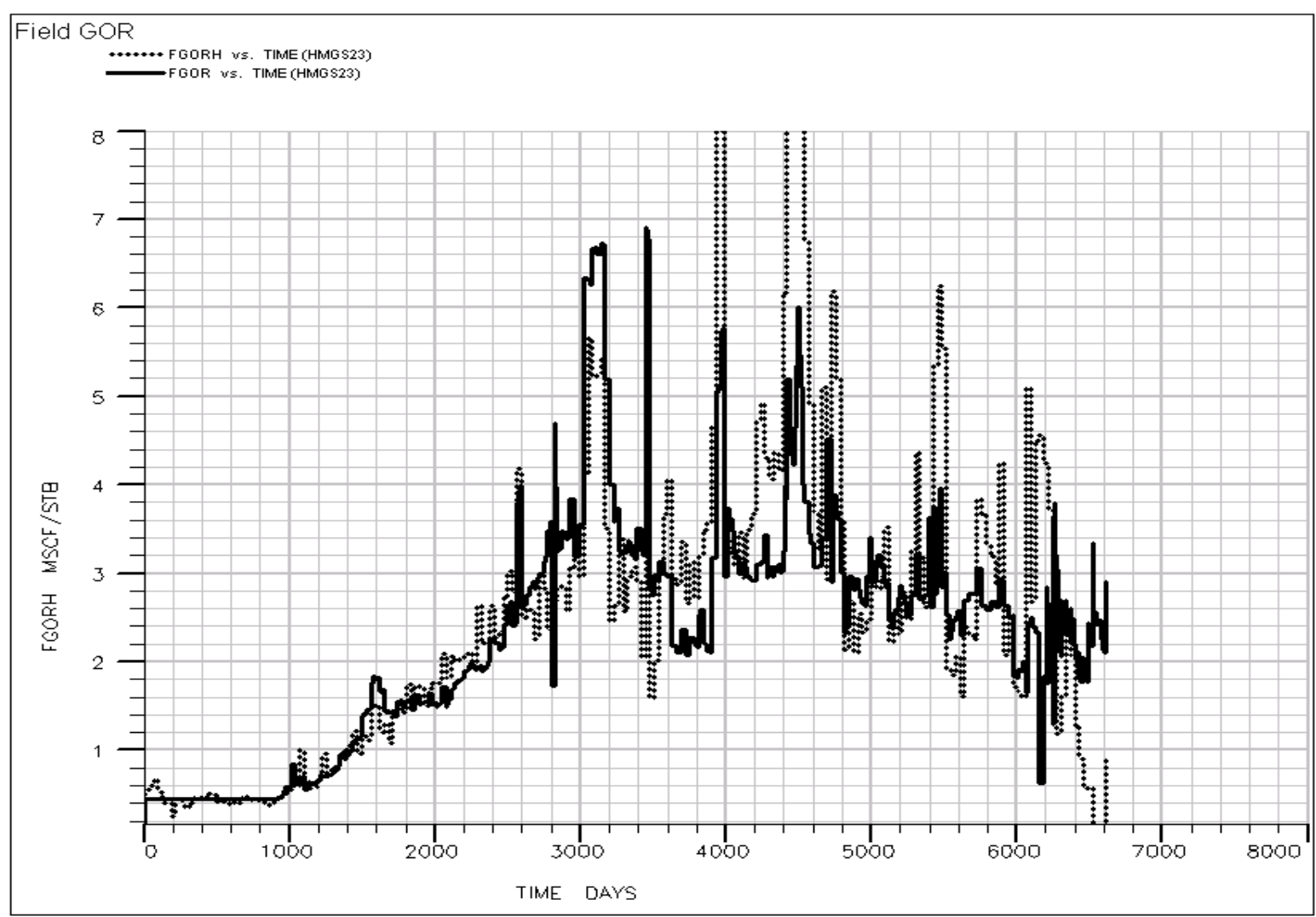

Figure 57: History match showing 18 years of field GOR (Gas to Oil Ratio) history, dashed line, and simulated GOR, solid line.

The results of the history matched reservoir simulation support the relationship suggested in Figure 50. As noted previously a relationship exists between lower instantaneous frequency and higher porosity when porosity values are greater than 5\%. Unfortunately, lower instantaneous frequencies can also be found in rocks with less than $5 \%$ porosity. When the relationship shown in Figure 50 is applied to the entire reef, rocks with porosities lower than $5 \%$ are artificially boosted to higher porosity values, and this somewhat optimistic estimation of pore volume was confirmed by the need to bulk reduce pore volume in the simulation. Figure 58 below illustrates how this concept works. 

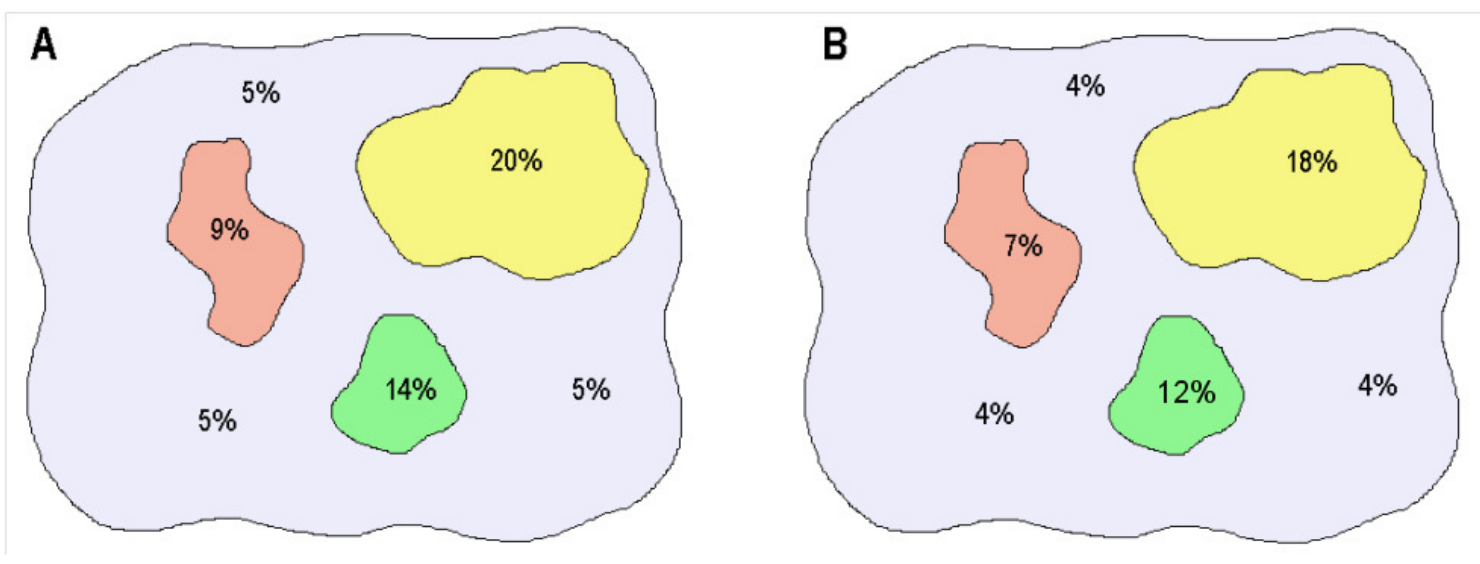

Figure 58: Porosity configuration shown at left has its overall bulk porosity decreased in the display on right but the distribution of the porosity zones remains the same.

The period of the dump flood also had to be "history matched". This was the time between the end of primary production in 1997 and the start of the 2004 well remediation program. The bottomhole pressures recorded in 2004 and 2005 confirmed that pore pressure in the reef was in the range of 1,600 to 1,800 psi. This roughly coincides to the height of a column of salt water between the reef perforations and the source Dundee Formation. Therefore, the dump flood was replicated in the simulator by introducing constant pressure water injectors at the wells with known casing leaks. This injected 2.3 million barrels of water into the simulated reservoir and repressured it to 1,650 psi.

After repressuring, the history matched model was used to create a variety of $\mathrm{CO}_{2}$ flood development scenarios. The simulator was constructed using black oil PVT data, which is the Pressure, Volume, Temperature data for non-volatile rich (black) oil from the reef. Coats (1995) give a good review of the use of this type of data as well as for the compositional (volatile rich) models. Additionally, the $\mathrm{CO}_{2}$ injection was handled by using the 4-component solvent model methodology with $\mathrm{CO}_{2}$ as the solvent. The Todd-Longstaff miscible fluid mixing parameter technique (Todd, 1972) was applied. The most advantageous $\mathrm{CO}_{2}$ flood development plan was not immediately apparent. Several factors considered in the planning, included:

1) The highly irregular yet continuous high porosity and permeability distribution due to dolomitization. 
2) The thick oil column, over 300 feet, and the apparent gravity drainage effects seen during depletion.

3) The elongate shape of the reef structure.

4) The limited number of wellbores to create an effective injection/ production pattern.

The most effective simulation scenario involved injection into the two most northerly wells, sweeping oil to the southern wells. Core Energy ultimately adopted a variation of this scenario by initially injecting $\mathrm{CO}_{2}$ only into the second most northerly well, the "C"2-30. During this initial phase of $\mathrm{CO}_{2}$ injection, production from the Charlton 1-30 (the northernmost well) continued (see Figure 19 in Chapter 3). The intent was that after approximately one year, when the Charlton 1-30 was expected to begin cycling unacceptable amounts of $\mathrm{CO}_{2}$, it would then be converted to injection to push remaining oil toward the southern producers.

\subsubsection{Forward Predictive Model}

The production history match performed on the initial reservoir simulation validated the porosity and permeability distribution within the reservoir. Permeability to Porosity relationship was established with data from pre-existing core reports. This reservoir simulation used the oil, gas and water production rates reported for the wells over the primary production phase of the Charlton 30/31 field, which was from 1974 to 1997. The actual production rates reported strongly correlated with those predicted by the reservoir simulation. An example history match is shown in Figure 57.

The validated model served as the basis for a number of predictive, forward-looking reservoir simulations developed to model various EOR scenarios. These models were performed to investigate different potential injection / withdrawal configurations within the field. Existing well locations as well as new well locations were investigated as $\mathrm{CO}_{2}$ injectors and EOR producers in order to select the optimum flooding configuration. With the selection of the State Charlton "C" 2-30 well as the $\mathrm{CO}_{2}$ injector and the State Charlton \#1-30 well as the EOR producer a final predictive reservoir simulation was made to forward model the field's response using this scenario. This dynamic model, 
like the static model on which it was based, was composed of various layers of three-dimensional cells. These layers are typically referred to as "K slices". Various reservoir dynamic attributes, such as oil saturation, $\mathrm{CO}_{2}$ saturation or water saturation, can be depicted within the cells of these $\mathrm{K}$ slices at various time increments. These time increments are generally referred to as "time steps".

By the summer of 2007 time constraints for the Department of Energy project mandated the acquisition of the second or "Monitor" 3-D survey. This survey was acquired in September of 2007. Before the acquisition of the "Monitor" 3D survey a predictive, forward looking reservoir simulation was develop that was based on the initial reservoir characterization. It should be noted that, as with the reservoir simulations developed for EOR operation optimization, this reservoir characterization was developed using the "Baseline" 3D survey and the method previously described in this chapter's section entitled "Porosity Distribution and Reservoir Characterization". This specific reservoir simulation differed from the previous ones in that it used the actual (instead of planned) $\mathrm{CO}_{2}$ injection rates and pressures that had occurred during the field operations up until the summer of 2007. This forward-looking simulation was developed in order to predict the location of the injected $\mathrm{CO}_{2}$ within the reservoir at the time the "Monitor" 3D survey was acquired. Should the simulation results compare favorably with the results of the $4 \mathrm{D}$ survey it would be another indication that the reservoir characterization, which was based on the relationship shown in Figure 50, was correct. Figure 59 the distribution of oil within K (permeability) slice \#9 at times step September 1, 2007. This slice correlates with the upper portion of the reservoir and is used later in the study to relate features observed in the Monitor survey to this simulation. Figure 60 shows the distribution of $\mathrm{CO}_{2}$ within the same slice and time step. 

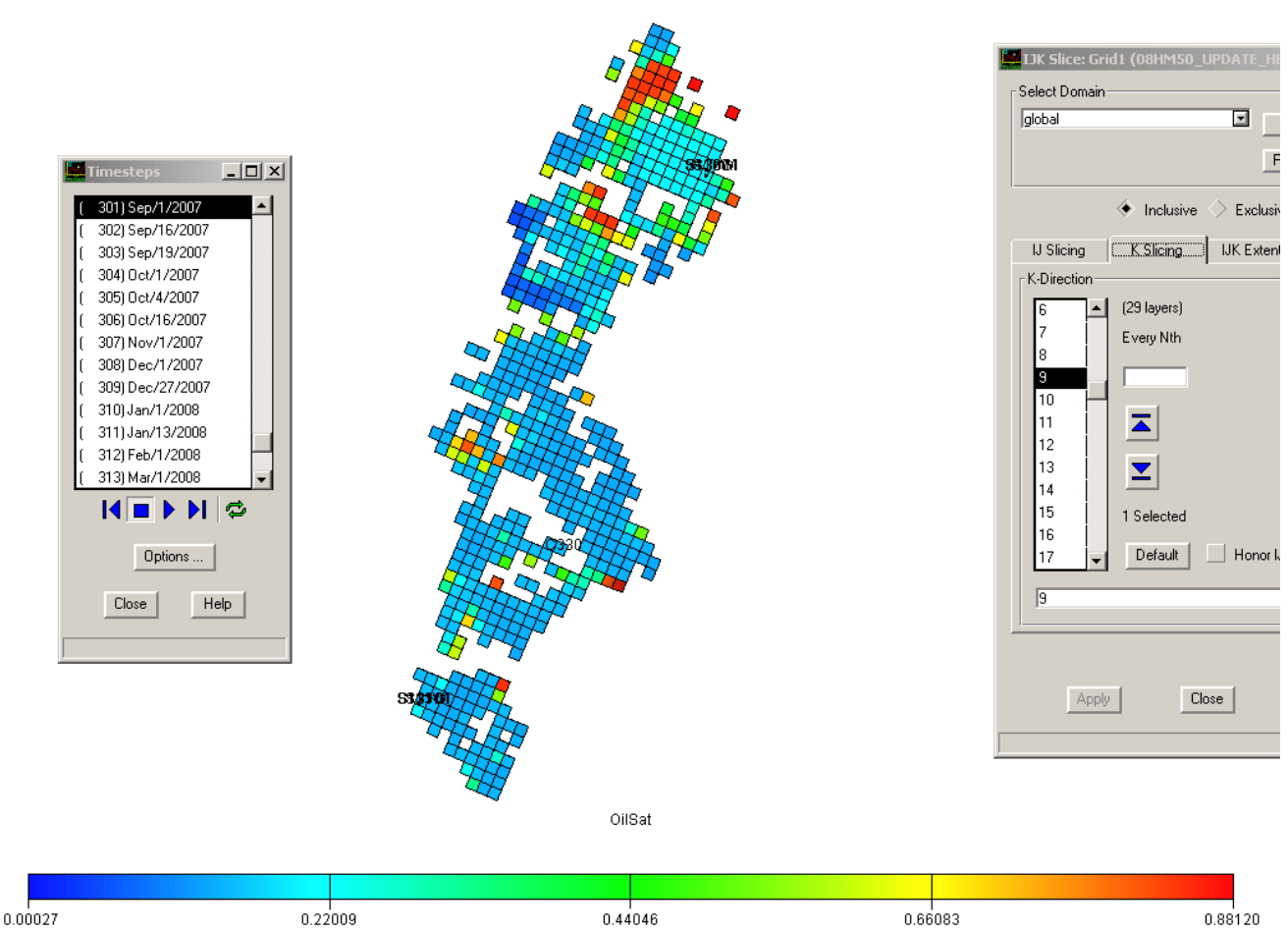

Figure 59: Predicted oil distribution within the Charlton 30/31 reef for $\mathrm{K}$ slice 9 at time step September 1, 2007 from the predictive, forward-looking reservoir simulation.

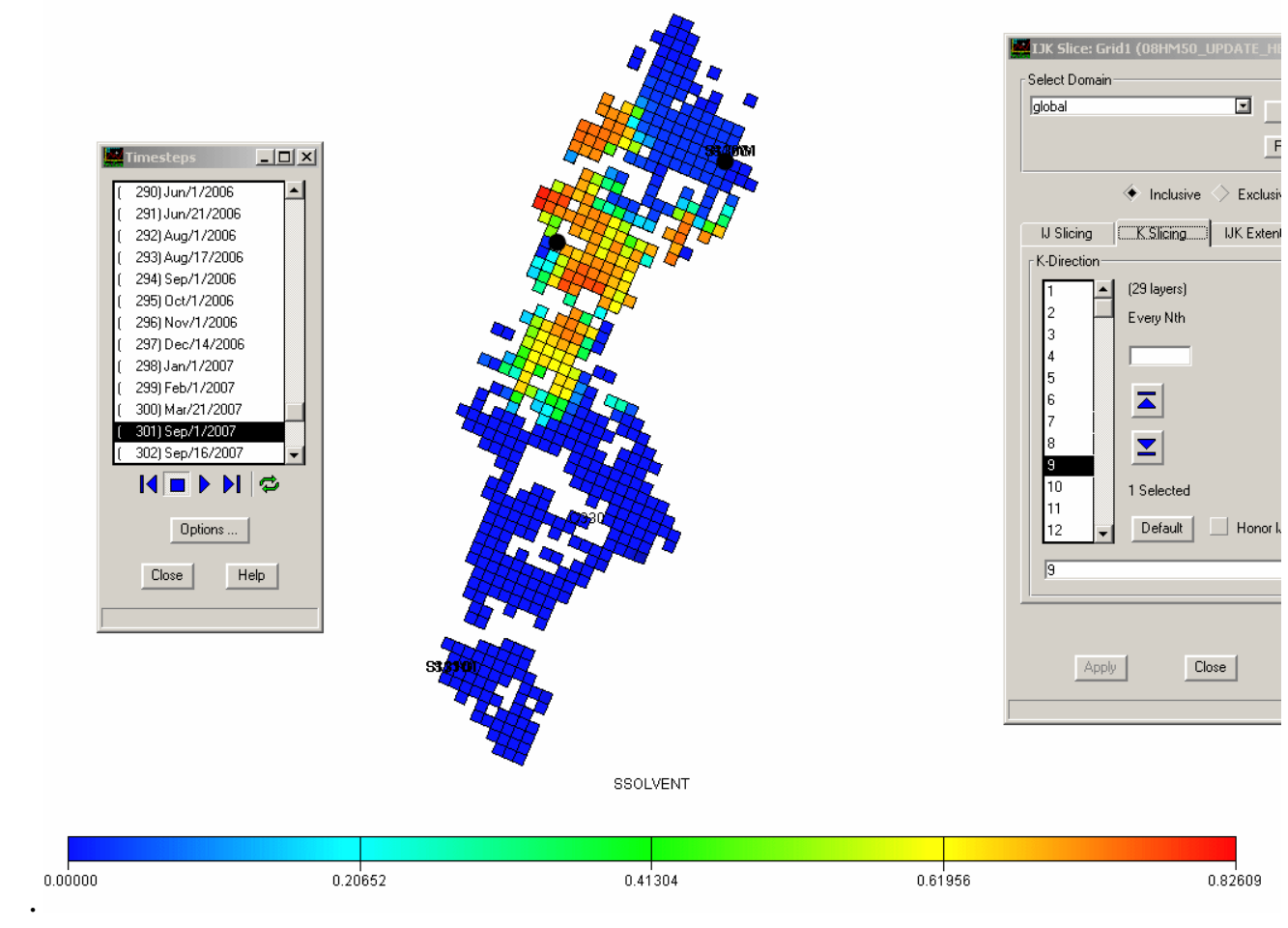

Figure 60: Predicted CO2 saturation distribution within the Charlton 30/31 reef for $\mathrm{K}$ slice 9 at times step September 1, 2007 from the predictive, forward-looking reservoir simulation. 
The results of the predictive, forward-looking reservoir simulation for September 2007 were compared with the results of the analysis of the 4D seismic survey. These results are reported in the next section, "4D Seismic Analysis".

\subsection{D Seismic Analysis}

After the Monitor 3-D survey was acquired in September of 2007 it was possible to generate the Charlton 30/31 4D seismic survey. Raw seismic data acquired for the Baseline and the Monitor surveys were sent to WesternGeco for time lapse or 4D processing. Upon completion of the 4D processing both data sets, the 3D Baseline Survey and the 3D Monitor Survey, were loaded into a geophysical workstation. Additionally, all available well data previously used in this project was loaded into this new workstation project. Wavelet analysis and well to seismic ties were performed on both of these surveys and then compared with those generated for the pre-injection (Baseline) volume using the original processing flow (see section 3.3.3 in Chapter 3).

The seismic signature of the reef on the Baseline 3D survey matched the Monitor 3D in character. The on-reef section is composed of four peak events from the top of the A2-Carbonate to the top of the Niagaran Gray. The seismic signature can be seen in both processing sequences and is confirmed by the well-to-seismic ties. As noted during the investigation of the "Baseline" 3D, reported in Chapter 3, under the "Well-to-Seismic Tie Generation" portion of section "Initial Structural and Stratigraphic Investigations", the off-reef section is composed of only three peak events from the top of the A2-Carbonate to the top of the Niagaran Gray on both sequences, whereas the on-reef is composed of 4 peaks (see Figure 36). The change in seismic response is the result of the $90 \%$ decrease in the thickness of the Guelph formation as the pinnacle reef facies (approximately 380 feet thick) to the non-reef facies. This same seismic signature can be seen on the original 3D processing as well as the 3D volumes created during the 4D processing. Additional investigation 
confirmed that the original horizon interpretation performed on the original "Baseline" 3D data set (processed by itself before the acquisition of the "Monitor" survey) matched the 4D seismic processing version (which used both "Baseline" and "Monitor" surveys during processing) quite well. Some refinements to the interpretation had to be performed but these adjustments were minor.

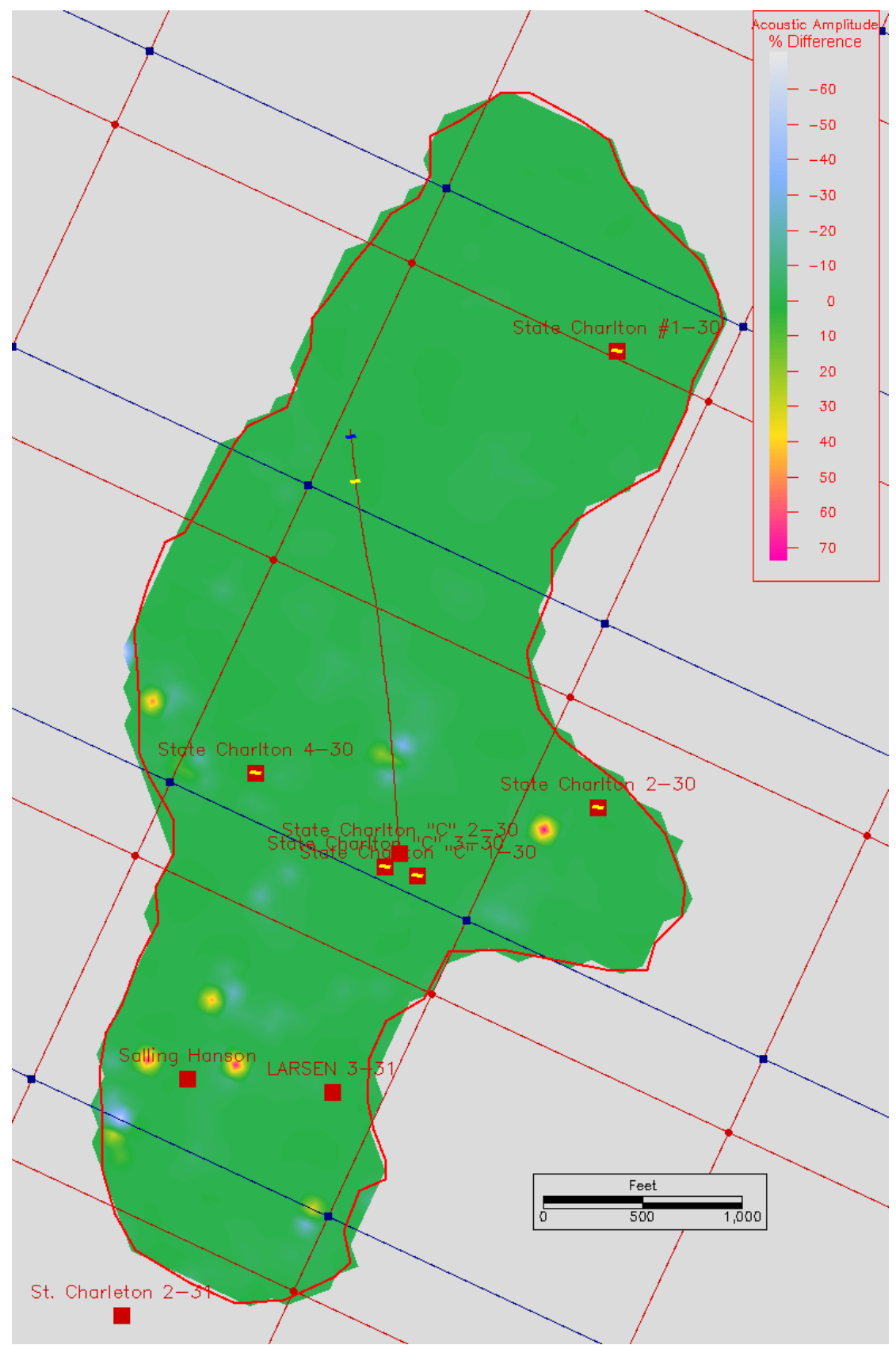

Figure 61: Percent amplitude difference for A2-Carbonate between Monitor and Baseline surveys. 
Since both the Baseline and Monitor 4-D surveys were developed with the exact same processing sequence during the 4-D processing, significant variations in amplitude between the two volumes due to processing effects were not expected. A comparison of these two surveys is shown later in this chapter. As shown in Figure 61 and later in this chapter some amplitude differences were observed in the southern portion of the survey. One possible source of amplitude variation between the two surveys was believed to be due to the differences in the time of year when the surveys were acquired, as discussed previously. Another possible source of amplitude variation is minor change in acquisition geometry. The monitor survey was not extended as far to the south because reservoir simulations of the $\mathrm{CO}_{2}$ injection, based on the original Baseline 4D survey, indicated that the $\mathrm{CO}_{2}$ would be concentrated within the northern end of the reef. Additionally, some minor changes in the source and receiver geometry were necessary for the Monitor survey due to licensing and landowner restrictions. A few landowners restricted access to their lands for the second 3D survey. These, however, were kept to a minimum and their effect believed to be negligible. As can be seen in Figure 61 these effects were limited to the southern end of the survey, away from where the EOR operations.

In order to determine the variations in signal strength that might be due to these minor changes in acquisition geometry, a strong reflection event from a non-reservoir stratigraphic unit was selected for interpretation that was within the immediate vicinity of the reef. The A2-Carbonate was selected for this comparison. This stratigraphic unit is the strongest reflector immediately above the reef and is easily recognizable across the area (see Figure 18 in Chapter 3). This event was easily and rapidly interpreted on both the Baseline and Monitor 4-D surveys.

The amplitude of the A2-Carbonate peak event was mapped for both surveys. The amplitude ranges for the area directly above the reef were compared on both surveys. It was found that the amplitude variation was extremely similar in range and distribution as indicated by Figure 61. For the Baseline survey the A2-Carbonate amplitudes ranged from -155 to 4529. For the Monitor survey 
the A2-Carbonate amplitude ranged from -34 to 4691. In order to bring these two surveys into a closer alignment for comparison purposes the A2-Carbonate amplitude was gridded for the monitor survey and scaled by .99 and biased by -120 . This produced an amplitude range of -153 to 4524 . This adjustment brought the amplitude range for the A2-Carbonate into alignment for both surveys.

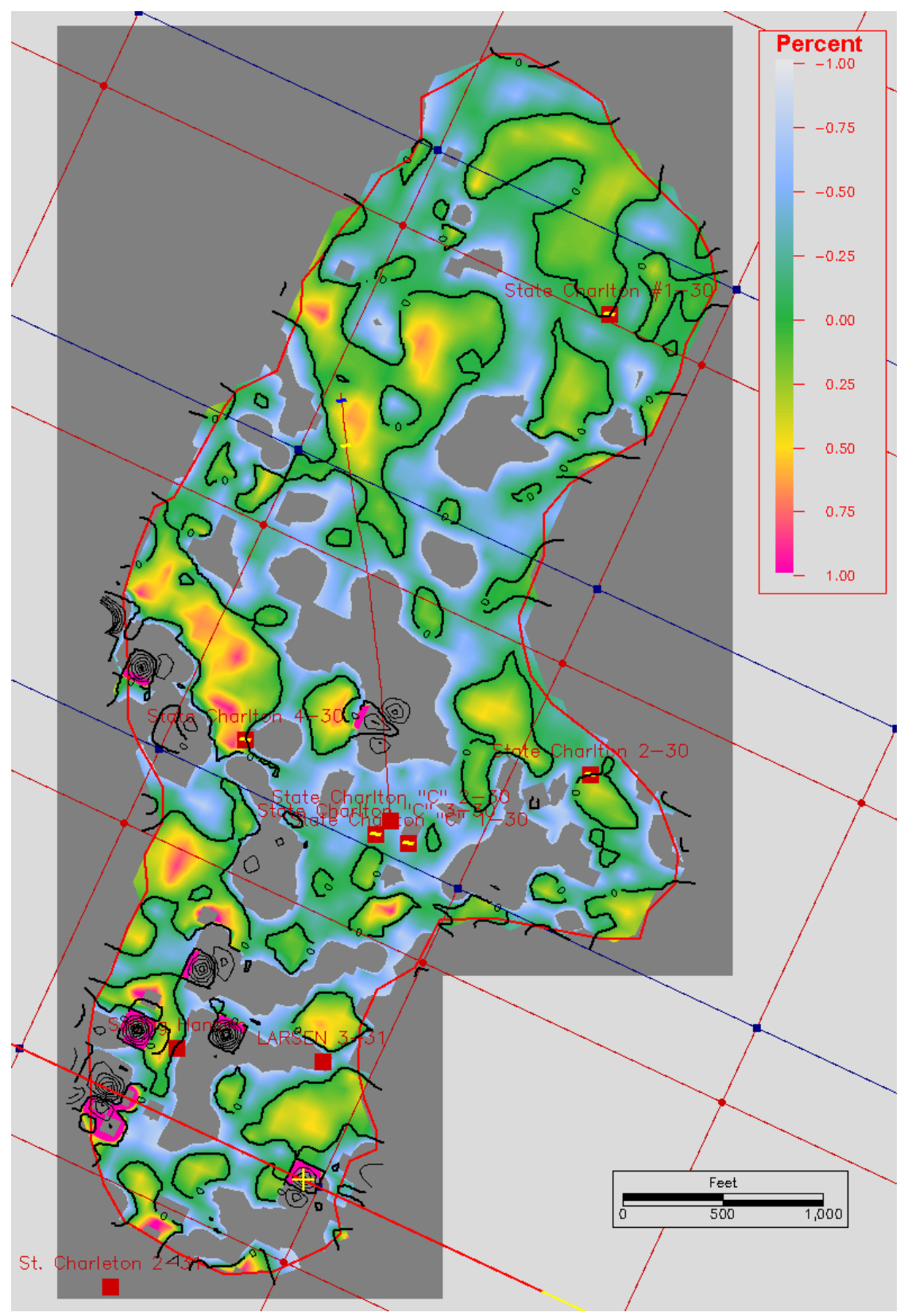

Figure 62: 4D amplitude difference for the A2-Carbonate. Differences are plotted as a percent amplitude difference map (Monitor-Baseline) with respect to $100 \%$ difference. Dark gray areas exceed $\mathbf{1 0 0 \%}$ difference. Colored areas have difference of less than $100 \%$. 
An amplitude difference map was then created by subtracting the non-reservoir A2Carbonate amplitudes (extracted from the peak events) from the adjusted Monitor survey from the Baseline survey (shown in Figures 61 and 62). This amplitude difference map of the A2-Carbonate was informative concerning the amplitude variation for this single stratigraphic unit and indicated that there were little changes between surveys' signal strength brought about due to differences in the acquisition geometry. Amplitude variations within the reef due to acquisition geometry or pore fluid replacement would not necessarily be confined to peak (+) events and could be associated with trough (-) events or some other (mixed) event.

It was decided that the best method to account for the signal strength variation due to acquisition geometry was the use of an amplitude percent difference approach for the non-reservoir A2-Carbonate. The amplitude difference grid for the A2-Carbonate events was then divided by the amplitude of the A2-Carbonate on the Monitor survey. This produced an amplitude percent difference grid between the adjusted A2-Carbonate amplitudes of the Monitor survey and the Baseline survey. This is shown in Figure 61.

The resulting percent difference grid contained several localities within the reef where the amplitude difference between the monitor and the Baseline surveys approached $7000 \%$. These were all in the southern half of the survey and are believed to be related to the issues previously discussed above that are due to the slight changes in survey geometry and the time of year when the surveys were acquired. These can easily be seen as bright red or blue points in the southern part of the survey shown on Figure 61. However, these are clearly isolated occurrences, restricted in all cases to single traces.

The vast majority of the northern portion of the reef showed an amplitude variation between the Baseline and the Monitor surveys of $100 \%$ or less. Figure 62 shows the A2-Carbonate amplitude difference in percent between the Monitor and Base surveys with the display scale set to $100 \%$ to $100 \%$. Those portions of the grid that are outside these ranges are displayed in dark gray. As can be 
seen in the northern portion of the reef, where reservoir simulations indicate the $\mathrm{CO}_{2}$ would be concentrated, there are small and isolated areas that have amplitude differences greater than $100 \%$, however, the majority of the grid is near the scale midpoint of $0 \%$.

Since the 4-D processing for both the Baseline and the Monitor surveys were identical, the difference in amplitude for the A2-Carbonate event (a non-reservoir stratigraphic unit with high acoustic impedance contrast with the overlying sedimentary unit) shown in Figure 61 and Figure 62, is believed to be due to minor differences in the acquisition of the two surveys. This is believed the result of the few, minor changes in the source and receiver geometry brought about due to licensing and landowner restrictions mentioned earlier.

The determination of the variation in signal strength due to acquisition provided the basis for the interpretation of amplitude differences noted between the Baseline and adjusted Monitor surveys. Throughout most of the northern portion of the reef amplitude differences of $100 \%$ or less could be attributable to acquisition related effects. Differences of greater than $100 \%$ would need to be examined in more detail as they could be attributable to changes within the reservoir or acquisition if they occur within those few areas denoted in dark gray on Figure 62.

In an attempt to compensate for slight variations in event time that may have occurred to the top of the reef it was decided that the 4-D interpretation would be conducted on seismic volumes that had been flattened on the A2 Carbonate. Since the A2-Carbonate is such a strong event and easily interpreted it could be used as a datum from which events in the reef could be referenced. This would minimize any time differences that could be significant when attempting to relate events within the two seismic surveys.

Time maps of both interpreted horizons (A2-Carbonate Baseline and A2-Carbonate Monitor) were generated and subtracted from one another. Flattened seismic volumes of the Baseline and Monitor 4-D seismic surveys were then generated using the A2-Carbonate as the flattened reference 
datum and moving this event to a time of $0 \mathrm{~ms}$. Comparisons were then made between these two volumes as well as the results of the reservoir simulation.

During this review a number of a few strong amplitude differences were noted. Figure 63 shows the crossline 5045 for both the Base (shown above in Figure 63) and Monitor surveys (shown below in Figure 63). These lines are displayed using the standard industry convention with the northeastern portion of the line on the right of the display. As can be seen on the percent amplitude difference map crossline 5045, shown as a blue line trending north northeast, is primarily located in the northern portion of the reef in areas with less than $100 \%$ amplitude difference. However, a significant amplitude difference was noted at the top of the Guelph reef between traces 68 to 75 . The highest amplitude difference that should be seen in this area is $50 \%$ (colored yellow).

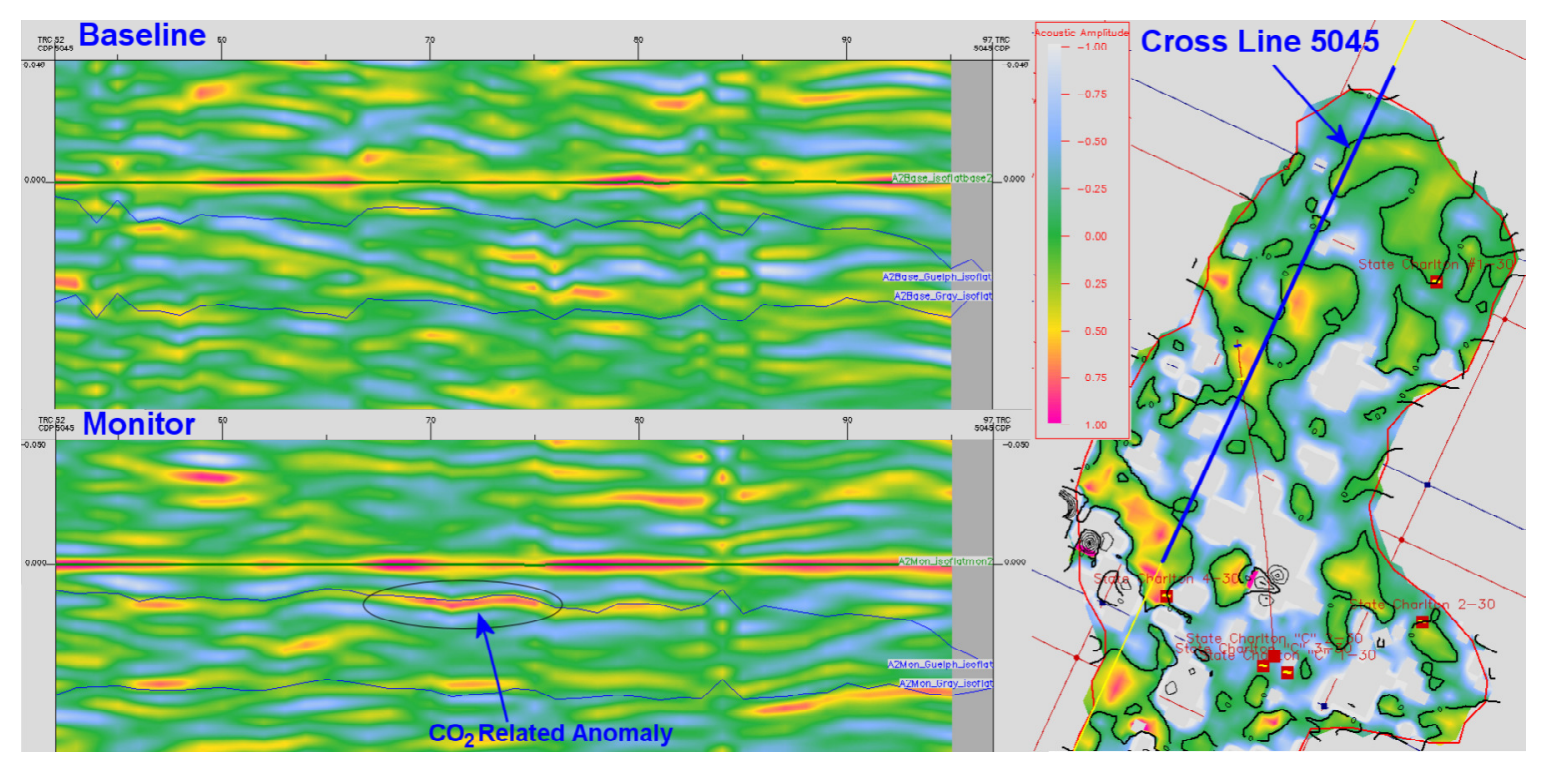

Figure 63: Crossline 5045 (Baseline survey above, Monitor survey below) flattened on the top of the A2 carbonate. Location of the crossline is shown as a blue line on the percent amplitude difference map for the flattened A2 carbonate event at right. Dark gray color indicates percentages beyond $100 \%$

Figure 64 shows the same two lines with the amplitude for a single sample on the same trace on both surveys being annotated. This sample, shown at the center of the black rectangle, has an amplitude value of -186 (shown above). As indicated for the area by the amplitude difference map for the A2 Carbonate (Figure 62) the maximum value for the area of 50\% the highest amplitude 
expected on the Monitor survey that would be related to differences in acquisition would be -279 or 93. The value for the same sample on the Monitor survey is 1420 . Not only is this a difference of over $700 \%$ but there is also a sign change. Therefore, this difference is too great to be associated with acquisition related differences.

It is also noted that this major increase in amplitude appears to be following the top of the reef as shown by the interpretation shown as a dark blue line. This interpretation was made during the initial stages of the project and was not updated. This observation is significant in that $\mathrm{CO}_{2}$ would be expected to rise within the reservoir due to its buoyancy and flow along the base of the reservoir's cap rock. The anomaly has this characteristic (Figure 64).

Figure 65 shows this same amplitude anomaly but along in line 1072. Again, on the maps shown on the right percentage values that exceed $100 \%$ are shown in dark gray. Again these lines are displayed using the standard industry convention with the eastern portion of the line on the right of the display. A short portion of this line does run across one of these areas east of the anomaly. However, an amplitude anomaly can be seen located on the western side of the reef in an area indicated to have almost $0 \%$ difference between the two surveys.

A sample from an area of significant difference that is believed to result from $\mathrm{CO} 2$ flooding of the area was selected for comparison and shows an amplitude of 1234 on the Baseline survey and 2101 on the Monitor survey, (see in Figure 66). If the maximum value in the area associated with amplitude variations associated with acquisition and processing is 50\% then the highest amplitude should be 1851 or less. The value of 2101 on the Monitor survey is more than $70 \%$ higher than the amplitude on the Baseline survey. This difference is not as great as the sampling described above along crossline 5045, however, the anomaly has the same orientation in that it occurs just below and following the upper surface of the reef. This orientation also supports the concept that this anomaly is associated with the injected $\mathrm{CO}_{2}$ plume. 


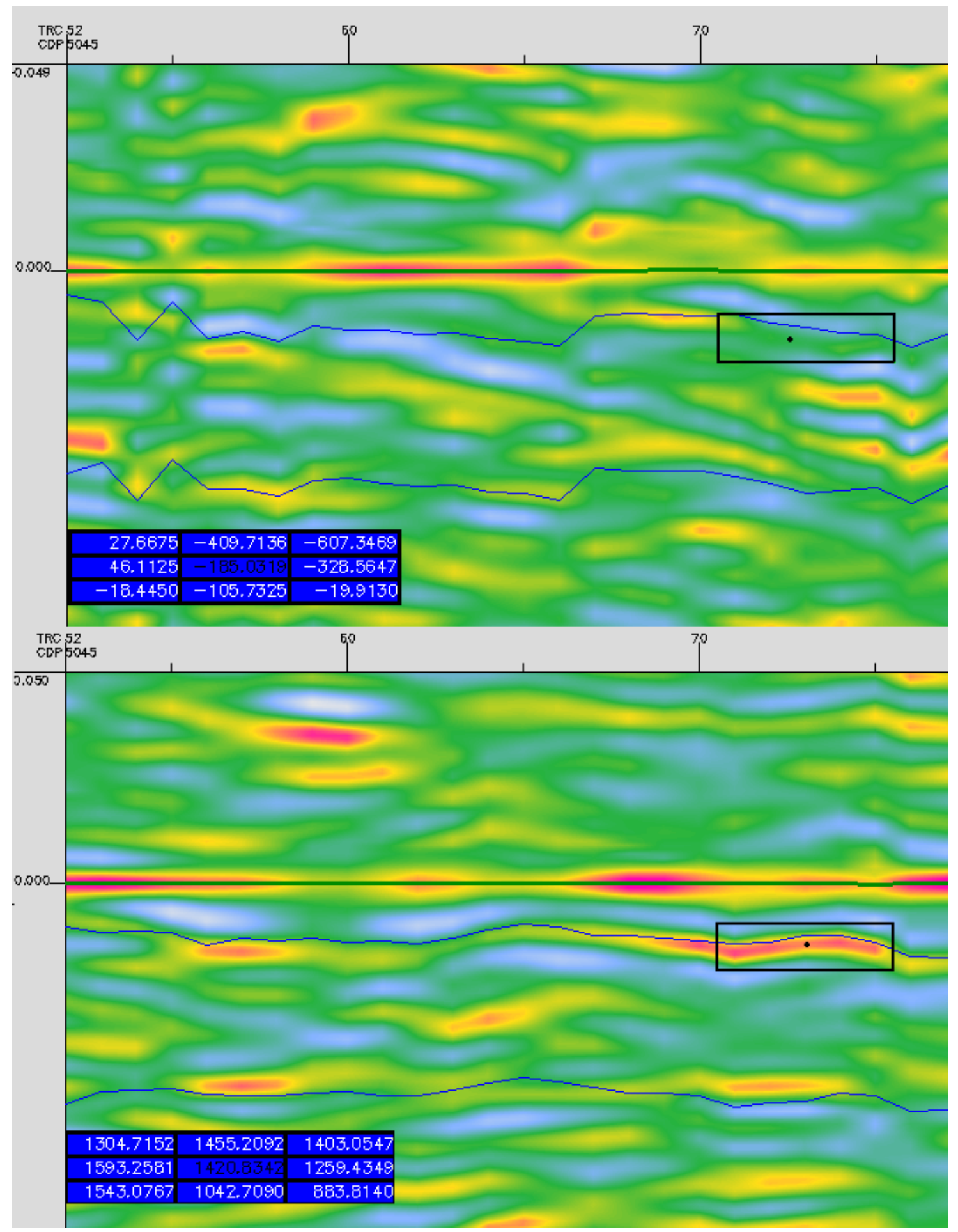

Figure 64: Crossline 5045 (Baseline survey above, Monitor survey below) flattened on the top of the A2-Carbonate with single sample amplitude annotated. 


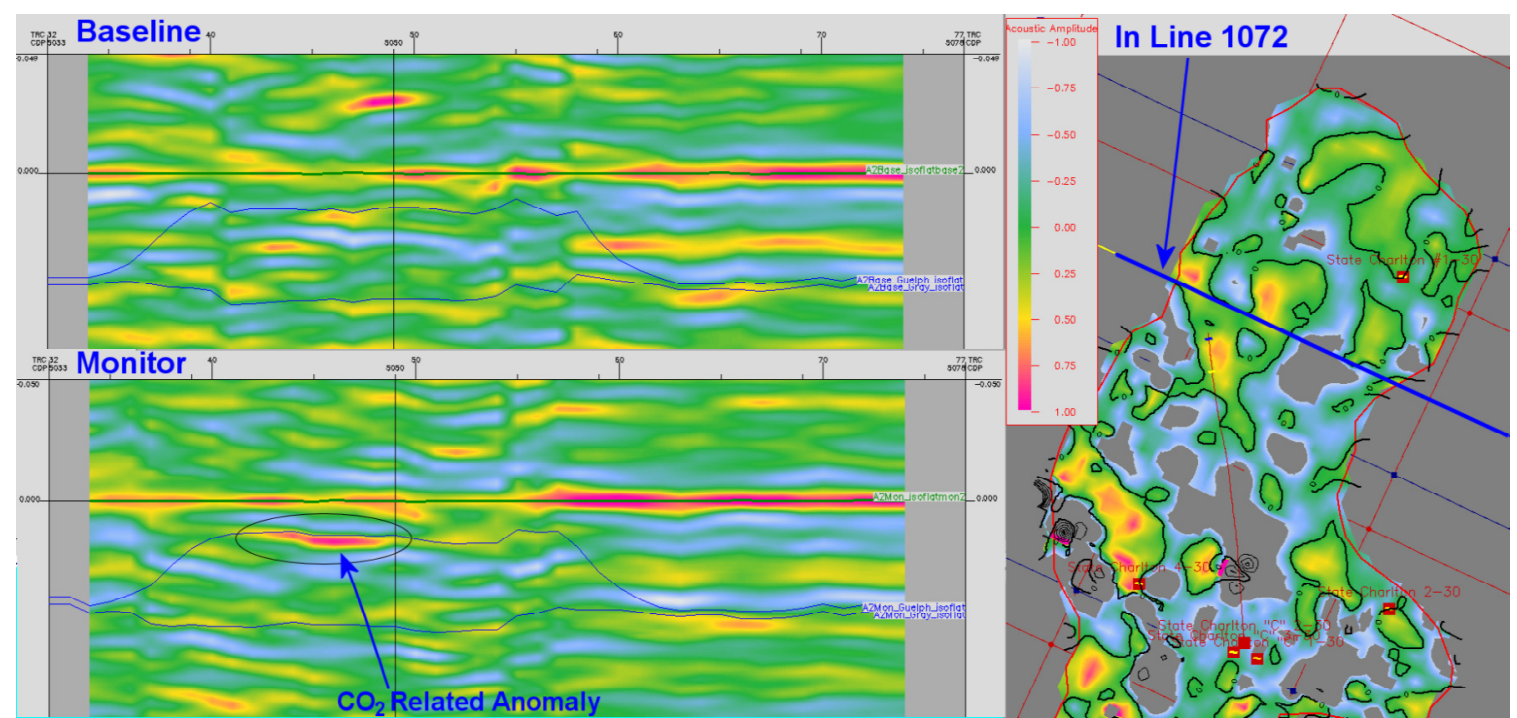

Figure 65: In-line 1072 (Baseline survey above, Monitor survey below) flattened on the top of the A2 carbonate. Location of the crossline is shown as a blue line on the percent amplitude difference map at right. Dark gray color indicates percentages beyond $100 \%$.

In addition to reviewing the flattened Baseline and flattened Monitor surveys using in-line and crossline comparisons a number of time slices through the flattened Monitor survey were generated and examined. In the flattened surveys, the upper surface of the Guelph formation in the northern portion of the reef occurs at approximately $10 \mathrm{~ms}$ in many places relative to the flattened A2 Carbonate event. Time slices through this Monitor seismic volume extending from 10 to $20 \mathrm{~ms}$ below the A2-Carbonate are presented in Appendix A. The 11 time slices through this $10 \mathrm{~ms}$ (90 foot range of depths) image approximately the upper quarter of the reef, as the reef base in this area occurs between 50 and $53 \mathrm{~ms}$ (450 feet) below the top of the A2 carbonate. The Guelph reef is approximately $40 \mathrm{~ms}$ (or 360 feet) two way travel time. 


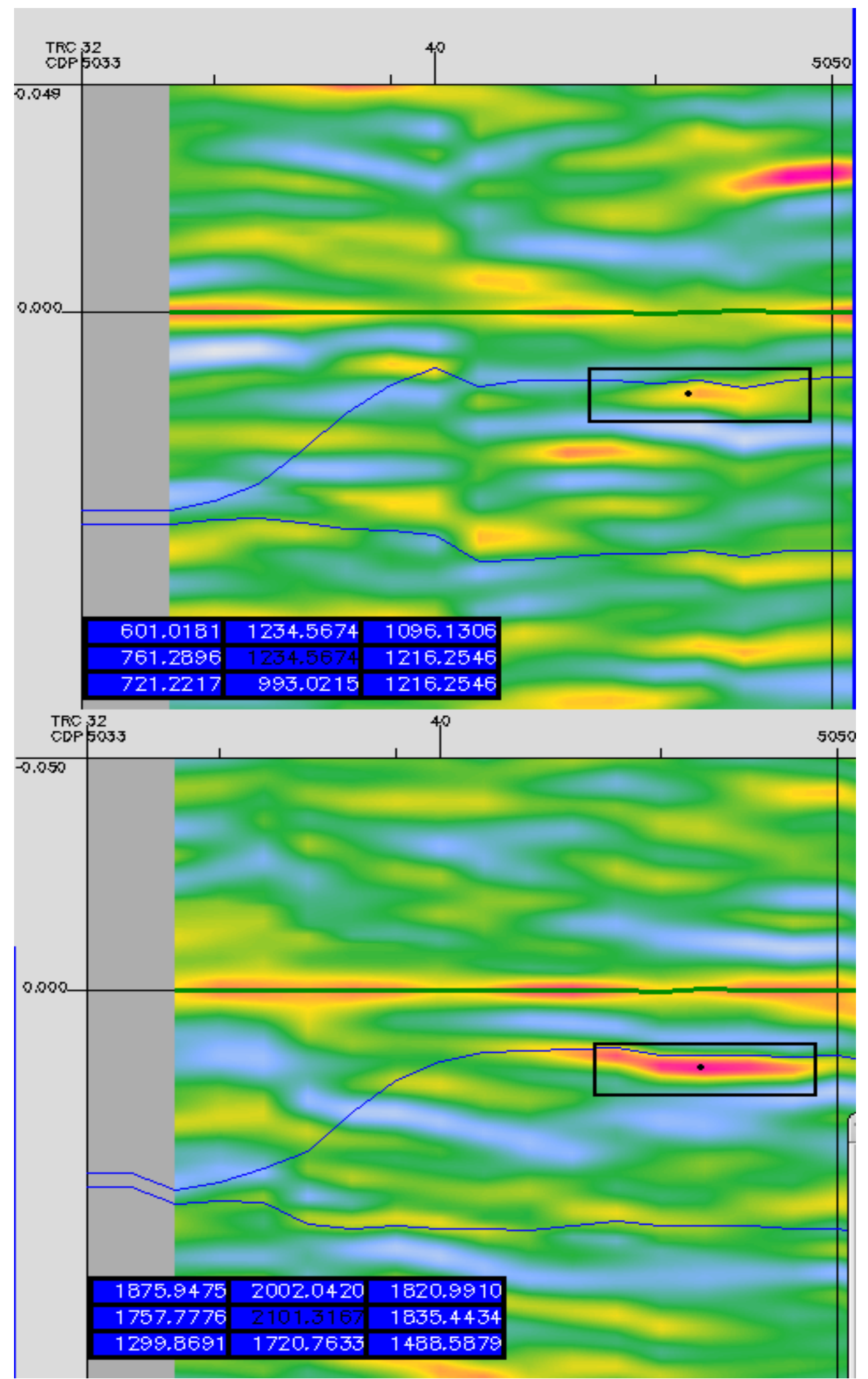

Figure 66: In line 1072, east to the right. (Baseline survey above, Monitor survey below) flattened on the top of the A2-Carbonate with single sample amplitude annotated. 
An examination of this sequence reveals a small high amplitude anomaly just east of the injection point, which occurs between the small yellow and blue markers along the Charlton "C"2-30 borehole (Figure 62). This anomaly grows in size and strength with subsequently deeper time slices. Time slices through the Monitor and Baseline seismic volumes extending from 10 to 20 ms below the flattened A2-Carbonate are presented in Appendices A and B.

Figure 67 shows the time slice located $15 \mathrm{~ms}$ below the top of the A2 carbonate on the flattened Monitor survey. The high amplitude anomaly at this depth has a slightly arcuate shape that extends from southwest to northeast from the injection point towards the enhanced oil recovery production well, the State Charlton \#1 - 30. The shape and location of this anomaly, which is referred to in the figure as the "jet", along with the amplitude evidence presented above, strongly suggests that this is the $\mathrm{CO}_{2}$ plume moving between the injection point and EOR well near the top of the reef. Other high amplitude anomalies can be seen occurring in this time slice just east of the jet and also just north of the EOR well.

The anomaly observed North and East of the EOR well is believed to be related to the geology of the reef itself and not related to fluid substitution since high amplitudes on both the Baseline and Monitor survey are observed in this area. This anomaly can be seen in Figure 68, which compares the Baseline and the Monitor surveys. This anomaly is the labeled 1. Additionally, the area indicated 2 is also likely due to the geology of the reef and not fluid substitution. However, the anomaly labeled 3 clearly shows a significant increase in amplitude, which has been shown in the preceding figures to be significantly greater than amplitude changes associated with acquisition differences. Amplitude anomaly 3, also referred to as the high amplitude "jet" is highly significant with regard to the results obtained during the Department of Energy portion of this study.

As mentioned in the previous section "Reservoir Simulation" the reef's porosity distribution developed during the static modeling and used by the subsequent reservoir simulation and which was based on the instantaneous frequency volume derived from the full azimuthal baseline survey, was 
judged to be good by the production history matching process performed during the reservoir simulation. However, as noted the area between the State Charlton "C" 2-30 borehole (the $\mathrm{CO}_{2}$ injector) and the State Charlton \#1-30 borehole (the EOR producer) display localized interference effects that required additional adjustment of porosity distribution through the static model. This localized interference detected during the reservoir simulation is believed related to the presence of fractures, not characterized during this portion of the study which result in this high amplitude "jet" and will be discussed at greater length in the conclusions portion of this chapter. These fractures may be related to dissolution features in the reef created during subaerial exposure (Sears and Lucia, 1980).

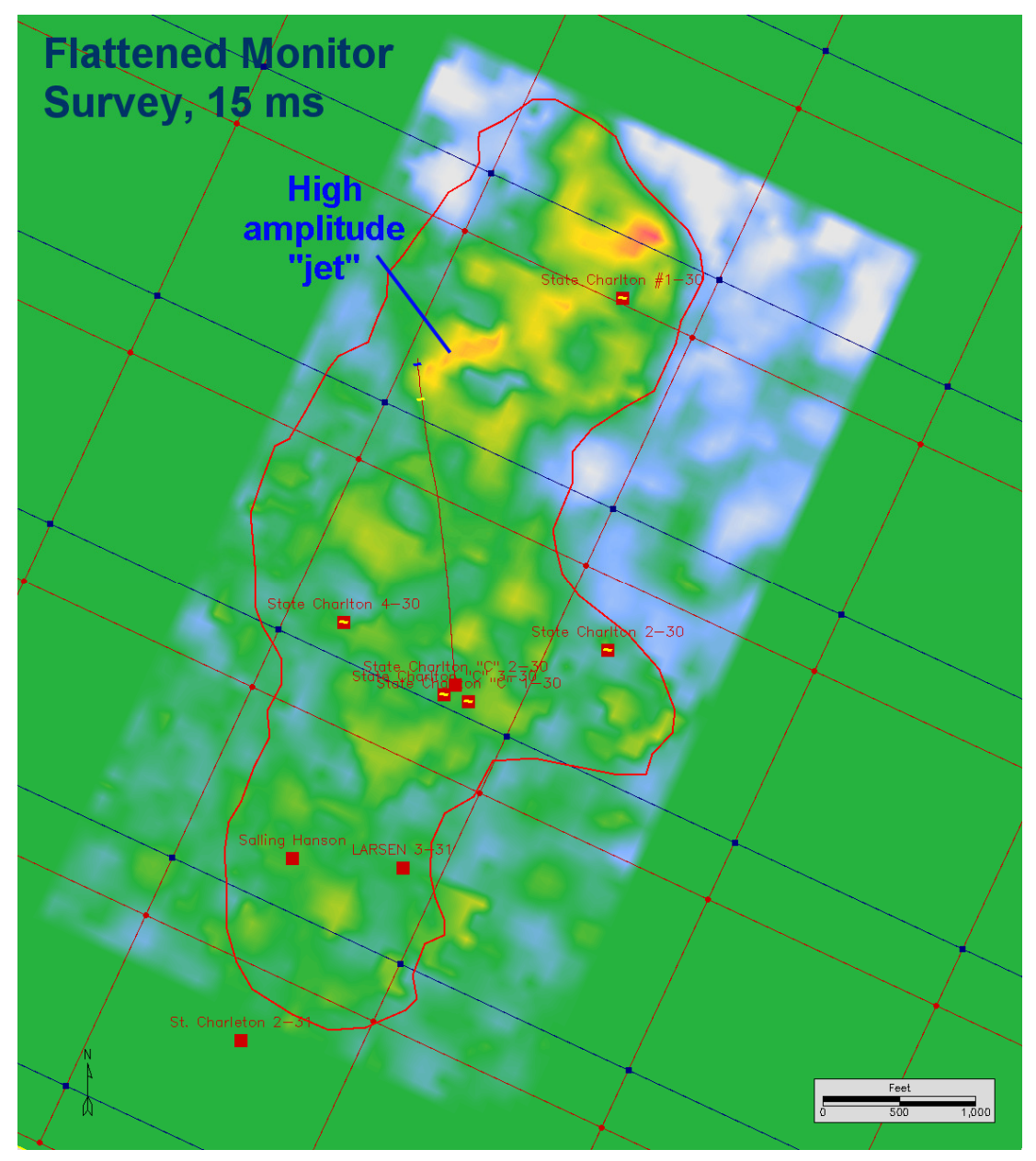

Figure 67: Time slice $15 \mathrm{~ms}$ from the A2-Carbonate flattened Monitor survey showing a high amplitude anomaly" just east of the injection point "jetting" toward the enhanced oil recovery production well. 


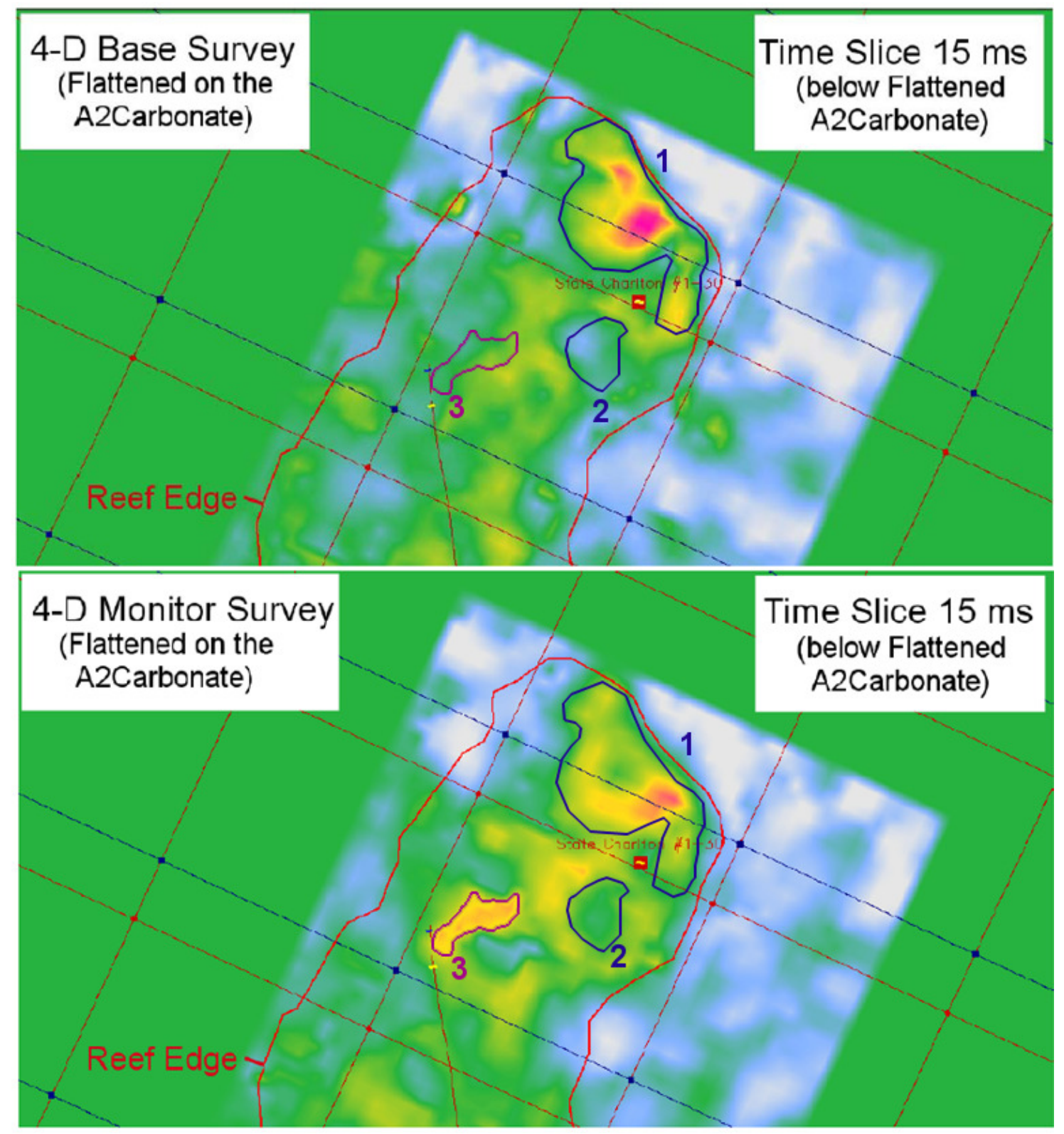

Figure 68: Comparison between time slice $15 \mathrm{~ms}$ from the A2 carbonate on both Baseline and Monitor surveys.

Figure 69 shows the time slice $17 \mathrm{~ms}$ below the top of the A2-Carbonate. Amplitude anomaly 3 has altered at this depth, indicating a strong narrowing of the amplitude near the injection point. At this point the anomaly appears to bifurcate into two arms that extend into the area immediately west of the EOR well (designated 2 in Figure 68). The southern arm is slightly decreased in amplitude while 
the northern arm appears to be taking a slightly more contorted path between the injection point and the EOR well. This suggests that the northern anomaly contains more $\mathrm{CO} 2$ and has a more contorted flow path. The feature in the image is interpreted to be associated with a larger concentration or pool of $\mathrm{CO}_{2}$ just northwest of the State Charlton \#1-30 borehole.
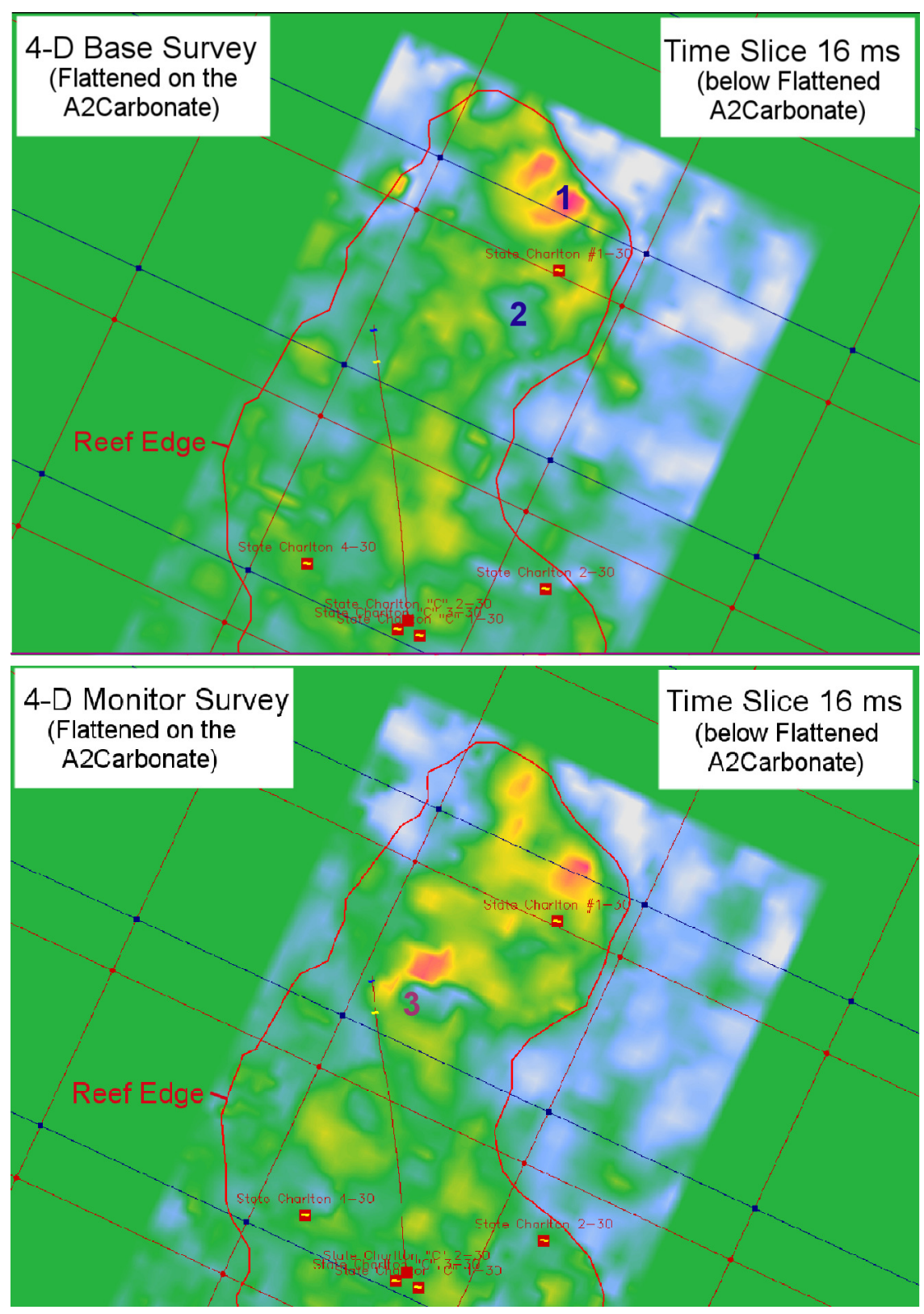

Figure 69: Comparison between time slice $17 \mathrm{~ms}$ from the A2 carbonate on both Baseline (above) and Monitor (below) surveys. 
Figure 70 shows time slice $20 \mathrm{~ms}$ below the top of the A2-Carbonate. At this depth the amplitude of the anomaly decreases but still occupies the area immediately to the west of the EOR well.

The amplitude anomalies and their relation to the injection and producing wells provide strong support for the interpretation that they are produced by $\mathrm{CO}_{2}$ flooding in this area. The location and shape of these anomalies are consistent with our expectations that $\mathrm{CO}_{2}$ should move from the injector point to the producer well along high permeability and porosity pathways within the reservoir. The $\mathrm{CO}_{2}$, injected in the State Charlton "C"2 - 30 borehole approximately 3/4 of the way into the reef, migrates upward until encountering the seal at the top of the reservoir. From this point it moves northeast as suggested by the anomaly shape observed on time slice $15 \mathrm{~ms}$. Approximately half way between the two wells (in map orientation view) $\mathrm{CO}_{2}$ migration is interpreted to encounter a zone of low porosity/permeability (low flow) and is forced around that area. These amplitude anomalies rejoin just to the northeast of the EOR well.

This evidence provides strong support for the potential use of time-lapse surface seismic monitoring to detect injected $\mathrm{CO}_{2}$ migration paths through complex reservoirs, even at depths of over 5000 feet and in high velocity carbonate rocks

The results of the reservoir simulation, which were ultimately based on the static model generated with the porosity distribution obtained through the instantaneous frequency analysis, were compared to the A2-Carbonate flattened Monitor seismic volume. The purpose of this comparison was to determine if the high amplitude anomalies, that are interpreted to indicate the presence of injected $\mathrm{CO}_{2}$, correspond to the high porosity zones in the model validated by history matching reservoir simulations. 


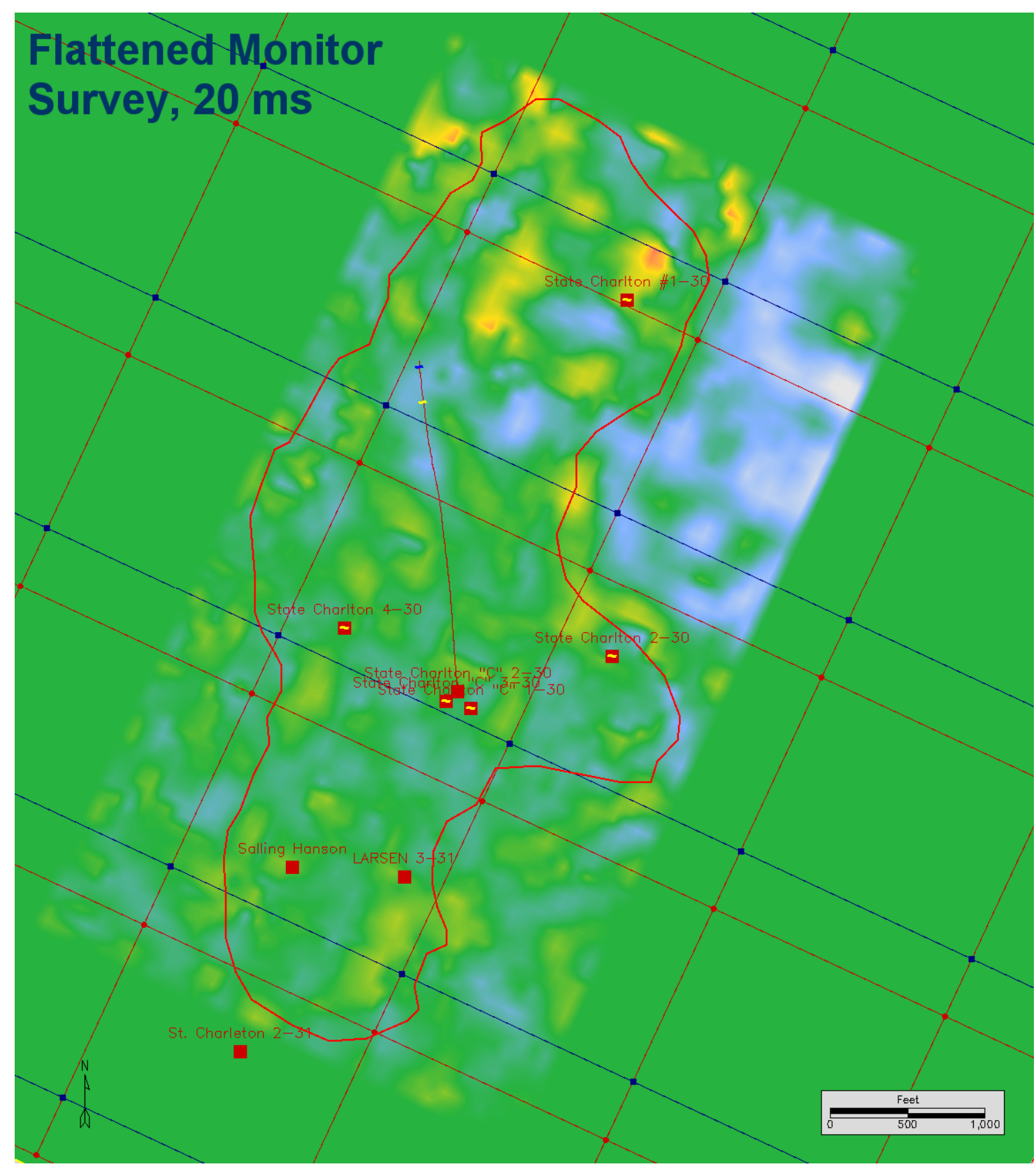

Figure 70: Time slice $20 \mathrm{~ms}$ from the A2-Carbonate flattened Monitor survey showing that the high amplitude anomaly appears to be weakening in strength but still suggesting a horseshoe shaped orientation around the area immediately west of the State Charlton \#1 - 30 well. 
Figures 71 and 72 show this comparison for the same time slices discussed above and their corresponding reservoir simulation layers. Figure 71 shows time slice $15 \mathrm{~ms}$ and the corresponding reservoir simulation layer, \#9. Note that in the reservoir simulation displays only $\mathrm{CO}_{2}$ concentrations of $60 \%$ or higher are shown. High amplitudes are shown east of the injection point, however, the interpreted $\mathrm{CO}_{2}$ anomaly is not obvious within the simulation layer. The reason for this at the conclusion of the Department of Energy study was unknown, however, it was interpreted that the anomaly was produced by $\mathrm{CO}_{2}$ movement along a linear trending, open natural fracture system. The long, linear orientation of this amplitude anomaly supports this theory.

Figure 72 shows time slice $16 \mathrm{~ms}$ and its corresponding reservoir simulation layer \#10. Note that the amplitude anomaly increases east of the injection point.

The locations of the amplitude anomalies noted on the flattened, scale adjusted Monitor survey and the reservoir simulation modeled $\mathrm{CO}_{2}$ porosity distributions cannot be considered coincident. However, given the large number of variables involved in the seismic acquisition, processing, attribute interpretation, porosity modeling and reservoir simulation, the relationship suggested must be considered more than tenuous. 

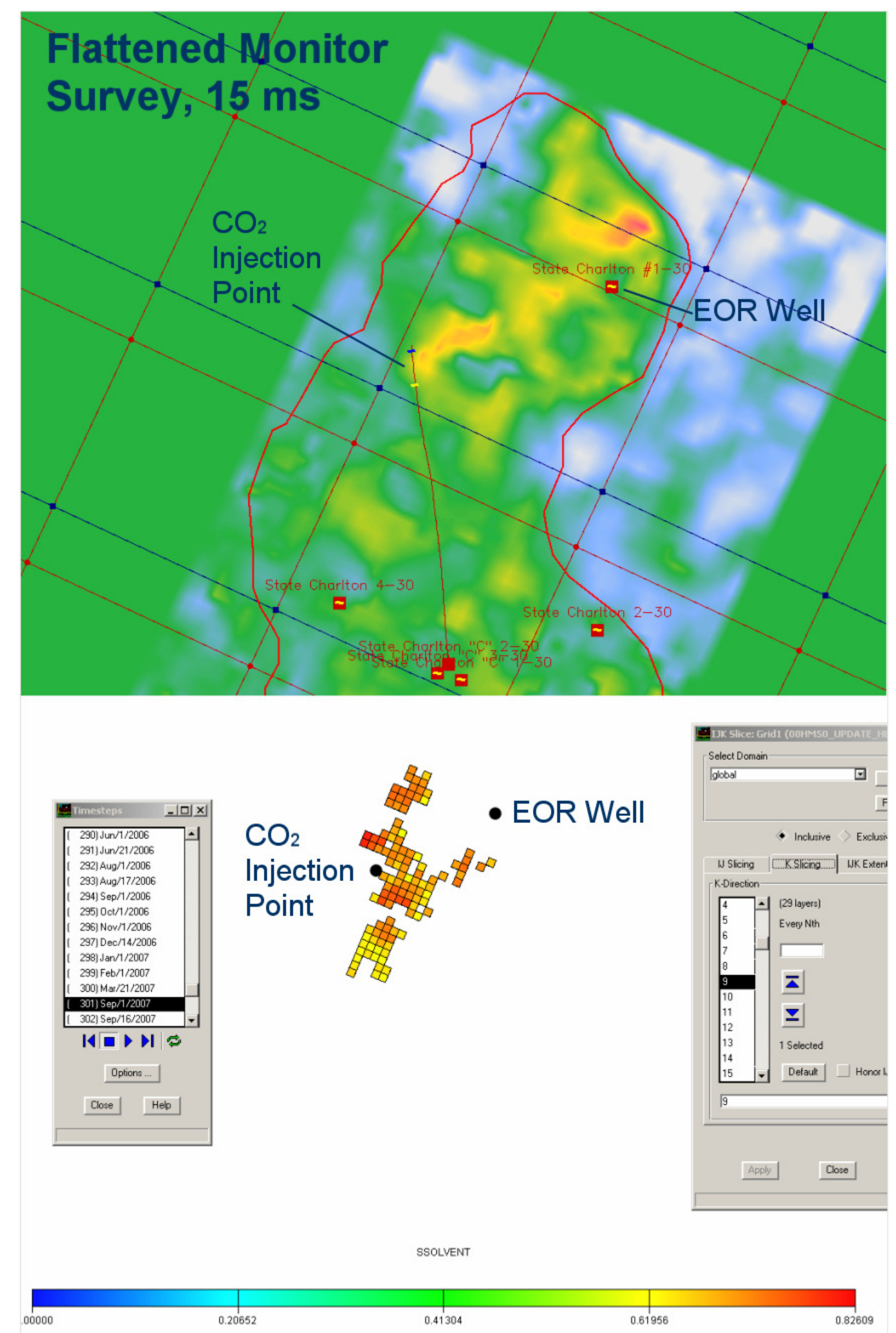

Figure 71: Top - time slice $15 \mathrm{~ms}$ below the flattened A2-Carbonate. Bottom - Layer 9 from the final, predictive reservoir simulation (time step September 2007) which corresponds to the approximate depth of the time slice. 

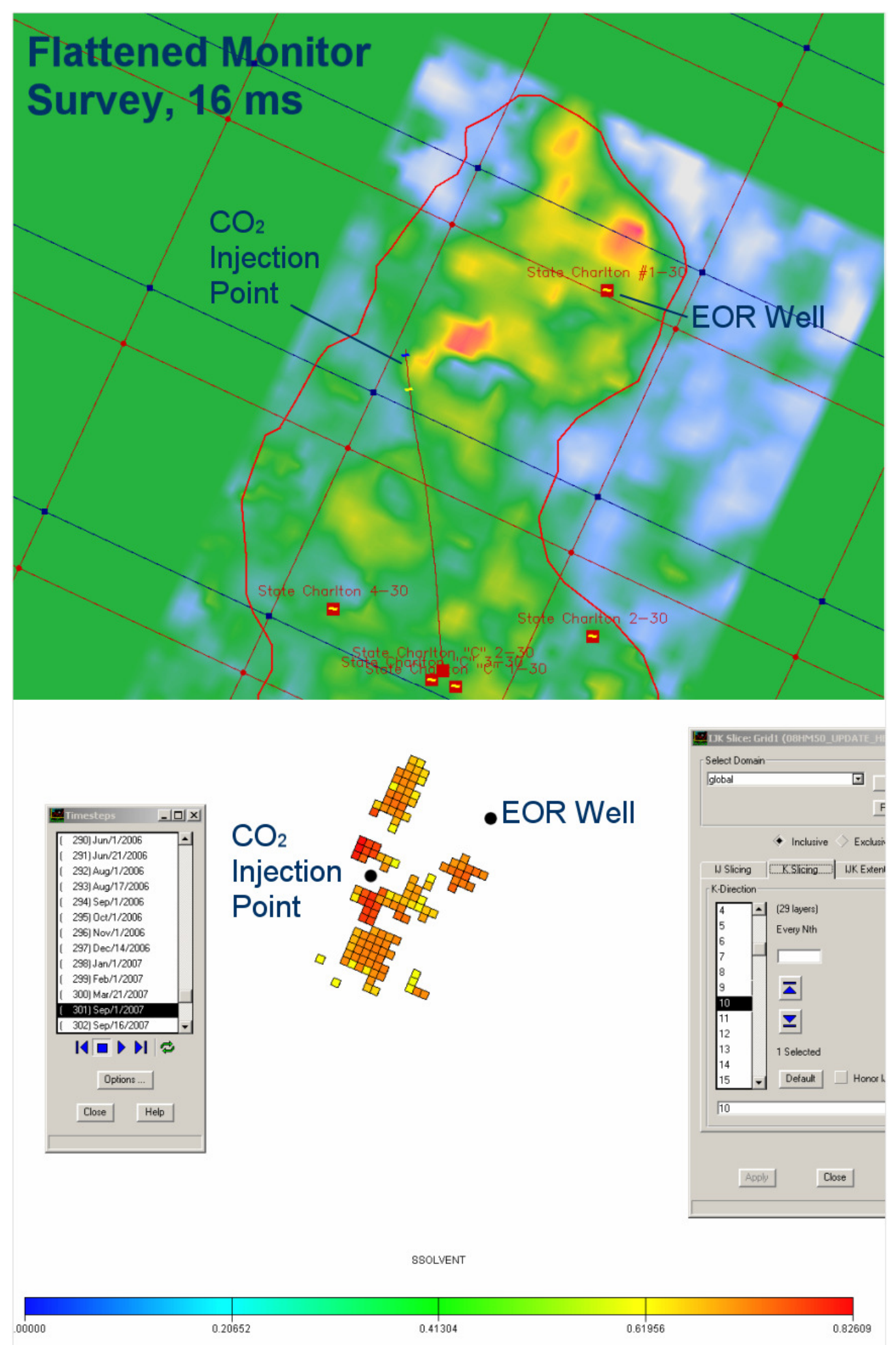

Figure 72: Top - time slice $16 \mathrm{~ms}$ below the flattened A2-Carbonate. Bottom - Layer 10 from the final, predictive reservoir simulation (time step September 2007) which corresponds to the approximate depth of the time slice. 


\subsection{Conclusions}

The Department of Energy portion of this research demonstrated that state-of-the-art, costeffective, geophysically-based reservoir characterization technologies can be used to effectively image moderately deep, carbonate reservoirs. These technologies, when combined in a fully integrated geoscience/reservoir engineering approach, have been shown to produce a good understanding of key reservoir properties within these types of fields. This integration has included the following technologies;

- Rock property determination

- Forward seismic acquisition modeling

- 3-D seismic acquisition

- Seismic wavelet determination and analysis

- Well to seismic time generation

- Seismic frequency analysis

- Seismic attribute generation and interpretation

- Reservoir engineering data analysis

- Reservoir characterization methodologies

- Reservoir simulation

- $\quad$ Production history matching

These technologies have been combined to the extent that reservoir controlling properties, such as the drained, high flow systems, have been accurately mapped out and used in the planning of $\mathrm{CO}_{2}$ based enhanced oil recovery projects. The porosity predictions made prior to the drilling of the two new wells in the Charlton 30/31 Field were based on the porosity/instantaneous frequency relationship. High porosity intervals encountered by the well bore provide strong support for the porosity/instantaneous frequency relationship. 
This relationship is also supported by the association between the high amplitude anomalies observed at the top of the reservoir within the flattened A2-carbonate Monitor volume and the reservoir simulation predictions for the higher concentrations of $\mathrm{CO}_{2}$ within the reef. To a large extent the amplitude anomalies correspond to areas predicted to be $\mathrm{CO}_{2}$ filled. Additionally, the porosity volume developed using the instantaneous frequency attribute from the Baseline 3-D survey is supported by the reservoir simulation history match.
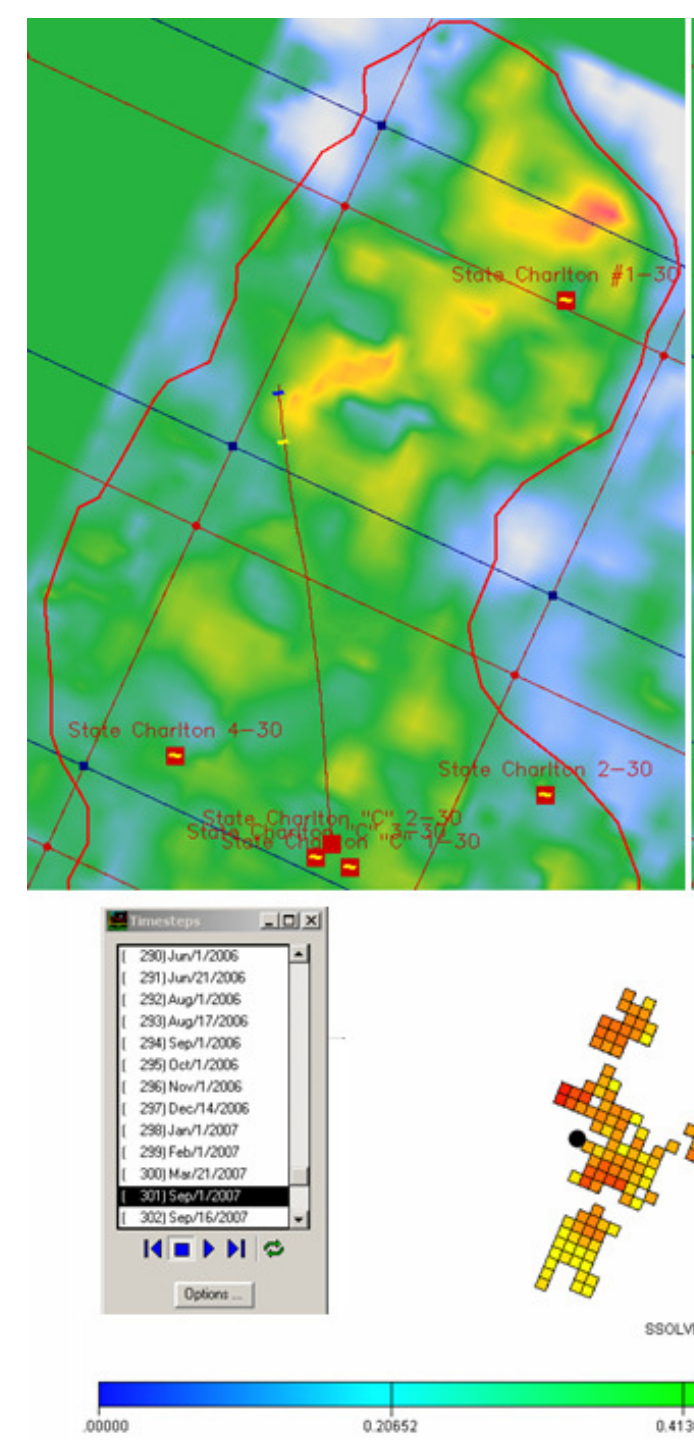

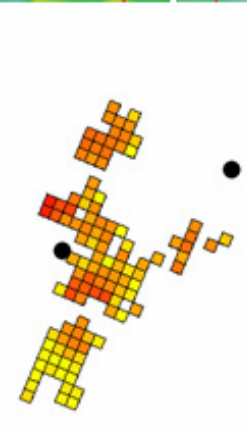

SSOLVENT
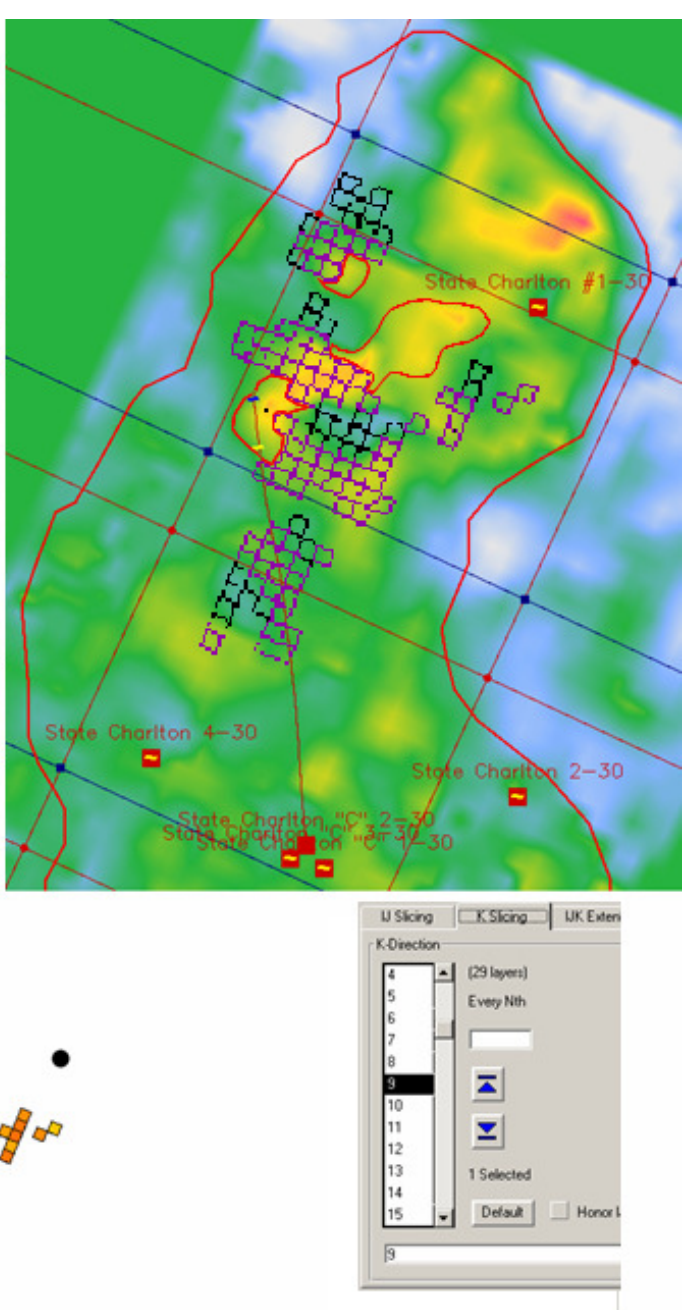

0.82609

Figure 73: Same display shown in Figure 71 except image added into the upper right to show the time slice overlain with grid cells from the predicted reservoir simulation. See text for further explanation. 
Figure 73 shows a comparison of the high amplitude anomalies observed on the Monitor survey interpreted to be caused by the injected $\mathrm{CO}_{2}$ and those areas predicted by the forward looking reservoir simulation to be filled with $\mathrm{CO}_{2}$. Both correspond to the same time period, September 2007 when the Monitor survey was acquired. Reservoir simulation grid cells predicted to contain $\mathrm{CO}_{2}$ and which show correspondence with increased amplitude on the Monitor survey are outlined in purple. Those grid cells predicted to contain $\mathrm{CO}_{2}$ but which do not correspond with increased amplitudes are outlined in black. As can be seen from this illustration the vast majority of the areas predicted by the reservoir simulation to contain $\mathrm{CO}_{2}$ at this time also have increased amplitude. This visual correlation suggests that the injection of $\mathrm{CO}_{2}$ into carbonate reservoir can be accurately modeled and also validates the method used to detect and map the high porosity zones within the reef.

Figure 73 reveals the presence of areas within the reservoir believed to be filled with $\mathrm{CO}_{2}$ that were not predicted by forward-looking reservoir simulation. Two of the interpreted $\mathrm{CO}_{2}$ regions are associated with a high amplitude anomaly observed in the monitor survey. This area also corresponds to an area identified during the reservoir engineering analysis as an area of interference between the $\mathrm{CO}_{2}$ injector and the EOR well, see section 4.3 of this chapter.

The accurate mapping of reservoir properties combined with detailed reservoir simulations can provide optimized and detailed plans for conducting enhanced oil recovery projects in the future. These optimized EOR projects will be the key to recovering not only the potential 168 million barrels of oil contained in the Silurian reef trends of the Northern and Southern Michigan basin but also the billions of barrels of oil still contained in other reservoirs of the same type throughout the world.

Additionally, these technologies may be applicable to the characterization of reservoirs under consideration for $\mathrm{CO}_{2}$ sequestration projects. The identification of high porosity zones within carbonates would help optimize wellbore placement and $\mathrm{CO}_{2}$ injectability.

This portion of the study has also demonstrated that the monitoring of critical-phase $\mathrm{CO}_{2}$ injected into moderate depth, carbonate reservoirs can be accomplished using state-of-the-art, cost- 
effective, 4D seismic technologies. While this project attempted precise 3-D survey repeatability it was not entirely achieved.

Strong seismic amplitude anomalies were observed in the Monitor survey. These amplitude anomalies were interpreted to correspond approximately to zones of higher $\mathrm{CO}_{2}$ concentrations predicted by the reservoir simulation in a number of locations. The amplitude differences observed between the Baseline and Monitor surveys are much larger than amplitude anomalies interpreted to result from differences in acquisition and processing. Amplitude anomalies interpreted to result from $\mathrm{CO}_{2}$ flooding are not only located within the upper portion of the reservoir but appear to follow its upper surface, as predicted by reservoir simulation. This illustrates that the Monitor survey identified aerial and vertical extents $\mathrm{CO}_{2}$ flood within the reservoir.

In general, comparison of the results obtained from the Baseline and Monitor surveys support the proposed relationship between instantaneous frequency and porosity. The results obtained in this case history demonstrate their potential use for the planning of additional enhanced oil recovery wells, and recovery optimization from these reservoirs. Recommendations for the drilling of additional wells into zones of higher porosity containing additional oil reserves were made as a result of this study.

The use of 4D seismic to monitor $\mathrm{CO}_{2}$ based EOR projects will not only allow the adjustment of injector locations and injection parameters but will also help to identify zones that have been bypassed by the $\mathrm{CO}_{2}$ sweep. These bypassed zones can then be targeted with additional boreholes. As a result a significant amount of the 168 million barrels of oil in the Northern and Southern Michigan basin reef trends could be recoverable.

The application of this technology to image critical phase $\mathrm{CO}_{2}$ injected into carbonate reservoirs has implications outside of the enhanced oil recovery industry. The results presented in this chapter illustrate that 4D surface seismic technology provides useful information for the planning and implementation of large scale $\mathrm{CO}_{2}$ storage efforts. Surface, P-wave 3-D seismic surveys can be 
acquired over large areas prior to the drilling of wells. Interpretation of these surveys will improve reservoir characterization and evaluation of cap rock integrity. Once injection has commenced and progressed to a certain point additional 3-D surveys can be acquired to extend the time lapse coverage. Extended time lapse views will help ensure that injected $\mathrm{CO}_{2}$ remains within the targeted reservoir and that cap rock integrity is maintained. 


\section{CHAPTER 5 - THEORY AND METHODOLOGY}

\subsection{Theory and Conceptual Model}

High porosity "zones", and their corresponding high permeability, within the reef may occur as either concentrations of higher, and possibly vuggy, matrix porosity, or as open, natural fracture trends (see Figure 74). These two types of drained, high flow systems have distinctively different morphologies. Zones of higher, vuggy porosity will occur as irregular shapes that are scattered throughout the reef. These will transition from low porosity limestone to higher porosity dolomite with the high-end porosity values varying widely from zone to zone.

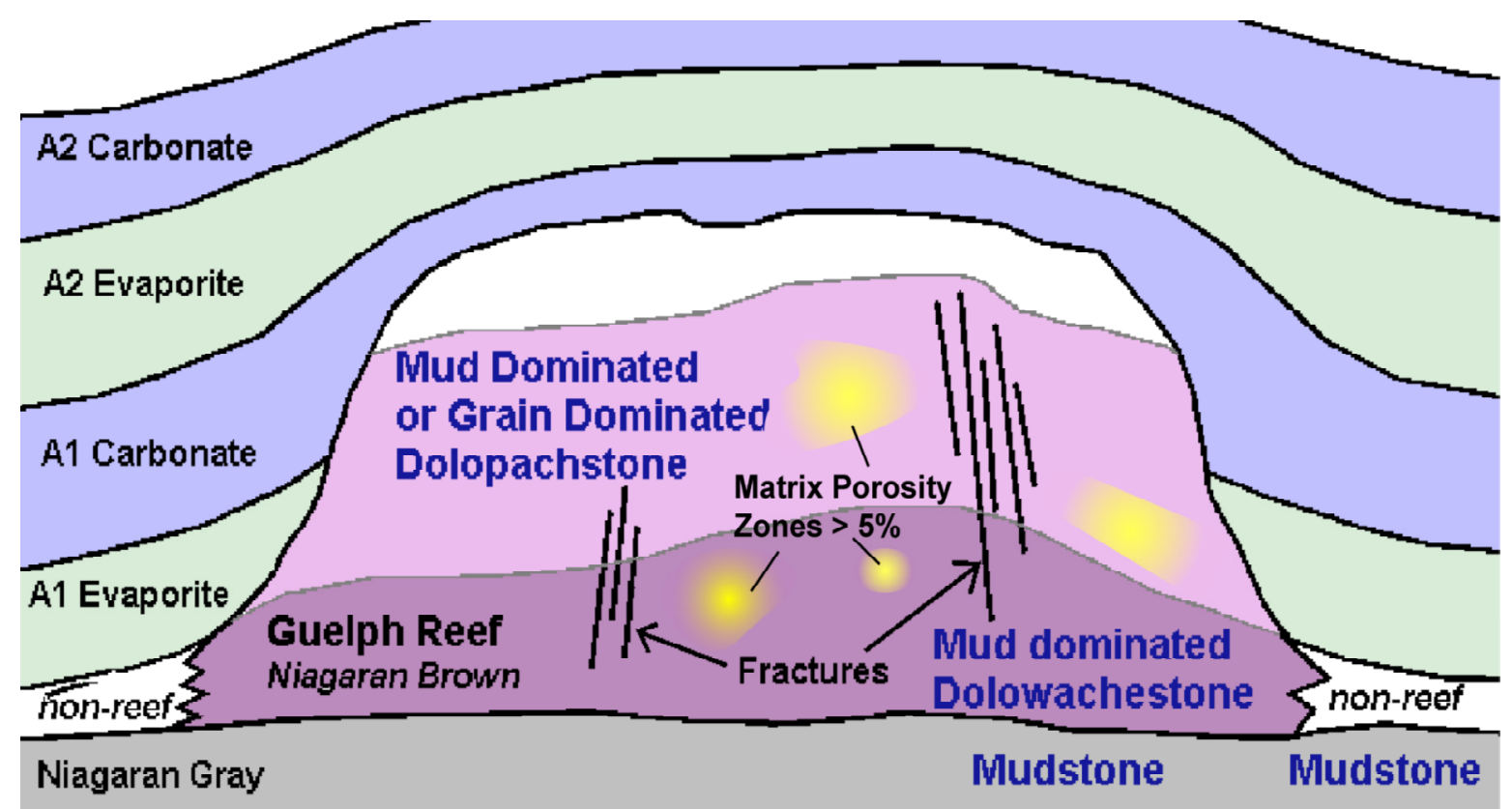

Figure 74: Illustration of matrix and fracture porosity / permeability zones within the reef.

Natural fractures generally have planar geometry. They often occur in zones or swarms. Fracture networks generally consist of more than one fracture set (trend). Open mode fractures are parallel to the maximum principal compressive stress direction and open in the least compressive stress direction. Mineralization can hold mode 1 fractures open even if the maximum compressive principal stress direction changes through time. Orthogonal sets of open mode fractures can develop coevally in response to local stresses produced by buckling or differential compaction. A single 
fracture set will produce acoustic anisotropy in directions parallel and perpendicular to the mean fracture plane orientation. A hypothesis of this research is that the influence of a single fracture set can be identified using spectral decomposition of azimuthal seismic volumes. The presence of multiple fracture sets will complicate the spectral response in individual azimuthal volumes. However, anisotropy revealed in more complexly fractured media may reveal trend orientations or azimuthal variation in the average properties (velocity and frequency response, for example).

\subsection{The Nature of Azimuthal Seismic}

Typically, 3-D seismic data, once acquired, are processed using all available shot/receiver pairs. This results in a single CDP trace that incorporates all source-receiver azimuths. Separate "azimuthal" seismic volumes are developed by selecting shot and receiver pairs that fall within a selected azimuth range (angular swath) with relation to the CDP. Figure 75 illustrates this relationship for a single CDP.
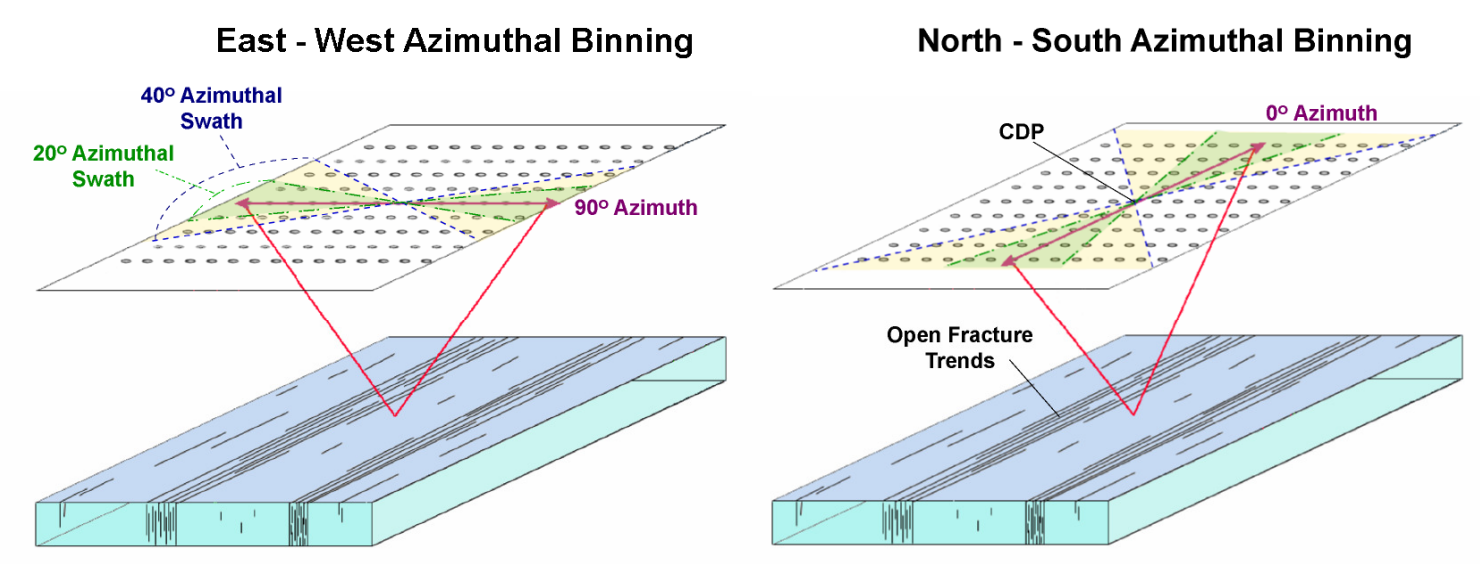

Figure 75: Azimuthal volumes are developed by binning all of the source / receivers within a specific angular swath.

As discussed in the Department of Energy Project Description section of Chapter 3 the geometry of the drained, high flow systems within the reservoir should affect the seismic response. 
Large zones of high matrix porosity will have isotropic azimuthal response for all seismic attributes. However, for zones of open natural fractures, which are typically linear in nature due to the stress state in the subsurface, an anisotropic response to various seismic attributes should be expected. The selection of the azimuth about which the angular swath will be centered for the development of the azimuthal seismic data is an important factor in the detection of open, natural fracture trends. If the dominant open fracture trend in an area is known at the time the 3-D seismic data are processed, azimuthal volumes parallel to and perpendicular to dominant, open fracture trend can be developed. These volumes represent primary azimuthal volumes upon which fracture detection methods could be employed and tested. If these fracture trends are unknown, then azimuthal volumes could initially be developed and inspected in the crossline and in-line directions.

Additionally, it is desirable to obtain azimuthal volumes at $45^{\circ}$ to these primary azimuthal volumes. These secondary azimuthal volumes can be used to refine the fracture trend interpretations, as the effect of the fractures on the seismic may be more or less pronounced in the secondary volumes. This also allows for a change in the fracture trend to be detected or the detection of fracture trends that may not have been anticipated or observed in the boreholes.

The amount of angular swath used in the development of these azimuthal volumes is also a major consideration. The angular range should be selected in order to maximize fold within individual azimuthal volumes; however, it is also desirable to maintain similar fold within the various azimuthal volumes. Maintaining similar fold may require narrowing or widening of the angular swath in order to obtain sufficient angular fold within all azimuthal volumes. Angular swaths greater than $45^{\circ}$ should not be used, since the overlap between the azimuthal swaths would result in some blurring or mixing of the differences associated with individual azimuths.

This study employed $45^{\circ}$ azimuthal steps with fan-angles equal to 45 degrees. These variable azimuth volumes are also referred to as azimuth-limited seismic volumes. The variable azimuth observations can then be interpreted to provide information concerning azimuthal anisotropy in the 
seismic properties associated with the reservoir and bounding intervals. Variable azimuth 3D seismic volumes are used in this study to detect and determine the orientation and geometries associated with drained, high flow systems in order to characterize the reservoir.

\subsection{Spectral Decomposition Description}

Castagna (2006) defined spectral decomposition as "any method that produces a continuous time-frequency analysis of a seismic trace". In his paper he described a number of methods that can be used for this type of analysis. These include the DFT (discrete Fourier Transform), MEM (maximum entropy method), CWT (continuous wavelet transform), and MPD (matching pursuit decomposition) which he discussed in detail.

The method employed to produce the iso-frequency volumes used in this study was Schlumberger's Cosine Correlation Transform (CCT). This technique can be employed in two forms; Frequency Indexed and Iso-Frequency. The Iso-Frequency methodology produces seismic amplitude volumes for specific frequencies from the original full-frequency volumes. The iso-frequency method has been employed for this study.

\subsection{Description of Interpretation Methodology}

The portion of the study that was conducted for the Department of Energy revealed the presence of a significantly negative correlation between increases in porosity (above 5\%) and instantaneous frequency within the reservoir. This relationship along with various supporting evidence was discussed extensively in Chapter 4 . The analysis presented in Chapter 4 focused on the frequency analysis of a full azimuthal seismic volume. That analysis was undertaken to identify low frequency zones interpreted as zones of drained, high flow systems within the reservoir causing frequency attenuation within the Charlton 30/31 Oil Field.

I have theorized that a similar approach could be used to differentiate between drained, high flow systems within the matrix and narrow linear drained, high flow systems associated with open 
fracture trends. This can be accomplished through the analysis of variable azimuth (or azimuthlimited) seismic volumes. The analysis of variable azimuth seismic volumes will be used to locate zones of low frequency that are a function of variable azimuth. This analytical approach will allow differentiation between zones that have isotropic or anisotropic (aligned) distribution. Figure 16 and Figure 17 illustrate how this would be accomplished. Isotropic, drained, high flow systems (regions of high matrix porosity/permeability) should have similar effect on all azimuthal responses whereas linear, open fracture trends will produce a response that varies azimuthally.

In order to test this hypothesis, four azimuth-limited seismic volumes were developed from the Baseline 3-D survey. The geometry of these azimuthal surveys is shown below in Figure 76. Seismic horizon interpretation was performed for each of these azimuthal volumes. In order to minimize any discrepancies that might have occurred during the processing of the individual azimuthal volumes and to adjust for any variation in the velocity analysis performed during processing all four azimuthal volumes were flattened on the A2 Carbonate reflection event. Isofrequency volumes were then extracted from the azimuthal flattened volumes. Based on the frequency analysis previously performed (see Figure 42) on the full azimuth volume I decided to focus the analysis on seven frequencies: $75 \mathrm{~Hz}, 65 \mathrm{~Hz}, 55 \mathrm{~Hz}, 45 \mathrm{~Hz}, 35 \mathrm{~Hz}, 25 \mathrm{~Hz}$ and $15 \mathrm{~Hz}$. The seismic volume was initially partitioned into 4 variable azimuth volumes (also referred to in the industry as "sectors"). These volumes incorporated data from shot / receiver pairs that were limited to specific azimuth ranges. The central azimuth is used to distinguish between the individual sectors. The geometry of these four sectors is listed here in Table 1 and illustrated in Figure 76.

Table 1: Geometry of the four azimuthal volumes developed from the Baseline survey.

\begin{tabular}{|c|c|c|}
\hline Sector Name & Azimuthal Range & Central Azimuth \\
\hline Azimuth 1 & 2.5 to 47.5 degrees & $25^{\circ}$ \\
\hline Azimuth 2 & 47.5 to 92.5 degrees & $70^{\circ}$ \\
\hline Azimuth 3 & 92.5 to 137.5 degrees & $115^{\circ}$ \\
\hline Azimuth 4 & 137.5 to 182.5 degrees & $160^{\circ}$ \\
\hline
\end{tabular}




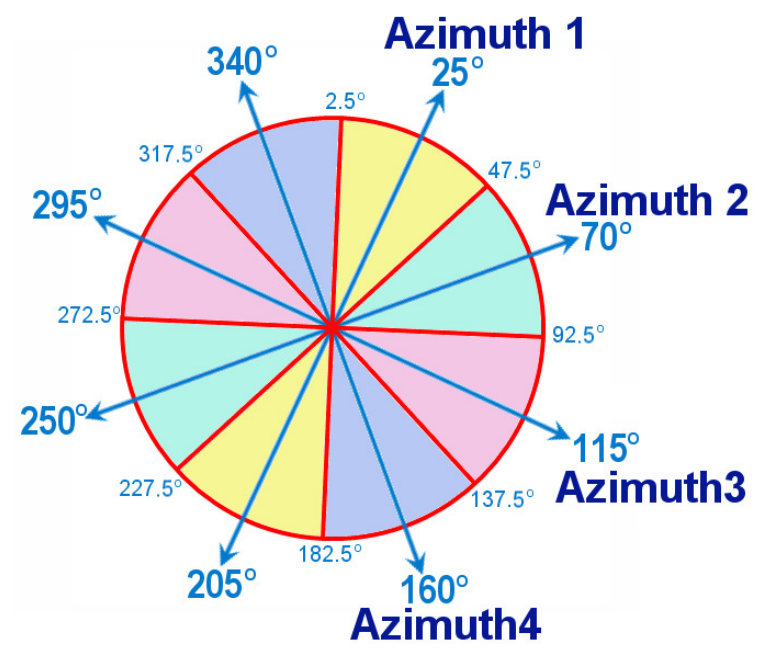

Figure 76: Sector geometries along which the Charlton 30/31 azimuthal seismic volumes were developed.

As a result of extracting the 7 iso-frequency volumes listed above, $(75 \mathrm{~Hz}, 65 \mathrm{~Hz} \ldots 15 \mathrm{~Hz})$, from these 4 azimuthal volumes (sectors) was the development of 28 separate azimuthal isofrequency volumes. These individual iso-frequency volumes were examined and interpreted during this study, the results of which are reported later in this report. These 28 individual iso-frequency volumes are shown in Appendix $\mathrm{C}$ and in Table 2 below along the designations for the summation volumes created during two normalization processes, which are discussed in the next section.

Table 2: Basic iso-frequency volumes separated by azimuth. The abbreviations indicate frequency and azimuthal range. For example: IF15A25 corresponds to the $15 \mathrm{~Hz}$. iso-frequency volume for azimuthal range 1 which has a central frequency of 25 degrees. Summed iso-frequency volumes (constant azimuth) are listed in the bottom row and summed azimuthal volumes (constant frequency) are listed in the last column.

\begin{tabular}{|c|c|c|c|c|c|}
\hline Frequency & $\begin{array}{c}\text { Azimuth } 1 \\
\left(25^{\circ}\right)\end{array}$ & $\begin{array}{c}\text { Azimuth } 2 \\
\left(7^{\circ}\right)\end{array}$ & $\begin{array}{c}\text { Azimuth } 3 \\
\left(1_{15}\right)^{\circ}\end{array}$ & $\begin{array}{c}\text { Azimuth } 4 \\
\left(160^{\circ}\right)\end{array}$ & $\begin{array}{c}\text { All Azimuth } \\
\text { Fraction Sum }\end{array}$ \\
\hline $15 \mathrm{~Hz}$ & IF15A25 & IF15A70 & IF15A115 & IF15A160 & AAF15Sum \\
\hline $25 \mathrm{~Hz}$ & IF $25 \mathrm{~A} 25$ & IF25A70 & IF25A115 & IF25A160 & AAF25Sum \\
\hline $35 \mathrm{~Hz}$ & IF35A25 & IF35A70 & IF35A115 & IF35A160 & AAF35Sum \\
\hline $45 \mathrm{~Hz}$ & IF45A25 & IF45A70 & IF45A115 & IF45A160 & AAF45Sum \\
\hline $55 \mathrm{~Hz}$ & IF55A25 & IF55A70 & IF55A115 & IF55A160 & AAF55Sum \\
\hline $65 \mathrm{~Hz}$ & IF65A25 & IF65A70 & IF65A115 & IF65A160 & AAF65Sum \\
\hline $75 \mathrm{~Hz}$ & IF75A25 & IF75A70 & IF75A115 & IF75A160 & AAF75Sum \\
\hline $\begin{array}{c}\text { Selected Frequencies } \\
\text { Fraction Sum }\end{array}$ & SFFA25Sum & SFFA70Sum & SFFA115Sum & SFFA160Sum & \\
\hline
\end{tabular}




\subsubsection{Normalization of Iso-Frequency Volumes}

In addition to interpreting the individual iso-frequency volumes comparisons were also performed on normalized volumes. In order to facilitate relative comparison the 4 volumes associated with individual frequencies were normalized resulting in amplitude volumes that could be related to one another by percentage. To do this, summed volumes were created by adding all seven isofrequency volumes sharing a single azimuth (as an example: IF15A25 through IF75A25 in Table 2). Normalized volumes were calculated by dividing each of the iso-frequency volumes by the corresponding sum volume. This produced 28 iso-frequency volumes with amplitude values that were percentages of the Sum volumes for each azimuth. Symbolically, these normalized volumes were calculated as follows:

$$
S F F V_{i}=\frac{I F V_{i}}{\sum_{f=1}^{7} I F V_{f}}
$$

where $\mathbf{S F F V}_{\mathrm{i}}$ refers to the Selected Frequency Fraction Volume, IFV to IsoFrequency Volume, $\mathrm{f}$ to frequencies 1 through 7 and I to individual normalized frequencies and i represents 15 Hz, 25Hz., etc. Figure 77 illustrates this normalization.

All 7 iso-frequency volumes for a single azimuth were added together to create a single, azimuthal sum volume. Each of the 7 iso-frequency volumes was then divided by the azimuthal sum volume to produce an azimuthal normalized (or ratio) iso-frequency volume. Since only certain frequencies were used in this analysis and not the entire frequency content of the seismic data these are referred to as Selected Frequencies Fraction (SFF) Volumes. 


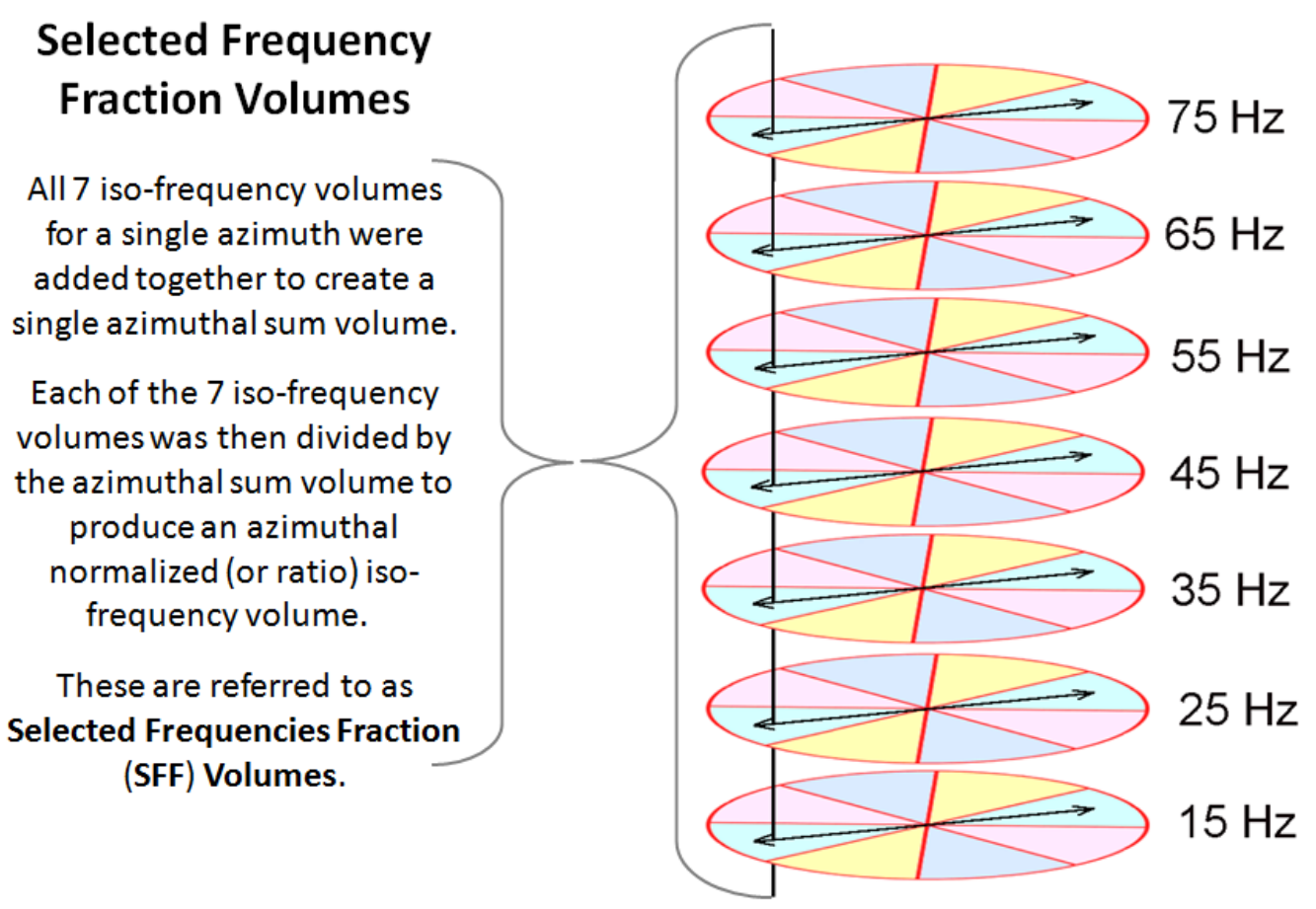

Figure 77: Normalization of the iso-frequency volumes in specific azimuths to produce the Selected Frequency Fraction (SFF) Volumes.

Screen captures showing a selected in-line and cross-line for each of these four azimuthal isofrequency sum volumes are shown in Appendix D. Next each azimuthal iso-frequency volume was divided by the azimuthal sum volume in order to produce the Selected Frequencies Fraction (SFF) Volumes. The seven Selected Frequencies Fraction (SFF) Volumes would allow the amplitudes in each volume to be compared with the others within the same azimuth. The total of these would equal 100\% and as a quality control step all seven Selected Frequencies Fraction (SFF) Volumes within each azimuth were summed together. For each azimuth this step produced volumes that summed to 100\%. These are documented in Appendix E. The Selected Frequencies Fraction (SFF) Volumes were then interpreted. This interpretation was performed primarily using time slices taken through the Selected Frequencies Fraction (SFF) Volumes. A number of features believed to be related to geologic features and some artifacts were observed and are reported in the next section. A quality control check was performed by adding the Selected Frequency Fraction Volumes together. When performed these should have produced a quality control volume where all of the sample values 
equaled $100 \%$. During this quality control check an error was identified which had occurred during the processing of azimuth $25^{\circ}$. These volumes were regenerated and re-checked and then found to be correct. Table 3 shows these 28 azimuthal iso-frequency volumes and their designations used from this point forward in this report and their sum volumes.

Table 3: Iso-frequency volumes normalized by frequency. The abbreviations indicate frequency and azimuthal range. For example: SFF15A25 corresponds to the $15 \mathrm{~Hz}$. iso-frequency volume for azimuthal range 1 which has a central frequency of 25 degrees. These iso-frequency volumes have been normalized with regard to all iso-frequency volumes (that were developed for selected frequencies) within the same azimuth.

\begin{tabular}{|c|c|c|c|c|}
\hline Frequency & $\begin{array}{l}\text { Azimuth } 1 \\
\left(25^{\circ}\right)\end{array}$ & $\begin{array}{c}\text { Azimuth } 2 \\
\left(70^{\circ}\right)\end{array}$ & $\begin{array}{c}\text { Azimuth } 3 \\
\left(115^{\circ}\right)\end{array}$ & $\begin{array}{c}\text { Azimuth } 4 \\
\left(160^{\circ}\right)\end{array}$ \\
\hline $15 \mathrm{~Hz}$ & SFF15A25 & SFF15A70 & SFF15A115 & SFF15A160 \\
\hline $25 \mathrm{~Hz}$ & SFF25A25 & SFF25A70 & SFF25A115 & SFF25A160 \\
\hline $35 \mathrm{~Hz}$ & SFF35A25 & SFF35A70 & SFF35A115 & SFF35A160 \\
\hline $45 \mathrm{~Hz}$ & SFF45A25 & SFF45A70 & SFF45A115 & SFF45A160 \\
\hline $55 \mathrm{~Hz}$ & SFF55A25 & SFF55A70 & SFF55A115 & SFF55A160 \\
\hline $65 \mathrm{~Hz}$ & SFF65A25 & SFF65A70 & SFF65A115 & SFF65A160 \\
\hline $75 \mathrm{~Hz}$ & SFF75A25 & SFF75A70 & SFF75A115 & SFF75A160 \\
\hline
\end{tabular}

These Selected Frequency Fraction Volumes were then examined and interpreted in order to identify where frequency attenuation was occurring. This portion of the analysis was focused on identifying attenuation within each of the four azimuths. The results of this interpretation are discussed in the next chapter.

Additionally, normalization was performed for specific iso-frequency volumes. In this method the $15 \mathrm{~Hz}$ iso-frequency volumes from all four azimuths were summed together and then divided by the sum. Mathematically this operation is expressed as

$$
A A F V_{f}=\frac{I F V_{f}}{\sum_{i=1}^{4} I F V_{i}}
$$


where $\mathbf{A A F V}$ fefers to the All Azimuth Frequency Volumes, for frequency f, IFV $_{\mathrm{f}}$ to an individual Iso-Frequency Volume for frequency $\mathrm{f}$, and $\mathrm{IFV}_{\mathrm{i}}$ to the Iso-Frequency Volume for azimuth i. This produced iso-frequency normalized volumes which could then be compared to one another. Figure 78 illustrates how these volumes were normalized.

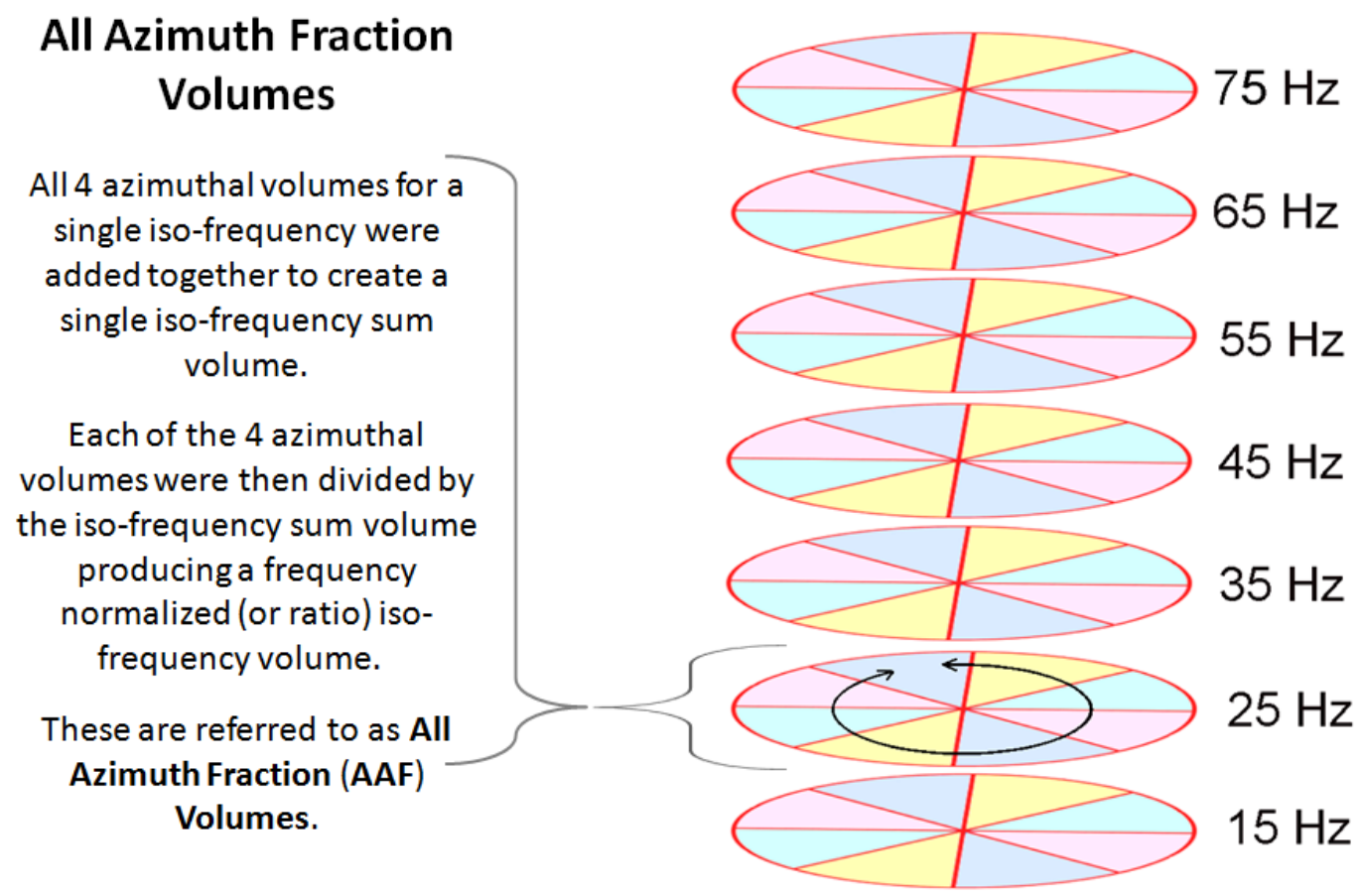

Figure 78: Normalization of the iso-frequency volumes of a specific frequency to produce the All Azimuth Fraction (AAF) Volumes.

It should be noted that these normalized volumes are substantially different from one another.

The Selected Frequency Fraction Volumes are normalized within a specific azimuth direction across the seven selected frequencies, whereas the All Azimuth Frequency Volumes are normalized within a specific frequency across all four azimuths. Table 2 shows the relationship between these two different normalization methods. 
Table 4: Iso-frequency volumes normalized by azimuth. The abbreviations indicate frequency and azimuthal range. For example: AAF15A25 corresponds to the $15 \mathrm{~Hz}$. iso-frequency volume for azimuthal range 1 which has a central frequency of 25 degrees. These iso-frequency volumes have been normalized with regard to all iso-frequency volumes within the same frequency.

\begin{tabular}{|c|c|c|c|c|}
\hline Frequency & $\begin{array}{c}\text { Azimuth } 1 \\
\left(25^{\circ}\right)\end{array}$ & $\begin{array}{c}\text { Azimuth } 2 \\
\left(\mathbf{7 0}^{\mathbf{o}}\right)\end{array}$ & $\begin{array}{c}\text { Azimuth } 3 \\
\left(115^{\circ}\right)\end{array}$ & $\begin{array}{c}\text { Azimuth } 4 \\
\left(160^{\circ}\right)\end{array}$ \\
\hline $15 \mathrm{~Hz}$ & AAF15A25 & AAF15A70 & AAF15A115 & AAF15A160 \\
\hline $25 \mathrm{~Hz}$ & AAF25A25 & AAF25A70 & AAF25A115 & AAF25A160 \\
\hline $35 \mathrm{~Hz}$ & AAF35A25 & AAF35A70 & AAF35A115 & AAF35A160 \\
\hline $45 \mathrm{~Hz}$ & AAF45A25 & AAF45A70 & AAF45A115 & AAF45A160 \\
\hline $55 \mathrm{~Hz}$ & AAF55A25 & AAF55A70 & AAF55A115 & AAF55A160 \\
\hline $65 \mathrm{~Hz}$ & AAF65A25 & AAF65A70 & AAF65A115 & AAF65A160 \\
\hline $75 \mathrm{~Hz}$ & AAF75A25 & AAF75A70 & AAF75A115 & AAF75A160 \\
\hline
\end{tabular}




\section{CHAPTER 6 - AZIMUTHAL ISO-FREQUENCY INTERPRETATION}

\subsection{Introduction}

In the preceding chapters, a relationship was proposed that zones of low instantaneous frequency corresponded to drained, high flow zones within the reef reservoir complex. Evidence supporting this relationship included: 1) a significantly negative correlation between increases of porosity with decreases of instantaneous frequency (Figure 50); 2) coincidence between zones of lower instantaneous frequency and production/perforation zones within the field's boreholes, 3) history matched reservoir simulation based on field scale porosity distribution derived using this relationship; and 4) coincidence between increased amplitudes in the 4D Monitor survey with previously identified high porosity zones.

In this chapter a new and novel analytical technique for identifying zones of frequency attenuation is investigated. This method employs the use of azimuthal iso-frequency analysis and is significantly different from the analysis presented in chapters 2-4. The data sets used in this part of the study are described and tabulated in Chapter 5. Recall that these volumes were all flattened on the A2 Carbonate reflector, which is the strongest seismic event in the vicinity of the reef and immediately above the reef (see Figure 79, shown below). Additionally, the analysis concentrates on the northern portion of the reef where the enhanced oil recovery operations were conducted and any evidence of $\mathrm{CO}_{2}$ movement within the reservoir would be observed utilizing the 4D seismic. As a consequence of the flattening of the seismic the data, the two-way-traveltime to the A2 Carbonate reflector represents zero time for each interpreted azimuthal volume. Figure 79 shows a normal and flattened in-line (1073) from azimuth 4, (central azimuth $160^{\circ}$ ). 

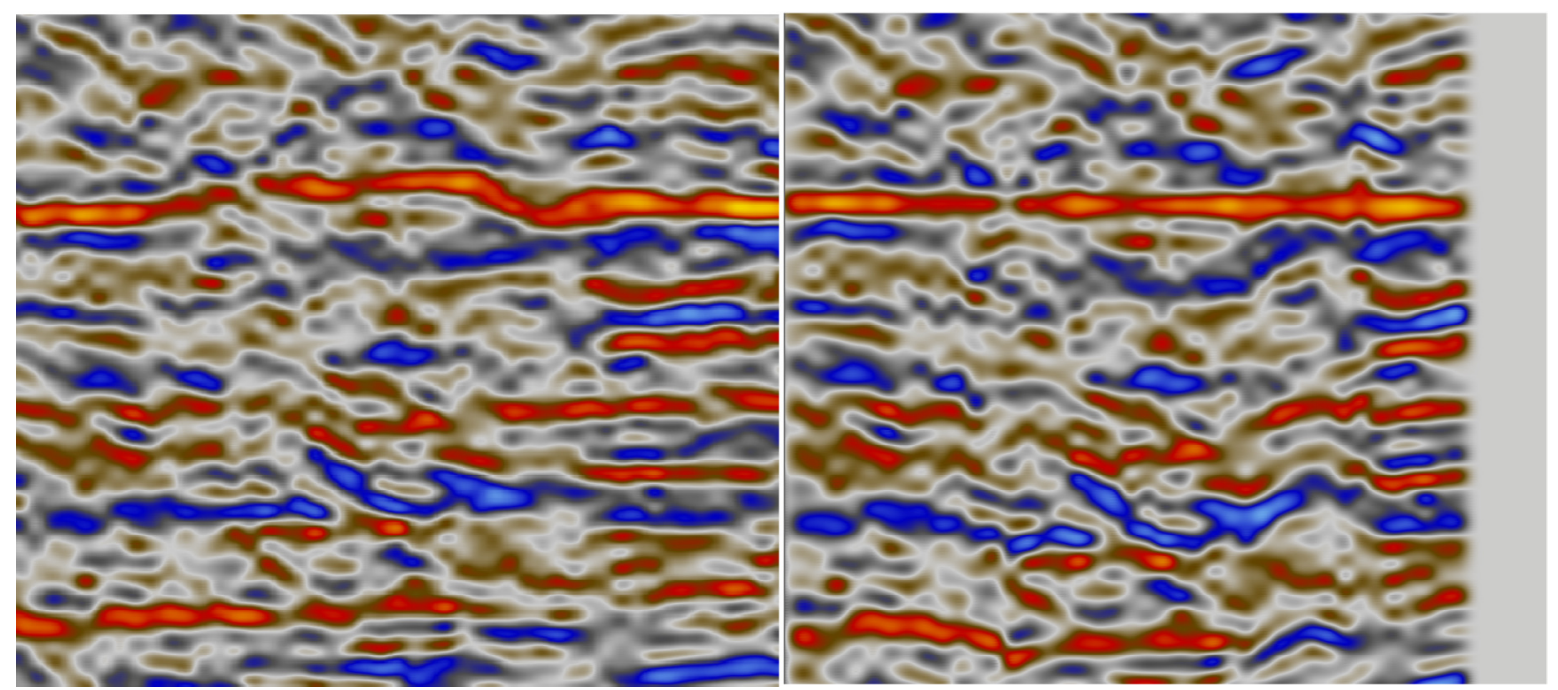

Figure 79: Normal and flattened in-line 1073 for the $160^{\circ}$ azimuthal seismic volumes.

The borehole position for the deviated borehole within the field, the State Charlton "C" 2-30, has been adjusted on the various time slices presented later in this chapter to show it entry location into the flattened A2Carbonate. Since all boreholes in the field with this one exception were drilled vertically their borehole positions in map view were unaffected by the flattening. However, since the State Charlton "C" 2-30 well is the $\mathrm{CO}_{2}$ injector well, it is important to understand its borehole position within of the field with respect to potential seismic anomalies. The deviation survey for this borehole was truncated above the top of the A2 Carbonate and this point was set to time 0 milliseconds in all subsequent time slices. Accordingly, on maps shown later in this thesis only the northern portion of the borehole is shown. The borehole position shown on the flattened seismic data is labeled "Deep" to differentiate the deeper borehole penetration into the A2 carbonate from the surface borehole locations.

\subsection{Observations}

Given the large number of 3D seismic volumes generated for this study, it was decided to limit the analysis to the zones containing features discussed earlier in the study. As reported in earlier chapters seismic anomalies were observed on the monitor seismic survey in reservoir locations 
predicted by the forward reservoir simulation to be filled with injected $\mathrm{CO}_{2}$. These features occur at the top of the reservoir in the northern portion of field, as would be expected due to the CO2's buoyancy. This anomaly is indicated in white below in Figure 80.

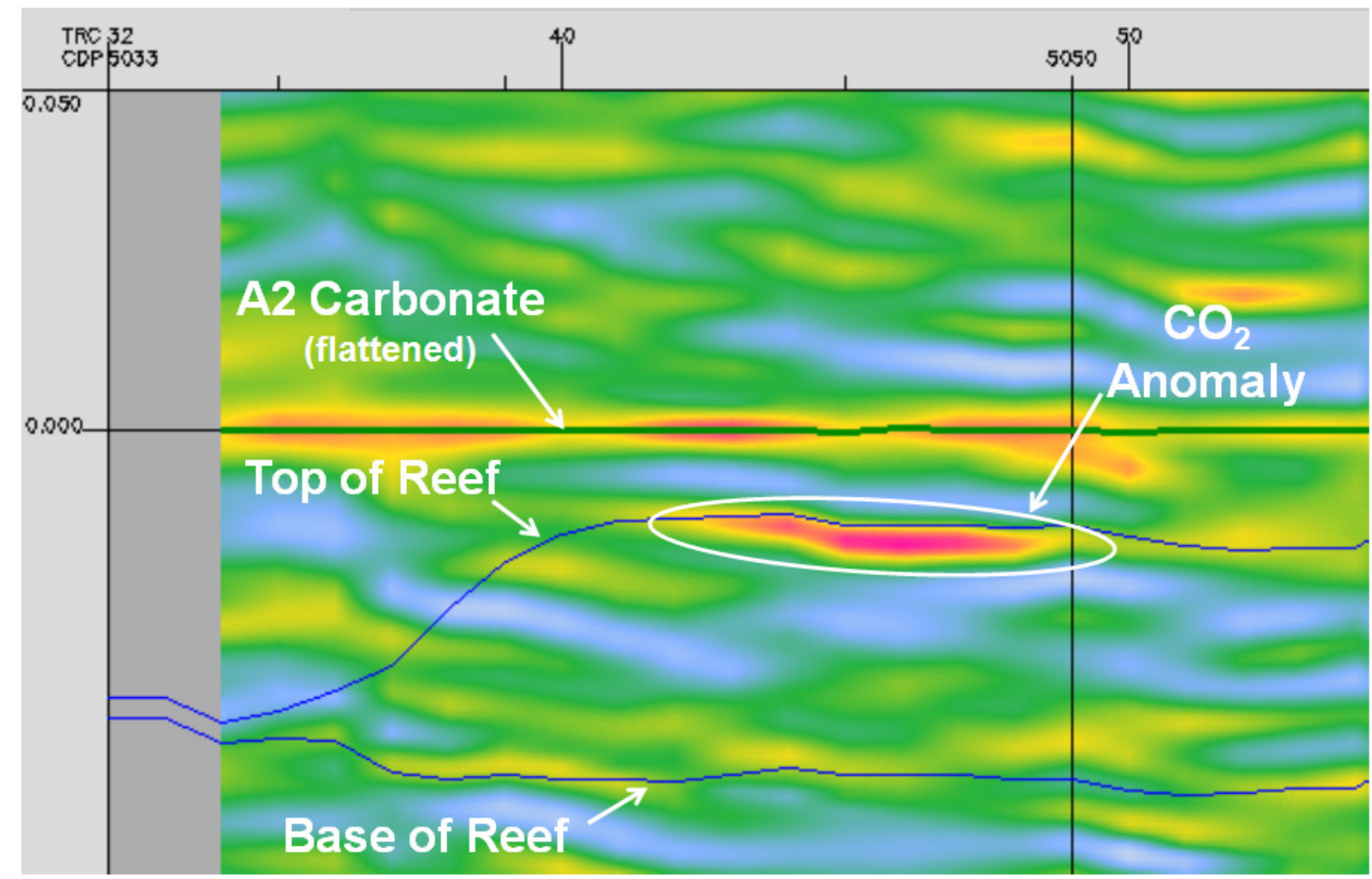

Figure 80: In-line 1072 through flattened A2 Carbonate volume from the monitor survey. The amplitude anomaly within the reservoir interval is located along the top of the reef, circled in white. The anomaly is interpreted to result from changes of impedance related to $\mathrm{CO} 2$ flooding of this interval. The top and base of the reef are shown as blue lines.

The comparison between the Baseline and Monitor surveys of the amplitudes from the A2 Carbonate reflector, reported in section 4.6 or Chapter 4 , indicate little to no variation in seismic signal due to acquisition and processing in the northern half of the field. Figure 81 shows this comparison and the focus area for this portion of the study which is shown in red. 


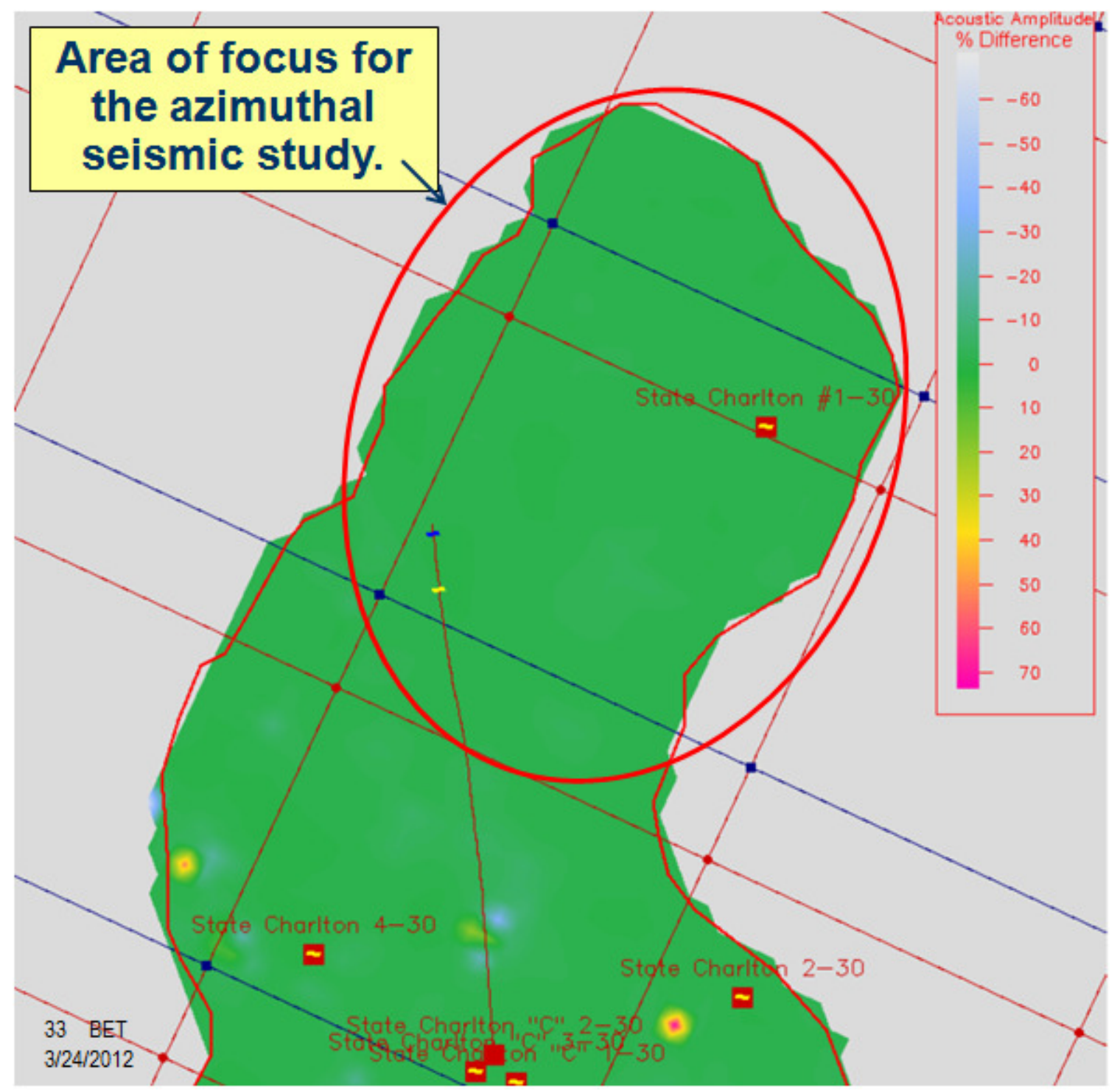

Figure 81: Difference in the amplitudes of the A2 Carbonate reflection event between the Baseline and Monitor surveys (Baseline -Monitor). Location of azimuthal seismic study is circled in red.

\subsubsection{Comparison of All Azimuth Fraction (AAF) and Azimuthal Iso-Frequency Volumes}

A visual comparison was preformed between the original azimuthal iso-frequency volumes and the All Azimuth Fraction (AAF) volumes (azimuthally normalized volumes). Figure 82 shows this comparison of time slice $15 \mathrm{~ms}$ (below the flattened A2 Carbonate reflector) between the original iso-frequency azimuthal volume for azimuth 1 (central azimuth $25^{\circ}$ ) on the left and the All Azimuth Fraction (AAF) volume on the right. In this figure, and the following figures that show data from the 
flattened seismic volumes, the boreholes positions are with respect to the flattened A2Carbonate which is time 0 (zero) in those seismic volumes. In these images the boreholes have the prefex "Deep_" added to them. Only minor differences were observed between the original iso-frequency azimuthal volume and the All Azimuth Fraction (AAF) volumes (azimuthally normalized volumes).

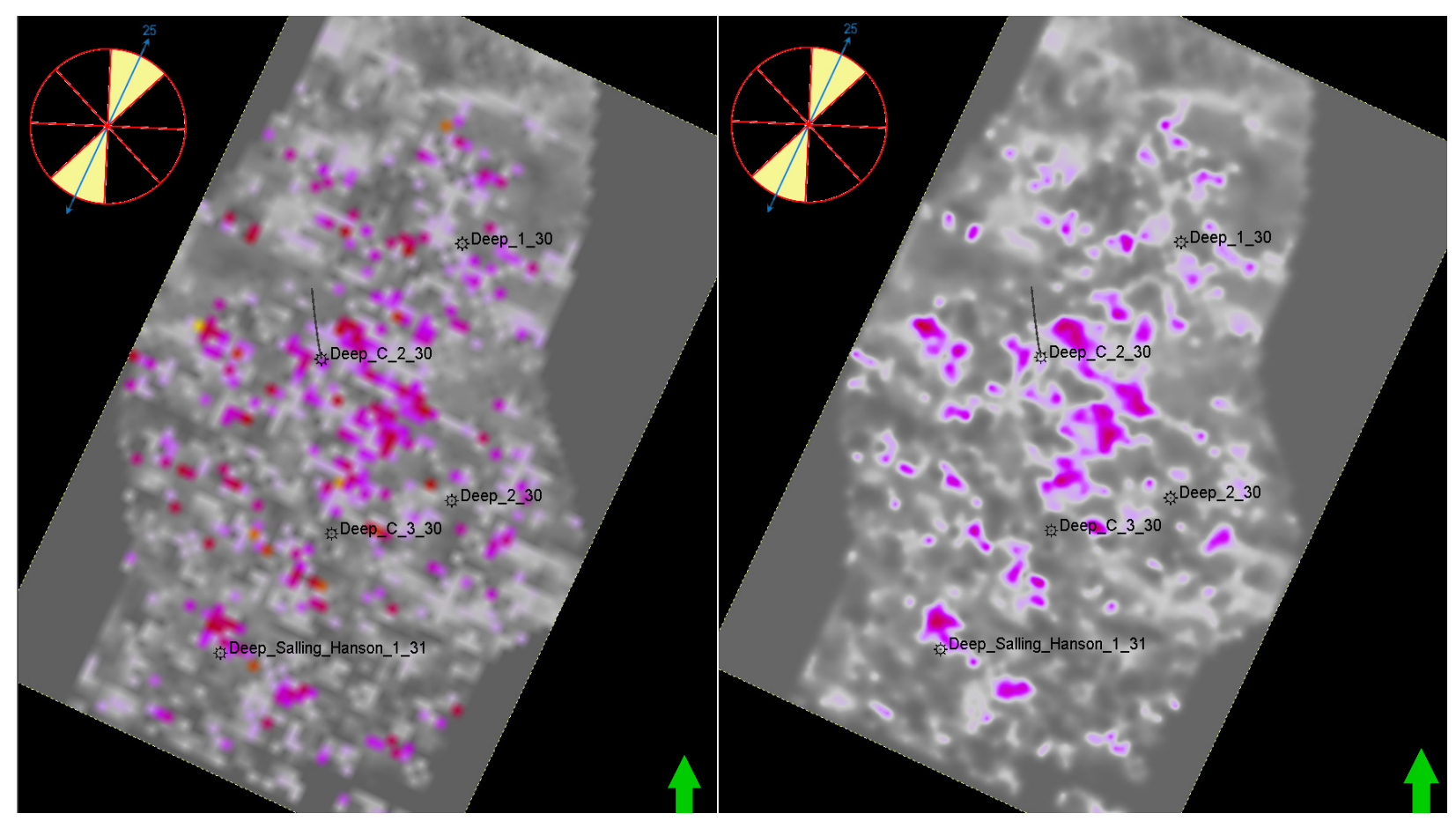

Figure 82: Time slice $15 \mathrm{~ms}$ below the A2 Carbonate event extracted from the $25 \mathrm{~Hz}$ volumes. The original azimuthal iso-frequency volume is shown on the left and the all Azimuth Fraction (AAF) volume is shown on the right.

\subsubsection{Single Azimuth (25) Iso-Frequency Responses}

The strongest amplitude response observed in the iso-frequency volumes occurs along IF55A25 (the $25^{\circ}$ azimuth in the $55 \mathrm{~Hz}$ iso-frequency volume) for time slice $15 \mathrm{~ms}$ below the flattened A2 Carbonate reflector. This azimuth also displays strong amplitudes in the $65 \mathrm{~Hz}$ (IF65A25) and $75 \mathrm{~Hz}$ (IF75A25) iso-frequency volumes. The $45 \mathrm{~Hz}$. Iso-frequency volume (IF45A25) shows a significant drop in strength throughout the survey, as does the $35 \mathrm{~Hz}$ isofrequency volume (IF35A25). However, $25 \mathrm{~Hz}$ (IF25A25) and $15 \mathrm{~Hz}$ (IF15A25) on the single- 
azimuth $\left(25^{\circ}\right)$ iso-frequency volumes show some strong amplitudes in various isolated localities (see Figure 83). These strong amplitudes occur at the same locations on the time slices.

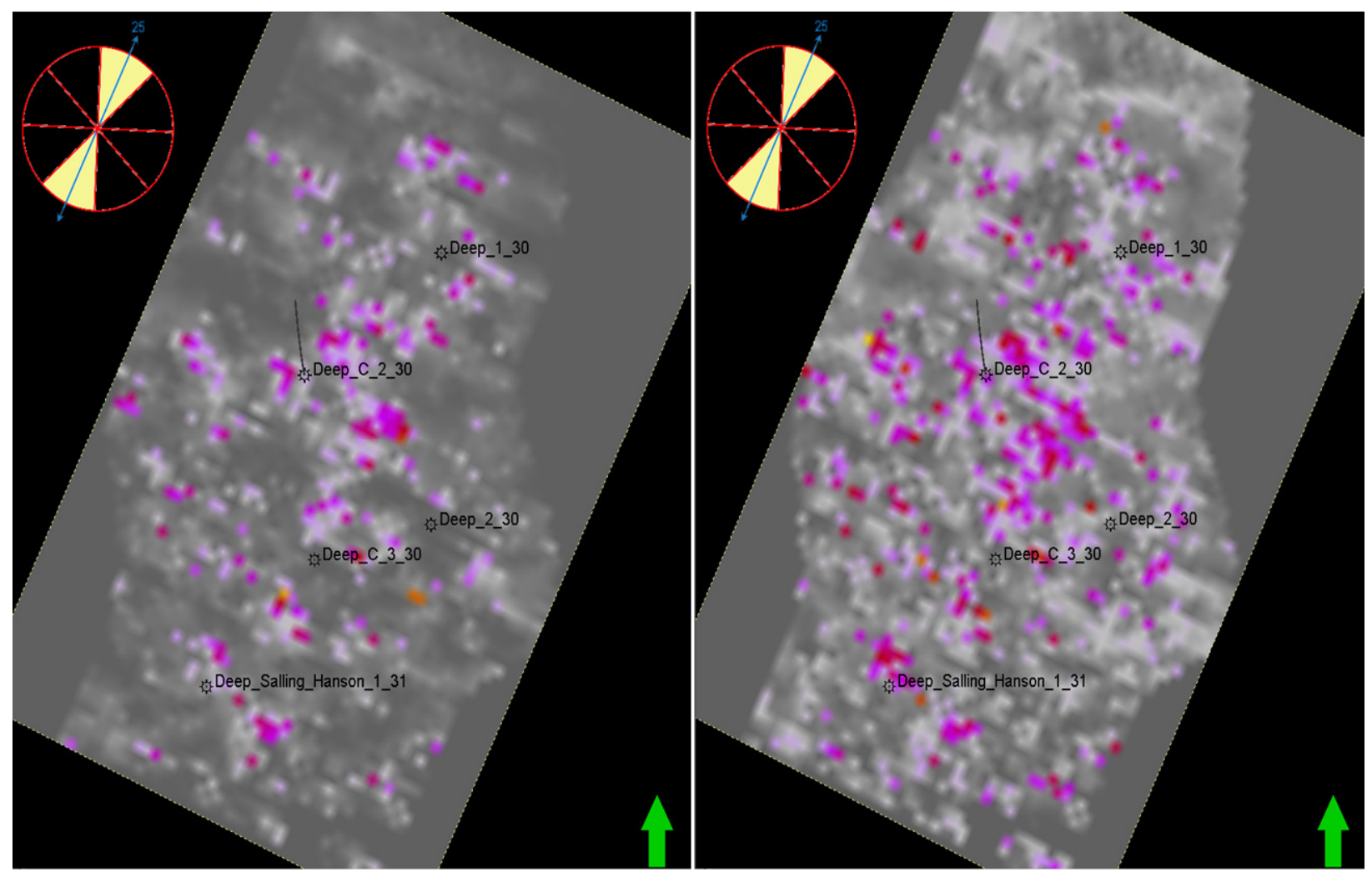

Figure 83: Time slice $15 \mathrm{~ms}$ below the A2 Carbonate event extracted from the single azimuth $\left(25^{\circ}\right) 15 \mathrm{~Hz}$ iso-frequency (IF15A25) on left and the $25 \mathrm{~Hz}$ iso-frequency (IF25A25) on right.

\subsubsection{Single Azimuth (70) Iso-Frequency Responses}

The $70^{\circ}$ azimuth contains the highest amplitudes at $65 \mathrm{~Hz}$. Note that during this comparison the Selected Frequency Fraction volumes were examined instead of the original iso-frequency volumes. As mentioned previously little difference between volumes normalized in this manner and the original volumes had been observed. As in the previous comparisons the amplitudes are examined $15 \mathrm{~ms}$ below the A2 Carbonate. Figure 84 shows a comparison of the Selected Frequency Fraction volumes for Azimuth 2 (central azimuth 70 ) for volumes $65 \mathrm{~Hz}$ (SFF65A70) through $15 \mathrm{~Hz}$ 
(SFF15A70). The $55 \mathrm{~Hz}$ (SFF55A70), $45 \mathrm{~Hz}$ (SFF45A70) and $35 \mathrm{~Hz}$ (SFF35A70) time slices display a progressive decrease of amplitude from high to low frequency.

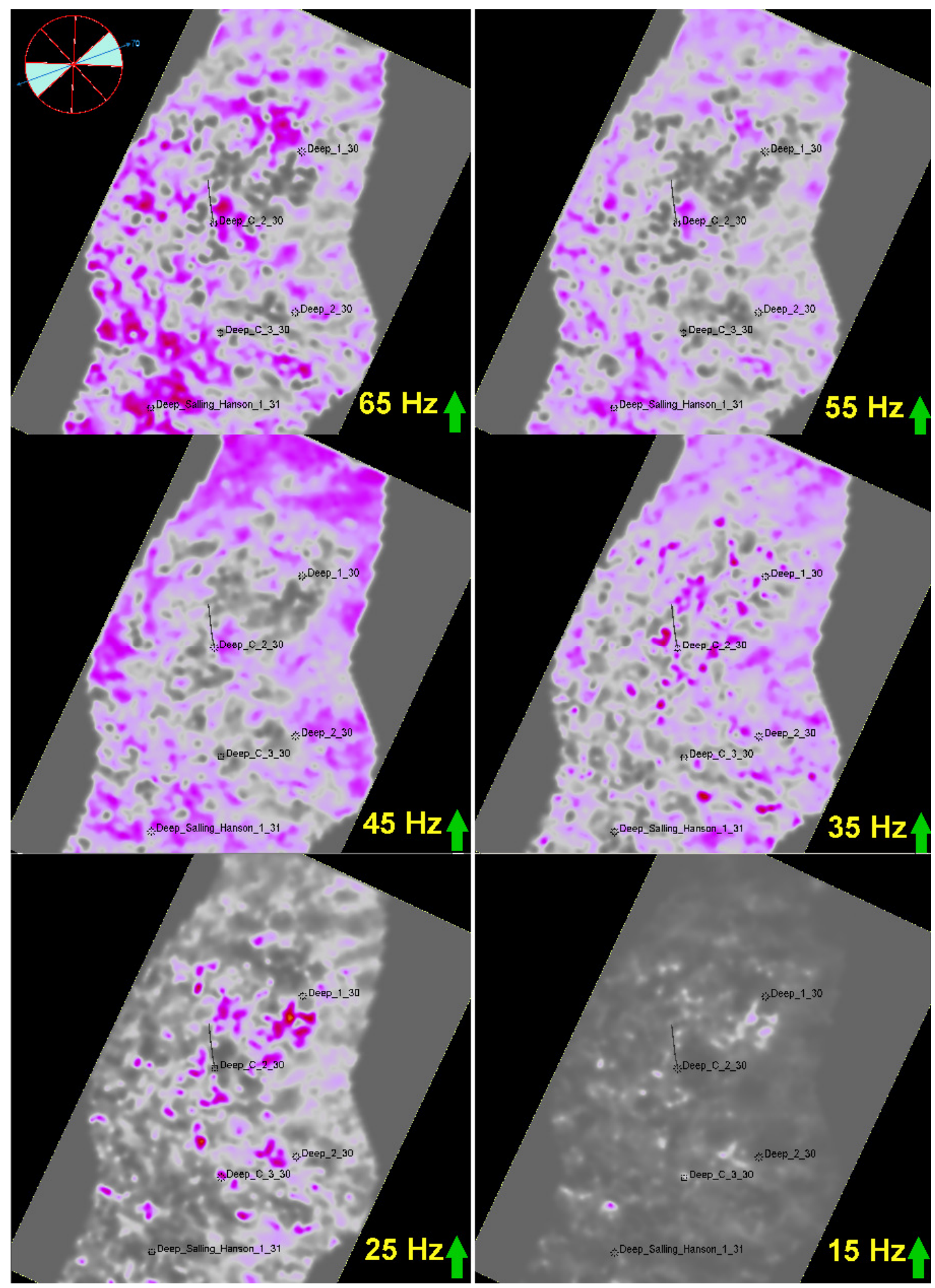

Figure 84: A comparison of the Selected Frequency Fraction iso-frequency volumes $65 \mathrm{~Hz}$ (SFF65A70) to $15 \mathrm{~Hz}$ (SFF15A70) in $10 \mathrm{~Hz}$. intervals for the $70^{\circ}$ azimuth, 
As with the $25^{\circ}$ azimuth, the $25 \mathrm{~Hz}$ iso-frequency volume for $70^{\circ}$ azimuth (SFF25A70) displays an overall background of low amplitudes with high amplitudes occurring in various isolated localities. The $15 \mathrm{~Hz}$ iso-frequency volume for Azimuth $70^{\circ}$ (SFF15A70) displays the same overall background of low amplitudes with stronger amplitudes occurring in various isolated localities that are seen on the $25 \mathrm{~Hz}$ iso-frequency volume and they occur in the same locations. However, the strength of these isolated strong amplitudes is significantly lower on the $15 \mathrm{~Hz}$ volume. Of significance is the observation that the locations of high amplitude regions in the $25 \mathrm{~Hz}$ and $15 \mathrm{~Hz}$ volumes are not coincident between $25^{\circ}$ and $70^{\circ}$ azimuths on the $15 \mathrm{~ms}$ time slice. This indicates that the strong low frequencies observed on azimuth $25^{\circ}$ are not related to tuning. Zones of tuning would affect the seismic signal from all directions and would be observed in comparisons on the appropriate azimuthal volumes. Comparison of the $15 \mathrm{~Hz}$ Iso-frequency volumes for azimuths 25 and 70 (see Figures 83 and 84 ) show that amplitudes are significantly stronger on azimuth 25 . Since these strong amplitudes are clearly directionally related the phenomenon observed cannot be related to tuning.

\subsubsection{Iso-Frequency comparisons along the $115^{\circ}$ and $160^{\circ}$ Azimuths}

The comparison described above was repeated for azimuths $115^{\circ}$ and $160^{\circ}$. Azimuth $115^{\circ}$ displays the strongest amplitudes on the $55 \mathrm{~Hz}$ iso-frequency volume. Iso-frequency volumes $35 \mathrm{~Hz}$, $25 \mathrm{~Hz}$ and $15 \mathrm{~Hz}$ show an overall decrease in amplitudes. In azimuth $160^{\circ}$ a steady decrease in strength is observed from $55 \mathrm{~Hz}$ downward towards the lower frequencies. However, as observed in azimuth $25^{\circ}$ in the lower frequencies strong localized amplitudes are distributed at various locations in the reef, particularly near the injection point.

\subsubsection{Lineament Interpretation in All Azimuths}

On all azimuthal volumes lineaments are observed at various orientations in different locations within the volumes. Some of these are aligned with the survey's in-line and cross-line directions and are quite obviously associated with the acquisition footprint. The acquisition related 
lineaments are discussed in the following section of this chapter. These lineaments include amplitudes that are strong or weak compared to the background.

Lineaments that do not appear to be acquisition related are also observed in the seismic data. These lineaments are believed to have geological significance since their orientations do not coincide with the in-line and cross-line directions. Lineaments interpreted to be associated with acoustic heterogeneities in the reservoir are discussed in Chapter 7.

\subsection{Potential Acquisition Footprint Issues}

During the interpretation of the azimuthal $15 \mathrm{~Hz}$ iso-frequency volumes potential acquisition related features were noted. Figure 85 shows the iso-frequency normalized time slice $15 \mathrm{~ms}$ for isofrequency $15 \mathrm{~Hz}$, azimuth $25^{\circ}$ with and without potential acquisition footprint interpreted in blue. The observed lineations are parallel to the in-line, and in some cases the cross line, directions. These lineations were not readily observable on the other volumes developed during this study. These features were recognized and disregarded during the interpretation.

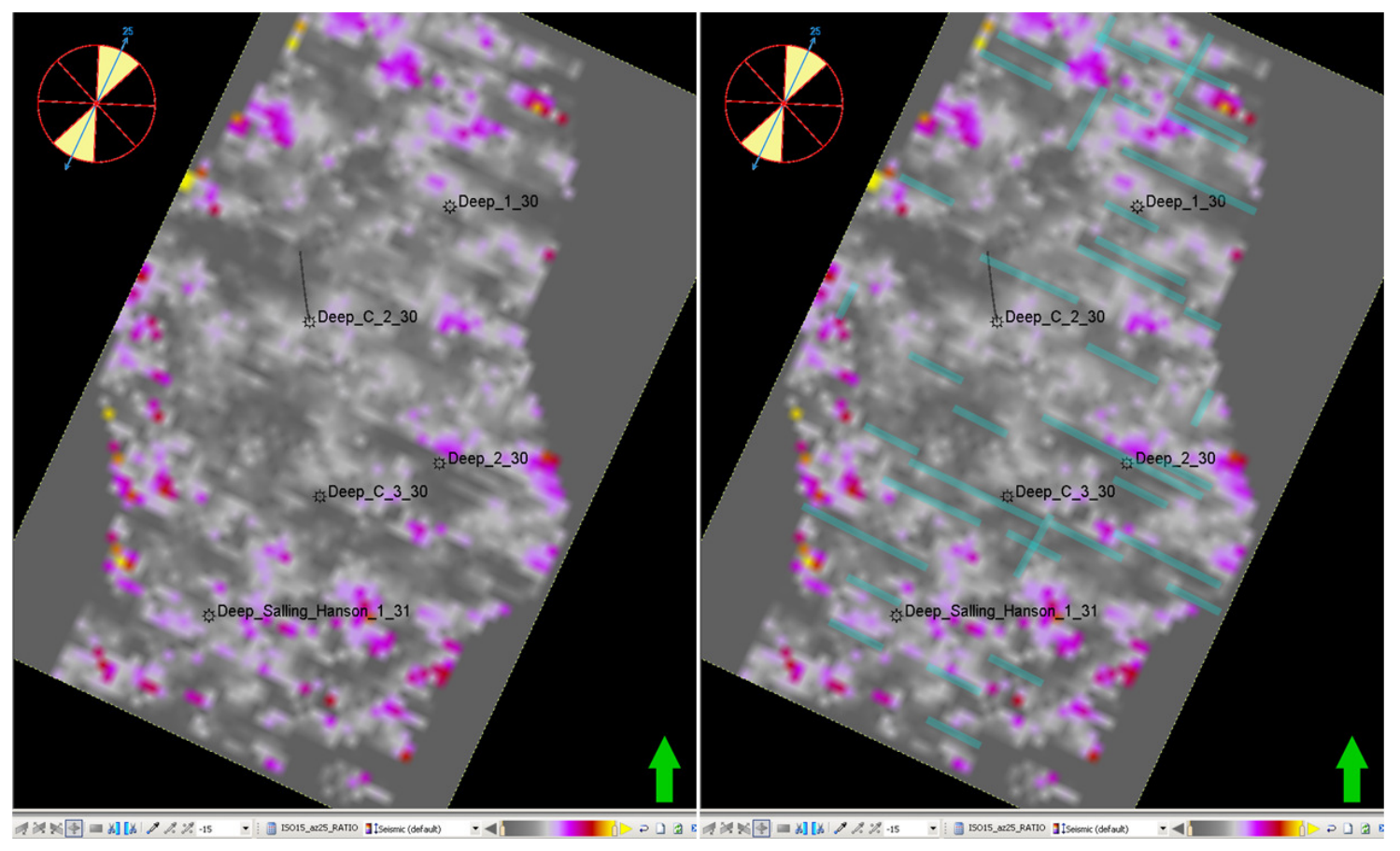

Figure 85: $15 \mathrm{~Hz}$ iso-frequency volume on azimuth $25^{\circ}$ at time slice $15 \mathrm{~ms}$, on the left and with acquisition footprint lineations interpreted on the right in blue. 


\subsection{Isotropic Frequency Attenuation}

All Azimuth Fraction (AAF) volumes were developed for $15 \mathrm{~Hz}$ and $55 \mathrm{~Hz}$ using all four azimuths. These volumes were extracted as described earlier in Chapter 5, (see Figure 78). During this process Iso-frequency Sum Volumes were developed by summing the amplitude from all four azimuths for a specific frequency. These Iso-frequency Sum Volumes were then compared. Figure 86 below shows significant contrast in frequency response between the $55 \mathrm{~Hz}$ All Azimuth Fraction Sum (AAF55Sum) and the $15 \mathrm{~Hz}$ All Azimuth Fraction Sum (AAF15Sum) volumes. The $55 \mathrm{~Hz}$ Isofrequency Sum Volume shows high amplitudes in yellow and red, (see color bar at top) primarily outside the reef whereas the $15 \mathrm{~Hz}$. Iso-frequency Sum Volume for the same time slice (15 ms below the flattened A2 Carbonate) shows relatively high amplitudes in the interior of the reef.

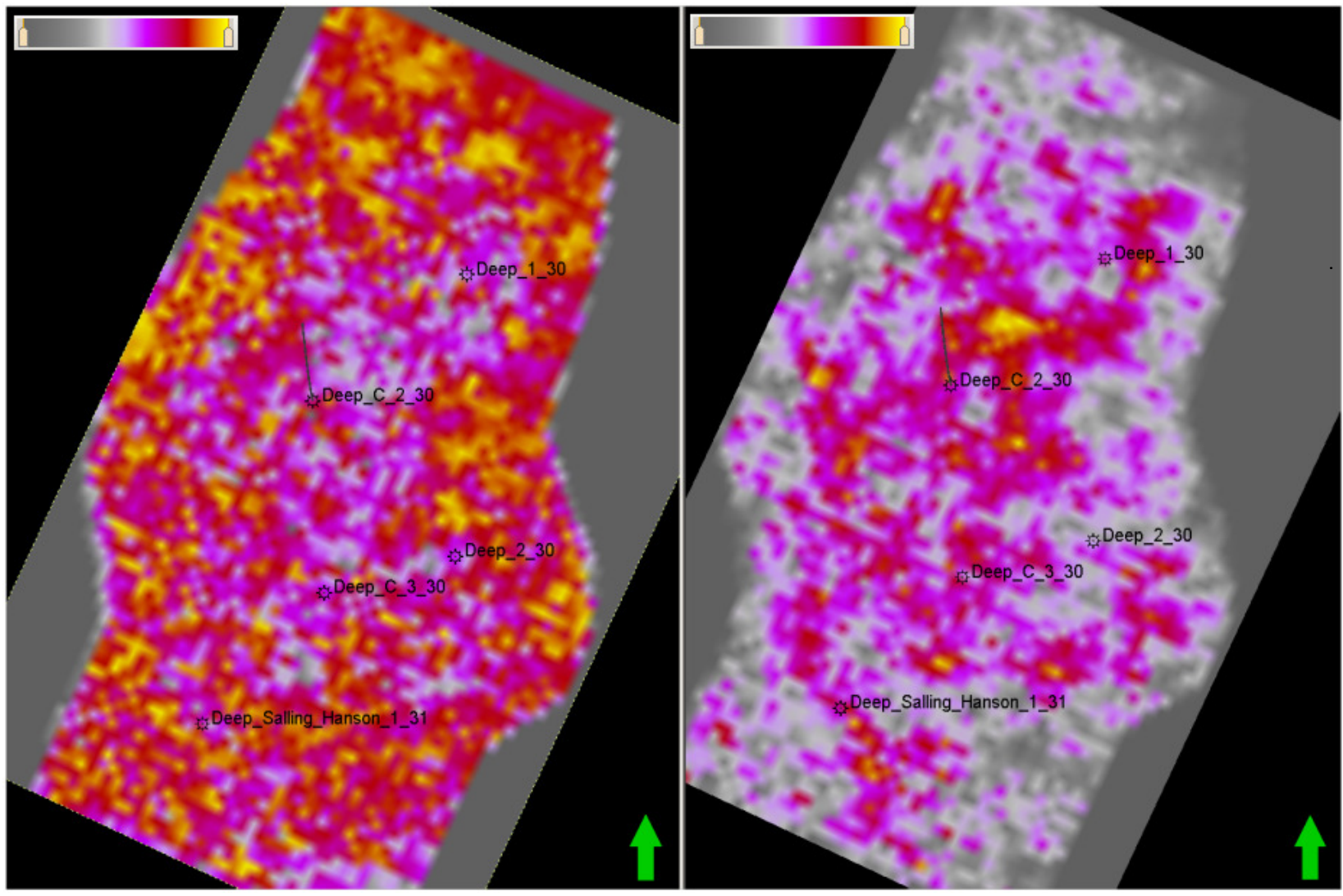

Figure 86: All Azimuth Fraction Sum Volumes developed during the construction of the All Azimuth Fraction (AAF) volumes for $55 \mathrm{~Hz}$ on left (AAF55Sum) and $15 \mathrm{~Hz}$ on right (AAF15Sum). 
This illustrates that frequency attenuation has occurred in the localities where the high amplitudes exist in the $15 \mathrm{~Hz}$ volume. Zones of low amplitude on the $15 \mathrm{~ms}$ time slice from the 55 $\mathrm{Hz}$ iso-frequency sum correspond to high amplitude zones from the $15 \mathrm{~Hz}$ iso-frequency sum. Odebeatu in 2006 used this same technique to detect frequency attenuation associated with a North Sea reservoir, see figure 87.
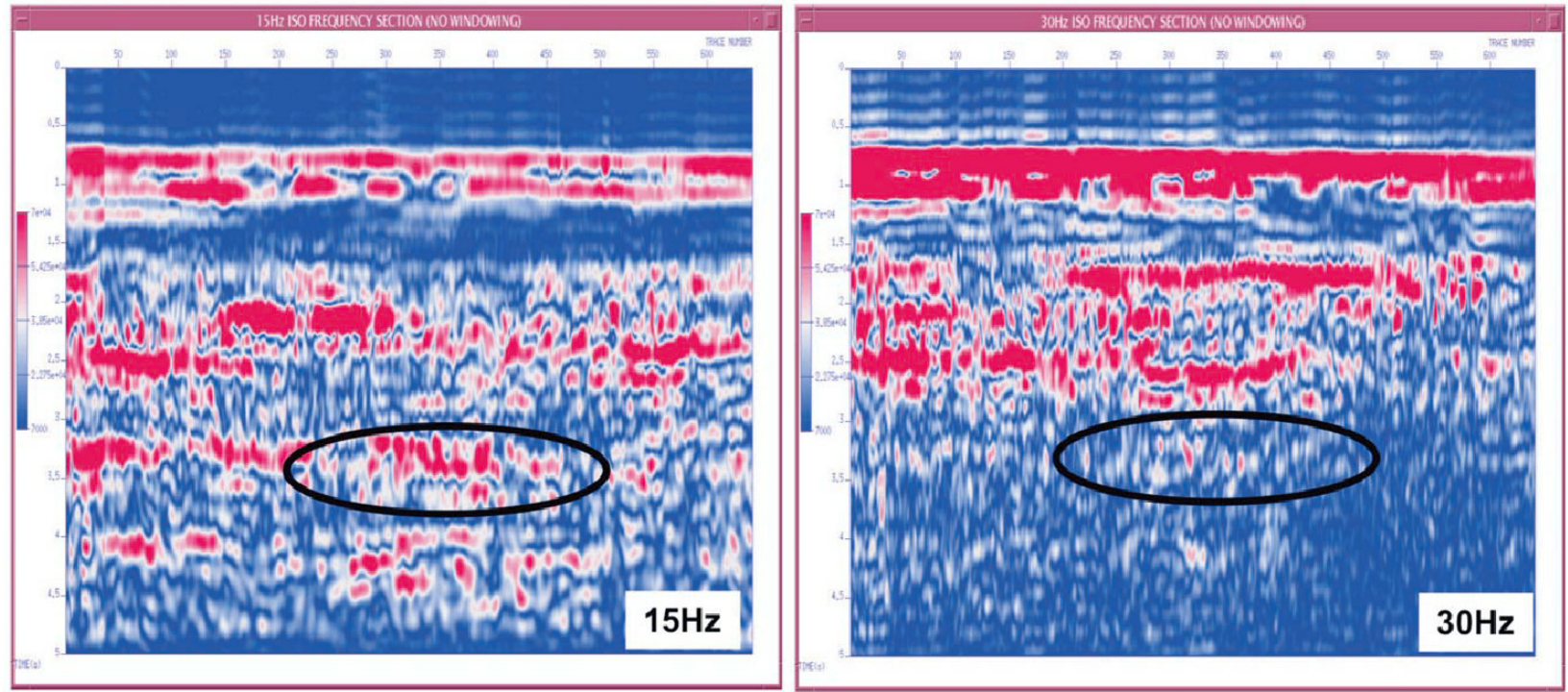

Figure 87: $15 \mathrm{~Hz}$ and $30 \mathrm{~Hz}$ iso-frequency sections used by Odebeatu (2006) to detect frequency attenuation associated with a reservoir in a gas field in the North Sea.

The time slice taken from the 15 to $55 \mathrm{~Hz}$ All Azimuth Fraction Sum Difference Volume 15 ms below the A2 Carbonate, shown in Figure 87. This difference volume was developed by subtracting the $15 \mathrm{~Hz}$ All Azimuth Fraction Sum volume from the $55 \mathrm{~Hz}$ All Azimuth Fraction Sum volume (both of which are displayed in Figure 86). 


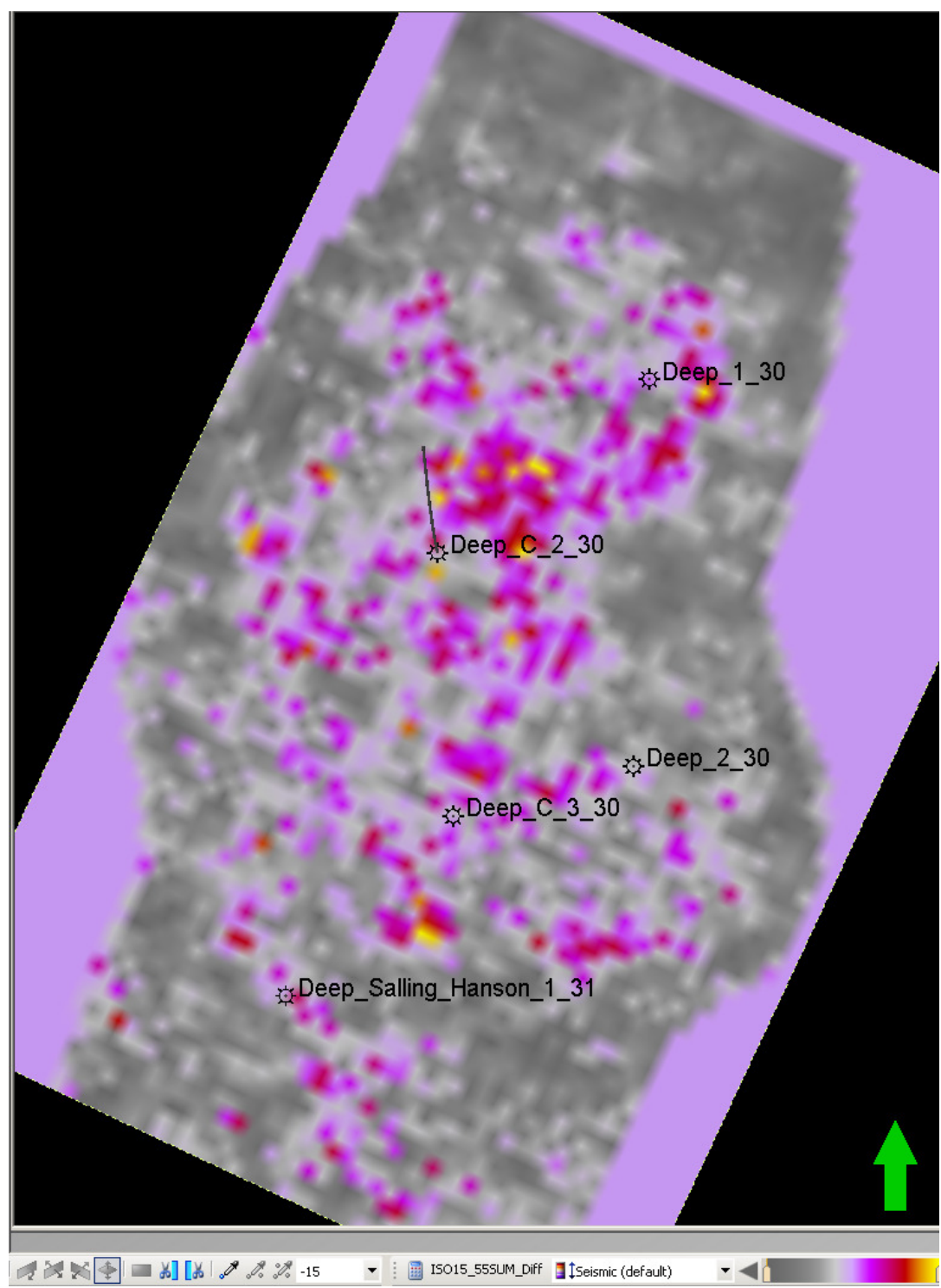

Figure 88: 15 to $55 \mathrm{~Hz}$ All Azimuth Fraction Sum Difference Volume $15 \mathrm{~ms}$ below the A2 Carbonate.

The main objective of this analysis was to illustrate the contrast between the two frequencies.

The $15 \mathrm{~Hz}$ All Azimuth Fraction Sum volume, which contained the higher amplitudes within the reef, had the $55 \mathrm{~Hz}$ All Azimuth Fraction Sum volume amplitudes subtracted from it in order to produce positive values to illustrate the difference between them. The $15 \mathrm{~Hz}$ All Azimuth Fraction Sum volume could have been subtracted from the $55 \mathrm{~Hz}$ All Azimuth Fraction Sum volume, producing negative difference values that illustrated the same amount of difference; however the color displays for this would have been reversed which would have complicated comparisons. The 
analytical methodology shown here is specifically designed to investigate the isotropic behavior of the reservoir. Since All Azimuth Fraction Sum Volumes were used in the analysis all azimuths were employed for this method. This would be similar in nature to analyzing the Iso-frequency volumes generated from the original full azimuth volume. The analysis reveals a buildup in low frequencies

along some but not all azimuths suggesting directional attenuation of high frequencies and not tuning, which would be observed on all azimuthal volumes.

\subsection{Isotropic Frequency Attenuation and Correlation with Previous Porosity Analyses}

Figures 86 and 88 show a number of events that are high amplitude in $15 \mathrm{~Hz}$ All Azimuth Fraction Sum Volume $15 \mathrm{~ms}$ below the A2 Carbonate. High amplitude regions observed at $15 \mathrm{~Hz}$. coincide with low amplitude regions in the $55 \mathrm{~Hz}$ Iso-Frequency Sum Volume. This correspondence is not exact. Drained, high flow systems within the reef are not uniformly distributed. The geometry of these zones along with associated porosity and permeability distributions will be quite variable. This could lead to variations in the extent of the frequency attenuation band (e.g. perhaps 30 to $55 \mathrm{~Hz}$ as opposed to 15 to $55 \mathrm{~Hz}$.). Therefore, a direct correlation between decreased $55 \mathrm{~Hz}$ and increased 15 Hz may not always be observed.

A number of these frequency anomalies correspond to locations between the $\mathrm{CO}_{2}$ injection point and the EOR producer that were predicted by reservoir simulation to be filled with $\mathrm{CO}_{2}$ following the injection of 29,000 tons of $\mathrm{CO}_{2}$ into the reservoir. This predictive reservoir simulation was based on the reservoir characterization developed using the instantaneous frequency to porosity relationship shown in Figure 50 and discussed previously in Chapter 4. Simulation grid cells (taken from layer 9 at time step Sept., 2007) that indicate areas of $\mathrm{CO}_{2}$ flooding predicted by the simulation are outlined in Figure 89.

The correspondence of differences between the 15 and 55Hz time slices (Figure 89) demonstrates the association of high porosity zones mapped during the initial portions of the study 
with high amplitudes in the low frequency end of the spectrum. As noted above, these results are interpreted to support the influence of frequency attenuation.

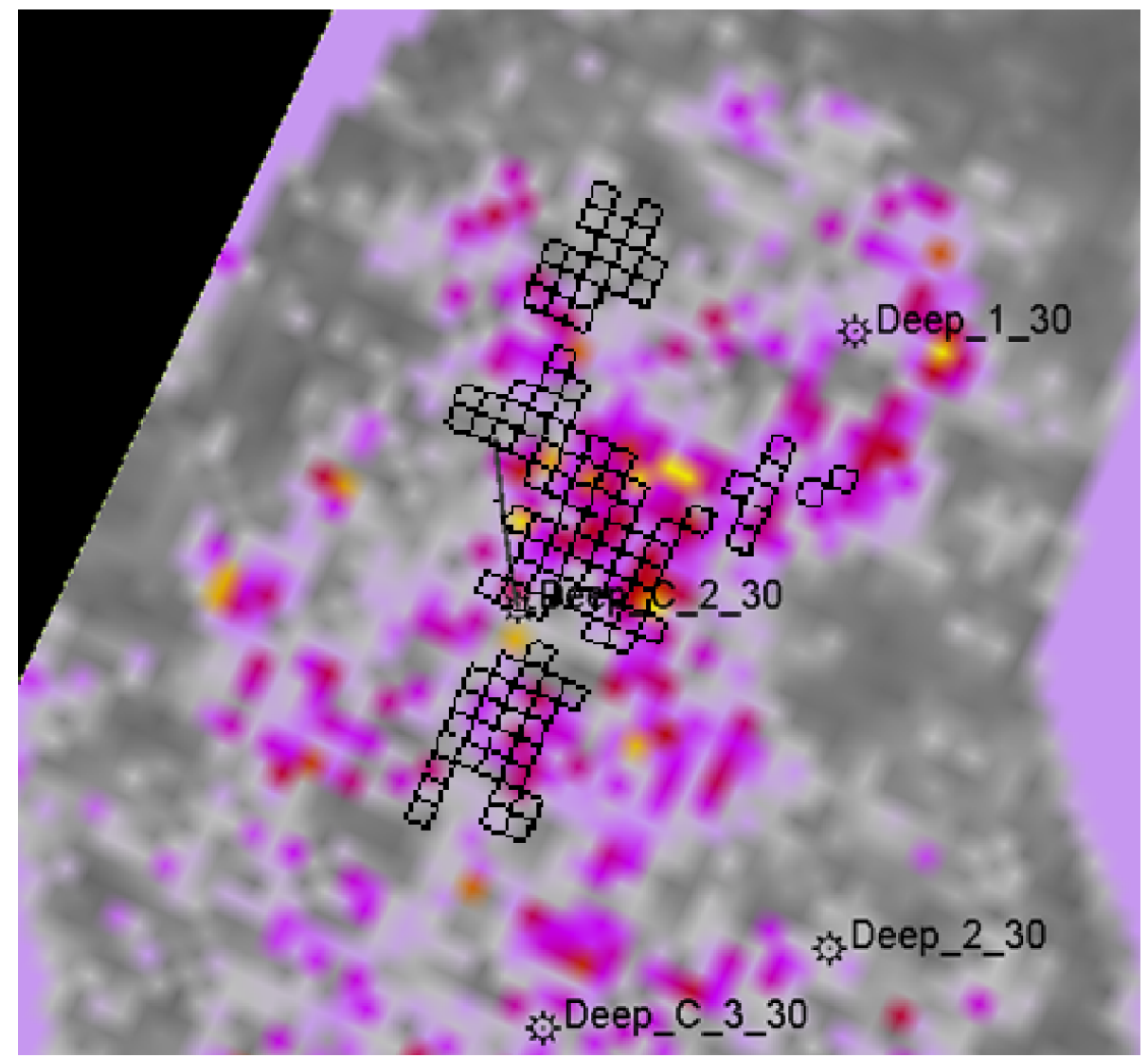

Figure 89: Iso-frequency Sum Difference Volume for $15 \mathrm{~Hz}$ minus $55 \mathrm{~Hz}$ at time slice $15 \mathrm{~ms}$ in the northern portion of the Charlton 30/31 oil field subtracted from the Iso-frequency Sum Difference Volume for $55 \mathrm{~Hz}$ (see Figure 88) overlaying with the cells predicted to be $\mathrm{CO}_{2}$ filled by the forward-looking reservoir simulation.

\subsection{Anisotropic Frequency Attenuation}

As previously stated the primary objective of this research is to develop a methodology that can be used to identify and characterize regions of drained, high fluid flow within a reservoir through the mapping of frequency attenuation, which may be either isotropic or anisotropic in nature. As shown in the previous section, interpretation of the Iso-frequency Sum Volumes, developed during the construction of the All Azimuth Fraction (AAF) Volumes, identified zones where strong low 
frequencies exist within the reservoir. These zones were identified during the preliminary investigations reported in Chapter 4 to correspond to zones of drained, high flow.

Additionally, the interpretation of azimuthal iso-frequency volumes was conducted in order to identify anisotropic frequency attenuation. The original volumes were used as no significant differences were observed between them and the normalized versions, (see Figure 82 and discussion in section 6.2 .1 of this chapter). Figure 90 shows the $15 \mathrm{~Hz}$ iso-frequency volume for azimuth $25^{\circ}$ (IF15A25) at $15 \mathrm{~ms}$ below the flatten A2 Carbonate. 


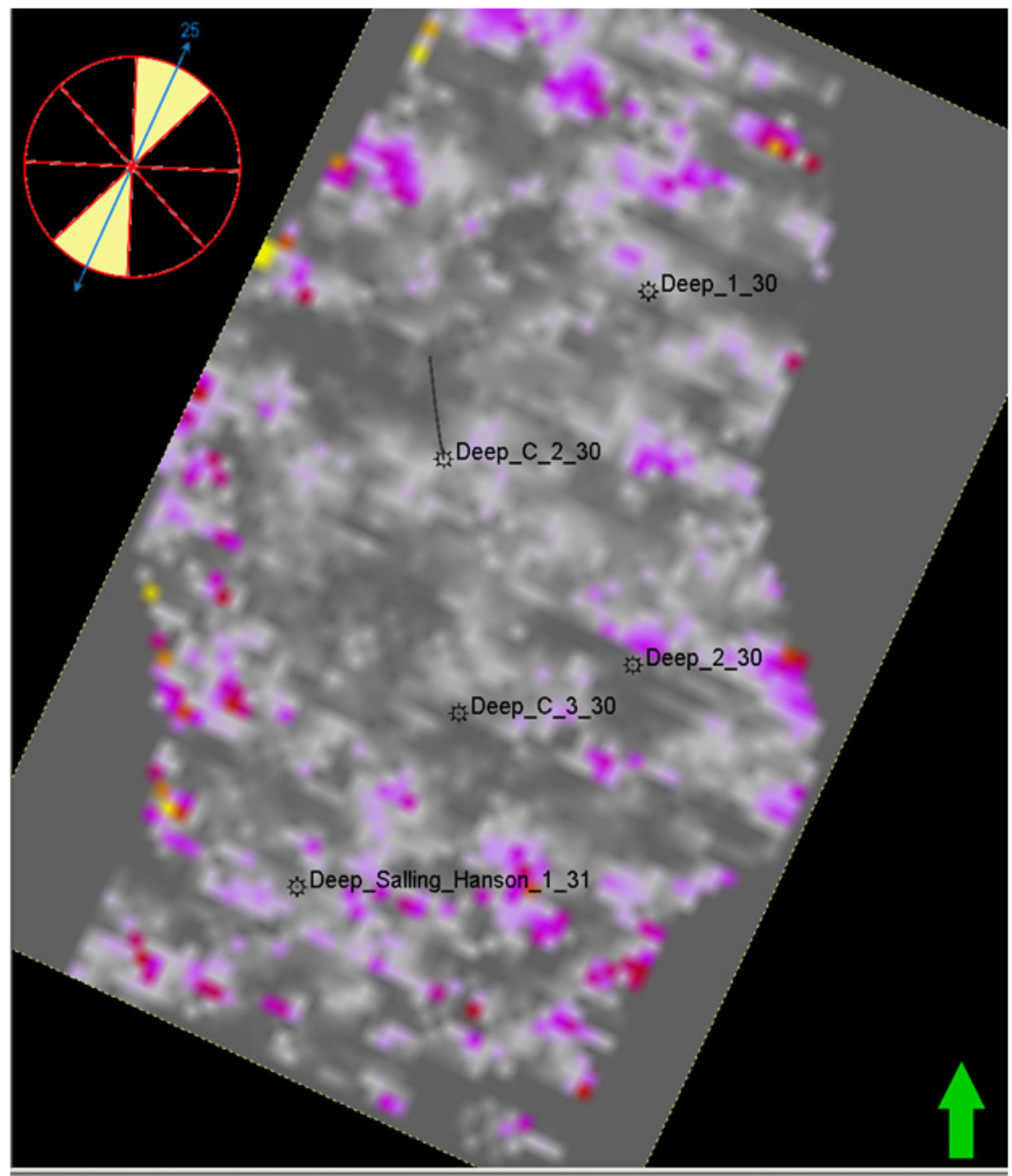

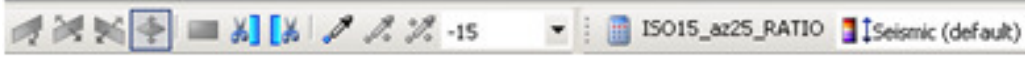

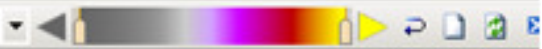

Figure 90: $15 \mathrm{~Hz}$ iso-frequency volume from Azimuth $25^{\circ}$ (IF15A25) at time slice $15 \mathrm{~ms}$. 
Figures 91, 92 and 93 show the $15 \mathrm{~Hz}$ Iso-frequency Volumes for the other azimuths at the same time slice, $15 \mathrm{~ms}$ below the top of the A2 Carbonate. An initial attempt was made to compare the amplitudes on the different azimuthal volumes.

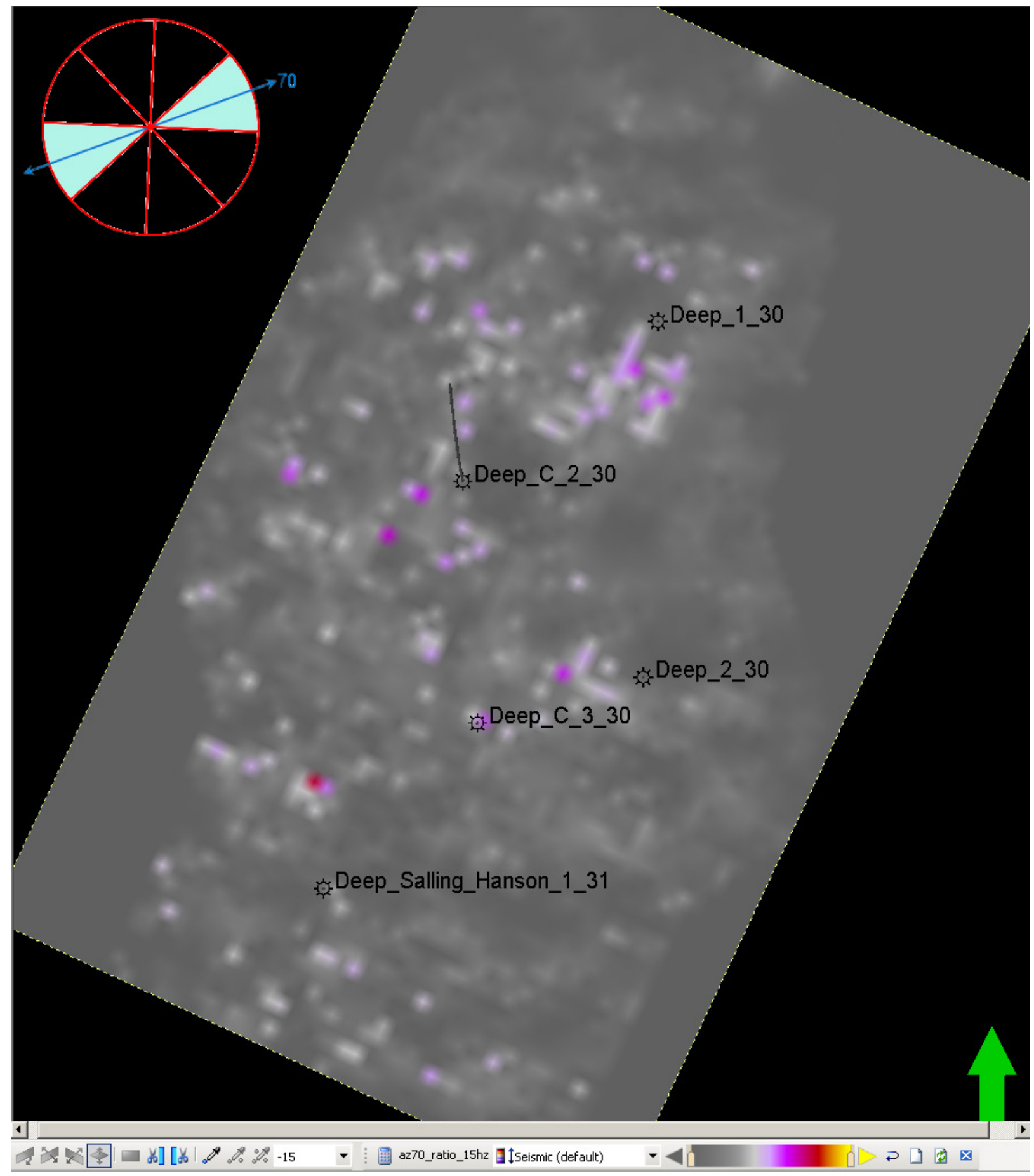

Figure 91: Iso-frequency $15 \mathrm{~Hz}$ ratio volume, azimuth $7^{\circ}$, (IF15A70) time slice $15 \mathrm{~ms}$. 
As can be seen in these time slices azimuth $25^{\circ}$ (Figure 90) shows the strongest amplitudes in $15 \mathrm{~Hz}$ whereas azimuth $70^{\circ}$ (Figure 91, above) shows the weakest amplitudes. Additionally, there are a number of areas or strong amplitudes in $15 \mathrm{~Hz}$ that are observed in common on multiple azimuths. This is true for the area immediately south of the EOR well, the State Charlton 1 - 30 well.

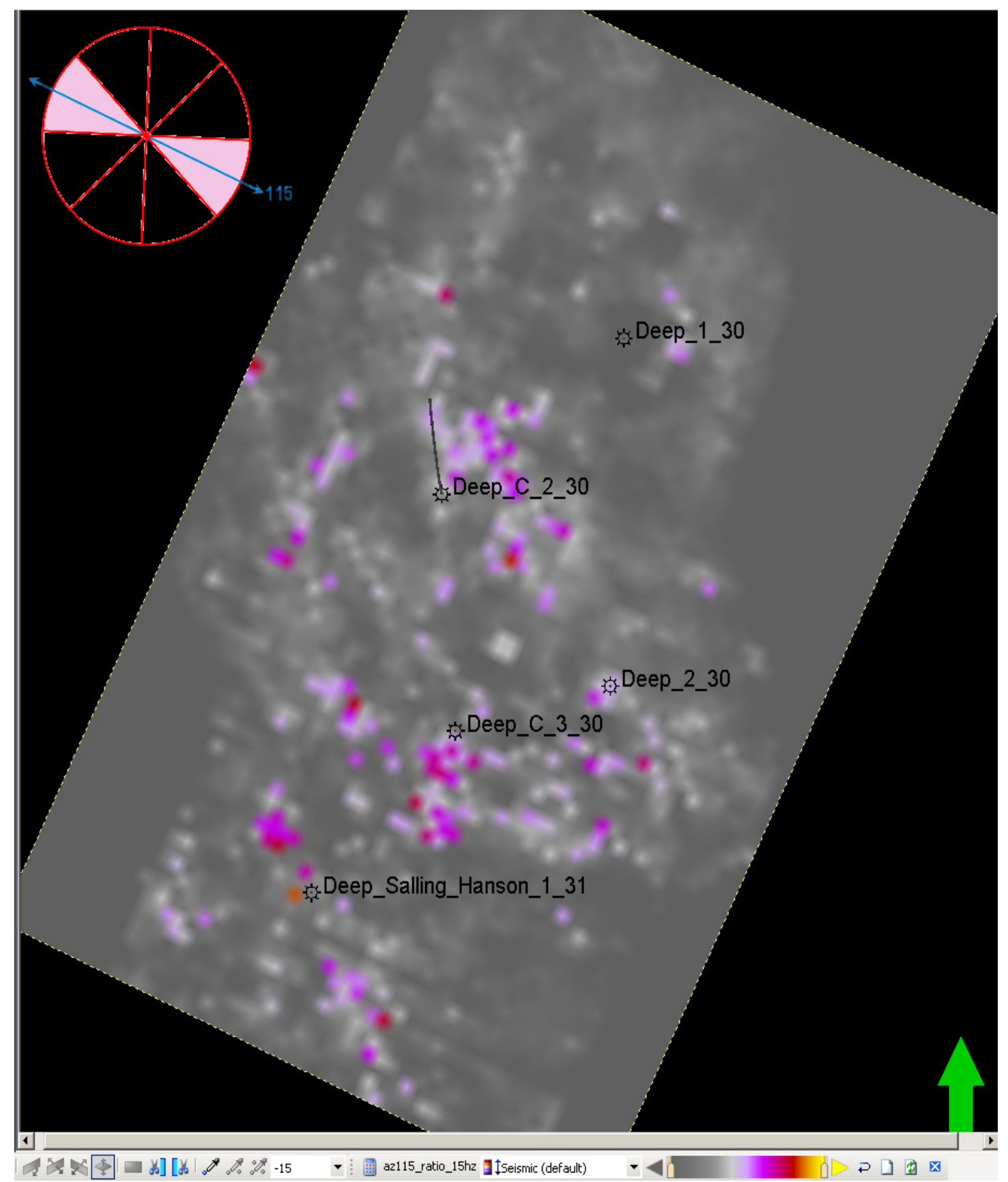

Figure 92: Iso-frequency $15 \mathrm{~Hz}$ ratio volume, azimuth $115^{\circ}$, (IF15A115) time slice $15 \mathrm{~ms}$. 
The area immediately south of the EOR well (the Deep_1_30 well) shows high amplitudes in $15 \mathrm{~Hz}$ images for the $25^{\circ}, 70^{\circ}$ and $160^{\circ}$ azimuths. Additionally, the area immediately to the east of the $\mathrm{CO}_{2}$ injection point, the $\mathrm{C} 2-30$ borehole, also shows high amplitudes on azimuth $25^{\circ}, 115^{\circ}$, and $160^{\circ}$. These areas would be interpreted as zones of isotropic frequency attenuation, even though they are not present on azimuth 115.

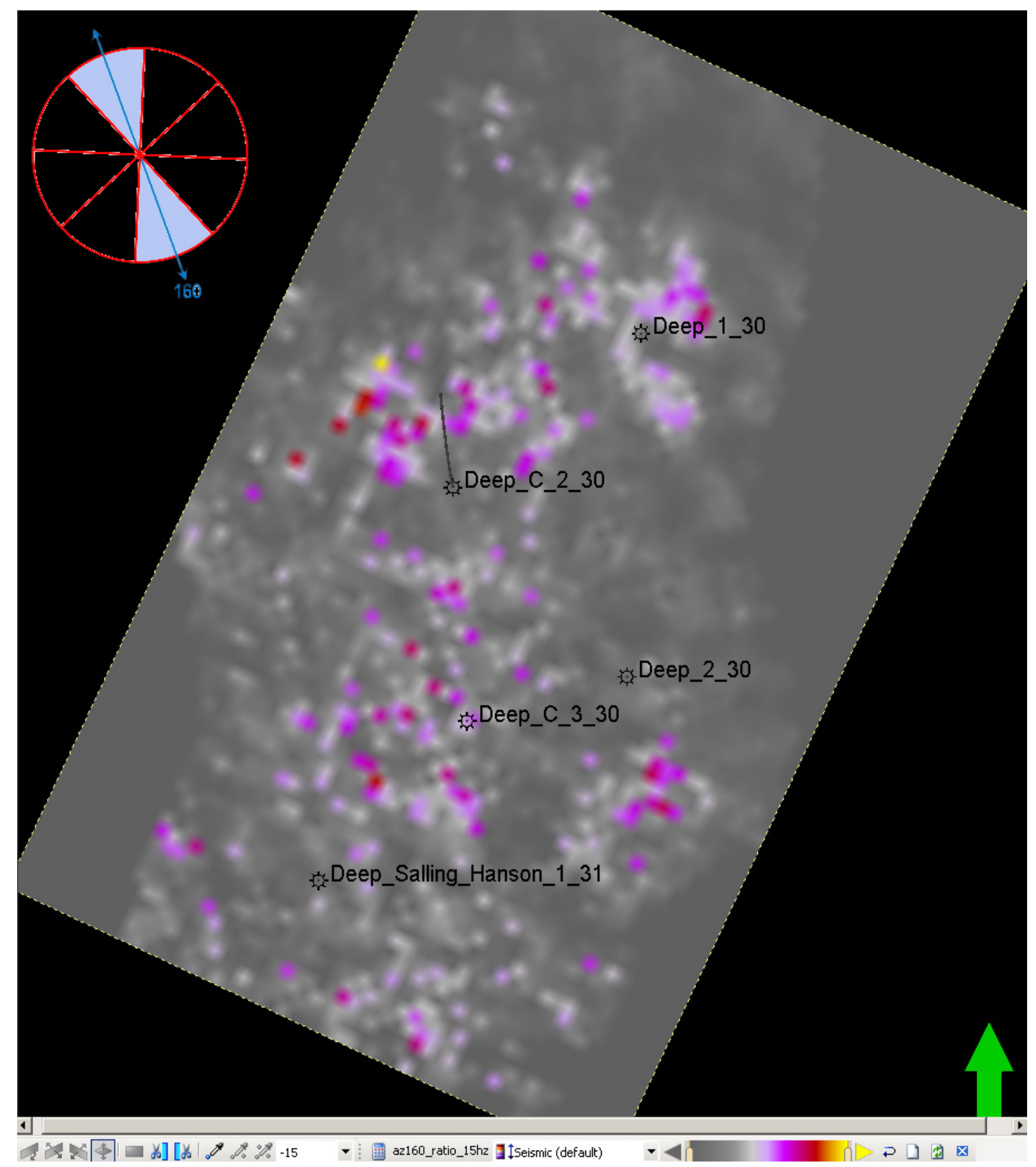

Figure 93: Iso-frequency $15 \mathrm{~Hz}$ ratio volume, azimuth $160^{\circ}$, (IF15A160) time slice $15 \mathrm{~ms}$. 
An open fracture system interpretation was developed within the reservoir by analyzing azimuthal volume pairs that were perpendicular to one another. The azimuthal seismic volume perpendicular to a fracture trend should display the greatest amount of frequency attenuation as a result of fracture compliance. Therefore, it was decided that azimuthal iso-frequency volumes would be interpreted in 2 sets of pairs, both $90^{\circ}$ to one another: azimuths $25^{\circ}$ with $115^{\circ}$ and azimuths $70^{\circ}$ and $160^{\circ}$.

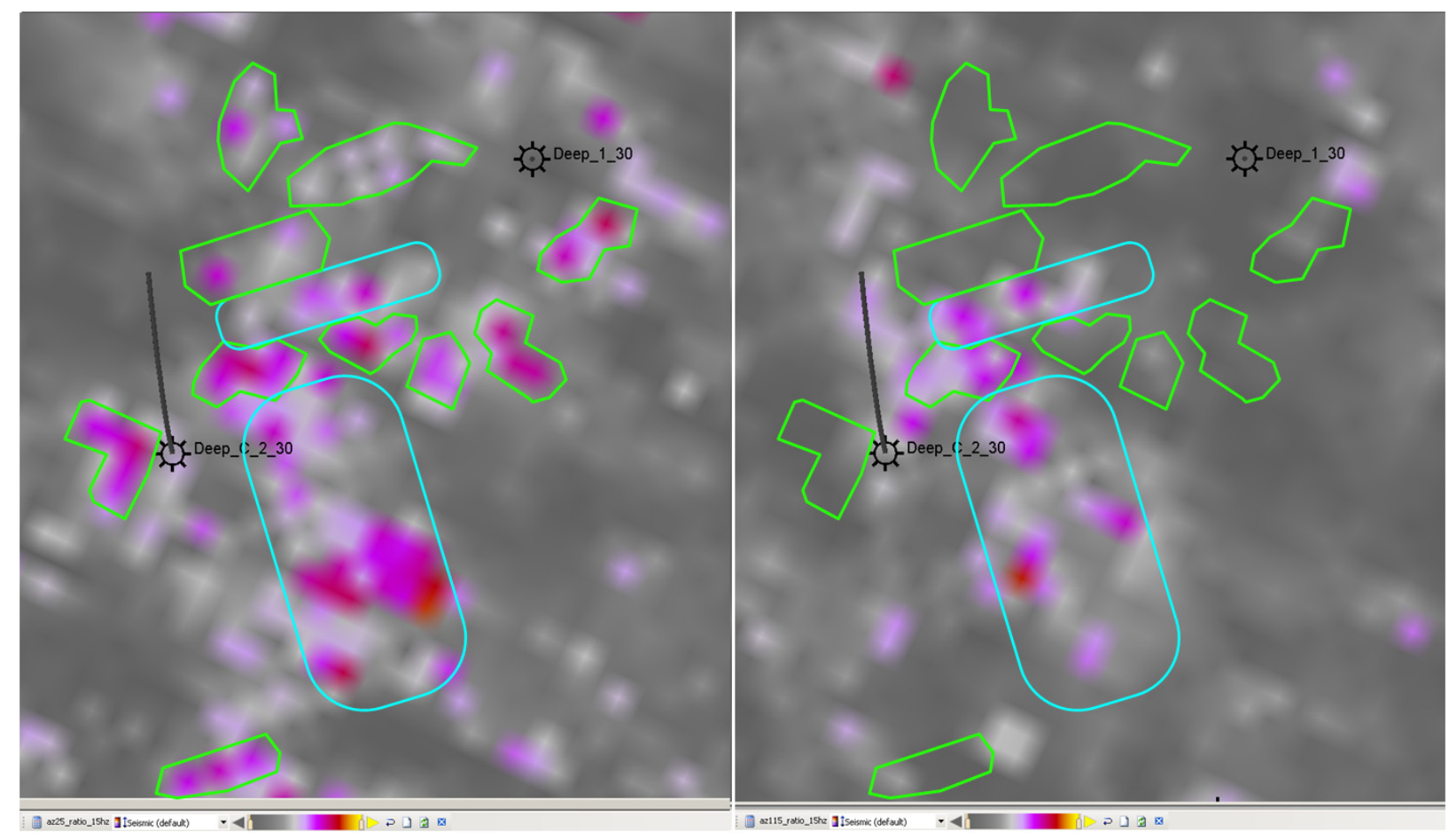

Figure 94: Azimuth $25^{\circ}$, iso-frequency $15 \mathrm{~Hz}$, time slice $15 \mathrm{~ms}$ (left) and Azimuth $115^{\circ}$, isofrequency $15 \mathrm{~Hz}$, time slice $15 \mathrm{~ms}$ (right). See text for annotation explanation.

Since no direct evidence of fracturing was obtained in the Charlton 30/31 field this portion of the study concentrated on the area between the $\mathrm{CO}_{2}$ injection point and the enhanced oil recovery well where anomalous amplitudes were observed on the 4D monitor survey (see Figure 80). Screen captures of this area were taken of the $15 \mathrm{~Hz}$ iso-frequency azimuthal volumes and loaded into a PowerPoint presentation. Using PowerPoint's "blink" emphasis animation the azimuthally normalized $15 \mathrm{~Hz}$ iso-frequency time slices were blinked back and forth in order to perform a comparison. The results of this comparison are shown in Figure 94 for azimuths $25^{\circ}$ and $115^{\circ}$. 
Coincident zones of $15 \mathrm{~Hz}$ high amplitude common to both azimuths are outlined in light blue. Zones of high amplitude in $15 \mathrm{~Hz}$ response which are only observed on azimuth $25^{\circ}$ or are anomalously strong on azimuth $25^{\circ}$ are outlined in green. As can be seen in this figure a series of green outlined anomalies occur along a linear path which transects the C 2-30 borehole and continues on to the east.

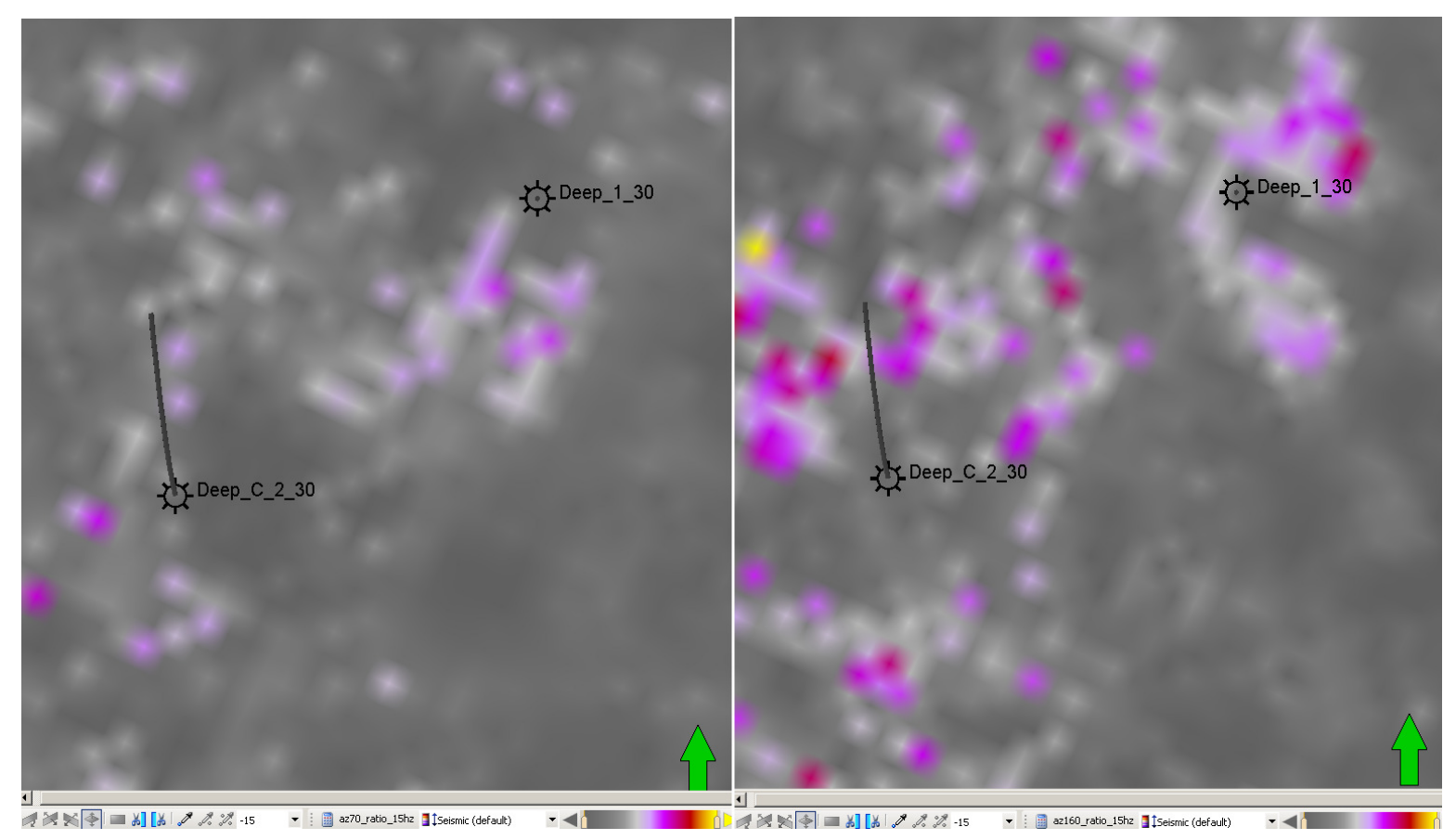

Figure 95: Azimuth $70^{\circ}$, iso-frequency $15 \mathrm{~Hz}$, time slice $15 \mathrm{~ms}$ (left) and Azimuth $160^{\circ}$, isofrequency $15 \mathrm{~Hz}$, time slice $15 \mathrm{~ms}$ (right).

Figure 95 shows the same comparison for azimuth $70^{\circ}$ and $160^{\circ}$. Azimuth $70^{\circ}$ shows a significant lack of strong amplitudes in $15 \mathrm{~Hz}$ throughout the area. Because of this almost no areas of high amplitude are found to be common between these two azimuths. Of note azimuth $160^{\circ}$ also shows high amplitudes immediately east of the $\mathrm{CO}_{2}$ injection point where none occur in azimuth $70^{\circ}$. Since these are 90 degrees apart this may be associated with a fracture trend.

\subsection{Conclusions}

The area immediately to the east and southeast of the injection site appears to have fractures as well as a fracture enhanced drained, high flow system within the reservoir's matrix. Areas that 
contain high amplitudes in $15 \mathrm{~Hz}$ with respect to the higher frequencies can be seen in common on multiple azimuths. These are areas of drained, high flow systems within the matrix and appear to correlate fairly well with the predictions made by the forward-looking reservoir simulation shown in Figure 89.

A linear trend of strong amplitude anomalies that are observed on $15 \mathrm{~Hz}$ iso-frequency volume for azimuth $25^{\circ}$ and, in some locations, azimuth $160^{\circ}$. This trend is not observed on the other two volumes which are perpendicular to these two and this trend is interpreted as an open fracture system which trends roughly east-west, outlined in green on Figure 94. As mentioned previously no evidence of open, natural fractures were observed in the well data. This is mainly due to the fact that image-based logging was not available until recently and not available when the wells in the Charlton 30/31 field were drilled and logged in the 1970's. Open fractures may exist in the field but they cannot be recognized with the available log set. The central azimuths for the development of the azimuthal volumes were thus selected based on the orientation of the survey in-line and cross-line directions. There is a low probability that any of the azimuths selected are exactly perpendicular to the trends of any fracture systems. Neither of these two azimuthal volumes $\left(25^{\circ}\right.$ and $\left.160^{\circ}\right)$ appear to be optimally aligned in order to strike this trend exactly perpendicularly. Of these two azimuths the anisotropy observed on azimuth $160^{\circ}$ appears greater than on azimuth $25^{\circ}$.

The results summarized above indicate that with the interpretation of azimuthal iso-frequency volumes zones of drained, high flow systems within the matrix can be distinguished from linear, drained, high flow zones related to fractures. This was accomplished through the comparison of the data volumes between azimuths that were perpendicular to one another. As a result of this analysis it was concluded that zones of isotropic and anisotropic frequency attenuation were observed. Fracture zones are interpreted based on differences in response observed on orthogonal azimuthal views. The observations presented in this chapter reveal that zones of isotropic and anisotropic frequency attenuation can be detected and differentiated. 


\section{CHAPTER 7 - DRAINED, HIGH FLOW SYSTEM CHARACTERIZATION AND COMPARISON WITH 4D SEISMIC}

\subsection{Introduction}

In chapter 6 the interpretation of azimuthally normalized iso-frequency volumes was investigated in order to determine if drained, high flow zones in the matrix could be distinguished from drained, high flow systems related to fractures. This was accomplished through the comparison of the data volumes between azimuths that were perpendicular to one another. As a result of this analysis it was concluded that zones of isotropic and anisotropic frequency attenuation were observed.

In this chapter, analysis is undertaken to determine if the zones of isotropic and anisotropic frequency attenuation are associated with changes of amplitude observed in the final migrated stack data compiled from the Baseline and Monitor surveys (see Chapter 4). As reported previously in Chapter 4, evidence from the analysis of a number of different data sets aligned with zones of increased amplitude observed on the 4D Monitor survey. These zones of increased amplitude are believed associated with increased reservoir pressure due to the replacement of oil by the injected $\mathrm{CO}_{2}$.

\subsection{D Survey Comparative Analysis}

Figures 63, 64, 65, 66, 67, 68 and 71 (Chapter 4) show the features in the study area interpreted as high porosity $\mathrm{CO}_{2}$ saturated areas within the reservoir observed in the Monitor survey. Figure 72 shows the comparison with between the iso-frequency normalized $15 \mathrm{~Hz}-55 \mathrm{~Hz}$ difference volume from the Baseline Azimuthal surveys and the same time slice taken from the for the Monitor survey. As noted previously this time slice shows the high amplitude zone extends from the $\mathrm{CO}_{2}$ injection point towards the EOR producer. In chapter 4 these high amplitude features were shown to be anomalous with the predicted locations of the injected $\mathrm{CO}_{2}$ made by the forward-looking reservoir simulation. 


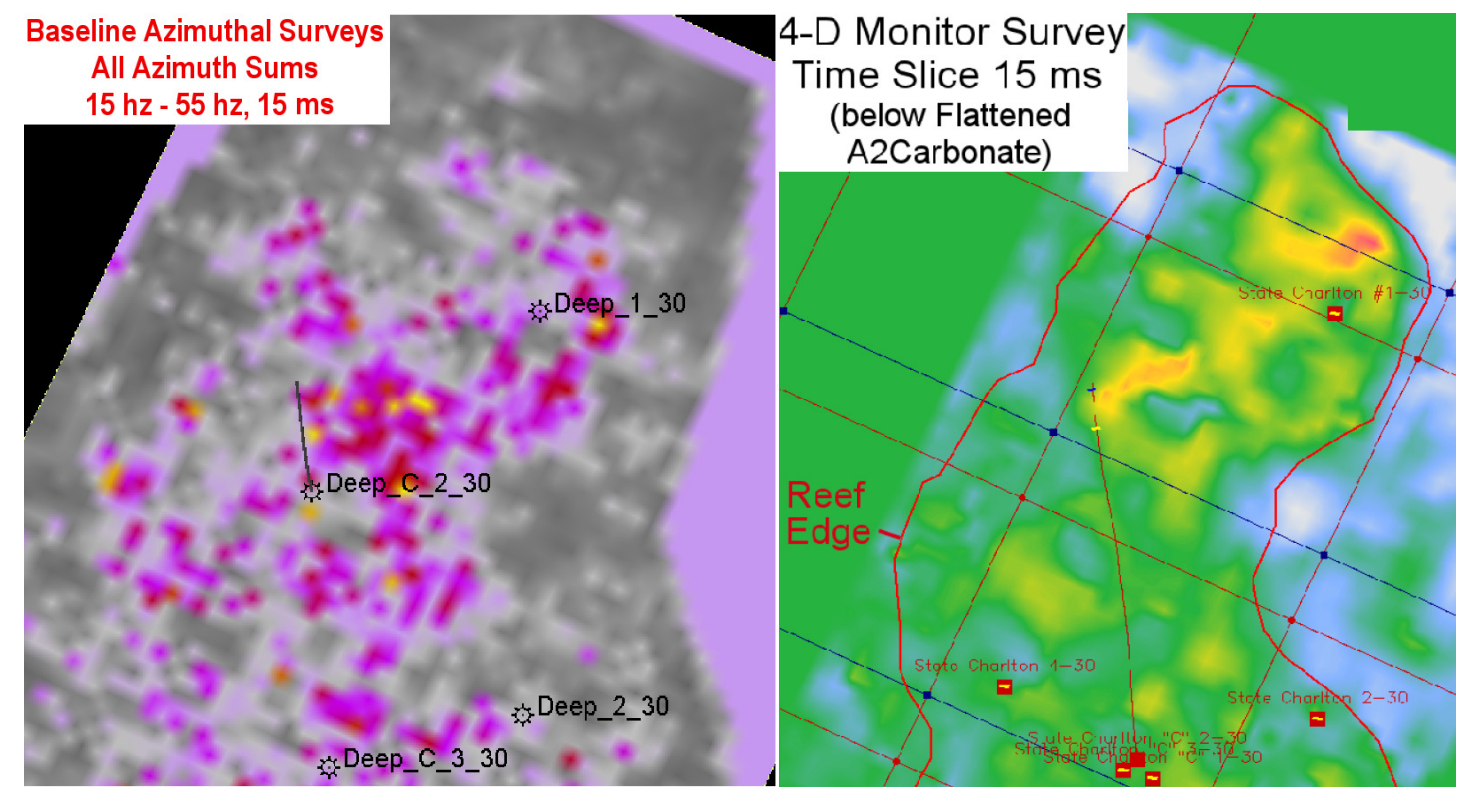

Figure 96: Comparison between the $15 \mathrm{~ms}$ time slice from the Iso-Frequency Normalized 15 $\mathrm{hz}$ - $55 \mathrm{hz}$ Difference Volume of the Baseline Azimuthal Surveys and the $15 \mathrm{~ms}$ time slice from the flattened 4D Monitor Survey Amplitude Volume.

Figure 96 shows a significant amount of frequency attenuation has occurred at the same location where this high amplitude zone is observed on the full azimuth for the 4D Monitor survey.

\subsection{Iso-frequency Normalized Comparative Analysis}

To compare the various azimuths for a specific iso-frequency I normalized the response for a given azimuth using a ratio of the response for a given azimuth to the sum of responses for all azimuths at a given frequency (see discussion of All Azimuth Fraction volumes in 5.4.1 and Table 3 in Chapter 5). This process yields four azimuthal $15 \mathrm{~Hz}$ All Azimuth Fraction volumes (AAF15A25, AAF15A70, AAF15A115 and AAF15A160) in which amplitudes represent a percentage (or fraction) of the total response from all azimuths. These were used to characterize high-amplitude features as high porosity zones with isotropic or anisotropic characteristics. Zones that are anisotropic are interpreted to be associated with a fracture zone. The results of this comparison are shown in the following figure for $15 \mathrm{~ms}$ below the top of A2 Carbonate. 


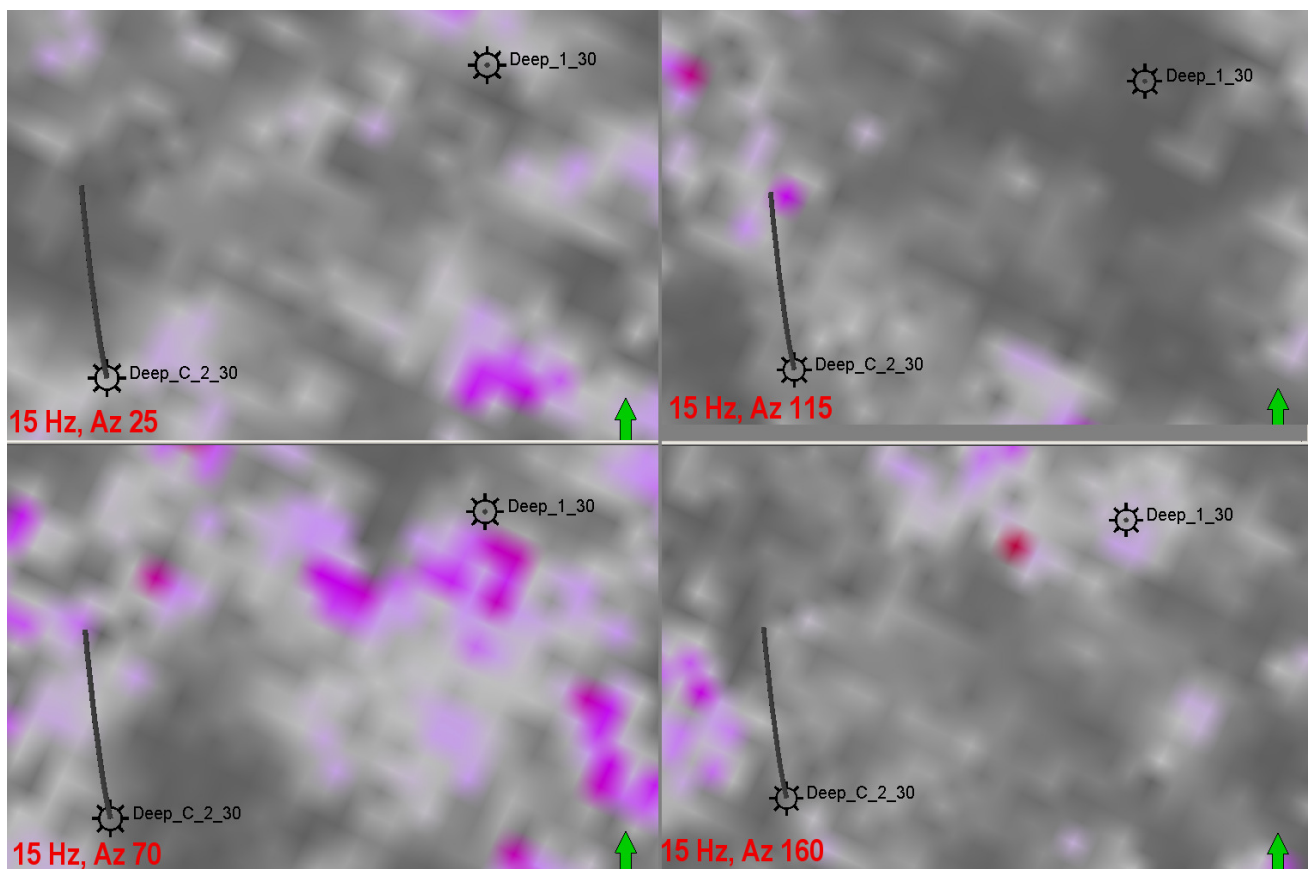

Figure 97: Time slice $15 \mathrm{~ms}$ below the top of the A2 Carbonates from the $15 \mathrm{~Hz}$ iso-frequency normalized volume.

As can be seen on Figure 97 the percent change in amplitude between orthogonal pairs (the $25^{\circ}$ and $115^{\circ}$ azimuths and the $70^{\circ}$ and $160^{\circ}$ azimuths) is significant only between the $70^{\circ}$ and $160^{\circ}$ azimuths. The area between the tip of the injection lateral and the producing well observed along azimuth 2 (central azimuth of $\mathrm{N} 70^{\circ} \mathrm{E}$ ) has distinctively higher amplitude than present in the orthogonal, azimuth 4 (central azimuth of $\mathrm{N} 160^{\circ} \mathrm{E}$ ).

Figure 98 shows a comparison of the All Azimuth Fraction (or frequency normalized) $15 \mathrm{~Hz}$ volume at time slice $15 \mathrm{~ms}$ for azimuth 2 (central azimuth of $\mathrm{N} 70^{\circ} \mathrm{E}$ ) compiled from the monitor survey. The figure focuses on the area between the $\mathrm{CO}_{2}$ injection point and the EOR producer. Zones of increased amplitude strength at $15 \mathrm{~Hz}$ on azimuth 2 (central azimuth of $\mathrm{N} 70^{\circ} \mathrm{E}$ ) appear to relate to two zones of increased amplitude observed on the full azimuth Monitor survey. These two zones are outlined in blue and are labeled 1 and 2 in Figure 98. In accordance with my theory I would interpret these areas as zones of $\sim \mathrm{N} 20^{\circ} \mathrm{W}\left(\sim \mathrm{S} 20^{\circ} \mathrm{E}\right)$ trending open fractures. The amplitude increase observed in the low frequencies would be interpreted as a result of attenuation of high frequencies in the 
direction orthogonal to this trend if the All Azimuth Fraction (or frequency normalized) volumes are sensitive to this.

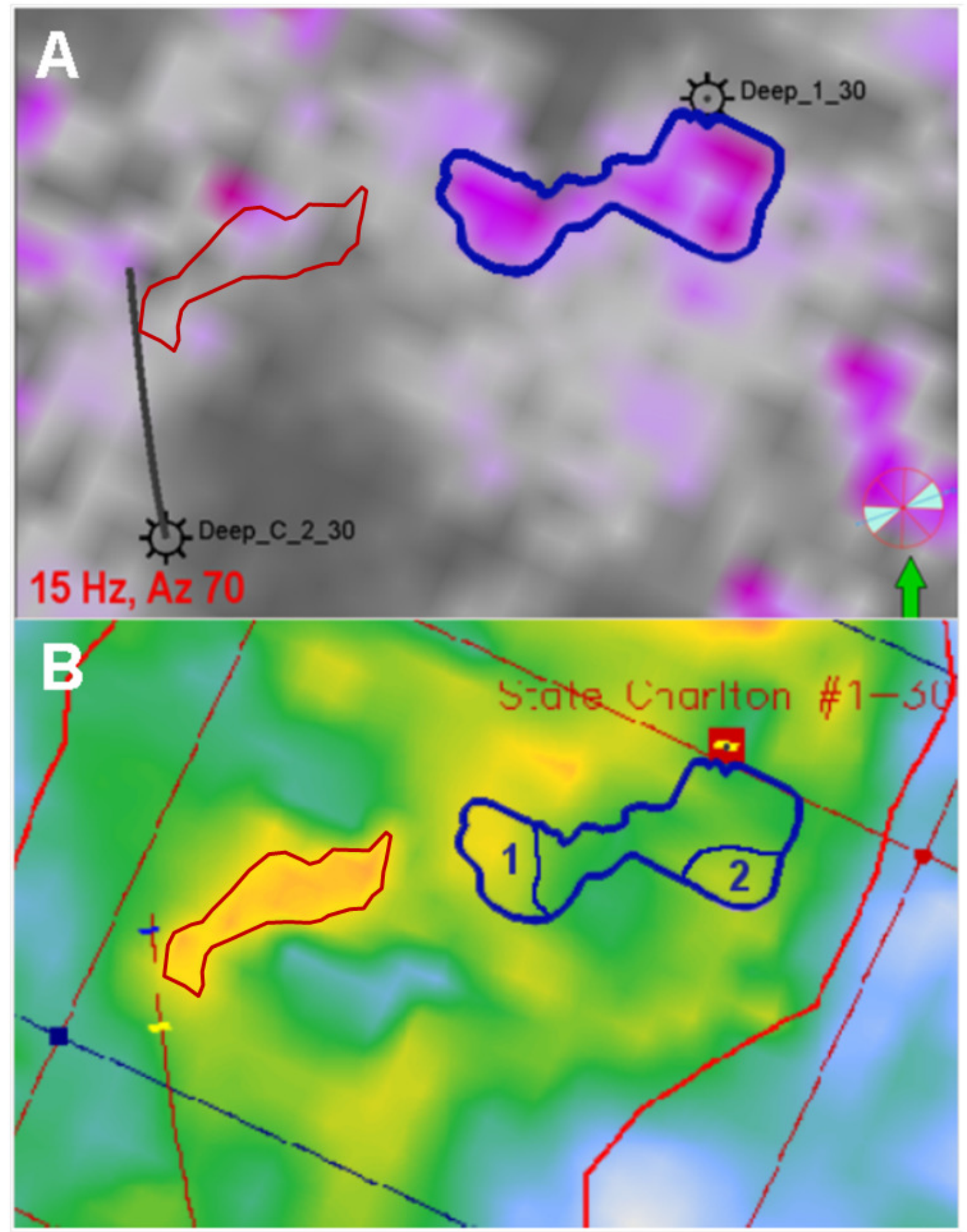

Figure 98: Comparison between iso-frequency normalized $15 \mathrm{~Hz}$ volume for azimuth $70^{\circ}$ (A) and monitor survey amplitude volume (B). 
However, while AAF15A70 (azimuth 2, central azimuth of $\mathrm{N} 70^{\circ} \mathrm{E}$ ) displays the greatest amplitude difference no alignment is seen with the amplitude anomaly observed in the Monitor survey. Figure 98 shows this comparison. Two other All Azimuth Fraction volumes, AAF15A25, (azimuth 1, central azimuth $25^{\circ}$ ) and AAF15A160, (azimuth 4, central azimuth $160^{\circ}$ ), display lineaments at a significant location within the survey. Figure 99 shows the $15 \mathrm{~ms}$ time slice from the AAF15A25 and AAF15A160. These time slices are shown side-by-side with no interpretation on the left and the same displays but with interpretation shown on the right. Subtle changes can be observed in the vicinity of the $\mathrm{CO}_{2}$ injection point trending east northeastward towards enhanced oil recovery producer. Although the linear trends indicated do not show significantly high amplitudes their location is quite significant when compared to the features observed on Figure 89 in chapter 6, which shows the non-normalized or raw amplitudes for iso-frequency $15 \mathrm{~Hz}$, azimuth $25^{\circ}$ for time slice 15 ms, (IF15A25). These features could be interpreted as additional evidence of linear fracture trends at this location.

This east northeast linear feature, which extends from the bottom hole location for the State Charlton "C" 2-30 well towards the EOR producer, however it does not occur at the same location where a set of increased amplitudes are observed on the $15 \mathrm{~Hz}$ iso-frequency volume for azimuth $25^{\circ}$, Figure 90 or where the amplitude anomaly is observed in the Monitor survey, which is shown in Figure 99, A2 and B2, as a red outline. The amplitude change at this frequency for azimuths separated by $90^{\circ}$ (azimuth $25^{\circ}$ and $115^{\circ}$ as well as azimuths $70^{\circ}$ and $160^{\circ}$ ), see Figure 97 and 99 , suggests that this may be a linear trend of open natural fractures that are creating anisotropy at this location within the reservoir. However, the lack of increased amplitude in the $15 \mathrm{~Hz}$ frequency in this area as well as the poor alignment with the amplitude anomaly from the Monitor survey indicate that the All Azimuth Fraction volumes (or the frequency normalized volumes) are not good indicators of frequency attenuation. 


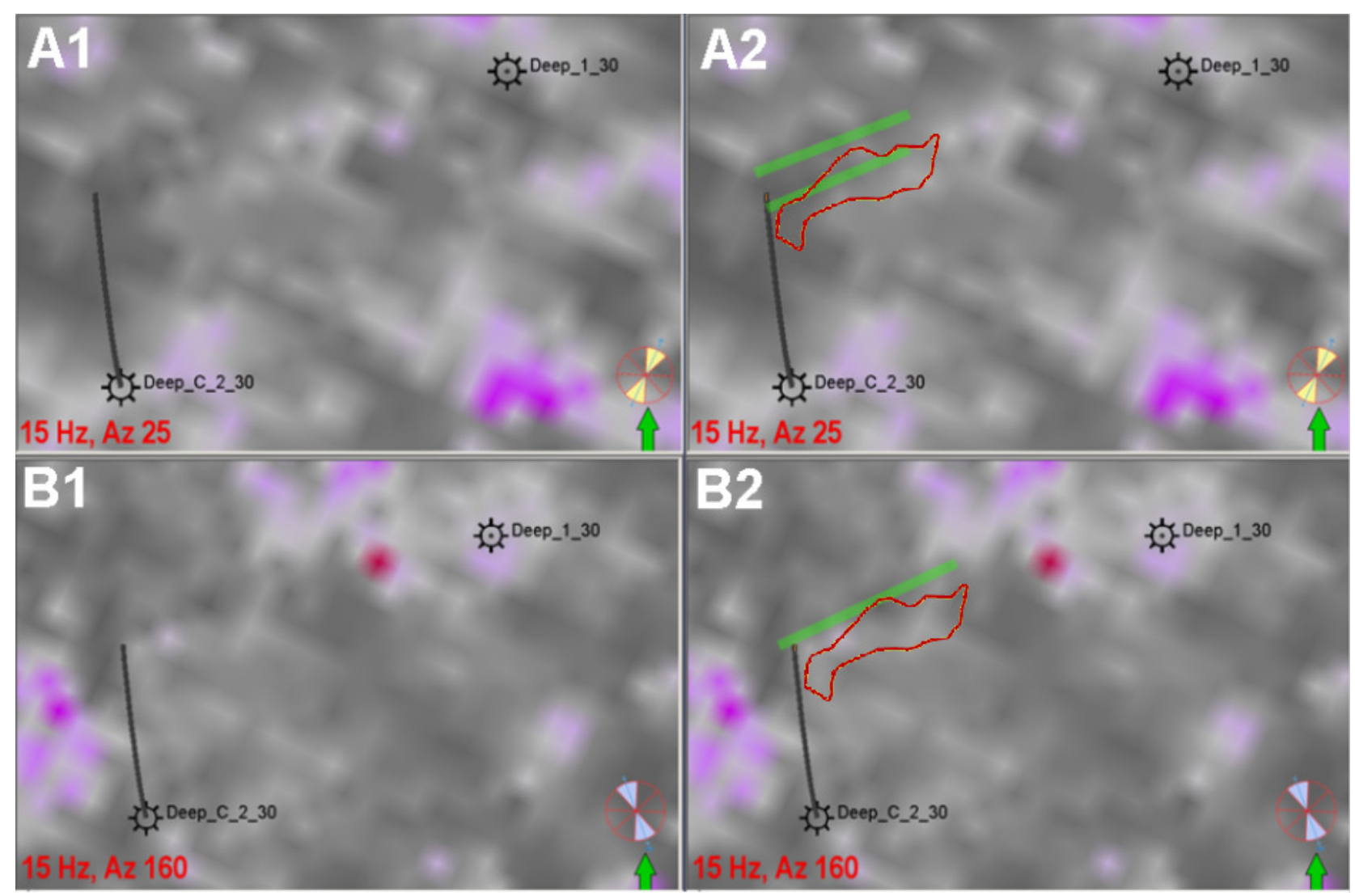

Figure 99: $15 \mathrm{~ms}$ time slice from the normalized $15 \mathrm{~Hz}$ iso-frequency volume. The time slice was extracted from the $25^{\circ}$ azimuth $(\mathrm{A})$ and $160^{\circ}(\mathrm{B})$. Figures $\mathrm{A} 2$ and $\mathrm{B} 2$ show interpreted features (green) extending NE from the end of the State Charlton "C" 2-30 lateral.

\subsection{Reservoir Characterization Models}

Multiple attempts were made to characterize the drained, high flow system distribution within the Charlton 30/31 oil field using the azimuthal iso-frequency seismic volumes. The original iso-frequency volumes developed from the azimuthal volumes were examined along with the Selected Frequency Fraction volumes and the All Azimuth Fraction volumes. While the original azimuthal iso-frequency volumes were quite similar to the Selected Frequency Fraction volumes the All Azimuthal Fraction volumes proved to be quite different. Additionally, observations made with the original azimuthal iso-frequency volumes and the Selected Frequency Fraction volumes indicated a good alignment with other porosity indicators discussed in previous chapters. However, little to no alignment was seen with the All Azimuth Fraction volumes. 
The primary goal of this study has been to develop a reservoir characterization methodology that would not only detect drained, high flow systems within the reservoir but also distinguish between those associated with the matrix and those related to open fracture systems. Towards this end I decided to perform the final reservoir characterization using the original azimuthal isofrequency volumes. This was accomplished by combining information from the two different pairs of orthogonal azimuths. For the first model Reservoir Characterization Model \#1, the original azimuthal iso-frequency $15 \mathrm{~Hz}$ iso-frequency volumes for IF15A25, (azimuth 1, central azimuth $25^{\circ}$ ) and IF15 A115 (azimuth 3, central azimuth $115^{\circ}$ ) were examined to identify the drained, high flow systems associated with the reservoir's matrix. Additionally, zones of increased reflection strength that were only observed on one azimuth and not the other were identified. Figure 100 shows this comparison.

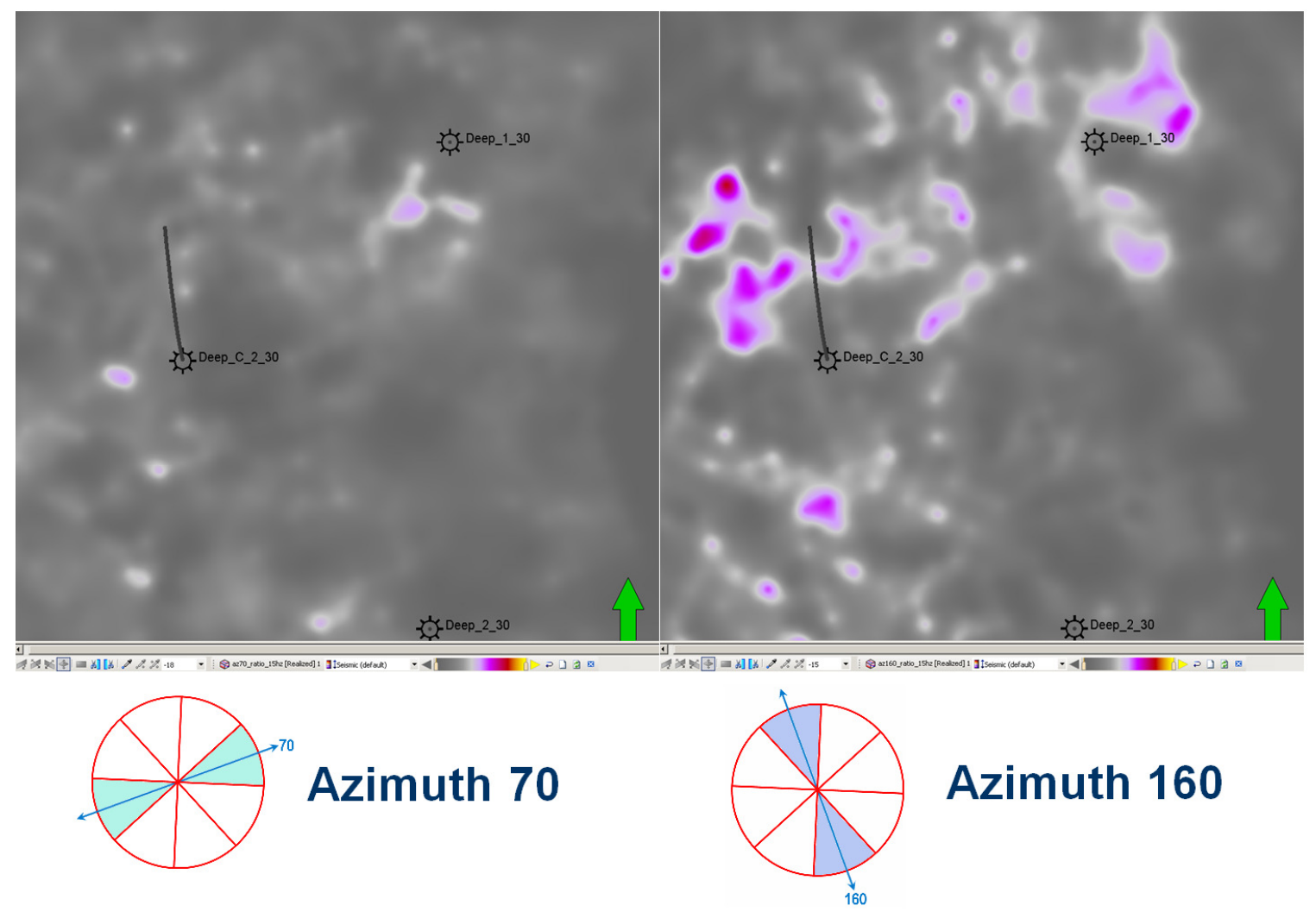

Figure 100: Comparison of IF15A70, iso-frequency $15 \mathrm{~Hz}$, azimuth $70^{\circ}$ for time slice $15 \mathrm{~ms}$ (left) and IF15A160, iso-frequency $15 \mathrm{~Hz}$, azimuth $160^{\circ}$ for time slice $15 \mathrm{~ms}$ (right). 
Additionally, an examination of the azimuthal $15 \mathrm{~Hz}$ iso-frequency volumes revealed that on azimuth 160 at the $15 \mathrm{~ms}$ time slice there exists an east northeast trending linear feature. These areas of increased amplitudes originate at the $\mathrm{CO}_{2}$ injection point in the State Charlton "C" 2-30 borehole and trending towards the EOR producer, (see Figure 100, on right). This increased reflection strength is not observed on the orthogonal $70^{\circ}$ azimuth. Following the theory put forth in this study, the differences of amplitude observed on orthogonal azimuths suggest the presence of an open natural fracture trends. Figure 101 below shows the interpretation of the location and orientation of these features. The area between the injector and EOR well on IF15A160, iso-frequency $15 \mathrm{~Hz}$, azimuth 4 (central azimuth $160^{\circ}$ ) is shown on the left (A) for the time slice $15 \mathrm{~ms}$ and the interpreted fracture locations and directions are show on the right (B). Fracture locations observed on IF15A160 (azimuth $160^{\circ}, 15 \mathrm{~Hz}$ ) at $15 \mathrm{~ms}$ time slice are indicated in purplish, irregular areas, the shapes of which are taken directly from the shapes of the high reflection strength areas. Fracture orientations are indicated by the black lines within those areas (which are oriented $\mathrm{N} 50^{\circ} \mathrm{E}$ ) and are consistent those predicted by this study's theory with regard to frequency attenuation caused by fracture compliance for azimuth $160^{\circ}$. The few zones observed on IF15A70 (iso-frequency $15 \mathrm{~Hz}$, azimuth 2 , central azimuth $70^{\circ}$ ), on $15 \mathrm{~ms}$ time slice that are unique to that azimuth are indicated in yellow. The interpretation of these two, perpendicular azimuths produced one interpretation of potential fracture trends at the top of the reef and is designated Fracture Characterization \#1 


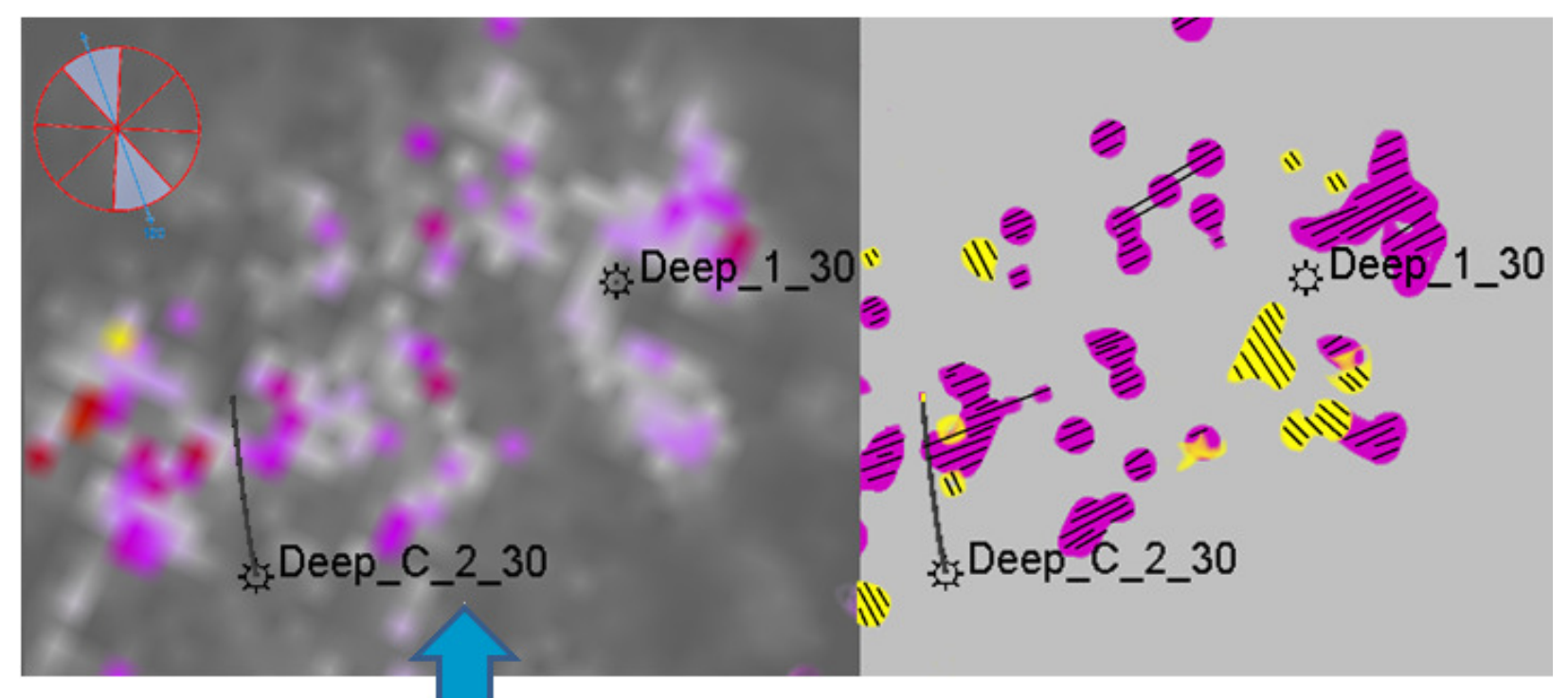

Azimuth $160^{\circ}$

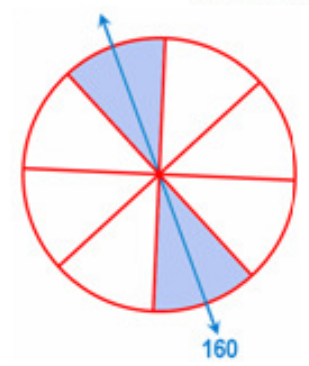

Azimuth $70^{\circ}$

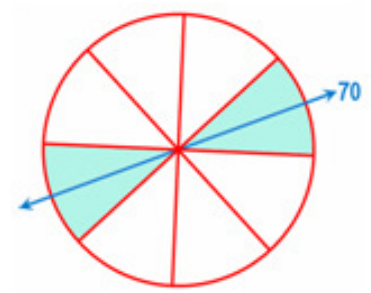

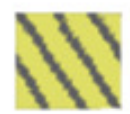

Figure 101: Fracture Characterization Model \#1 (on right) developed by comparing features observed on IF15A160, (15 Hz iso-frequency, azimuth 160 volume) at time slice $15 \mathrm{~ms}$ (shown at left) with azimuthally normalized $15 \mathrm{~Hz}$ iso-frequency, azimuth 70 volume. See text for explanation.

This same analysis was performed for IF15A25, iso-frequency $15 \mathrm{~Hz}$, azimuth 1, (central azimuth $25^{\circ}$ ) and IF15A115, iso-frequency $15 \mathrm{~Hz}$, azimuth 3, (central azimuth $115^{\circ}$ ) at the $15 \mathrm{~ms}$ time slice. The comparison of these two azimuths is shown in Figure 102 and the results of the interpretation are shown in Figure 103. Large areas of increased reflection strength on azimuth 25 (shown in green) can be seen east and southeast of the $\mathrm{CO}_{2}$ injection point. Additionally, a number of smaller areas with increased reflection strength are observed in various other locations. Only three areas of increased reflection strength on the azimuth 115 (shown in blue) are also observed. These areas of increased reflection strength are also interpreted as zones containing open, natural fractures, 
however, with different fracture orientations, as indicated by the black lines. This interpretation is designated Fracture Characterization \#2.

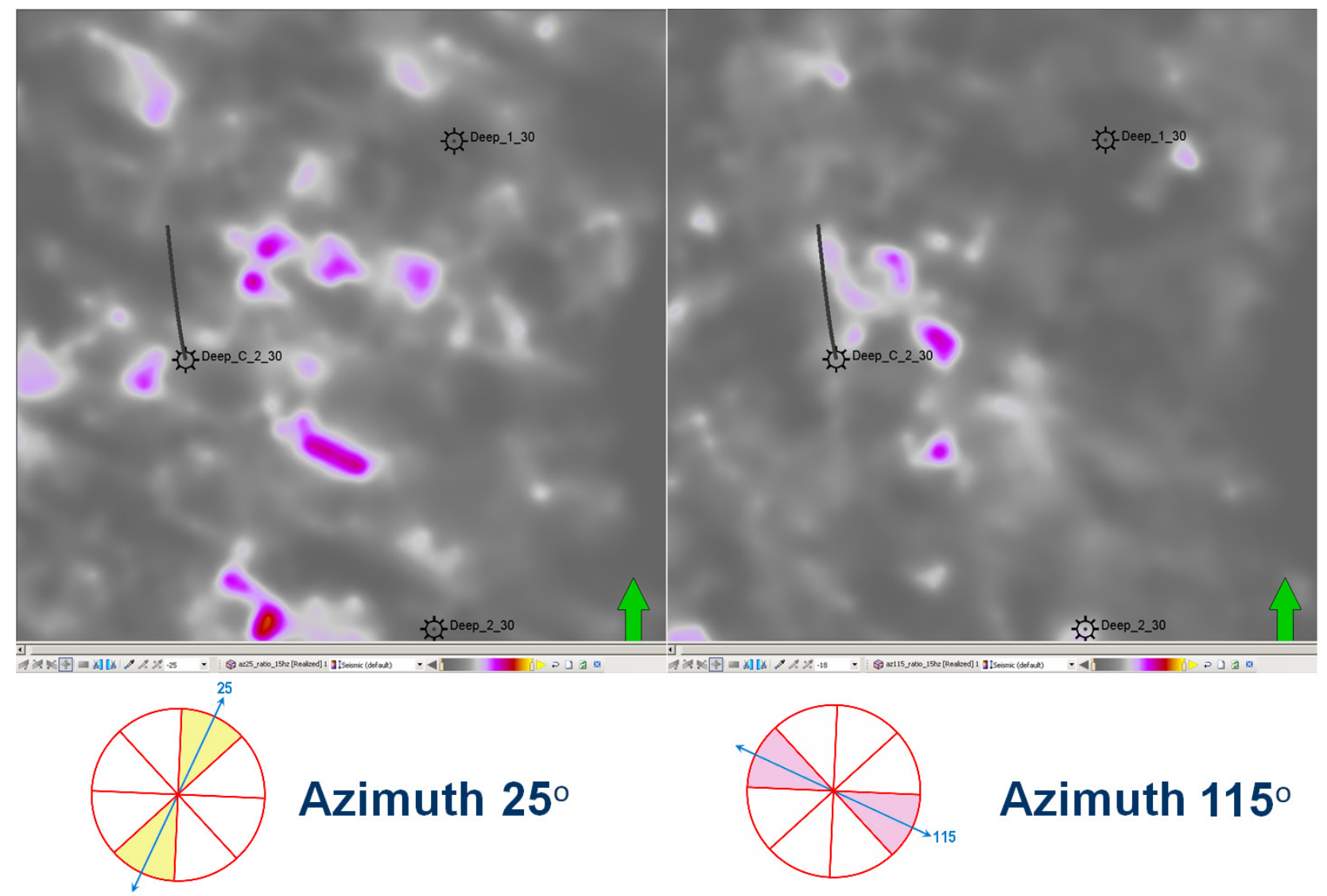

Figure 102: Azimuth $25^{\circ}$, iso-frequency $15 \mathrm{~Hz}$, time slice $15 \mathrm{~ms}$ (left) and Azimuth $\mathbf{1 1 5}^{\circ}$, isofrequency $15 \mathrm{~Hz}$, time slice $15 \mathrm{~ms}$ (right). See text for annotation explanation. 


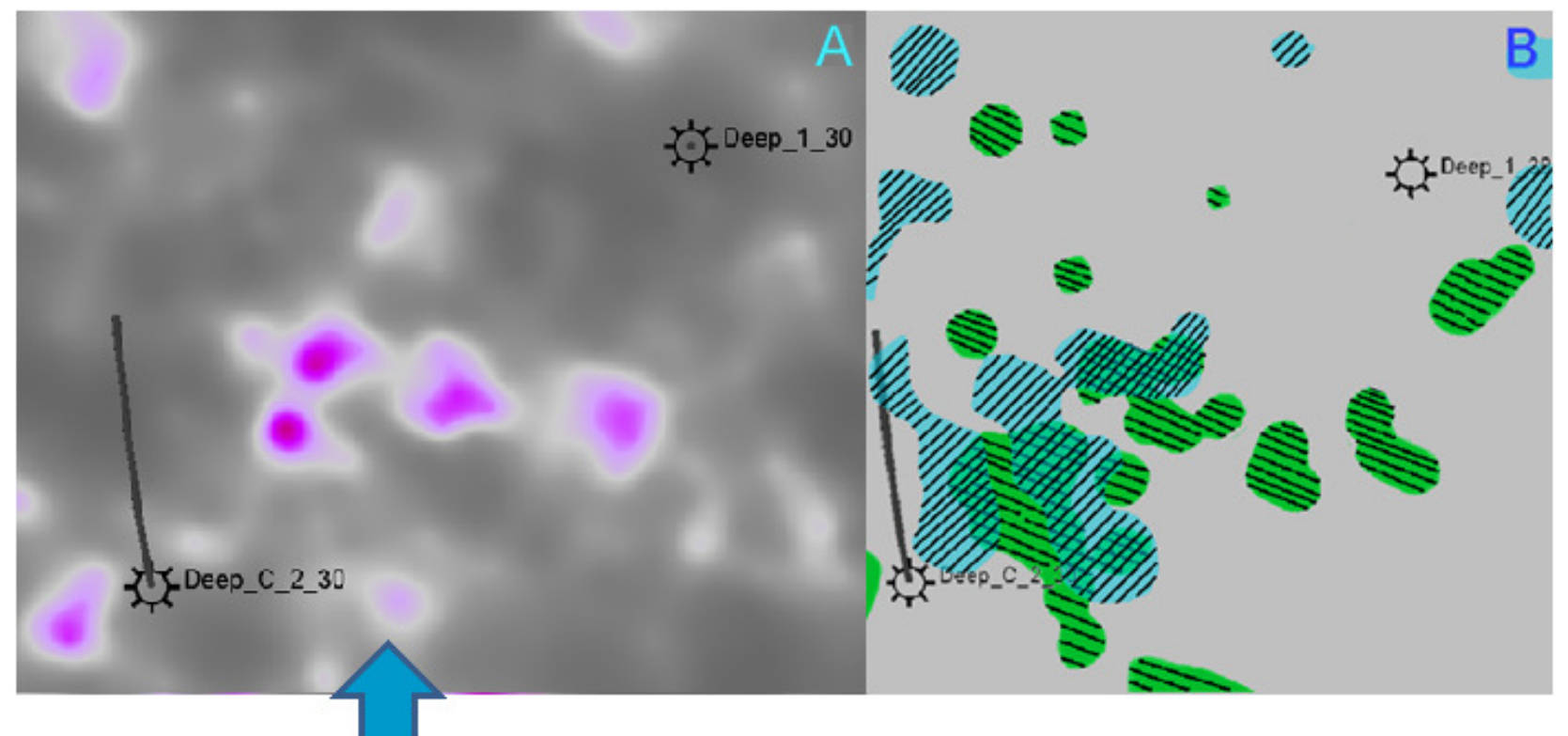

Azimuth $2^{\circ}$

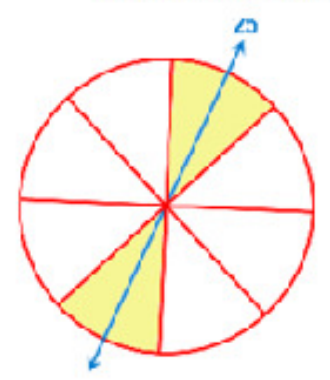

Azimuth $115^{\circ}$

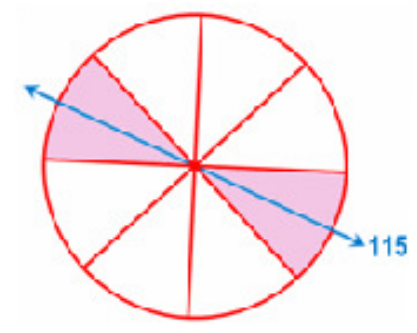

IIII

Figure 103: Fracture Characterization Model \#2 developed by comparing features observed on azimuthally normalized $15 \mathrm{~Hz}$ iso-frequency, azimuth 25 volume at time slice $15 \mathrm{~ms}$ with azimuthally normalized $15 \mathrm{~Hz}$ iso-frequency, azimuth 115 volume. See text for explanation.

Next Fracture Characterizations \#1 and \#2 were combined graphically in an attempt to identify zones at the top of the reservoir where increased reflection strength existed on more than one azimuth. This combined fracture model is shown in Figure 104. Zones of increased reflection strength that are in common on multiple azimuths are circled in red and are designated as zones of "overlap". These zones of overlap are generally smaller and fewer in number than the zones interpreted to be fracture related on the various azimuths. No discernable pattern to their distribution 
is observable concerning their locations or shapes. However, an examination of the combined fracture model does indicate that two fracture trends dominate, those observed on azimuths $25^{\circ}$ and $160^{\circ}$, while the other two fracture trends, those observed on azimuths $70^{\circ}$ and $115^{\circ}$, are mainly absent.

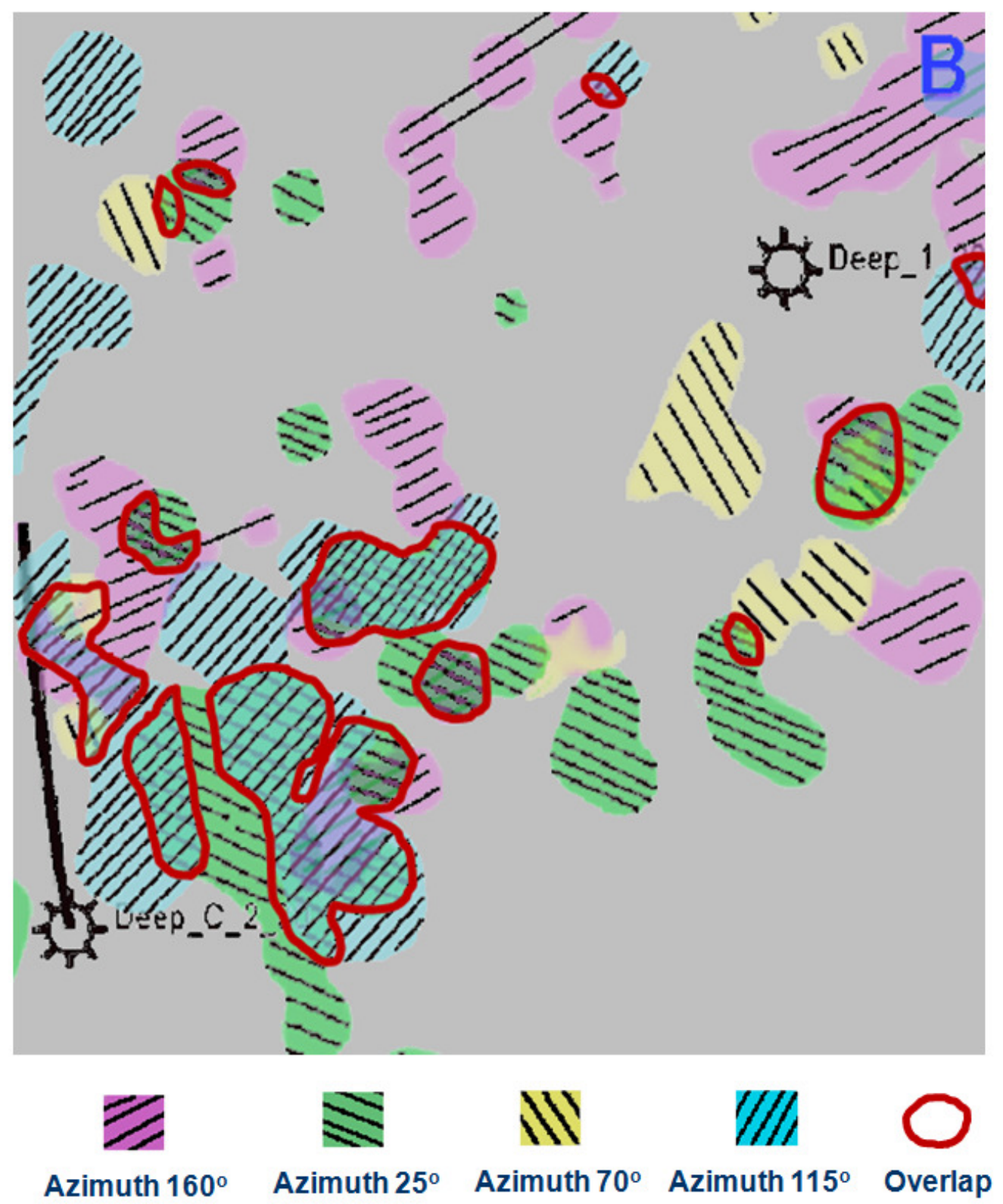

Figure 104: Combined Fracture Model resulting from the merging of Fracture Characterizations \#1 and \#2. 
Based on this study's theory these areas contain open, nature fractures. Fractures observed on azimuths 1 and 4 (central azimuths $25^{\circ}$ and $160^{\circ}$ ) would trend in a more east - west direction than those observed with azimuths 2 and 3, (central azimuths $70^{\circ}$ and $115^{\circ}$ ), which trend more north south. This suggests that the regional maximum horizontal stress direction is oriented east-west and holding open the fractures observed on azimuths $25^{\circ}$ and $160^{\circ}$. This stress direction would also tend to hold fractures associates with azimuths $70^{\circ}$ and $115^{\circ}$ more closed and may account for the notable lack of anomalies in these directions. This interpretation accounts for these azimuthal anomalies, associated fracture trends, their directions and locations and the increased amplitudes observed on the 4D Monitor survey that are believe due to the injected $\mathrm{CO}_{2}$. 

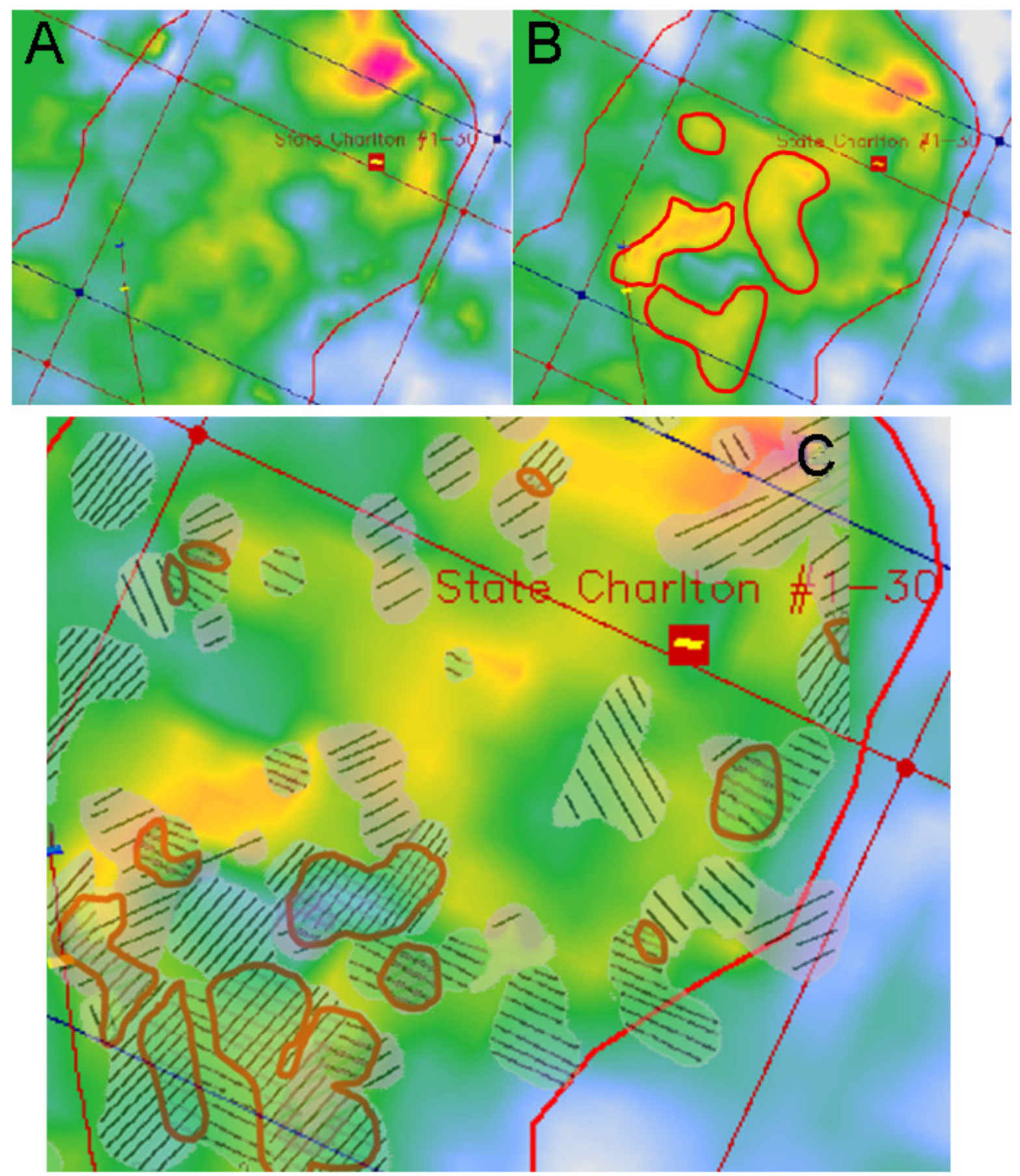

Figure 105: Comparison of the Baseline (A) and Monitor (B) surveys at time slice $15 \mathrm{~ms}$ below flatten A2Carbonate and the location of areas with high reflection strength from all four azimuthal iso-frequency volumes $(\mathbf{C})$.

Figure 105 shows a comparison between time slice $15 \mathrm{~ms}$ below the flattened A2Carbonate on the Baseline survey (A) and the Monitor survey (B). A significant amount of increased amplitude 
strength can be seen east and southeast of the $\mathrm{CO}_{2}$ injection point. These are circled in red on the Monitor survey (B). These areas, which include the previously discussed "Jet", are interpreted as the complete flow path of the $\mathrm{CO}_{2}$ at this level within the reservoir. This indicates that after being injected deep in the reef, the $\mathrm{CO}_{2}$ flows upwards to the top of the reef and then flows east northeast and southeast. Zones of increased amplitude can be seen in these areas on the Monitor survey (B). Area 1, referred to previously as the "Jet", flows to the east northeast from the injection point. This area contains the greatest amplitude increase and is, therefore, believed to contain large amounts of injected $\mathrm{CO}_{2}$. Based on the results of this study this area is interpreted as containing a zone of open, natural fractures that trend east northeast which act as conduits for the movement of the $\mathrm{CO}_{2}$ away from the injection point.

A portion of this area is contained by a fracture anomaly identified on the $15 \mathrm{~Hz}$ isofrequency volume for azimuth $4,\left(160^{\circ}\right)$. The orientation of these fractures would be in the same direction as the orientation of the "Jet", east northeast. This relationship suggests that the $160^{\circ}$ azimuthal volume is detecting the open fracture system that is acting as the conduit for some of the $\mathrm{CO}_{2}$ as it leaves the vicinity of the injection point. However, the anomaly on the Monitor survey is longer; covering a larger area than the anomaly seen on azimuth $160^{\circ}$, see Figures 102 and 103 . As noted previously the azimuthal volumes were developed from the Baseline survey, acquired before the initiation of $\mathrm{CO}_{2}$ injection and 3.5 years before the acquisition of the Monitor survey which displays the high amplitude "Jet". The two surveys were acquired during different times of the year, the Baseline survey in the spring and the Monitor survey in the fall. Although care was taken to acquire both surveys in an essentially similar manner the near surface conditions might have been different with water from snow melt permeating the surficial glacial deposits during the spring but not the fall. This may account for the difference in the anomalies sizes.

The area of increased amplitudes on the Monitor survey, designated area 2 on Figure 105(B), corresponds with a large portion of a fracture anomaly observed on the $15 \mathrm{~Hz}$ iso-frequency volume 
for azimuth $25^{\circ}$. Fractures detected by this azimuth would be oriented southeast. $\mathrm{CO}_{2}$ flow along these fractures would move towards the southeast from the injection point. The size and shape of the two anomalies appear to correspond.

Some correspondence can also be seen between area 3 on Figure 105(B) and a portion of a fracture anomaly observed on the $15 \mathrm{~Hz}$ iso-frequency volume for azimuth $25^{\circ}$. However in this instance the amount of correspondence is not as high. The small area to the north, area 4, does occur in the same vicinity as multiple fracture anomalies but is not coincident. 


\section{CHAPTER 8 - SUMMARY of FINDINGS and CONCLUSIONS}

\subsection{Summary of Findings}

I initially began this research as part of a study I proposed to the US Department of Energy, NETL, which they funded. This portion of the research was focused on determining if a 4D p-wave seismic survey could be used to monitor the flow of $\mathrm{CO}_{2}$ within a carbonate reservoir. During this portion of the research a number of relationships were observed between certain data types and the results from various analyses. These suggested frequency attenuation could be observed in the data set and used to identify zones of increased matrix porosity within the Charlton 30/31 reef. This concept is supported by a large amount of theoretical and experimental data in publications I mention in Chapter 2 and many others. However, little to no published data exists concerning attempts to apply this concept in the analysis of surface seismic data. Some of the support for this concept developed during this portion of the research is based on the findings from previous analyses also performed during the study. In other instances separate evidence has been developed. As a result of this investigation I believe that a significant amount of evidence was developed indicating that zones of frequency attenuation could be observed within the Charlton 30/31 reef and that these zones corresponded with zones of drained, high flow within the matrix.

As a result of the first portion of this study I decided to continue the investigation into this theory after the successful completion of the project for the US Department of Energy. This second portion of the research was designed to illustrate applications of a separate analytical approach. This second approach is largely independent of the first. The second analytical approach uses analysis and

interpretation of variable azimuth volumes extracted from the 3D seismic data over the field. This analysis is undertaken in an attempt to validate the findings obtained in the initial investigation presented in Chapters 2 through 4 and to extend the interpretation methodology to include the detection of open, natural fracture systems. During this second portion of the research, azimuthal seismic volumes developed from the Baseline 3D survey were analyzed with a number of different 
methods and compared with findings previously developed during this research, such as the analysis of the Monitor 3D survey.

A summary of these two separate but interlinked portions of this research is presented in the next two sections.

\subsection{D Seismic Based Analyses}

The initial portion of the project was conducted in order to determine if 4D surface P-wave seismic could be utilized to monitor the movement of $\mathrm{CO}_{2}$ through a carbonate reservoir at a depth of approximately 1 mile below the surface of the earth. During this portion of the study only normal, full azimuth seismic volumes were evaluated. The results of this portion of the study indicated that zones of frequency attenuation were being observed within the reservoir in the Baseline 3D seismic volume and that these zones were associated with zones of drained, high fluid flow. This conclusion is based on the following evidence.

- Instantaneous Frequency to Well Log Porosity Relationship: Neutron porosity logs blocked on intervals corresponding to 2 millisecond time slices were compared with multiple seismic attributes a relationship between high porosity and low frequency (indicated by low instantaneous frequency) was suggested. This is discussed in Chapter 4 and the relationship illustrated in Figure 50.

- Production History Matched Dynamic Model: Using this instantaneous frequency to $\log$ porosity relationship a static model of the reservoir was constructed. Zones of low frequency were interpreted and the relationship shown in Figure 50 was applied within these zones to deterministically distribute porosity within them. These contained porosities of $5 \%$ and higher. Outside of these zones a stochastic method was used to distribute porosity through the static model. These values were less than $5 \%$. This blended deterministic/stochastic static model was then used in a reservoir simulation, 
which was also production history matched. During the production history matching it was determined that although some slight adjustment to the bulk reef porosity was needed the porosity distribution within the reef was valid. This is explained in Figure 58 of Chapter 4. This reservoir simulation was based on a static model in which the porosity distribution was based on the frequency analysis. If this distribution was incorrect the actual oil production obtained by the different wells drilled into the field would not match those predicted by the simulation. The fact that a good production history match was obtained validates the porosity distribution which was based on the frequency analysis.

- 4D Survey Difference Mapping: Given that the production history match validated the $3 \mathrm{D}$ seismic survey derived porosity model, an additional reservoir simulation was undertaken to predict the distribution of the injected $\mathrm{CO}_{2}$ in the reservoir. Various well combinations were simulated in order to optimize enhanced oil recovery efforts in the field. This predictive reservoir simulation was run forward to the date when the 4D Monitor Survey was scheduled to be acquired in order to predict the location of the injected $\mathrm{CO}_{2}$ at that time. The 4D Monitor Survey was acquired on schedule, processed and then interpreted. The interpretation indicated that areas of increased amplitude existed within the Monitor survey at the top of the reef in a number of locations. A number of these corresponded to areas indicated by the forward looking, predictive reservoir simulation to contain $\mathrm{CO}_{2}$ for this time interval. This correspondence between the predictive reservoir simulation, developed from the interpretation of the Baseline survey, and the increased amplitudes on the Monitor survey indicates that the porosity distribution developed from the frequency analysis was accurate. 
- Drilling of the State Charlton 4 - 30 Well: Prior to the drilling of the State Charlton 4 - 30 in 2006 I predicted that the well would not encounter any porosity greater than $5 \%$. This prediction was made using the frequency to porosity relationship described in the first bullet item. Once the well was drilled and logged it was discovered that a zone of just over 5\% porosity existed in the middle of the reef which was less than 6 feet thick. Since a layer of this thickness was below the resolution of the seismic data this zone would not have been detectable and so the prediction made using the seismic data alone (the instantaneous frequency volume) was judged to be accurate.

- Drilling of the Larson 3-31 well: The Larsen 3-31 well was drilled in May of 2008 in the southern portion of the reef. Once again prior to the drilling of this well I examined the instantaneous frequency volume in the area where the well was to be drilled and predicted that it would encounter a zone of high porosity (higher than 5\%) approximately half way through the reef. This was drilled as planned and logged. The porosity logs obtained within the reef all appeared to have abnormally high readings. Higher than expected porosity reading were obtained in non-reservoir rocks above and below the reservoir. For this reason the most pessimistic of the porosity logs, the porosity calculated from the density readings, was used for comparison to with the seismic based prediction. Of the 22 intervals found to be above 5\% porosity 19 of them were below seismic resolution. Of the remaining 3 zones which were thick enough to be seismically resolvable, two occurred within the zone predicted by the frequency analysis. Additionally, although the borehole was drilled with 7 and 7/8 inch diameter drill bit an examination of the caliper log indicated that there was only one location where the hole diameter washed out significantly and exceeded 10 inches. This location corresponded to the top of the low frequency zone seen on the seismic and is 
interpreted as another indication of higher porosity. The remaining high porosity zone should have been seismically resolvable but was not recognized by the frequency analysis. Since two of the three high porosity zones at this location were predicated by the frequency analysis this result were judged to be a success.

During the portion of this research performed for the US Department of Energy results from the above lines of evidence suggested that frequency attenuation was an indication of high matrix porosity within the field (the Charlton 30/31 reservoir). The lines of evidence bulleted above are independent of one another, use different data types and support the theory proposed in this research. The well log data cross-plotted with the frequency data is independent of the field's primary production data and yet the production history matched reservoir simulation indicates that the porosity distribution developed using the frequency analysis was valid. The increased amplitudes observed on the Monitor survey of the 4D seismic data correlate with the location of the injected $\mathrm{CO}_{2}$ predicted by the forward-looking, predictive reservoir simulation, which was developed using the frequency analysis of the Baseline survey. Finally two separate wells, the State Charlton $4-30$ and Larsen 3 - 31 wells, were drilled into two different portion of the reef and encountered zones of high porosity predicted by the frequency analysis.

With the results of 5 separate analyses supporting the theory that low frequencies indicate zones of high porosity due to frequency attenuation it is difficult to justify that this may be all coincidental. Based on these results I believe that the high porosity zones within the reef, which also have high permeability associated with them, cause the attenuation of higher frequencies within the seismic signal leaving the low frequency component to dominate. This effect was initially predicated by Biot's initial publications in the mid 1950s and has been supported by a significant amount of theoretical and experimental work by other investigators since then. 


\subsection{Azimuthal Seismic Based Analyses}

The results of the first portion of this research indicated that careful frequency analysis, developed with an understanding of the frequency content of the seismic data set, could indicate where zones of low frequency existed in the reservoir. These low frequency zones were shown to correlate with zones of high porosity (and corresponding high permeability) by direct correlation with low frequency data and other evidence described in the previous section. A significant amount of published theoretical and experimental data supports concept that these low frequency zones are the result of the attenuation of the higher frequencies within the seismic signal.

Since the analysis performed during the first portion of this research was conducted using normal 3D seismic data, which was obtained and processed using all source - receiver pairs from all directions and angles (a "full-azimuth" 3D), the type of porosity that would be detected by this method would be matrix porosity, (see Figure 16 in Chapter 2). While the analysis performed produced good correspondence between drained, high flow zones within the matrix and zones of increased amplitudes observed in the 4D Monitor survey some variations were observed. Most notable of these variations is the anomaly believed to be a linearly aligned drained, high flow system, referred to as "The Jet", (see Figure 67 in Chapter 4). Based on concepts put forth by Colin Sayers with regard to the effect of open fractures and shear wave birefringence observed on azimuthal VSP data I believed that I could extend my theory to include directional frequency changes as a result of directionally aligned open fracture trends. I theorized that the variations observed in the increased amplitudes of the 4D Monitor survey could be explained through the analysis of azimuthally binned 3D seismic data. I also proposed that frequency attenuation could be identified through the interpretation of iso-frequency volumes developed through spectral decomposition. This has been the focus of the second portion of this research.

Four azimuthal volumes were developed using the same angular swath $\left(45^{\circ}\right)$ from the original 3D survey acquired in March of 2004. These had been developed during the research 
conducted for the US Department of Energy but had not been analyzed during that portion of the study. Spectral decomposition was performed on these four azimuthal volumes and seven isofrequency volumes were developed from each azimuthal volume. These were all developed from volumes that were flattened on the A2Carbonate in order to establish a datum of reference close to the reservoir in order to make the comparison between volumes more accurate. A number of analyses were performed during this second portion of the research in an effort to confirm the location of drained, high flow systems within the matrix located during the DOE study and to determine the location and direction of zones of open, natural fractures within the reef. A number of findings were made during this portion of the research.

- Azimuthal and Iso-frequency Normalization: Various comparisons were made of azimuthal iso-frequency data in order to determine if any inconsistencies may exist within the data set or if any patterns could be discerned within them. Normalization was performed on these volumes by both direction and frequency. During this portion of the research Azimuthal Sum Volumes, Iso-frequncy Sum Volumes, Selected Frequency Fraction (SFF) Iso-frequency Volumes, and All Azimuth Fraction (AAF) Iso-frequency Volumes were produced and compared. Little difference was found between the Selected Frequency Fraction (SFF) Iso-frequency Volumes and the original azimuthal iso-frequency volumes indicating that there were no software produced inconsistencies and that the original azimuthal iso-frequency volumes could be used in difference mapping.

- Frequency Attenuation: Iso-frequency Sum Difference Volumes were developed by subtracting the Iso-frequency Sum Volume for $55 \mathrm{~Hz}$ from the Iso-frequency Sum Volume for $15 \mathrm{~Hz}$, (see Figure 85 in Chapter 6). The patterns observed in this difference volume show a fair to good correspondence with the locations of the injected 
$\mathrm{CO}_{2}$ predicted by the forward-looking, predictive reservoir simulation. Since it was shown during the first portion of this research that the predictive reservoir simulation and the increased amplitudes observed in the 4D Monitor survey were aligned this would indicate that the Iso-Frequency Difference Volume was detecting zones of high matrix porosity in the original 3D survey. An exact alignment between area of high Isofrequency Difference and the predicted $\mathrm{CO}_{2}$ locations does not occur but this is expected as frequencies other than $55 \mathrm{~Hz}$ may also be attenuated in these areas and may not be pronounced in the $55 \mathrm{~Hz}$ to $15 \mathrm{~Hz}$ difference volume.

- Iso-Frequency Lineaments: Examination of various Azimuthal Iso-frequency volumes identified a number of linear features. These were typically fairly subtle, low amplitude, features. Many of these lineaments were clearly acquisition related as they were aligned with the volume's in-line and cross-line directions, (see Figure 85 in Chapter 6). However, a few of these were not oriented in the in-line or cross-line directions and are located near the amplitude anomaly referred to as "The Jet", (see Figure 97). These are observed on the All Azimuth Fraction (AAF) Iso-frequency volumes and may be related to fracture trends in this area since they are oriented in the same direction as the "The Jet", however, they do not display any significant strengthening in amplitude and, therefore, do not display any frequency attenuation.

- Azimuthal-Based Fracture Detection and Fracture Characterization: Pairs of low frequency azimuthal iso-frequency volumes that were normal to one another were examined and compared. In theory azimuthal volumes that were perpendicular to any open fracture trends would show increased amplitudes in those fracture zones in the lower iso-frequency volumes. Those azimuthal volumes that were parallel to those fracture trends would not show any increased amplitudes. The two pairs of azimuthal 
volumes selected for comparison were azimuths $25^{\circ}$ and $115^{\circ}$ and azimuth $70^{\circ}$ and $160^{\circ}$. Both azimuths $160^{\circ}$ and $25^{\circ}$ show strong amplitudes on the $15 \mathrm{~Hz}$ iso-frequency volumes in the vicinity of the injection point and the EOR well. Correspondingly the azimuths normal to these, azimuths $70^{\circ}$ and $115^{\circ}$, show significantly lower amplitudes on the $15 \mathrm{~Hz}$ iso-frequency volumes in the same area. These areas of increased amplitude on these four azimuthal $15 \mathrm{~Hz}$ iso-frequency volumes were mapped at $15 \mathrm{~ms}$ below the top of the A2Carbonate reflection event. These comparisons are shown in Figure 101 (for azimuths $160^{\circ}$ and $70^{\circ}$ ) and Figure 102 (for azimuths $25^{\circ}$ and $115^{\circ}$ ) and the collection of all azimuths is shown in Figure 103. The dominance of azimuths $160^{\circ}$ and $25^{\circ}$, both the more east-west oriented azimuths, is interpreted to be the location of fracture trends that are held open by an east-west oriented maximum horizontal stress direction. The observation that two azimuths, $45^{\circ}$ apart, indicate open fracture trends suggests that the stress anisotropy within the reef is not large. This would be expected since carbonates tend to have high strength with regard to stress and are generally more stress isotropic in nature.

- Interpreted Fracture Trend Alignment with 4D Monitor Survey Amplitudes: The fracture trends interpreted from the analysis of the $15 \mathrm{~Hz}$ azimuthal Iso-frequency volumes, which were developed from the original 3D seismic survey, were compared to the increased amplitudes observed on the 4D Monitor survey (see Figure 104 in Chapter 7). This comparison indicated a correspondence in a number of areas. Azimuth $160^{\circ}$ dominates the area immediately to the east of the injection point. Fractures detected by this azimuth would trend to the east northeast, the same direction as the feature referred to as "The Jet". However, only the central portion of "The Jet" is covered by the azimuth $160^{\circ}$ anomaly. The area to the southeast of the injection point, 
labeled area 2 in the B portion of Figure 102, corresponds to the largest area of fracturing indicated by azimuth $25^{\circ}$. Fractures detected by this azimuth would be oriented southeast and would act as conduits for the injected $\mathrm{CO}_{2}$ to move to the southeast instead of directly towards to EOR well. Also in this area is another anomaly associated with azimuth $160^{\circ}$, and some overlap occurs with anomalies from azimuth $25^{\circ}$. Since the overlap zones show strong low frequency on more than one azimuth this may be indicating zones of high matrix porosity, although this is not observed on the other two azimuth $70^{\circ}$ and $115^{\circ}$.

During this second portion of this research effort multiple analytical methods were investigated in an attempt to detect open fracture systems within the reef, identify their orientation and distinguish these from the drained, high flow systems in the matrix. The results from these methods suggest that this was achieved. 1) Difference volumes developed between the $15 \mathrm{~Hz}$ and 55 Hz Iso-frequency Sum Volumes show a correspondence between areas of strong low frequency and zones of increased amplitudes on the 4D Monitor survey. 2) Azimuthal volumes normal to one another indicate significant differences in low frequency reflection strength, as predicted by this theory. 3) Lineaments are observed on certain azimuthal iso-frequency volumes that align with the location and orientation of the 4D Monitor amplitude anomaly referred to as "The Jet", although amplitude strengthening is not observed. This may be due to the nature of the way these volumes were normalized. 3) Areas where the $15 \mathrm{~Hz}$ iso-frequency volumes contain strong amplitudes for azimuths $160^{\circ}$ and $25^{\circ}$ correspond with areas of increased amplitude on the 4D Monitor survey. Additionally, the orientation of these fractures explains the movement of $\mathrm{CO}_{2}$ away from the injection point.

It should be noted that the correspondence between the $15 \mathrm{~Hz}$ azimuthal iso-frequency volumes and the increased amplitudes of the 4D Monitor survey is not exact. This may be due to one or more of the following; 
- The aziumthal volumes were developed from the first 3D survey that was acquired in March of 2004. This volume was processed before the second 3D survey was available and so it was not processed with the consideration of being part of a 4D survey. The first and second 3D surveys were processed as a 4D survey once both were available and the Monitor survey was developed. These two surveys (the azimuthal volumes developed from the first 3D survey and the Monitor survey from the 4D) may have subtle differences in the way they were processed that may account for these differences observed between the anomaly on the $160^{\circ}$ azimuth and "the Jet".

- The azimuthal volumes were developed during my early research into azimuthal analysis and they were developed using a constant angular swath of $45^{\circ}$ for each sector (azimuth). This, most probably, resulted in some slight variation in azimuthal fold between each sector. This may also explain why some areas of noncorrespondence occur between these two data sets.

\subsection{Conclusions}

The research presented in this document is the result of eight years worth of research, originally initiated as part of a study performed for the US Department of Energy and then extended with additional research. The primary goal of this study was to develop a workflow that could be used to characterize the drained, high flow systems within a subsurface reservoir using traditional pwave seismic data before drilling an expensive wellbore. This research was also designed to test this workflow by determining if azimuthal iso-frequency data could be used to detect the drained, high flow systems within the matrix as well as distinguish these from zones of open fractures and determine the fracture trend orientations. To the best of my knowledge this approach has not been attempted previously. 
The results of this investigation strongly suggest that the study has been, to a large part, successful. In the oil business we have a saying, "The proof comes when the drill bit cuts the formation". In geophysics in the industry we consider well data to be "hard data". During this study this occurred twice with the drilling of the State Charlton $4-30$ and the Larsen $3-31$ wells. Those wells found the porosity/permeability systems that I predicted using these methods. Unfortunately the other observations that have been made since the drilling of those two wells during this study have yet to be tested to that same degree of "hardness". However, a significant amount of "soft data" (seismic data) support many of the conclusions drawn during this investigation.

The results of this research could have significant impact on various industries that are required to drill into the Earth's subsurface, such as the oil and gas industry and the environmental industry. The successful placement of expensive boreholes, whether to extract hydrocarbons or sequester wastes, is dependent upon drilling into zones with high storage capacity. The method described in this research not only provides a means of detecting and mapping the drained, high flow systems within the reservoir, but is also able to determine whether these zones are associated with matrix or fracture porosity systems and the direction of the open fracture trends. This allows a reasonably accurate characterization of a reservoir's drained, high flow systems to be developed prior to the drilling of expensive boreholes through the interpretation of azimuthal P-wave seismic data. This capability could significantly increase the cost effectiveness of borehole placement within carbonate reservoirs. 


\section{REFERENCES}

Ali, Aamir, 2011, Seismic characterization of reservoirs with multiple fracture sets using velocity and attenuation anisotropy data, Journal of Applied Geophysics 75 (2011) 590-602

Adam, Ludmila, 2009, Seismic Wave Attenuation in Carbonates, Journal of Geophysical Research, Vol. 114

Akbar, Nabil, Dvorkin, Jack, Nur, Amos, 1993, Relating P-wave attenuation to permeability, Geophysics, Vol. 58, no. 1, p. 20-29

Akbar, Nabil, 1994, Seismic signatures of reservoir transport properties and pore fluid distribution, Geophysics , Vol. 59, No. 8 (August 1994); P. 1222-1236

Bai, Mao, 1993, Multiporosity / Multipermeability Approach to the Simulation of Naturally Fractured Reservoirs, Water Resources Research, Vol. 29, No. 6, pages 1621 - 1633

Batzle, M., Han, D.H., Castagna J., 1996, Attenuation and Velocity Dispersion at Seismic Frequencies, 66th Annual SEG International Meeting (Denver, 11/10-15/96) Expended Abstract, v.2, pp.1687-1690

Batzle, M., 1999, Fluids and frequency dependent seismic velocity of rocks, SEG Expanded Abstracts

Batzle, M., 2003, Macro-flow and Velocity Dispersion,

Batzle, M., 2005, Seismic Attenuation: Observations and Mechanisms, SEG 2005 Annual Meeting

Batzle, M., Han, D.H., and Hofmann, R., 2006, Fluid mobility and frequency-dependent seismic velocity — Direct measurements, Geophysics, vol. 71, no. 1, p. N1-N9

Berryman, James, 2007, Seismic waves in rocks with fluids and fractures, Geophys. J. Int. (2007) $171,954-974$ 
Berryman, James, 2009, Aligned vertical fractures, HTI reservoir symmetry and Thomsen seismic anisotropy parameters for polar media, Geophysical Prospecting, 2009, 57, 193-208

Biot, M. A.: Theory of Elasticity and Consolidation for a Porous Anisotropic Solid," Journal of Applied Physics (February 1955) 26, No. 2, p. 182 to185.

Biot, M. A.: Theory of Propagation of a Porous Viscoelastic Anisotropic Solid, Journal of Applied Physics (May 1956) 27, No. 5, P. 458 - 467

Born, W. T., 1941, The Attenuation Constant of Earth Materials

Brajanovski, M., Gurevich, B., 2003, Frequency dependent anisotropy of porous rocks with aligned fractures., 16th ASEG Geophys. Conf., Brisbane, Australia, 2/16 - 19/2003 (Abstract only)

Brajanovski, M., Gurevich B., Lambert, G., 2003, Attenuation and dispersion of compressional waves in porous rocks with aligned fractures., 73rd Annu. SEG Int. Mtg. Expanded Abstr. Biog. V. 2, pp. 1620-1623, 2003.

Brajanovski, M., 2006, Cross-over frequencies of seismic attenuation in fractured porous rocks, SEG 2006 Annual Meeting

Bullock, Terra E., 1999, The Investigation of Fluid Properties and Seismic Attributes for Reservoir Characterization, Michigan Technological University, Thesis

Carcione, J., and Picotti, S., 2006, P-wave seismic attenuation by slow-wave diffusion: Effects of inhomogeneous rock properties, Geophysics, vol. 71, no. 3, p. O1-O8

Carcione, Jose M., 2010, Computational poroelasticity - A review, Geophysics ,Vol. 75, No. 5

Cercone, Karen Rose, and Lohmann, Kyger C., 1987, Late Burial Diagenesis of Niagaran (Middle Silurian) Pinnacle Reefs in Michigan Basin: Bulletin of the American Association of Petroleum Geologists, Vol. 71, No. 2, PP 156-166 
Chakraborty, Avijit and Okaya, David, 1995, Frequency-time decomposition of seismic data using wavelet-based methods, Geophysics, vol. 60, no. 6, p. 1906-1916

Chapman, Mark, 2003, Frequency-dependent anisotropy due to meso-scale fractures in the presence of equant porosity, Geophysical Prospecting, 2003, 51, 369-379

Chapman, Mark, 2006, Use of spectral decomposition to detect dispersion anomalies associated with gas saturation. EAGE 68th Conference \& Exhibition — Vienna, Austria, 12 - 15 June 2006

Castagna, J. P., and Sun, S., 2006, Comparison of spectral decomposition methods, first break volume $24, \mathrm{p} 75$ to 79 .

Coats, K. H., Thomas, L.K. and Pierson, R.G., 1995, Compositional and Black Oil Reservoir Simulation, SPE-29111-MS, 13th SPE Symposium on Reservoir Simulation Held In San Antonio, TX, U.S.A., 12-15 February 1995.

Detournay, Emmanuel, 1993, Fundamentals of Poroelasticity, Chapter 5 in Comprehensive Rock Engineering: Principles, Practice and Projects, Vol. II, Analysis and Design Method

Dilay, A., 1995, Spectral analysis applied to seismic monitoring of thermal recovery, The Leading Edge, November, 1995

Dix, C. Hewitt, 1955, Seismic Velocities From Surface Measurements, Geophysics, Vol. XX, No. 1

Dvorkin, J., Nur, A., 1991, Pore Fluid : Attenuation, Permeability, Compressibility, 61st Annu. SEG Int. Meeting. (Houston, 11/10-14/91) Extended Tech. Programs Abstr. Biogr. v.2, pp.1031-1033

Dvorkin, Jack, 1992, The dynamics of viscous compressible fluid in a fracture, Geophysics VO, L. 57 , No. 5

Dvorkin, Jack, Nur, Amos, 1993, Dynamic poroelasticity: A unified model with the squirt and the Biot mechanisms, Geophysics, vol. 58, no. 4, p. 524-533 
Dvorkin, Jack, 1994, The squirt-flow mechanism: Macroscopic description, Geophysics , Vol. 59, No. 3

Dvorkin, Jack, Mavko, Gary, Nur, Amos, 1995, Squirt flow in fully saturated rocks, Geophysics, vol. 60, no. 1 , p. $97-107$

Dvorkin, Jack, 1999, Diagnosing high-porosity sandstones: Strength and permeability from porosity and velocity, Geophysics, Vol. 64, No. 3

Dvorkin, Jack, and Mavko, Gary, 2006, Modeling attenuation in reservoir and nonreservoir rock, The Leading Edge, February 2006

Endres, A. and Knight, R., 1997, Incorporating pore geometry and fluid pressure communication into modeling the elastic behavior of porous rocks., Geophysics, vol. 62, no. 1, p. 106-117

Fang, Xinding, 2010, Sensitivity analysis of fracture scattering

Futterman, Walter I., 1962, Dispersive Body Waves, Journal of Geophysical Research, Vol. 67, No. 13

Gelinsky, Stephan, 1998, Anisotropic permeability in fractured reservoirs from integrated acoustic measurements, 1998 SEG Expanded Abstracts

Gill, D., 1973, Stratigraphy facies, evolution and diagenesis of productive Niagaran Guelph reefs and Cayugan Sabkha deposits, the Belle River Mills gas field, Michigan Basin: Unpublished Ph.D. Thesis, University of Michigan, 275 p.

Gill, D., 1977, Salina A-1 Sabkha Cycles and the late Silurian Paleogeography of the Michigan Basin: Journal of Sedimentary Petrology, Vol. 47, No. 3, PP 979-1017

Gill, D., 1979, Differential Entrapment of Oil and Gas in Niagaran Pinnacle-Reef Belt of Northern Michigan, Bulletin of the American Association of Petroleum Geologists, Vol. 63, No. 4, PP 608-620 
Gill, D., 1994, Niagaran Reefs of Northern Michigan, Part I: Exploration Portrait: Journal of Petroleum Geology, Vol. 17(1), pp. 99-110

Goto, Hiroyuki, 2010, Definition of Normalized Energy Density and Its Application to Direct Estimation of Damping Property, 4th IASPEI / IAEE International Symposium

Grammer, G. Michael, 2006, Phase II (Year 2) Summary of Research - Establishing the Relationship between Fracture-Related Dolomite and Primary Rock Fabric on the Distribution of Reservoirs in the Michigan Basin, DOE Award Number: DE-FC26-04NT15513

Grammer, G. Michael, 2007, Summary of Research through Phase II/Year 2 of initially approved 3 Phase/3 Year Project - Establishing the Relationship between Fracture-Related Dolomite and Primary Rock Fabric on the Distribution of Reservoirs in the Michigan Basin, Final Scientific/Technical Report: DE-FC26-04NT15513

Grammer, G. Michael, 2009, Practical Synergies for Increasing Domestic Oil Production and Geological Sequestration of Anthropogenic CO2: An Example from the Michigan Basin, AAPG Studies in Geology 59, p. 689-706

Grammer, G. Michael, 2010, Reservoir Characterization of Silurian (Niagaran) "Pinnacle" Reefs in the Michigan Basin, Search and Discovery Article \#50286

Gregory, A. R., 1977, Volume M 26: Seismic Stratigraphy--Applications to Hydrocarbon Exploration, Pages 15 - 46,

Gridley, James, and Partyka, Greg, 1997, Processing and interpretational aspects of spectral decomposition, SEG Technical Program Expanded Abstracts -- 1997 -- pp. 1055-1058

Gurevich, B., 2000, Effect of Fluid Viscosity on Elastic Wave Attenuation in Porous Rocks, Geophysics vol.67, no.1, pp.264-270, Jan.-Feb. 2002 
Gurevich, Boris, 2009, P-wave dispersion and attenuation in fractured and porous reservoirs poroelasticity approach, Geophysical Prospecting, 2009, 57, 225-237

Hall, M., 2006, Predicting Bed Thickness with Cepstral Decomposition., The Leading Edge, February 2006

Haugen, Geir U., 2000, The echo of a fault or fracture, Geophysics , Vol. 65, No. 1

Han, De-hua 2004, Gassmann's equation and fluid-saturation effects on seismic velocities, Geophysics , Vol. 69, No. 2

Henderson, J., Purves, S. J., and Leppard, C., 2007, Automated delineation of geological elements from 3D seismic data through analysis of multichannel, volumetric spectral decomposition data., First Break, vol. 25, March 2007

Hofmann, Ronny, Frequency Dependent Elastic and Anelastic Properties of Clastic Rocks, Colorado School of Mines, Dissertation.

Hudson, J.A., 1990, Attenuation due to second-order scattering in material containing cracks, Geophys. J. Int. v. 102, no. 2, pp. 485-490, Aug. 1990

Indiana University Paleontology Seminar, 1980, Stratigraphy, Structure, and Zonation of Large Silurian Reef at Delphi, Indiana: Bulletin of the American Association of Petroleum Geologists, Vol. 64, No. 1, PP 115-131

Indiana University Paleontology Seminar, 1976, Silurian Reef Complex, Rockford, Ohio: Constitution, Growth, and Significance: Bulletin of the American Association of Petroleum Geologists, Vol. 60, No. 3, PP 428-451 
Ingels, Jerome J. C., 1963, Geometry, Paleontology, and Petrography of Thornton Reef Complex, Silurian of Northeastern Illinois: Bulletin of the American Association of Petroleum Geologists, Vol. 47, No. 3, PP 405-440

Jakobsen, Morten, 2005, The effects of drained and undrained loading on visco-elastic waves in rock-like composites, International Journal of Solids and Structures 42 (2005) 1597-1611

Jodry, Richard L., 1969, Growth and Dolomitization of Silurian Reefs, St. Clair County, Michigan: Bulletin of the American Association of Petroleum Geologists, Vol. 53, No. 4, PP 957-981

Johnston. D. H., Toksoz, M. N., and Timur, A., 1979, Attenuation of seismic waves in dry and saturated rocks: II, Mechanisms: Geophysics, v. 44, p. 691 - 711.

Jones, Terry, 1980, Effects of Temperature and Saturation on the Velocity and Attenuation of Seismic Waves in rocks: Applications to Geothermal Reservoir Evaluation, Proceedings, Sixth Workshop Geothermal Reservoir Engineering, Stanford University, Stanford, California, December $16-18,1980$

Kjartansson, Einar, 1979, Attenuation of Seismic Waves in Rocks and Applications in Energy Exploration, Stanford Exploration Project Report No. 23 and Stanford Rock Physics Project Report No. 8

Kolinsky, Petr, 2004, Surface Waves Dispersion Curves of Eurasian Earthquakes: The Sval Program, Acta Geodyn. Geomater.Vol.1, No.2 (134), 165-185

Kozlov, E. 2004, Pressure-dependent seismic response of fractured rock, Geophysics, vol. 69, no. 4, p. $885-897$

Labo, J., 1981, Exploring for Silurian-Niagaran Pinnacle Reefs in the Southern Michigan Basin, Oil and Gas Journal, June 22, 1981. 
Lei, Xinglin, 2011, Role of drainage conditions in deformation and fracture of porous

rocks under triaxial compression in the laboratory, Geophysical Research Letters, Vol. 38

Levin, Franklyn K., 1978, The Reflection, Refraction, and Diffraction of Waves in Media with Elliptical Velocity Dependence, Geophysics , Vol. 43, No. 3

Levin, Franklyn K., 1979, Seismic velocities in transversely isotropic media, Geophysics, Vol. 44, No. 5

Liu, E., et al, 2004, Modeling seismic wave propagation during fluid injection in a fractured network: Effects of pore fluid pressure on time-lapse seismic signatures., The Leading Edge. August, 2004

Liner, Christopher L., 2002, Phase, phase, phase, The Leading Edge, May 2002

Liner, Christopher L., 2009, Seismic dispersion, Seismos, http://seismosblog.blogspot.com/2009/09/seismic-dispersion.html

Liner, Christopher L., 2009B, Dispersion and Processing: Attenuation and

Anisotropy, Seismos, http://seismosblog.blogspot.com/2009/09/dispersion-and-processing-attenuation.html

Liu, Enru, 2005, Effects of fracture aperture and roughness on hydraulic and mechanical properties of rocks: implication of seismic characterization of fractured reservoirs, Journal of Geophysics and Engineering, 2 (2005) 38-47

Loizou, Nick, 2008, AVO analyses and spectral decomposition of seismic data from four wells west of Shetland, United Kingdom, Petroleum Geoscience, 14, 355-368.

Love, A. E. H., 1906, A Treatise on the Mathematical Theory of Elasticity,

Lubbe, R., 2006, A field investigation of fracture compliance., Geophysical Prospecting, 2006, 54, $319-331$ 
Lynn, H. B., Simon, K. M. and Beckham, W., 1997, Fracture detection, mapping and analysis of naturally fractured gas reservoirs using P-wave reflection seismic, 67th Ann. Internat. Mtg: Soc. of Expl. Geophys., 1210-1213.

Lynn, H. B., Campagna, D., Simon, K. M. and Beckham, W. E., 1999, Relationship of P-wave seismic attributes, azimuthal anisotropy, and commercial gas pay in 3-D P-wave multiazimuth data, Rulison Field, Piceance Basin, Colorado: Geophysics, Soc. of Expl. Geophys., 64, 1312-1346.

Lynn, H. B., 2004, The Winds of Change - Anisotropic Rocks, Their Prefered Direction of Flow and Their Associated Seismic Signatures., The Leading Edge, Nov and Dec, 2004

Martin, Nicolas W., 1996, Compressional velocity, seismic attenuation and permeability relationships for sandstones from WOSPP, CREWES Research Report - Volume 8 (1996)

Mavko, G., and Nur, A., 1979, Wave attenuation in partially saturated rocks: Geophysics, v. 44, No. 2, p. $161-178$

McCollum, B. and Snell, F. A., 1932, Asymmetry of Sound Velocity in Stratified Formations: Physics, vol. 2, issue 3, p $174-185$

Melhorn, Wilton N., 1958, Stratigraphic Analysis of Silurian Rocks in Michigan Basin: Bulletin of the American Association of Petroleum Geologists, Vol. 42, No. 4, PP 816-838

Mesolella, K. J., Robinson, J. D., McCormick, L. M. and Ormiston, A. R., Cyclic Deposition of Silurian Carbonates and Evaporites in Michigan Basin: Bulletin of the American Association of Petroleum Geologists, Vol. 58, No. 1, PP 34-62

Misaghi, Ali, 2010, A comparison of rock physics models for fluid substitution in carbonate rocks, Exploration Geophysics, 2010, 41, 146-154 
Mollhoff, M., 2010, Rock fracture compliance derived from time delays of elastic waves, Geophysical Prospecting, 2010, 58, 1111-1121

Montoya, P., et al, 2005, Definition of depositional geological elements in deep-water minibasins of the Gulf of Mexico using spectral decomposition in depth domain., SEG Technical Program Expanded Abstracts -- 2005 -- pp. 481-484

O'Connell, R. J., and Budiansky, B., 1977, Viscoelastic properties of fluid-saturated cracked solids: J. Geophys. Res., v. 82, p. 5719 GET

Morozov, Igor B., The mechanics of seismic attenuation, INGV, Annals of Geophysics.

Muller, Tobias M., 2004, Seismic attenuation and dispersion due to wave-induced flow in 3-D inhomogeneous porous rocks, SEG International Exposition and 74th Annual Meeting, Denver, Colorado, 10-15 October, 2004

Muller, Tobias M., 2010, Seismic wave attenuation and dispersion resulting from wave-induced flow in porous rocks - A review, Geophysics, Vol. 75, No. 5

Partyka, G., Gridley, J., and Lopez, J., 1999, Interpretational applications of spectral decomposition in reservoir characterization., The Leading Edge, March 1999

Misra, H C, 1965, Permeability of porous media to transient flow, thesis, 96 pp, 1965 (abstract only)

Najmuddin, Ilyas, 2001, Frequency Attenuation: A Fracture Indicator, Houston Geological Society Bulletin, March, p. 25-28

Nichols, D., 1989, Elastic Properties of Rocks with Multiple Sets of Fractures, SEG Expanded Abstracts 8, 471

Odebeatu, Emeka, 2006, Application of spectral decomposition to detection of dispersion anomalies associated with gas saturation, The Leading Edge, February 2006 
Pan, Y., 1999, Reservoir Analysis Using Intermediate Frequency Excitation, Dissertation, Stanford University

Palmer, I. D., Traviolia, M. L., 1980, Attenuation by squirt flow in undersaturated gas sands, Geophysics, vol. 45, no. 12, p. 1780-1792

Parra, Jorge O., 2002, Wave attenuation attributes as flow unit indicators, The Leading Edge, June, 2002

Pride, S. R., 2004, Seismic attenuation due to wave-induced flow, Journal of Geophysical Research, Vol. 109

Pyrak Nolte, Laura J., 1995, Wavelet Analysis of Velocity Dispersion of Elastic Interface Waves Propagating Along a Fracture, Geophysical Research Letters, Vol. 22, No. 11.

Quan, Youli, 1997, Seismic attenuation tomography using the frequency shift method, Geophysics, Vol. 62, No. 3

Rojas, Nelson A., 2008, Spectral Decomposition Applied to Time-Lapse Seismic Interpretation at Rulison Field, Garfield County, Colorado, Colorado School of Mines, Thesis.

Ruger, Andreas, 1997, P-wave reflection coefficients for transversely isotropic models with vertical and horizontal axis of symmetry, Geophysics, Vol. 62, No.3

Ruger, Andreas, 1998, Variation of P-wave reflectivity with offset and azimuth in anisotropic media, Geophysics, Vol. 63, No.3

Sayers, C. M., 1991, A Simple Technique for Finding Effective Elastic Constants of Cracked Solids for Arbitrary Crack Orientation Statistics, International Journal of Solids and Structures, Vol. 27. No. 6. pp. $671-680$ 
Sayers C. M., 1995, Microcrack-Induced Elastic Wave Anisotropy of Brittle Rocks, Journal of Geophysical Research, Vol. 100, No. B3

Sayers, C. M., 1997, Determination of anisotropic velocity models from walkaway VSP data acquired in the presence of dip, Geophysics, vol. 62, no. 3, p. 723-729

Sayers, C. M., 2008, The elastic properties of carbonates, The Leading Edge, August 2008

Sayers, C. M., 2009, The effect of mineralization on the ratio of normal to tangential compliance of fractures, Geophysical Prospecting, 2009, 57, 439-446

Sayers, C. M., 2009B, Seismic characterization of reservoirs containing multiple fracture sets, Geophysical Prospecting, 2009, 57, 187-192

Sharma, M. D., 2004, Wave propagation in a general anisotropic poroelastic medium with anisotropic permeability: phase velocity and attenuation, International Journal of Solids and Structures 41 (2004) 4587-4597

Spencer, T. W., Date unknown, Measurement and Interpretation of seismic attenuation 29GP developments in geophysical exploration methods (Elsevier Develop Ser) V 6, PP 71-109 Elsevier Applied Science Publishers ltd, New York, 1985 (ISBN 0-85334-344-6; 15 refs)

Schoenberg, Michael, 1986, Fluid and solid motion in the neighborhood of a fluid-filled borehole due to the passage of a low-frequency elastic plane wave, Geophysics , Vol. 51. No. 6

Schoenberg, Michael, 1991, Acoustic characterization of underground fractures, 1998 SEG Expanded Abstracts

Schoenberg, M., 1998, Acoustic characterization of underground fractures, SEG Technical Program Expanded Abstracts -- 1998 -- pp. 1624-1627 
Schoenberg, M., Dean S., and Sayers, C. M. 1999, Azimuth-dependent tuning of seismic waves reflected from fractured reservoirs, Geophysics. Vol. 64. No 4; P. 1160-1171

Schoenberg, M. and Nakagawa, S., 2006, Poroelastic modeling of seismic boundary conditions across a fracture, SEG Technical Program Expanded Abstracts -- 2006 -- pp. 2991-2995

Schoenberg, M., 2009, Vertically fractured transversely isotropic media: dimensionality and deconstruction, Geophysical Prospecting, 2009, 57, 169-185

Sena, Arcangelo G., 1991, Seismic traveltime equations for azimuthally anisotropic and isotropic media: Estimation of interval elastic properties, Geophysics , Vol. 56, No. 12

Seriff, A. J., 1991, P-SV reflection moveouts for transversely isotropic media with a vertical symmetry axis, Geophysics, Vol. 56. No. 8

Shapiro, Sergei A., 1999, Seismic signatures of permeability in heterogeneous porous media, Geophysics , Vol. 64, No.1

Sharma, M. D., 2007, Wave propagation in a general anisotropic poroelastic medium: Biot's theories and homogenisation theory, J. Earth Syst. Sci. 116, No. 4, August 2007, pp. 357-367

Shaver, Robert H., 1974, Silurian Reefs of Northern Indiana: Reef and Interreef Macrofaunas: Bulletin of the American Association of Petroleum Geologists, Vol. 58, No. 6, PP 934-956

Shaver, Robert H., 1977, Silurian Reef Geometry - New Dimensions to Explore: Journal of Sedimentary Petrology, Vol. 47, No. 4, PP 1409-1424

Shen, Feng, 1998, Seismic Characterization of Fractured Reservoirs, Massachusetts Institute of Technology, Dissertation.

Simo, J. A. and Lehmann, Patrick J., 2000, Diagenetic History of Pipe Creek JR. Reef, Silurian, North-Central Indiana, U.S.A.: Journal of Sedimentary Research, Vol. 70, No. 4, PP 937-951 
Somerstein, S. F., 1984, Radio-frequency geotomography for remotely probing the interiors of operating mini- and commercial-sized oil-shale retorts, Geophysics. Vol 49. No 8 (August 1984); P. $1288-130$

Spencer, James W.,1981, Stress Relaxations at Low Frequencies in Fluid-Saturated Rocks: Attenuation and Modulus Dispersion, Journal of Geophysical Research, Vol. 86, No. B3

Strobel, Michael L, 1991, Areal Extent, Hydrogeologic Characteristics, and Possible Origins of the Carbonate Rock Newburg Zone (Middle-Upper Silurian) in Ohio, OHIO J. SCI. 91 (5): 209-215, 1991

Sun, Langqiu F.,2009, Measuring velocity dispersion and attenuation in the exploration seismic frequency band, Geophysics, Vol. 74, No. 2

Taner, M. TURHAN, 1969, Velocity Spectra-Digital Computer Derivation and Applications of Velocity Functions, Geophysics, Vol 34, No. 6

Teng, Li, 1998, Seismic and Rock-Physics Characterization of Fractured Reservoirs, Stanford University, Dissertation

Thomsen, Leon, 1985, Biot-consistent elastic moduli of porous rocks: Low-frequency limit, Geophysics, Vol. 50, No. 12

Thomsen, Leon, 1986, Weak elastic anisotropy, Geophysics, Vol. 51, No. 10

Thomsen, Leon, 1988, Reflection seismology over azimuthally anisotropic media, Geophysics, Vol. 53, No.3

Todd, M. R. and Longstaff, W. J., The Development, Testing, and Application of a Numerical Simulator for Predicting Miscible Flood Performance, Journal of Petroleum Technology, July, 1972. 
Toelle, B., Carney, B. J. and Campbell, Richard, 2004, 2-D variance and blended attribute analysis of deep structures in Lincoln, Co., West Virginia, SEG Technical Program Expanded Abstracts -- 2004 -- pp. 541-543

Toelle and Pekot, 2006, Use of Instantaneous Frequency to Map Porosity in a Silurian Age Reef in Northern Michigan Basin in Preparation for $\mathrm{CO}_{2}$ Injection, Berkeley CO2SC Symposium.

Toelle, Yang and Imai, 2007, $\mathrm{CO}_{2}$ Sequestration Potential of the North Michigan Silurian Reef Trend, American Association of Petroleum Geologists (AAPG) Eastern Regional Meeting.

Toelle, Pekot and Mannes, 2007, $\mathrm{CO}_{2}$ EOR from a North Michigan Silurian Reef, SPE \# 111223-PP

Toelle, Pekot and Grammer, 2008, EOR Potential of the Michigan Silurian Reefs Using $\mathrm{CO}_{2}$, SPE \# 113843-PP

Toelle, B., 2009, Application of Time-Lapse Seismic Monitoring for the Control and Optimization of $\mathrm{CO}_{2}$ Enhanced Oil Recovery Operations, DOE Award No.: DE-FC26-04NT15425

Toksoz, M. N., Johnston. D. H. and Timur, A., 1979, Attenuation of seismic waves in dry and saturated rocks: I, Laboratory measurements: Geophysics, v. 44, p. 681 -690

Tsvankin, IIya, 1994, Nonhyperbolic reflection moveout in anisotropic media, Geophysics , Vol. 59, No.8

Tsvankin, Ilya, 1997, Reflection moveout and parameter estimation for horizontal transverse isotropy, Geophysics, Vol. 62, No. 2 (March-April 1997); P. 614-629, 7 Figs.

Varga, Audrey L. and Grammer, G. Michael, 2011, Evaluating the Controls on Reservoir Quality and Heterogeneity of Silurian Pinnacle Reefs, Michigan Basin, Search and Discovery Article \#50405 (2011) 
Vlastos, S., et al, 2003, Dual simulations of fluid flow and seismic wave propagation in a fractured network: Effects of changes in pore pressure on signature of seismic waves., SEG Technical Program Expanded Abstracts -- 2003 -- pp. 1382-1385

Vo-Thanh, D., 1990, Effects of fluid viscosity on shear-wave attenuation in saturated sandstones, Geophysics, vol. 55, no. 6, p. 712-722

Wang, Z., and Nur, A., 1990, Dispersion analysis of acoustic velocities in rocks: J. Acoust. Soc. Am., 87 , no. $6,2384-2395$.

Wang, Zhijing, 2001, Fundamentals of seismic rock physics, Geophysics , Vol. 66, No. 2

White, J. E., 1975, Seismic waves: Radiation, transmission and attenuation: New York, McGraw-Hill Book Co., Inc. - 1975, Computed seismic speeds and attenuation in rocks with partial gas saturation: Geophysics, v. 40, p. 224.

Winkler, K., and Nur, A., 1979, Pore fluids and seismic attenuation in rocks: Geophys. Res. Len., v. 6, p. 1.

Winkler, K., Nur, A., and Gladwin, M., 1979, Friction and seismic attenuation in rocks: Nature, v. 277, p. 528

Winkler, K.W., 1983, Frequency dependent ultrasonic properties of high-porosity sandstones, J. Geophys. Res. v. 88, No. B11, pp 9493-9499, 11/10/83 (ISSN01480227) Abstract only.

WORTHINGTON, M. H., 2007, The scaling of fracture compliance, Geological Society, London, Special Publications 2007; v. 270; p. 73-82

Wylie, Albert S. and Wood, James R., 2005, Well-log tomography and 3-D Imaging of Core and Log-Curve Amplitudes in a Niagraran Reef, Belle River Mills Field, St. Clair County, Michigan, United States. 
Wu, Xiaoyang, 2011, Frequency-dependent AVO attribute: theory and example, First Break volume 30, June 2012

Wyllie, M. R. J., 1962, Studies of Elastic Wave Attenuation in Porous Media, Geophysics, vol. XXVII, no. 5

Yamamoto, Tokuo, 2003, Imaging permeability structure within the highly permeable carbonate earth: Inverse theory and experiment, Geophysics, vol. 68, no. 4, p. 1189-1201

Yang, J., and Sato, T., 1998, Influence of Viscous Coupling on Seismic Reflection and Transmission in Saturated Porous Media, Bulletin of the Seismological Society of America, Vol. 88, No. 5, pp., October 1998

Zeng, H., 2007, Seismic imaging for seismic geomorphology beyond the seabed: potentials and challenges, Geological Society, London, Special Publications, 2007; 277: 15 - 28.

Zhang, Changjun, 2002, Estimation of Quality Factors - An Analytical Approach, CSEG Geophysics

Zhu, Yaping, 2007, Plane-wave attenuation anisotropy in orthorhombic media, Geophysics, Vol. 72, No. 1 